\title{
Automated Nanomole-Scale Reaction Screening Towards Benzoate Bioisosteres: A Photocatalyzed Approach to Highly Elaborated Bicyclo[1.1.1]Pentanes
}

James J. Mousseau ${ }^{1},{ }^{*}$ Matthew A. Perry ${ }^{1}$, Mark W. Bundesmann1, Gary M. Chinigo1, Chulho Choi ${ }^{1}$, Gary Gallego ${ }^{2}$, Robert W. Hicklin ${ }^{1}$, Susan Hoy ${ }^{1}$, David C. Limburg ${ }^{1}$, Neal W. Sach², Yuan Zhang ${ }^{3}$

${ }_{1}$ Pfizer Medicine Design, 445 Eastern Point Rd, Groton, CT 06340. ${ }^{2}$ Pfizer La Jolla Laboratories, 10770 Science Center Drive, San Diego, CA 92121. ${ }^{3}$ Pfizer Medicine Design, 610 Main St., Cambridge, MA 02139.

E-Mail: james.mousseau@gmail.com

Table of Contents

General Experimental Conditions 


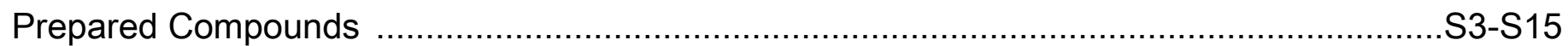

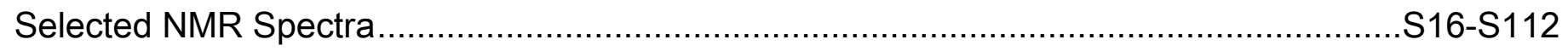

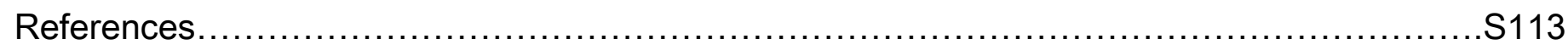


General Considerations: All reactions were run under an inert atmosphere (argon) with flame-dried glassware using standard techniques for manipulating air-sensitive compounds. Anhydrous solvents were obtained by filtration through drying columns (benzene) or by distillation over sodium and calcium hydride ( $p$-xylene, pyridine).

Flash column chromatography was performed using an Isco Isolera system using "Gold" columns with the indicated solvent system according to standard techniques. Reverse-phase column chromatography was performed using a Biotage Isolera Four using $30 \mathrm{~g}$ Biotage SNAP columns (5$95 \%$ water $\left(0.1 \% \mathrm{NH}_{4} \mathrm{OH}\right) / \mathrm{ACN}\left(0.1 \% \mathrm{NH}_{4} \mathrm{OH}\right) ; 25$ minute gradient; $30 \mathrm{~mL} / \mathrm{min}$ flow rate). Analytical thin-layer chromatography (TLC) was performed on pre-coated, glass-backed silica gel plates. Visualization of the developed chromatogram was performed by UV absorbance ( $254 \mathrm{~nm}$ ) and/or aqueous potassium permanganate.

Nuclear magnetic resonance spectra were recorded either on $300 \mathrm{MHz}$ or $400 \mathrm{MHz}$ spectrometers. Chemical shifts for ${ }^{1} \mathrm{H}$ NMR spectra are recorded in parts per million from tetramethylsilane with the solvent resonance as the internal standard (chloroform, $\delta=7.27 \mathrm{ppm}$ ). Data were reported as follows: chemical shift, multiplicity $(s=$ singlet, $d=$ doublet, $t=$ triplet, $m=$ multiplet and $\mathrm{br}=$ broad), coupling constant in $\mathrm{Hz}$ and integration. Chemical shifts for ${ }^{13} \mathrm{C}$ NMR spectra were recorded in parts per million from tetramethylsilane using the central peak of deuterochloroform (77.36 ppm) as the internal standard. Preparative High-Performance Liquid Chromatography was performed using reverse phase elution on a system equipped with simultaneous diode array UV detection. Data are reported as follows: (column type, eluent, flow rate: retention time $\left.\left(t_{r}\right)\right)$. Low resolution mass spectra were performed on a Waters Acquity UPLC equipped mass detector with simultaneous diode array UV and ESD detection.

Reagents: Commercial reagents were used as supplied or purified by standard techniques where necessary. Solvents were degassed with argon prior to use. Non-commercial compounds were obtained from Pfizer's proprietary compound library. Redox active esters used to prepare compound $6 a^{1}, 6 d^{2}, 6 g^{3}, 6 h^{4}$ have been described in the literature. 


\section{Experimental Procedures and Characterization Data}

\section{Synthesis of Redox Active Esters:}
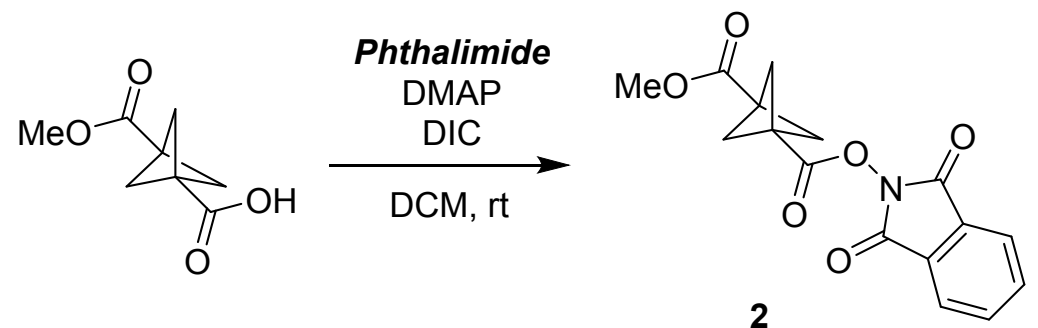

\section{1-(1,3-Dioxoisoindolin-2-yl) 3-methyl bicyclo[1.1.1]pentane-1,3-dicarboxylate (2).}

To an oven-dried round bottomed flask with stir bar was added 3(methoxycarbonyl)bicyclo[1.1.1]pentane-1-carboxylic acid $(4.0 \mathrm{~g}, 23.5 \mathrm{mmol}, 1$ equiv), $\mathrm{N}, \mathrm{N}-$ dimethylaminopyridine $(0.287 \mathrm{~g}, 2.35 \mathrm{mmol}, 10 \mathrm{~mol} \%), N, N$-diisopropylcarbodiimide (3.26 g, 25.9 mmol, 1.1 equiv), and and dichloromethane $(235 \mathrm{ml}, 0.1 \mathrm{M})$. To this solution was added $\mathrm{N}$ hydrophthalimide ( $4.22 \mathrm{~g}, 25.9 \mathrm{mmol}, 1.1$ equiv) and the reaction mixture was left to stir at rt for 16 $\mathrm{h}$. After $16 \mathrm{~h}$ the reaction was concentrated under reduced pressure and purified by automated flash chromatography $(0-100 \%$ EtOAc in DCM) and the product was identified by LCMS. Product was isolated as a white solid $(6.5 \mathrm{~g}, 88 \%) .{ }^{1} \mathrm{H} \mathrm{NMR}\left(\mathrm{CDCl}_{3}, 400 \mathrm{MHz}\right) \delta 7.91-7.89(\mathrm{~m}, 2 \mathrm{H}), 7.81-7.79$ $(\mathrm{m}, 2 \mathrm{H}), 3.73(\mathrm{~s}, 3 \mathrm{H}), 2.56(\mathrm{~s}, 6 \mathrm{H}) ;{ }^{13} \mathrm{C} \mathrm{NMR}\left(\mathrm{CDCl}_{3}, 100 \mathrm{MHz}\right) \delta 169.9,165.8,162.9,134.8,128.9$, 124.6, 54.5, 52.7, 38.6, 35.4; HRMS Calcd for $\mathrm{C}_{16} \mathrm{H}_{13} \mathrm{NO}_{6}(\mathrm{M}+\mathrm{H})+: 316.0816$. Found: 316.0814.

\section{General procedure Minisci protocol}

To a $20 \mathrm{~mL}$ vial equipped with a stir bar was added RAE 1-(1,3-dioxoisoindolin-2-yl) 3-methyl bicyclo[1.1.1]pentane-1,3-dicarboxylate 2 ( $0.25 \mathrm{mmol}, 1$ equiv), heterocycle ( $0.50 \mathrm{mmol}, 2$ equiv), and 4-DPAIPN $(0.006 \mathrm{mmol}, 2.5 \mathrm{~mol} \%)$. The vial was sealed, sparged $3 \mathrm{x}$ with argon after which either degassed MeCN or DMSO were added via syringe $(2.54 \mathrm{ml}, 0.1 \mathrm{M})$ followed by TFA $(0.5 \mathrm{mmol}$, 2 equiv). The reaction was stirred in a Heptochem reactor with a $405 \mathrm{nM}$ lamp for $16 \mathrm{~h}$. Following this time, the reaction was diluted with $\mathrm{DCM}$, washed with saturated aqueous $\mathrm{NaHCO}_{3}$ and back extracted with DCM. The combined organic extracts were dried over $\mathrm{Na}_{2} \mathrm{SO}_{4}$, concentrated under reduced pressure, and purified by automated flash chromatography. 

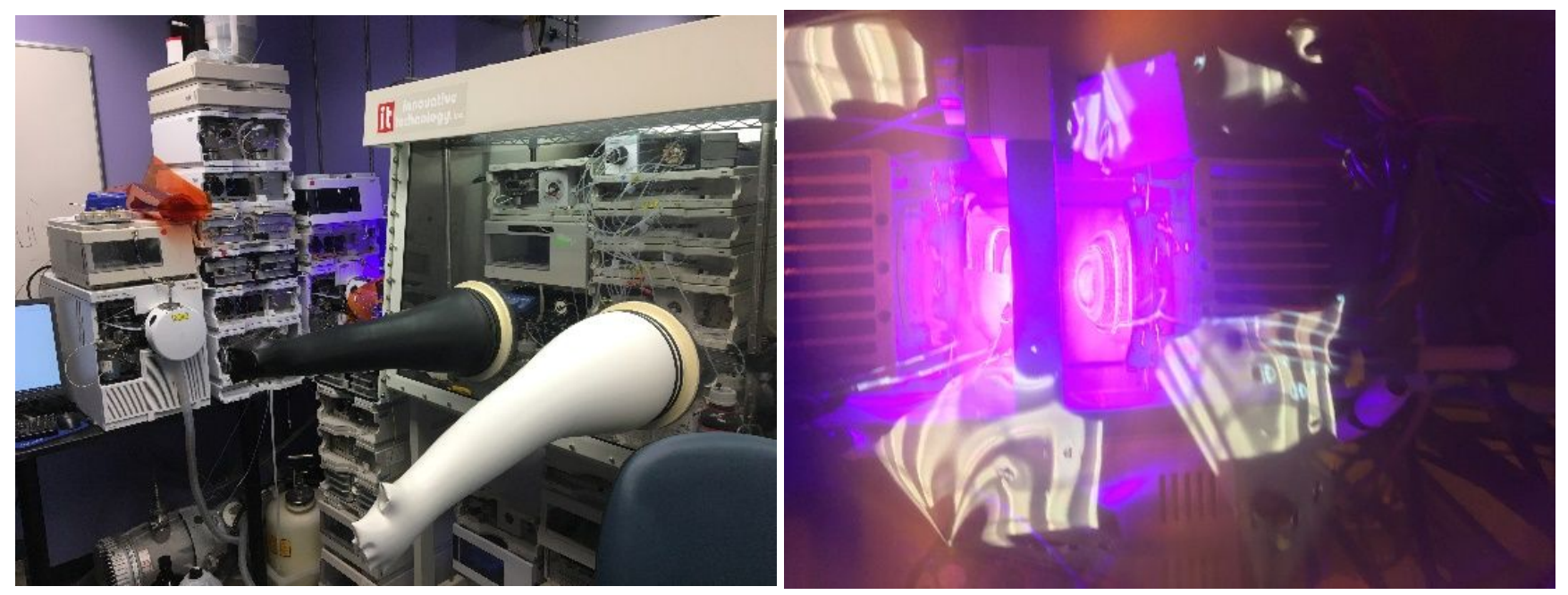

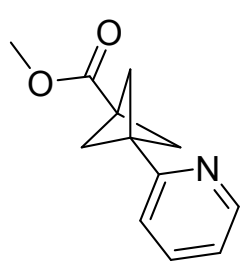

$4 a$

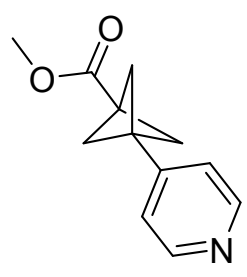

4b

Methyl 3-(pyridin-2-yl)bicyclo[1.1.1]pentane-1-carboxylate (4a) and methyl 3-(pyridin-4-yl)bicyclo[1.1.1]pentane-1-carboxylate (4b). The title compounds were prepared according to the general procedure using $\mathrm{MeCN}$ as the solvent and purified by automated flash chromatography $(0-50 \%$ EtOAc in DCM) and the products were identified by LCMS. Product $4 \mathrm{a}$ was isolated as a white amorphous solid ( $26 \mathrm{mg}, 50 \%$ ) and product $4 \mathrm{~b}$ as a white amorphous solid (12 mg, 23\%). The combined yield was $73 \%$. For compound 4a: ${ }^{1} \mathrm{H}$ NMR $\left(\mathrm{CDCl}_{3}, 400 \mathrm{MHz}\right) \delta 8.61-8.49(\mathrm{~m}, 1 \mathrm{H}), 7.64(\mathrm{td}, J=7.7,1.8 \mathrm{~Hz}, 1 \mathrm{H}), 7.23-7.08(\mathrm{~m}$, 2H), 3.72 (s, 3H), 2.42 (s, 6H).; ${ }^{13} \mathrm{C}$ NMR $\left(\mathrm{CDCl}_{3}, 100 \mathrm{MHz}\right) \delta 170.7,158.4,149.4,137.0,124.3$, 120.7, 53.2, 51.7, 43.0, 37.1; HRMS Calcd for $\mathrm{C}_{12} \mathrm{H}_{13} \mathrm{NO}_{2}[\mathrm{M}+\mathrm{H}]^{+}:$204.1019. Found: 204.1014. For compound 4a: ${ }^{1} \mathrm{H}$ NMR $\left(\mathrm{CDCl}_{3}, 400 \mathrm{MHz}\right) \delta 8.60(\mathrm{~d}, J=5 \mathrm{~Hz}, 2 \mathrm{H}), 7.21(\mathrm{~d}, J=5 \mathrm{~Hz}, 2 \mathrm{H}), 3.76(\mathrm{~s}$,

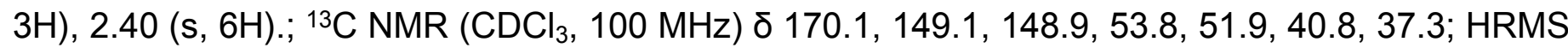
Calcd for $\mathrm{C}_{12} \mathrm{H}_{13} \mathrm{NO}_{2}[\mathrm{M}+\mathrm{H}]^{+}:$204.1019. Found: 204.1007.

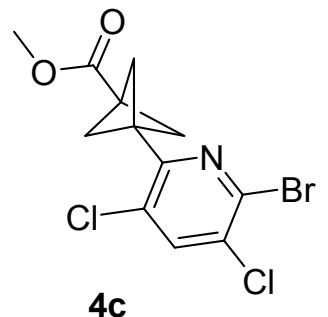

4c

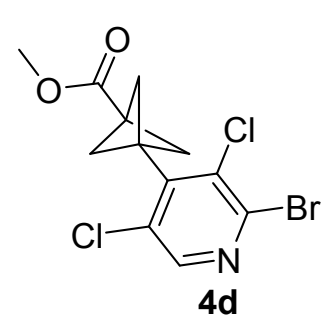

$4 d$
Methyl

yl)bicyclo[1.1.1]pentane-1-carboxylate 4c and methyl 3-(2bromo-3,5-dichloropyridin-4-yl)bicyclo[1.1.1]pentane-1-

carboxylate (4d). The title compounds were prepared according to the general procedure using $\mathrm{MeCN}$ as the solvent

and purified by automated flash chromatography (0-100\% EtOAc in heptane) and the products were identified by LCMS. Product $4 \mathrm{c}$ was isolated as a white amorphous solid (46 mg, $52 \%$ ) and product $4 \mathrm{~d}$ as a white amorphous solid $(21 \mathrm{mg}, 24 \%)$. The combined yield was $76 \%$. For compound $4 \mathrm{c}$ : ${ }^{1} \mathrm{H}$ 
$\operatorname{NMR}\left(\mathrm{CDCl}_{3}, 400 \mathrm{MHz}\right) \delta 7.67(\mathrm{~s}, 1 \mathrm{H}), 3.77$ (s, 3H), $2.51(\mathrm{~s}, 6 \mathrm{H}) . ;{ }^{13} \mathrm{C}$ NMR $\left(\mathrm{CDCl}_{3}, 100 \mathrm{MHz}\right) \delta$ 170.1, 153.2, 138.7, 138.6, 132.1, 130.7, 53.6, 51.2, 41.9, 39.2; HRMS Calcd for $\mathrm{C}_{12} \mathrm{H}_{10} \mathrm{BrCl}_{2} \mathrm{NO}_{2}(\mathrm{M}$ $+\mathrm{H})+$ : 349.9345. Found: 349.9358. For compound 4d: ${ }^{1} \mathrm{H}$ NMR $\left(\mathrm{CDCl}_{3}, 400 \mathrm{MHz}\right) \delta 8.20(\mathrm{~s}, 1 \mathrm{H})$, $3.73(\mathrm{~s}, 3 \mathrm{H}), 2.81(\mathrm{~s}, 6 \mathrm{H}) ;{ }^{13} \mathrm{C} \mathrm{NMR}\left(\mathrm{CDCl}_{3}, 100 \mathrm{MHz}\right) \delta$ 169.3, 147.6, 143.5, 142.2, 134.1, 131.9, 56.7, 51.9, 42.2, 40.0; HRMS Calcd for $\mathrm{C}_{12} \mathrm{H}_{10} \mathrm{BrCl}_{2} \mathrm{NO}_{2}[\mathrm{M}+\mathrm{H}]^{+}:$349.9345. Found: 349.9361.

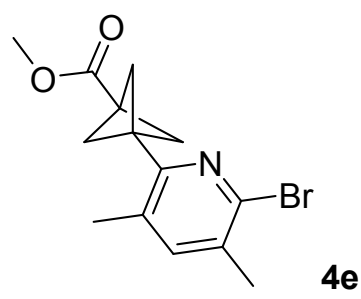

Methyl

3-(6-bromo-3,5-dimethylpyridin-2-yl)bicyclo[1.1.1]pentane-1carboxylate (4e). The title compound was prepared according to the general procedure using DMSO as the solvent and purified by automated flash chromatography (0-100\% EtOAc in heptane) and the product was identified by LCMS. Product $4 \mathrm{e}$ was isolated as a white amorphous solid $(48 \mathrm{mg}, 61 \%){ }^{1} \mathrm{H}$ $\operatorname{NMR}\left(\mathrm{CDCl}_{3}, 400 \mathrm{MHz}\right) \delta 7.91(\mathrm{~s}, 1 \mathrm{H}), 3.72(\mathrm{~s}, 3 \mathrm{H}), 2.68(\mathrm{~s}, 6 \mathrm{H}), 2.51(\mathrm{~s}, 3 \mathrm{H}), 2.34(\mathrm{~s}, 3 \mathrm{H}) . ;{ }^{13} \mathrm{C}$ $\operatorname{NMR}\left(\mathrm{CDCl}_{3}, 100 \mathrm{MHz}\right) \delta$ 169.5, 149.2, 145.5, 150.0, 133.0, 131.9, 55.8, 51.8, 42.9, 39.9, 20.0, 18.3; HRMS Calcd for $\mathrm{C}_{14} \mathrm{H}_{16} \mathrm{BrNO}_{2}[\mathrm{M}+\mathrm{H}]^{+}$310.0437. Found: 310.0430 .

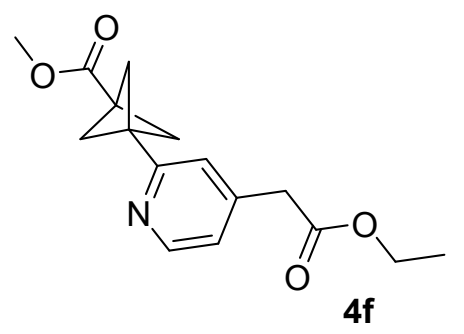

Methyl 3-(4-(2-ethoxy-2-oxoethyl)pyridin-2-yl)bicyclo[1.1.1]pentane-1carboxylate (4f). The title compound was prepared according to the general procedure using DMSO as the solvent and purified by automated flash chromatography (0-100\% EtOAc in heptane) and the product was identified by LCMS. Product $4 \mathrm{f}$ was isolated as a white amorphous solid (46 mg, 63\%) ${ }^{1} \mathrm{H} \mathrm{NMR}\left(\mathrm{CDCl}_{3}, 400 \mathrm{MHz}\right) \delta 8.51(\mathrm{~d}, J=6.0 \mathrm{~Hz}, 1 \mathrm{H}), 7.12-7.10(\mathrm{~m}, 2 \mathrm{H}), 4.19(\mathrm{q}, J=$ $7.3 \mathrm{~Hz}, 2 \mathrm{H}), 3.72(\mathrm{~s}, 3 \mathrm{H}), 3.60(\mathrm{~s}, 2 \mathrm{H}), 2.41(\mathrm{~s}, 6 \mathrm{H}), 1.27(\mathrm{t}, J=7.3 \mathrm{~Hz}, 3 \mathrm{H}) ;{ }^{13} \mathrm{C} \mathrm{NMR}\left(\mathrm{CDCl}_{3}, 100\right.$ $\mathrm{MHz}) \delta 170.6,170.0,159.3,149.5,143.4,122.9,121.6,61.3,53.2,51.7,42.4,40.7,37.2,14.1$; HRMS Calcd for $\mathrm{C}_{16} \mathrm{H}_{19} \mathrm{NO}_{4}[\mathrm{M}+\mathrm{H}]^{+}$290.1387. Found: 290.1394 .

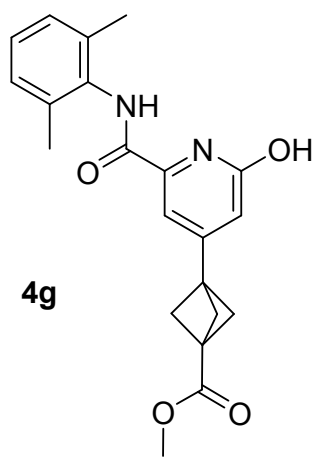

Methyl 3-(2-((2,6-dimethylphenyl)carbamoyl)-6-hydroxypyridin-4yl)bicyclo[1.1.1]pentane-1-carboxylate (4g). The title compound was prepared according to the general procedure using $\mathrm{MeCN}$ as the solvent and purified by automated flash chromatography (0-100\% EtOAc in DCM) and the product was identified by LCMS. Product $4 \mathrm{~g}$ was isolated as a yellow glass (35 $\mathrm{mg}, 38 \%$ ). $1 \mathrm{H}$ $\operatorname{NMR}\left(\mathrm{CDCl}_{3}, 400 \mathrm{MHz}\right) \delta 12.80(\mathrm{br} \mathrm{s}, 1 \mathrm{H}), 9.29(\mathrm{~s}, 1 \mathrm{H}), 7.40(\mathrm{~d}, J=7.0 \mathrm{~Hz}, 1 \mathrm{H})$, 
$7.31(\mathrm{~d}, J=7.4 \mathrm{~Hz}, 1 \mathrm{H}), 7.12-7.19(\mathrm{~m}, 3 \mathrm{H}), 3.67(\mathrm{~s}, 3 \mathrm{H}), 2.30(\mathrm{~s}, 6 \mathrm{H}), 2.12 \mathrm{ppm}(\mathrm{s}, 6 \mathrm{H}) \mathrm{ppm} ;{ }^{13} \mathrm{C}$ $\operatorname{NMR}\left(101 \mathrm{MHz}, \mathrm{CDCl}_{3}\right) \delta$ 169.7, 163.2, 158.4, 138.5, 135.6, 133.2, 128.4, 127.8, 110.5, 77.2, 77.0, 52.5, 51.5, 39.3, 38.2, 18.3 ppm; HRMS Calcd for $\mathrm{C}_{21} \mathrm{H}_{22} \mathrm{~N}_{2} \mathrm{O}_{4}[\mathrm{M}+\mathrm{H}]^{+} 367.1653$, found 367.1659.

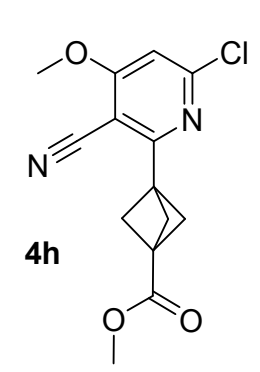

Methyl 3-(6-chloro-3-cyano-4-methoxypyridin-2-yl)bicyclo[1.1.1]pentane-1carboxylate (4h). The title compound was prepared according to the general procedure using $\mathrm{MeCN}$ as the solvent and purified by automated flash chromatography (0-50\% EtOAc in DCM) and the product was identified by LCMS. Product $4 \mathrm{~h}$ was isolated as a yellow oil $(20 \mathrm{mg}, 27 \%)$. $1 \mathrm{H}$ NMR (DMSO- $d_{6}, 500 \mathrm{MHz}$, TFA salt) $\delta 7.43(\mathrm{~s}, 1 \mathrm{H}), 4.02(\mathrm{~s}, 3 \mathrm{H}), 3.65(\mathrm{~s}, 3 \mathrm{H}), 2.47$ (s, 6H) ppm; ${ }^{13} \mathrm{C}$ NMR (126 $\mathrm{MHz}$, DMSO- $d_{6}$, TFA salt) $\delta 169.6,169.4,161.8,155.3,113.9,107.7,96.7,58.4,53.6,52.1,42.0$, 37.7 ppm; HRMS Calcd for $\mathrm{C}_{14} \mathrm{H}_{13} \mathrm{CIN}_{2} \mathrm{O}_{3}[\mathrm{M}+\mathrm{H}]^{+} 293.0688$, found 293.0693 .

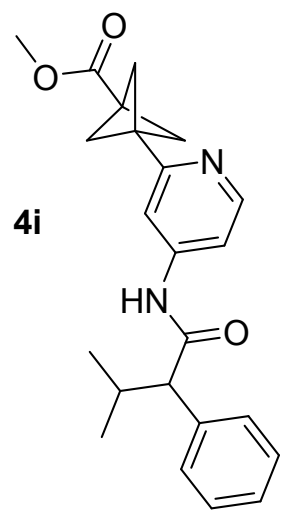

Methyl 3-(4-(3-methyl-2-phenylbutanamido)pyridin-2-yl)bicyclo[1.1.1]pentane-1carboxylate (4i). The title compound was prepared according to the general procedure using $\mathrm{MeCN}$ as the solvent and purified by automated flash chromatography (0-50\% EtOAc in DCM) and the product was identified by LCMS. Product 4i was isolated as a clear oil $(42 \mathrm{mg}, 44 \%)$. $1 \mathrm{H} \mathrm{NMR}\left(\mathrm{CDCl}_{3}, 400 \mathrm{MHz}\right) \delta$ $8.55(\mathrm{~s}, 1 \mathrm{H}), 8.31(\mathrm{~d}, J=5.7 \mathrm{~Hz}, 1 \mathrm{H}), 7.57(\mathrm{~d}, J=2.2 \mathrm{~Hz}, 1 \mathrm{H}), 7.46-7.06(\mathrm{~m}, 6 \mathrm{H})$, $3.71(\mathrm{~s}, 3 \mathrm{H}), 3.16(\mathrm{~d}, J=10.1 \mathrm{~Hz}, 1 \mathrm{H}), 2.51(\mathrm{dp}, J=10.1,6.6 \mathrm{~Hz}, 1 \mathrm{H}), 2.37(\mathrm{~s}, 6 \mathrm{H})$, $1.12(\mathrm{~d}, J=6.4 \mathrm{~Hz}, 3 \mathrm{H}), 0.74 \mathrm{ppm}(\mathrm{d}, J=6.7 \mathrm{~Hz}, 3 \mathrm{H}) \mathrm{ppm} ;{ }^{13} \mathrm{C} \mathrm{NMR}(101 \mathrm{MHz}$, $\left.\mathrm{CDCl}_{3}\right) \delta 173.7,171.1,159.6,149.8,147.2,138.9,129.4,129.0,128.2,112.8,111.4,63.3,53.8$, 52.5, 42.8, 37.8, 32.3, 22.4, 20.9 ppm; HRMS Calcd for $\mathrm{C}_{23} \mathrm{H}_{27} \mathrm{~N}_{2} \mathrm{O}_{3}[\mathrm{M}+\mathrm{H}]^{+}$379.2016, found 379.2023.

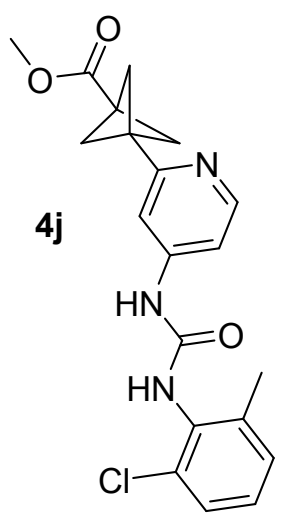

Methyl 3-(4-(3-(2-chloro-6-methylphenyl)ureido)pyridin-2-yl)bicyclo[1.1.1]pentane1-carboxylate (4j). The title compound was prepared according to the general procedure using $\mathrm{MeCN}$ as the solvent and purified by Biotage reverse phase preparative chromatography (0-50\% water/ACN, 20 min gradient) and the product was identified by LCMS. Product $4 \mathrm{j}$ was isolated as a yellow amorphous solid ( 23 mg, 23\%). 1H NMR ( $\left.\mathrm{CDCl}_{3}, 400 \mathrm{MHz}\right) \delta 11.39(\mathrm{~s}, 1 \mathrm{H}), 8.88(\mathrm{~s}, 1 \mathrm{H}), 8.02$ (d, $J=7.0$ $\mathrm{Hz}, 1 \mathrm{H}$ ), 7.96 (br s, 1H), 7.66 (br s, $1 \mathrm{H}), 7.20$ (dd, $J=2.3,7.0 \mathrm{~Hz}, 1 \mathrm{H}), 7.08-7.14$ 
(m, 2H), $3.70(\mathrm{~s}, 3 \mathrm{H}), 2.46(\mathrm{~s}, 6 \mathrm{H}), 2.29(\mathrm{~s}, 3 \mathrm{H}) \mathrm{ppm} .{ }^{13} \mathrm{C} \mathrm{NMR}\left(101 \mathrm{MHz}, \mathrm{CDCl}_{3}\right) \delta$ 170.6, 158.9, 153.3, 149.1, 147.6, 136.6, 132.5, 132.2, 129.3, 128.2, 127.3, 111.6, 110.1, 53.1, 51.8, 42.2, 37.1, 18.8 ppm. HRMS Calcd for $\mathrm{C}_{20} \mathrm{H}_{20} \mathrm{CIN}_{3} \mathrm{O}_{3}[\mathrm{M}+\mathrm{H}]^{+} 386.1265$, found 386.1266.

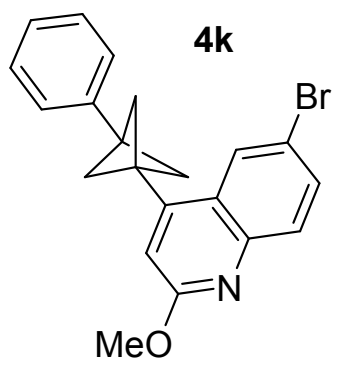

6-bromo-2-methoxy-4-(3-phenylbicyclo[1.1.1]pentan-1-yl)quinoline (4k). The title compounds was prepared according to the general procedure using $\mathrm{MeCN}$ as the solvent and purified by automated reverse phase HPLC (Chiracel OJ-3 $4.6 \times 100 \mathrm{~mm}$, 3um column; 5-60\% $\mathrm{MeOH} / 10 \mathrm{mM} \mathrm{NH}_{3}$ in $\mathrm{CO}_{2}, 0-3$ minutes, 4 $\mathrm{mL} /$ minute flow rate) and the products were identified by LCMS. Product $4 \mathrm{k}$ was isolated as a clear oil (17 mg, 20\%). ${ }^{1} \mathrm{H}$ NMR (DMSO- $d_{6}, 400 \mathrm{MHz}$ ) for 4substituted product 4k $\delta 1 \mathrm{H}$ NMR $(400 \mathrm{MHz}$, Chloroform- $d) \delta 8.19(\mathrm{~d}, J=2.1 \mathrm{~Hz}, 1 \mathrm{H}), 7.73(\mathrm{~d}, J=8.8$ $\mathrm{Hz}, 1 \mathrm{H}), 7.67$ (dd, J= 8.9, $2.2 \mathrm{~Hz}, 1 \mathrm{H}), 6.68(\mathrm{~s}, 1 \mathrm{H}), 4.04(\mathrm{~s}, 3 \mathrm{H}), 3.75(\mathrm{~s}, 3 \mathrm{H}), 2.60(\mathrm{~s}, 6 \mathrm{H}) .{ }^{13} \mathrm{C}$ NMR (101 MHz, CDCl3) $\delta 170.0,162.5,146.5,146.0,132.6,129.9,127.0,125.8,117.4,113.0,54.5,53.6$, 52.0, 41.5, 38.6; HRMS Calcd for $\mathrm{C}_{17} \mathrm{H}_{17} \mathrm{BrNO}_{3}[\mathrm{M}+\mathrm{H}]^{+}$362.0386. Found: 362.0404 .

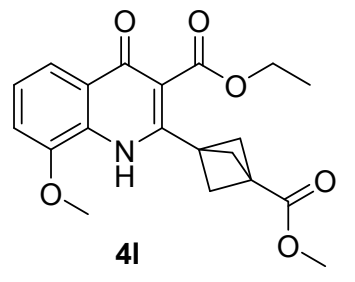

Ethyl 8-methoxy-2-(3-(methoxycarbonyl)bicyclo[1.1.1]pentan-1-yl)-4-oxo-1,4dihydroquinoline-3-carboxylate (4I). The title compound was prepared according to the general procedure using $\mathrm{MeCN}$ as the solvent and purified by automated flash chromatography (0-50\% EtOAc in DCM) and the product was identified by LCMS. Product $4 \mathrm{I}$ was isolated as a clear oil $(51 \mathrm{mg}, 54 \%)$. $1 \mathrm{H} \mathrm{NMR}\left(\mathrm{CDCl}_{3}, 400\right.$ $\mathrm{MHz}) \delta 8.29$ (br s, 1H), 7.87 (d, J=8.2 Hz, 1H), 7.25 (t, J= $7.9 \mathrm{~Hz}, 1 \mathrm{H}), 7.06$ (dd, J= 8.0, $1.0 \mathrm{~Hz}$, $1 \mathrm{H}), 4.42(\mathrm{q}, J=7.2 \mathrm{~Hz}, 2 \mathrm{H}), 4.03(\mathrm{~s}, 3 \mathrm{H}), 3.75(\mathrm{~s}, 3 \mathrm{H}), 2.52(\mathrm{~s}, 6 \mathrm{H}), 1.42 \mathrm{ppm}(\mathrm{t}, J=7.2 \mathrm{~Hz}, 3 \mathrm{H})$ ppm; ${ }^{13} \mathrm{C}$ NMR $\left(101 \mathrm{MHz}, \mathrm{CDCl}_{3}\right) \delta 175.1,169.0,166.6,147.2,144.1,129.3,125.7,123.7,117.5$, 116.6, 111.0, 61.5, 56.1, 53.3, 51.9, 39.0, 37.4, 14.2 ppm; HRMS Calcd for $\mathrm{C}_{20} \mathrm{H}_{21} \mathrm{NO}_{6}[\mathrm{M}+\mathrm{H}]^{+}$ 372.1442 , found 372.1456 .

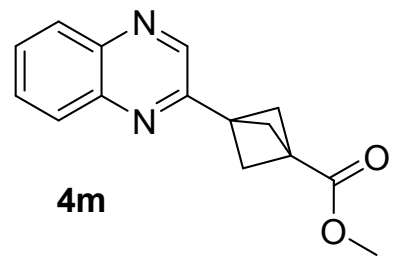

Methyl 3-(quinoxalin-2-yl)bicyclo[1.1.1]pentane-1-carboxylate (4m). The title compound was prepared according to the general procedure using $\mathrm{MeCN}$ as the solvent and purified by automated flash chromatography (0-50\% EtOAc in DCM) and the product was identified by LCMS. Product $4 \mathrm{~m}$ was isolated as a tan solid (20 mg, 31\%). $1 \mathrm{H} \mathrm{NMR}\left(\mathrm{CDCl}_{3}, 500 \mathrm{MHz}\right) \delta 8.80(\mathrm{~s}, 1 \mathrm{H}), 8.03-8.15(\mathrm{~m}, 2 \mathrm{H}), 7.69-7.84$ (m, 2H), $3.76(\mathrm{~s}, 3 \mathrm{H}), 2.59$ (s, 6H) ppm; ${ }^{13} \mathrm{C}$ NMR $\left(126 \mathrm{MHz}, \mathrm{CDCl}_{3}\right) \delta$ 170.2, 153.2, 143.4, 142.0, 
S10

141.6, 130.2, 129.6, 129.3, 129.2, 53.5, 51.9, 41.4, 37.9 ppm; HRMS Calcd for $\mathrm{C}_{15} \mathrm{H}_{14} \mathrm{~N}_{2} \mathrm{O}_{2}[\mathrm{M}+\mathrm{H}]^{+}$ 255.1128 , found 255.1127 .

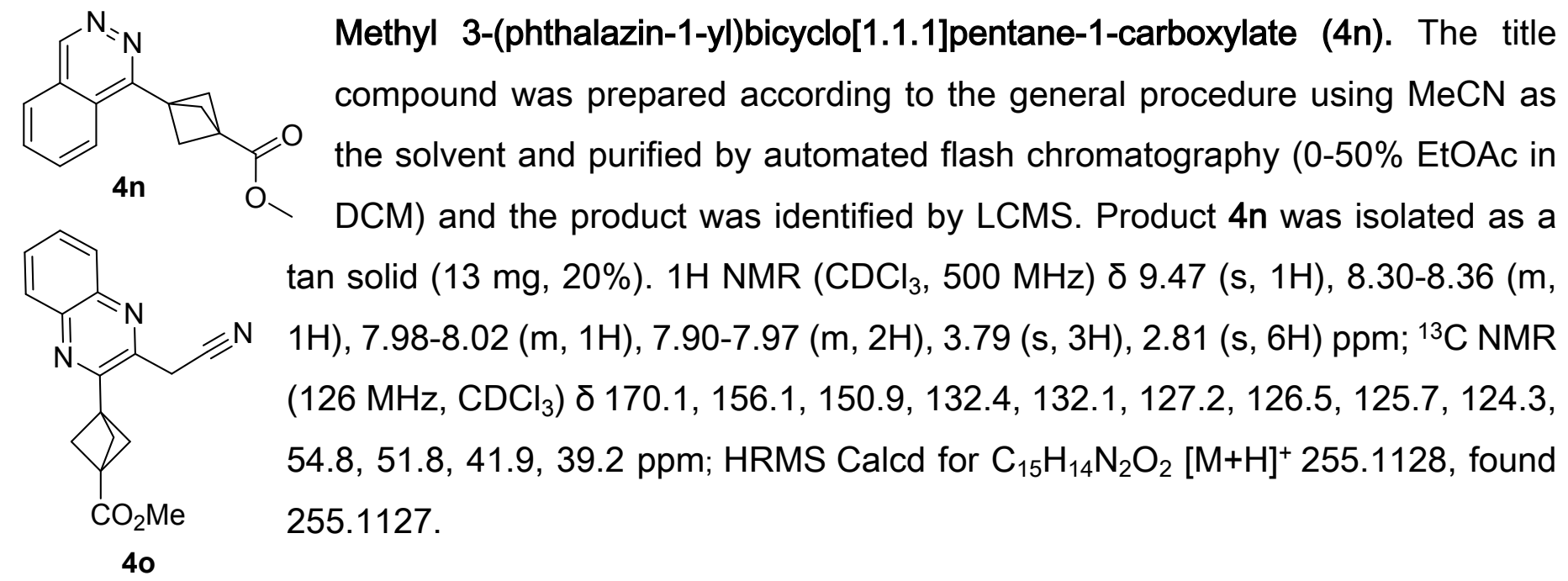

Methyl 3-(3-(cyanomethyl)quinoxalin-2-yl)bicyclo[1.1.1]pentane-1-carboxylate (40). The title compound was prepared according to the general procedure using DMSO as the solvent and purified by reverse phase chromatography (gradient of $0-100 \%$ water-MeCN, $0.1 \%$ formic acid modifier) and lyophilized to yield product 40 as an off white amorphous solid (43 mg, 60\%). ${ }^{1} \mathrm{H} \mathrm{NMR}(400 \mathrm{MHz}$, DMSO- $d_{6}$ ) $\delta$ 8.10-8.05 (m, 2H), 7.87-7.84 (sxt, J=3.12 Hz, 2H), 4.62 (s, 2H), 3.67 (s, 3H), 2.56 (s, $6 \mathrm{H}) ;{ }^{13} \mathrm{C}$ NMR $\left(126 \mathrm{MHz}\right.$, DMSO- $\left.d_{6}\right) \delta$ 169.4, 151.0, 146.7, 140.7, 140.5, 140.0, 130.4, 128.5, 128.3, 117.0, 53.1, 53.3, 53.0, 51.6, 25.9; HRMS Calcd for $\mathrm{C}_{17} \mathrm{H}_{15} \mathrm{~N}_{3} \mathrm{O}_{2}[\mathrm{M}+\mathrm{H}]^{+}$294.1237. Found: 294.1247.

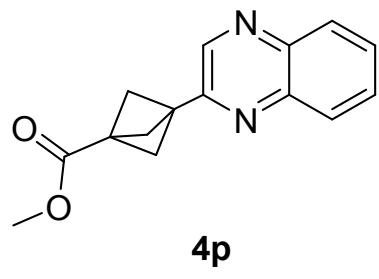

Methyl 3-(quinoxalin-2-yl)bicyclo[1.1.1]pentane-1-carboxylate (4p). The title compound was prepared according to the general procedure using DMSO as the solvent and purified by reverse phase chromatography (gradient of 0 $100 \%$ water-MeCN, $0.1 \%$ formic acid modifier) and lyophilized to yield product $4 \mathrm{p}$ as an off white amorphous solid (24 mg, 37\%). ${ }^{1} \mathrm{H}$ NMR (400 MHz, DMSO- $\left.d_{6}\right) \delta 8.88(\mathrm{~s}, 1 \mathrm{H})$, 8.08-8.06 (dq, $J=3.51,1.95,1.95,1.95,1.95,2 \mathrm{H}$ ), 7.86-7.78 (app. quind, $J=3.5,2.0 \mathrm{~Hz}, 2 \mathrm{H}$ ), 3.74 (s, 3H), 2.57 (s, 6H); ${ }^{13} \mathrm{C}$ NMR (126 MHz, DMSO-d 6 ) $\delta 170.4,154.0,153.5,143.4,141.6,141.1$, 130.4, 129.7, 129.6, 128.4, 52.9, 52.8, 51.0; HRMS Calcd for $\mathrm{C}_{15} \mathrm{H}_{14} \mathrm{~N}_{2} \mathrm{O}_{2}[\mathrm{M}+\mathrm{H}]^{+}$255.1128. Found: 255.1135 . 


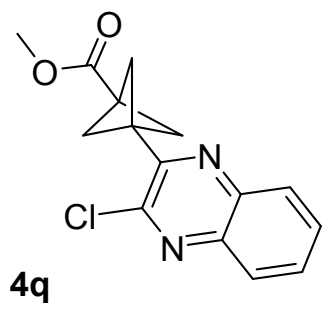

Methyl 3-(3-chloroquinoxalin-2-yl)bicyclo[1.1.1]pentane-1-carboxylate (4q). The title compound was prepared according to the general procedure using DMSO as the solvent and purified by automated flash chromatography (0-15\% EtOAc in

DCM) and the product was identified by LCMS. Product $4 q$ was isolated as an off-white solid (21 mg, 29\%). ${ }^{1} \mathrm{H}$ NMR (400 MHz, Methanol- $\left.d_{4}\right) \delta 8.19-8.08(\mathrm{~m}$, 1H), $8.05-7.96(\mathrm{~m}, 1 \mathrm{H}), 7.95-7.77(\mathrm{~m}, 2 \mathrm{H}), 3.77(\mathrm{~s}, 3 \mathrm{H}), 2.68(\mathrm{~s}, 6 \mathrm{H}) .{ }^{13} \mathrm{C}$ NMR $(126 \mathrm{MHz}, \mathrm{MeOD})$ $\delta 170.43,150.85,146.26,140.99,140.71,130.72,130.32$, 128.48, 127.51, 53.09, 50.94, 41.88, 38.28. HRMS Calcd for $\mathrm{C}_{15} \mathrm{H}_{14} \mathrm{CIN}_{2} \mathrm{O}_{2}[\mathrm{M}+\mathrm{H}]^{+}$289.0744. Found: 289.0733.

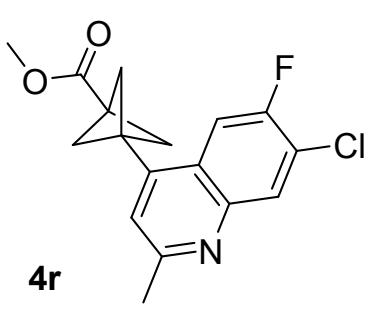

Methyl 3-(7-chloro-6-fluoro-2-methylquinolin-4-yl)bicyclo[1.1.1]pentane-1carboxylate (4r). The title compound was prepared according to the general procedure using DMSO as the solvent and purified by reverse phase chromatography (gradient of $0-100 \%$ water-MeCN, $0.1 \%$ formic acid modifier) and lyophilized to yield product $4 \mathrm{r}$ as an off white amorphous solid $(14 \mathrm{mg}$, 17\%). ${ }^{1} \mathrm{H}$ NMR (400 MHz, Methanol- $\left.d_{4}\right) \delta 8.19-7.98(\mathrm{~m}, 2 \mathrm{H}), 7.30(\mathrm{~s}, 1 \mathrm{H}), 3.77(\mathrm{~s}, 3 \mathrm{H}), 2.71$ (s, $3 \mathrm{H}), 2.66(\mathrm{~s}, 6 \mathrm{H}) ;{ }^{13} \mathrm{C}$ NMR $\left(126 \mathrm{MHz}\right.$, Methanol- $\left.d_{4}\right) \delta 170.1,159.8,156.1,154.1,144.9$ (d, $\mathrm{J}_{\mathrm{C}-\mathrm{F}}=75$ $\mathrm{Hz}), 129.6,124.8\left(\mathrm{~m} \mathrm{~d}, J_{\mathrm{C}-\mathrm{Cl}}=9 \mathrm{~Hz}\right), 124.1\left(\mathrm{~d}, J_{\mathrm{C}-\mathrm{F}}=21 \mathrm{~Hz}\right), 122.1,109.6\left(\mathrm{~d}, J_{\mathrm{C}-\mathrm{F}}=24 \mathrm{~Hz}\right), 53.8,51.0$, 40.9, 38.2, 23.2; HRMS Calcd for $\mathrm{C}_{17} \mathrm{H}_{15} \mathrm{FCINO}_{2}[\mathrm{M}+\mathrm{H}]^{+}$320.0854. Found: 320.0847 .

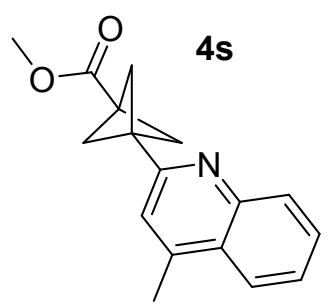

Methyl 3-(4-methylquinolin-2-yl)bicyclo[1.1.1]pentane-1-carboxylate (4s). The title compound was prepared according to the general procedure using $\mathrm{MeCN}$ as the solvent and purified by automated flash chromatography (0-20\% EtOAc in DCM) and the product was identified by LCMS. Product $4 s$ was isolated as a white amorphous solid (42 $\mathrm{mg}, 62 \%){ }^{1} \mathrm{H} \mathrm{NMR}\left(\mathrm{CDCl}_{3}, 400 \mathrm{MHz}\right) \delta 8.10(\mathrm{~d}, J=$ $8.4 \mathrm{~Hz}, 1 \mathrm{H}), 7.96(\mathrm{~d}, J=8.3 \mathrm{~Hz}, 1 \mathrm{H}), 7.69(\mathrm{t}, J=6.9 \mathrm{~Hz}, 1 \mathrm{H}), 7.52(\mathrm{t}, J=7.0 \mathrm{~Hz}, 1 \mathrm{H}), 7.18(\mathrm{~s}, 1 \mathrm{H})$, 3.75 (s, 3H), $2.69(\mathrm{~s}, 3 \mathrm{H}), 2.52(\mathrm{~s}, 6 \mathrm{H}) ;{ }^{13} \mathrm{C} \mathrm{NMR}\left(\mathrm{CDCl}_{3}, 100 \mathrm{MHz}\right) \delta 170.8,158.3,147.7,144.0$, 
S12

129.8, 129.3, 127.2, 126.0, 123.6, 119.4, 53.3, 51.8, 42.9, 37.3, 18.7 ; HRMS Calcd for $\mathrm{C}_{17} \mathrm{H}_{17} \mathrm{NO}_{2}$ $[\mathrm{M}+\mathrm{H}]^{+}$268.1327. Found: 268.1339 .

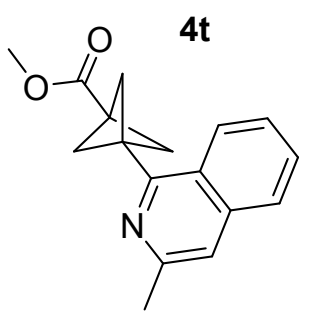

Methyl 3-(3-methylisoquinolin-1-yl)bicyclo[1.1.1]pentane-1-carboxylate (4t). The title compound was prepared according to the general procedure using $\mathrm{MeCN}$ as the solvent and purified by automated flash chromatography (0-10\% EtOAc in DCM) and the product was identified by LCMS. Product $4 \mathrm{~s}$ was isolated as a white amorphous solid (52 mg, 77\%) ${ }^{1} \mathrm{H}$ NMR $\left(\mathrm{CDCl}_{3}, 400 \mathrm{MHz}\right) \delta 8.33(\mathrm{~d}, J=8.2 \mathrm{~Hz}$, $1 \mathrm{H}), 7.74(\mathrm{~d}, J=8.4 \mathrm{~Hz}, 1 \mathrm{H}), 7.61(\mathrm{t}, J=6.9 \mathrm{~Hz}, 1 \mathrm{H}), 7.50(\mathrm{t}, J=7.1 \mathrm{~Hz}, 1 \mathrm{H}), 7.38(\mathrm{~s}, 1 \mathrm{H}), 3.76(\mathrm{~s}$, 3H), 2.71 (s, 6H), $2.67(\mathrm{~s}, 3 \mathrm{H}) ;{ }^{13} \mathrm{C} \mathrm{NMR}\left(\mathrm{CDCl}_{3}, 100 \mathrm{MHz}\right) \delta 170.8,156.5,150.7,129.6,126.9$, 125.9, 125.5, 125.4, 118.7, 54.9, 51.8, 43.6, 39.0, 24.4; HRMS Calcd for $\mathrm{C}_{17} \mathrm{H}_{17} \mathrm{NO}_{2}[\mathrm{M}+\mathrm{H}]^{+}$ 268.1338. Found: 268.1332.

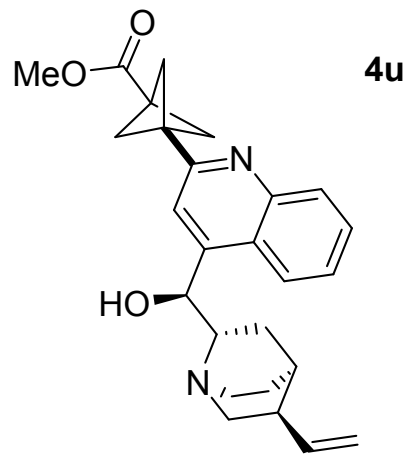

Methyl 3-(4-((S)-hydroxy((1S,2S,4S,5R)-5-vinylquinuclidin-2-yl)methyl) quinolin-2-yl)bicyclo[1.1.1]pentane-1-carboxylate (4u). The title compound was prepared according to the general procedure using $\mathrm{MeCN}$ as the solvent and purified by automated flash chromatography (0-10\% EtOAc in DCM) and the product was identified by LCMS. Product $4 u$ was isolated as a beige amorphous solid (44 mg, 41\%) ${ }^{1} \mathrm{H}$ NMR $\left(\mathrm{CDCl}_{3}, 400 \mathrm{MHz}\right) \delta 7.90$ (d, J=8.1 $\mathrm{Hz}, 1 \mathrm{H}), 7.69(\mathrm{~d}, J=8.3 \mathrm{~Hz}, 1 \mathrm{H}), 7.57(\mathrm{~s}, 1 \mathrm{H}), 7.48(\mathrm{t}, J=6.9 \mathrm{~Hz}, 1 \mathrm{H}), 7.21$ (t, J=7.3 Hz, 1H),6.24 (s, 1H), 5.57-5.49 (m, 1H), 5.04-4.98 (m, 2H), 4.41 (brt, 1H), $3.76(\mathrm{~s}, 1 \mathrm{H})$, 3.48-3.35 (m, 2H), 3.21-3.07 (m, 2H), 2.67 (bs, 1.5H), 2.53 (s, 6H), 2.23 (brt, 1H), 2.09-2.02 (m, 2H), 1.76 (brt, $1 \mathrm{H}), 1.34$ (brt, $1 \mathrm{H}) ;{ }^{13} \mathrm{C} \mathrm{NMR}\left(\mathrm{CDCl}_{3}, 100 \mathrm{MHz}\right) \delta$ 170.7, 158.2, 147.6, 145.5, 137.3, 130.0, 129.2, 126.8, 123.1, 121.5, 118.3, 116.0, 66.5, 60.6, 54.6, 53.4, 52.8, 51.8, 44.0, 43.1, 37.3, 26.8, 24.4, 18.3; HRMS Calcd for $\mathrm{C}_{26} \mathrm{H}_{30} \mathrm{~N}_{2} \mathrm{O}_{3}[\mathrm{M}+\mathrm{H}]^{+}$419.2329. Found: 419.2320.

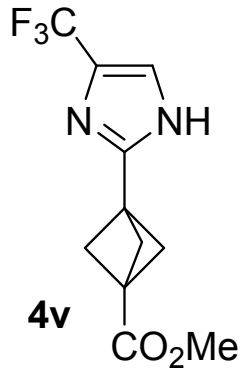

Methyl 3-(4-(trifluoromethyl)-1H-imidazol-2-yl)bicyclo[1.1.1]pentane-1-carboxylate $(4 \mathrm{v})$. The title compound was prepared according to the general procedure using $\mathrm{MeCN}$ as the solvent and purified by reverse phase chromatography (gradient of 0 $100 \%$ water-MeCN, $0.1 \%$ formic acid modifier) and lyophilized to yield product $4 \mathrm{v}$ as an off white amorphous solid (11 mg, 17\%) ${ }^{1} \mathrm{H}$ NMR $\left(400 \mathrm{MHz}\right.$, Methanol- $\left.d_{4}\right) \delta 7.50$ 
(s, 1H), $3.73(\mathrm{~s}, 3 \mathrm{H}), 2.46(\mathrm{~s}, 6 \mathrm{H}) .{ }^{13} \mathrm{C}$ NMR (126 MHz, MeOD) $\delta 169.94,147.75,133.9,121.8$ (q, $\left.J_{C-F}=266.0 \mathrm{~Hz}\right), 117.05,53.15,50.93,37.89,34.62$. HRMS Calcd for $\mathrm{C}_{11} \mathrm{H}_{12} \mathrm{~F}_{3} \mathrm{~N}_{2} \mathrm{O}_{2}[\mathrm{M}+\mathrm{H}]^{+}$ 261.0841. Found: 261.0841.

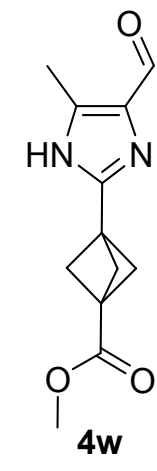

Methyl 3-(4-formyl-5-methyl-1H-imidazol-2-yl)bicyclo[1.1.1]pentane-1-carboxylate (4w). The title compound was prepared according to the general procedure using $\mathrm{MeCN}$ as the solvent and purified by automated flash chromatography (0-100\% EtOAc in DCM) and the product was identified by LCMS. Product $4 \mathrm{w}$ was isolated as a yellow oil $(25 \mathrm{mg}, 42 \%) .1 \mathrm{H}$ $\operatorname{NMR}\left(\mathrm{CDCl}_{3}, 400 \mathrm{MHz}\right) \delta 10.54(\mathrm{br} \mathrm{s}, 1 \mathrm{H}), 7.50(\mathrm{~s}, 1 \mathrm{H}), 3.73(\mathrm{~s}, 3 \mathrm{H}), 2.58(\mathrm{~s}, 3 \mathrm{H}), 2.55$ ppm (s, 6H) ppm; ${ }^{13} \mathrm{C}$ NMR $\left(126 \mathrm{MHz}, \mathrm{CDCl}_{3}\right) \delta 193.6,170.8,135.7,133.4,53.8,51.8$, 43.9, 38.2, 11.9 ppm; HRMS Calcd for $\mathrm{C}_{12} \mathrm{H}_{14} \mathrm{~N}_{2} \mathrm{O}_{3}[\mathrm{M}+\mathrm{H}]^{+}$235.1077, found 235.1076.

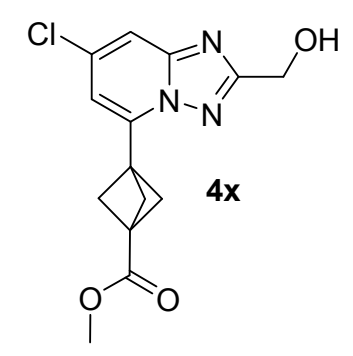

Methyl 3-(7-chloro-2-(hydroxymethyl)-[1,2,4]triazolo[1,5-a]pyridin-5yl)bicyclo[1.1.1]pentane-1-carboxylate (4x). The title compound was prepared according to the general procedure using $\mathrm{MeCN}$ as the solvent and purified by automated flash chromatography (0-100\% EtOAc in DCM) and the products were identified by LCMS. Product $4 \mathrm{x}$ was isolated as a clear oil $(22 \mathrm{mg}, 28 \%, 1: 1.3$ mixture of regioisomers). $1 \mathrm{H} \mathrm{NMR}\left(\mathrm{CDCl}_{3}, 400 \mathrm{MHz}\right.$, minor isomer denoted by $\left.{ }^{*}\right) \delta 8.32(\mathrm{~d}, J=7.4$ $\mathrm{Hz}, 1 \mathrm{H}), 7.62(\mathrm{~d}, J=2.3 \mathrm{~Hz}, 1 \mathrm{H})^{*}, 6.92(\mathrm{~d}, J=7.0 \mathrm{~Hz}, 1 \mathrm{H}), 6.74(\mathrm{~d}, J=2.0 \mathrm{~Hz}, 1 \mathrm{H})^{*}, 4.93(\mathrm{~s}, 2 \mathrm{H})^{*}$, $4.92(\mathrm{~s}, 2 \mathrm{H}), 3.74(\mathrm{~s}, 3 \mathrm{H})^{*}, 3.72(\mathrm{~s}, 3 \mathrm{H}), 2.79(\mathrm{~s}, 9 \mathrm{H}), 2.65 \mathrm{ppm}(\mathrm{s}, 6 \mathrm{H})^{*} \mathrm{ppm} ;{ }^{13} \mathrm{C} \mathrm{NMR}(101 \mathrm{MHz}$, $\left.\mathrm{CDCl}_{3}\right) \delta 169.9,169.5,166.6,166.5,151.7,151.5,139.3,136.3,134.3,126.6,124.2,116.3,113.8$, 113.2, 58.8, 58.6, 55.2, 52.9, 51.9, 51.7, 42.0, 40.1, 39.3, 38.2 ppm; HRMS Calcd for $\mathrm{C}_{14} \mathrm{H}_{14} \mathrm{CIN}_{3} \mathrm{O}_{3}$ $[\mathrm{M}+\mathrm{H}]^{+} 308.0797$, found 308.0796 .

Methyl 3-(7-chloroimidazo[1,2-a]pyridin-5-yl)bicyclo[1.1.1]pentane-1-carboxylate

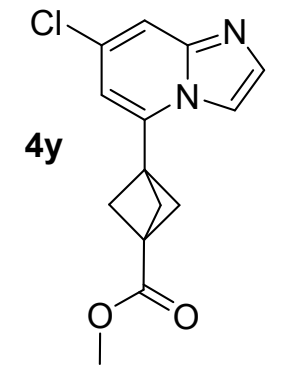

(4y). The title compound was prepared according to the general procedure using $\mathrm{MeCN}$ as the solvent and purified by automated flash chromatography $(0-100 \%$ EtOAc in DCM) and the product was identified by LCMS. Product $4 y$ was isolated as 
a yellow oil (18 mg, 26\%). 1H NMR (DMSO- $d_{6}, 500 \mathrm{MHz}$, TFA salt) $\delta 8.43$ (d, $\left.J=1.5 \mathrm{~Hz}, 1 \mathrm{H}\right), 8.17$ (d, $J=2.0 \mathrm{~Hz}, 1 \mathrm{H}$ ), $8.06(\mathrm{~d}, J=1.5 \mathrm{~Hz}, 1 \mathrm{H}), 7.26(\mathrm{~d}, J=2.0 \mathrm{~Hz}, 1 \mathrm{H}), 3.69(\mathrm{~s}, 3 \mathrm{H}), 2.66(\mathrm{~s}, 6 \mathrm{H}) \mathrm{ppm}$; ${ }^{13} \mathrm{C}$ NMR $\left(126 \mathrm{MHz}\right.$, DMSO- $d_{6}$, TFA salt) $\delta 168.8,141.4,138.0,136.0,127.0,115.7,113.4,111.9$, 52.1, 51.7, 40.4, 38.2 ppm; HRMS Calcd for $\mathrm{C}_{14} \mathrm{H}_{13} \mathrm{CIN}_{2} \mathrm{O}_{2}[\mathrm{M}+\mathrm{H}]^{+} 277.0739$, found 277.0743.
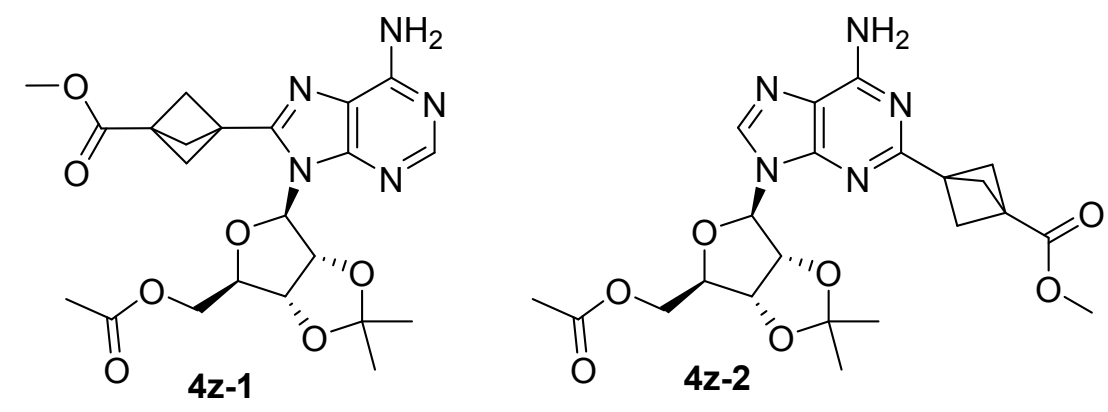
Methyl 3-(9-((3aR,4R,6R,6aR)-6- (acetoxymethyl)-2,2-
dimethyltetrahydrofuro[3,4- d][1,3]dioxol-4-yl)-6-amino-9H-purin- 8-yl)bicyclo[1.1.1]pentane-1- carboxylate (4z-1) and Methyl 3-(9((3aR,4R,6R,6aR)-6-(acetoxymethyl)-2,2-dimethyltetrahydrofuro[3,4-d][1,3]dioxol-4-yl)-6-amino-9Hpurin-2-yl)bicyclo[1.1.1]pentane-1-carboxylate (4z-2). The title compounds were prepared according to the general procedure using MeCN as the solvent and purified by RPLC $(0-100 \%$ water/ACN $0.1 \%$ formic acid, $30 \mathrm{~g}$ SNAP C18 column) and the products were identified by LCMS. Product $4 \mathrm{z}$ was isolated as a white solid (30 mg, 25\%, 1.5:1 ratio). Further purification by reverse phase column chromatography (5-95\% water $\left(0.1 \% \mathrm{NH}_{4} \mathrm{OH}\right) / \mathrm{ACN}\left(0.1 \% \mathrm{NH}_{4} \mathrm{OH}\right) ; 25$ minute gradient; $30 \mathrm{~mL} / \mathrm{min}$ flow rate) provided the individual isomers as white solids. $1 \mathrm{H}$ NMR of $4 \mathrm{z}-1$ (DMSO- $d_{6}, 500 \mathrm{MHz}$ ) $\delta 8.13(\mathrm{~s}, 1 \mathrm{H}), 7.35$ (br s, 2H), 6.12 (d, $J=2.0 \mathrm{~Hz}, 1 \mathrm{H}$ ), 5.80 (dd, J=6.2, 2.1 $\mathrm{Hz}, 1 \mathrm{H}), 5.12(\mathrm{br} \mathrm{dd}, J=6.4,3.2 \mathrm{~Hz}, 1 \mathrm{H}), 4.31-4.39(\mathrm{~m}, 1 \mathrm{H}), 4.19(\mathrm{br} \mathrm{dd}, J=11.6,5.0 \mathrm{~Hz}, 1 \mathrm{H})$, 4.04 (br dd, $J=11.6,7.0 \mathrm{~Hz}, 1 \mathrm{H}), 3.66(\mathrm{~s}, 3 \mathrm{H}), 2.53-2.58(\mathrm{~m}, 5 \mathrm{H}), 1.94(\mathrm{~s}, 3 \mathrm{H}), 1.56(\mathrm{~s}, 3 \mathrm{H}), 1.35$ ppm (s, 3H) ppm; ${ }^{13} \mathrm{C}$ NMR of 4z-1 (126 MHz, DMSO- $\left.d_{6}\right) \delta 170.4,169.3,156.2,153.0,150.1$, 148.0, 118.4, 114.0, 90.0, 84.6, 82.6, 82.0, 63.8, 54.5, 53.2, 52.1, 36.4, 27.6, 25.8, 20.9 ppm; $1 \mathrm{H}$ NMR of 4z-2 (DMSO- $\left.d_{6}, 500 \mathrm{MHz}\right) \delta 8.26(\mathrm{~s}, 1 \mathrm{H}), 7.37(\mathrm{~s}, 2 \mathrm{H}), 6.25(\mathrm{~d}, J=1.5 \mathrm{~Hz}, 1 \mathrm{H}), 5.41(\mathrm{dd}, J$ $=6.1,1.5 \mathrm{~Hz}, 1 \mathrm{H}), 5.15(\mathrm{dd}, J=6.1,3.4 \mathrm{~Hz}, 1 \mathrm{H}), 4.31-4.39(\mathrm{~m}, 1 \mathrm{H}), 4.22-4.29(\mathrm{~m}, 1 \mathrm{H}), 4.14-4.22$ (m, 1H), $3.65(\mathrm{~s}, 3 \mathrm{H}), 2.31(\mathrm{~s}, 6 \mathrm{H}), 1.97(\mathrm{~s}, 3 \mathrm{H}), 1.55(\mathrm{~s}, 3 \mathrm{H}), 1.35(\mathrm{~s}, 3 \mathrm{H}) \mathrm{ppm} ;{ }^{13} \mathrm{C}$ NMR of 4z-2 $\left(126 \mathrm{MHz}\right.$, DMSO- $\left.d_{6}\right) \delta 170.5,170.4,160.8,156.5,149.3,140.8,118.5,113.7,89.4,85.2,84.2$, 82.1, 64.6, 53.2, 51.9, 43.1, 37.1, 27.4, 25.6, 21.0 ppm; HRMS Calcd for $\mathrm{C}_{22} \mathrm{H}_{27} \mathrm{~N}_{5} \mathrm{O}_{7}[\mathrm{M}+\mathrm{H}]^{+}$ 474.1983, found 474.1995 .

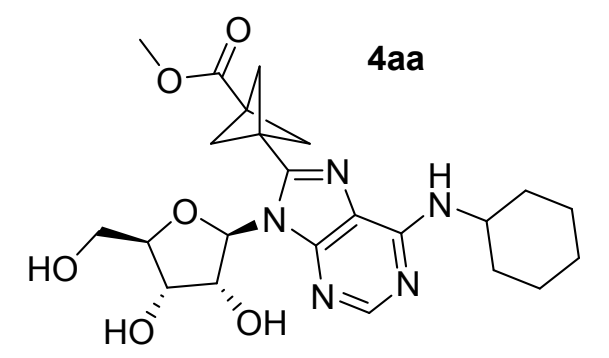

Methyl 3-(6-(cyclohexylamino)-9-(3,4-dihydroxy-5(hydroxymethyl)tetrahydrofuran-2-yl)-9H-purin-8- 
yl)bicyclo[1.1.1]pentane-1-carboxylate (4aa). The title compound was prepared according to the general procedure using $\mathrm{MeCN}$ as the solvent and purified by Biotage reverse phase preparative chromatography (0-50\% water/ACN, 20 min gradient) and the product was identified by LCMS. Product 4aa was isolated as a yellow amorphous solid (24 mg, 20\%). $1 \mathrm{H} \mathrm{NMR}\left(\mathrm{CDCl}_{3}, 400 \mathrm{MHz}\right) \delta$ $7.67(\mathrm{~s}, 1 \mathrm{H}), 6.07$ (br s, 1H), $5.76(\mathrm{~d}, J=7.3 \mathrm{~Hz}, 2 \mathrm{H}), 5.04(\mathrm{~m}, 1 \mathrm{H}), 5.46(\mathrm{~d}, J=4.9 \mathrm{~Hz}, 1 \mathrm{H}), 4.34(\mathrm{~s}$, $1 \mathrm{H}), 4.13(\mathrm{br} \mathrm{s}, 1 \mathrm{H}), 3.92(\mathrm{~d}, J=12.7 \mathrm{~Hz}, 1 \mathrm{H}), 3.71-3.74(\mathrm{~m}, 4 \mathrm{H}), 2.61(\mathrm{~s}, 2 \mathrm{H}), 2.41(\mathrm{~s}, 6 \mathrm{H}), 2.06-$ $2.11(\mathrm{~m}, 2 \mathrm{H}), 1.76-1.81(\mathrm{~m}, 2 \mathrm{H}), 1.68-1.69(\mathrm{~m}, 1 \mathrm{H}), 1.40-1.49(\mathrm{~m}, 2 \mathrm{H}), 1.25-1.35 \mathrm{ppm}(\mathrm{m}, 3 \mathrm{H}) .{ }^{13} \mathrm{C}$ NMR $\left(101 \mathrm{MHz}, \mathrm{CDCl}_{3}\right)$ 170.9, 155.5, 148.1, 139.6, 130.1, 124.7, 91.3, 87.7, 73.7, 72.9, 63.1, 53.4, 51.6, 42.5, 41.0, 37.0, 33.0, 32.9, 25.6, 24.8, 24.7 ppm. HRMS Calcd for $\mathrm{C}_{23} \mathrm{H}_{31} \mathrm{~N}_{5} \mathrm{O}_{6}[\mathrm{M}+\mathrm{H}]^{+}$ 474.2347, found 474.2345 .

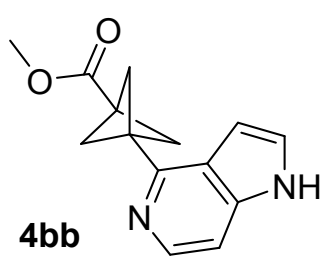

Methyl 3-(1H-pyrrolo[3,2-c]pyridin-4-yl)bicyclo[1.1.1]pentane-1-carboxylate (4bb). The title compound was prepared according to the general procedure using $\mathrm{MeCN}$ as the solvent and purified by Biotage reverse phase preparative chromatography $(0-50 \%$ water/ACN, 20 min gradient) and the product was identified by LCMS. Product 4bb was isolated as a purple amorphous solid (10 mg, 16\%). 1H NMR $\left(\mathrm{CDCl}_{3}, 400 \mathrm{MHz}\right) \delta 9.61(\mathrm{br} \mathrm{s}, 1 \mathrm{H}), 8.23(\mathrm{~d}, J=5.9 \mathrm{~Hz}, 1 \mathrm{H}), 7.31(\mathrm{~d}, J=3.1 \mathrm{~Hz}, 1 \mathrm{H}), 7.29(\mathrm{~d}, J=6.2$ $\mathrm{Hz}, 1 \mathrm{H}), 6.76(\mathrm{~d}, J=3.1 \mathrm{~Hz}, 1 \mathrm{H}), 3.74(\mathrm{~s}, 3 \mathrm{H}), 2.64 \mathrm{ppm}(\mathrm{s}, 6 \mathrm{H}) \cdot{ }^{13} \mathrm{C} \mathrm{NMR}\left(101 \mathrm{MHz}, \mathrm{CDCl}_{3}\right) \delta=$ $170.5,150.6,140.4,139.1,125.8,122.6,106.0,101.7,54.0,51.8,42.1$, 38.3 ppm. HRMS Calcd for $\mathrm{C}_{14} \mathrm{H}_{14} \mathrm{~N}_{2} \mathrm{O}_{2}[\mathrm{M}+\mathrm{H}]^{+} 243.1128$, found 243.1124 .

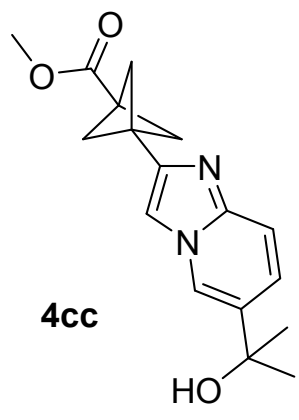

Methyl 3-(6-(2-hydroxypropan-2-yl)imidazo[1,2-a]pyridin-2yl)bicyclo[1.1.1]pentane-1-carboxylate (4cc). The title compound was prepared according to the general procedure using $\mathrm{MeCN}$ as the solvent and purified by Biotage reverse phase preparative chromatography $(0-50 \%$ water/ACN, $20 \mathrm{~min}$ gradient) and the product was identified by LCMS. Product 4cc was isolated as a yellow amorphous solid (31 mg, 41\%). 1H NMR (CDCl3, $400 \mathrm{MHz}) \delta 8.19$ (d, J = $2.0 \mathrm{~Hz}, 1 \mathrm{H}), 8.04(\mathrm{~d}, \mathrm{~J}=9.4 \mathrm{~Hz}, 1 \mathrm{H}), 7.80(\mathrm{~d}, \mathrm{~J}=2.0 \mathrm{~Hz}, 1 \mathrm{H}), 7.72(\mathrm{~d}, \mathrm{~J}=9.8 \mathrm{~Hz}, 1 \mathrm{H}), 3.74(\mathrm{~s}, 3 \mathrm{H})$, 2.94 (s, 6H), 1.72 ppm (s, 6H). 13C NMR $\delta(101 \mathrm{MHz}, \mathrm{CDCl} 3): \delta$ 169.2, 140.5, 139.2, 136.3, 132.3, 
123.0, 114.0, 111.5, 72.8, 56.1, 51.9, 41.0, 33.1 ppm. HRMS Calcd for $\mathrm{C}_{17} \mathrm{H}_{20} \mathrm{~N}_{2} \mathrm{O}_{3}[\mathrm{M}+\mathrm{H}]^{+} 301.1547$. Found: 301.1547.

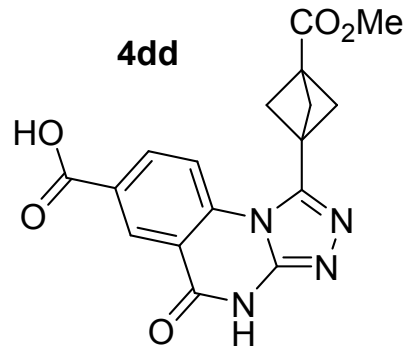

\section{1-(3-(methoxycarbonyl)bicyclo[1.1.1]pentan-1-yl)-5-oxo-4,5-dihydro-} $[1,2,4]$ triazolo[4,3-a]quinazoline-7-carboxylic acid (4dd). The title compounds were prepared according to the general procedure using $\mathrm{MeCN}$ as the solvent and purified by automated reverse phase HPLC (5-95\% water (1\% TFA)/ACN gradient, Sunfire C18 19x100mm 5um column). Product 4dd was isolated as a white amorphous solid (9 mg, 10\%) ${ }^{1} \mathrm{H}$ NMR (DMSO- $\left.d_{6}, 400 \mathrm{MHz}\right) \delta 13.41(\mathrm{~s}, 1 \mathrm{H}$ ), $13.03(\mathrm{~s}, 1 \mathrm{H}), 8.69$ (d, J = 2.1 Hz, 1H), $8.54(\mathrm{dd}, \mathrm{J}=8.7,2.2 \mathrm{~Hz}, 1 \mathrm{H}), 8.12(\mathrm{~d}, \mathrm{~J}=8.7 \mathrm{~Hz}, 1 \mathrm{H}), 3.69$ (s, 3H), 2.70 (s, 6H). ${ }^{13} \mathrm{C}$ NMR (101 MHz, DMSO- $\left.\alpha_{6}\right) \delta$ 169.49, 166.30, 160.50, 149.05, 145.81, 136.97, 136.24, 130.37, 129.05, 118.32, 118.22, 53.72, 52.15, 40.91, 35.49; HRMS Calcd for $\mathrm{C}_{17} \mathrm{H}_{14} \mathrm{~N}_{4} \mathrm{O}_{5}[\mathrm{M}+\mathrm{H}]^{+}$355.1025. Found: 355.1037.

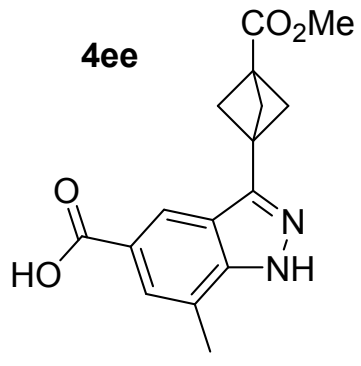

\section{3-(3-(methoxycarbonyl)bicyclo[1.1.1]pentan-1-yl)-7-methyl-1H-indazole-5-} carboxylic acid (4ee). The title compound was prepared according to the general procedure using $\mathrm{MeCN}$ as the solvent and purified by automated reverse phase HPLC (5-95\% water (1\% TFA)/ACN gradient, Sunfire C18 $19 \times 100 \mathrm{~mm} 5$ um column). Product 4ee was isolated as a white amorphous solid (7 mg, 10\%) ${ }^{1} \mathrm{H}$ NMR (DMSO- $\left.d_{6}, 400 \mathrm{MHz}\right) \delta 13.37$ (s, 1H), 12.88 (s, 1H), 8.36 (s, 1H), $7.21(\mathrm{~d}, \mathrm{~J}=1.1 \mathrm{~Hz}, 1 \mathrm{H}), 3.66(\mathrm{~s}, 3 \mathrm{H}), 2.57(\mathrm{~s}, 6 \mathrm{H}), 2.49(\mathrm{~d}, \mathrm{~J}=1.0 \mathrm{~Hz}, 3 \mathrm{H}) .{ }^{13} \mathrm{C}$ NMR $(101$ $\mathrm{MHz}$, DMSO) $\delta 171.10,170.06,134.50,128.28,126.38,126.11,122.39,54.89,51.93,40.91,38.38$, 16.82. HRMS Calcd for $\mathrm{C}_{16} \mathrm{H}_{16} \mathrm{~N}_{2} \mathrm{O}_{4}[\mathrm{M}+\mathrm{H}]^{+}$301.1183. Found: 301.1181.

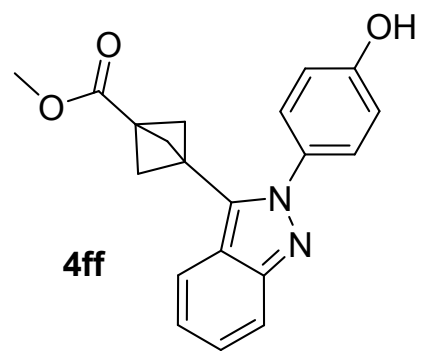

Methyl 3-(2-(4-hydroxyphenyl)-2H-indazol-3-yl)bicyclo[1.1.1]pentane-1carboxylate (4ff). The title compound was prepared according to the general procedure using $\mathrm{MeCN}$ as the solvent and purified by automated reverse phase HPLC (5-95\% water (1\% TFA)/ACN gradient, Sunfire C18 $19 \times 100 \mathrm{~mm} 5 \mathrm{um}$ column). Product $4 \mathrm{ff}$ was isolated as a white amorphous solid (36 mg, 42\%). ${ }^{1} \mathrm{H}$ NMR (DMSO- $d_{6}, 400 \mathrm{MHz}$ ) $\delta 10.04$ (s, 1H), 7.79 
(dt, J = 8.6, 1.1 Hz, 1H), $7.60(\mathrm{dt}, \mathrm{J}=8.7,1.0 \mathrm{~Hz}, 1 \mathrm{H}), 7.33-7.26(\mathrm{~m}, 3 \mathrm{H}), 7.08$ (ddd, J = 8.6, 6.6, $0.9 \mathrm{~Hz}, 1 \mathrm{H}), 6.96-6.90(\mathrm{~m}, 2 \mathrm{H}), 3.59(\mathrm{~s}, 3 \mathrm{H}), 2.29(\mathrm{~s}, 6 \mathrm{H}) ;{ }^{13} \mathrm{C}$ NMR $\left(100 \mathrm{MHz}, \mathrm{DMSO}-d_{6}\right) \delta 169.4$, 158.8, 147.8, 133.7, 131.7, 129.0, 126.6, 121.8, 121.4, 120.9, 117.6, 115.6, 54.6, 52.0, 40.9, 35.0; HRMS Calcd for $\mathrm{C}_{20} \mathrm{H}_{18} \mathrm{~N}_{2} \mathrm{O}_{3}[\mathrm{M}+\mathrm{H}]^{+}$335.1390. Found: 335.1391.

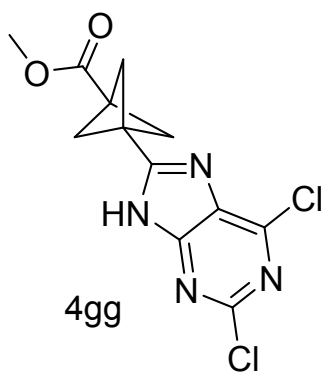

Methyl 3-(2,6-dichloro-9H-purin-8-yl)bicyclo[1.1.1]pentane-1-carboxylate (4gg). The title compound was prepared according to the general procedure using $\mathrm{MeCN}$ as the solvent and purified by automated flash chromatography $(0-100 \%$ EtOAc in DCM) and the product was identified by LCMS. Product $4 \mathrm{gg}$ was isolated as a yellow solid (24 mg, 30\%) ${ }^{1} \mathrm{H}$ NMR (400 MHz, DMSO- $\left.d_{6}\right) \delta 3.66$ (s, $3 \mathrm{H}), 3.32(\mathrm{~s}, 6 \mathrm{H}) ;{ }^{13} \mathrm{C}$ NMR $\left(100 \mathrm{MHz}, \mathrm{DMSO}-d_{6}\right) \delta$ 168.9, 150.6, 53.3, 51.6, 38.1, 35.50; HRMS Calcd for $\mathrm{C}_{12} \mathrm{H}_{10} \mathrm{Cl}_{2} \mathrm{~N}_{4} \mathrm{O}_{2}[\mathrm{M}+\mathrm{H}]^{+} 313.0254$, found: 313.0266 .

Note: purines such as $\mathbf{4 g g}$ are known to exhibit tautomerism that can lead to missing quaternary carbons in ${ }^{13} \mathrm{C}$ NMR. Hocek, M.; Pohl, R. Synthesis, 2004, 17, 2869-2876. 10.1055/s-2004-834881

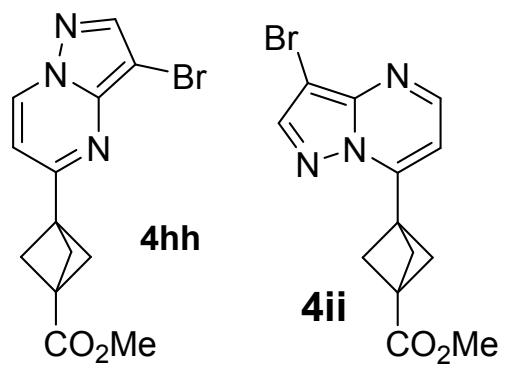

Methyl 3-(3-bromopyrazolo[1,5-a]pyrimidin-5-yl)bicyclo[1.1.1]pentane1-carboxylate (4hh) and Methyl 3-(3-bromopyrazolo[1,5-a]pyrimidin-7yl)bicyclo[1.1.1]pentane-1-carboxylate (4ii). The title compounds were prepared according to the general procedure using $\mathrm{MeCN}$ as the solvent. A mixture of indicated isomers was formed in a $0.7: 1$ ratio (4hh : 4ii). The mixture was purified by reverse phase chromatography (gradient of $0-100 \%$ water-MeCN, $0.1 \%$ formic acid modifier) and lyophilized to yield product $4 \mathrm{hh}$ as an off white amorphous solid (6 mg, $8 \%$ ) and product 4 ii as an off white amorphous solid (8 $\mathrm{mg}$, 10\%). For 4hh: ${ }^{1} \mathrm{H}$ NMR (400 MHz, Methanol- $\left.d_{4}\right) \delta 8.70$ (d, $\left.J=7.2 \mathrm{~Hz}, 1 \mathrm{H}\right), 8.03(\mathrm{~s}, 1 \mathrm{H}), 6.90$ (d, J $=7.2 \mathrm{~Hz}, 1 \mathrm{H}), 3.62(\mathrm{~s}, 3 \mathrm{H}), 2.37$ (s, 6H). ${ }^{13} \mathrm{C}$ NMR (101 MHz, MeOD) $\delta 170.44,159.98,144.80$, 144.71, 135.89, 106.73, 83.03, 52.78, 50.94, 41.91, 37.02. HRMS Calcd for $\mathrm{C}_{13} \mathrm{H}_{13} \mathrm{BrN}_{3} \mathrm{O}_{2}[\mathrm{M}+\mathrm{H}]^{+}$ 322.0191. Found: 322.0188. For 4ii: ${ }^{1} \mathrm{H}$ NMR $\left(400 \mathrm{MHz}\right.$, Methanol- $\left.d_{4}\right) \delta 8.41(\mathrm{~d}, J=4.2 \mathrm{~Hz}, 1 \mathrm{H})$, $8.10(\mathrm{~s}, 1 \mathrm{H}), 6.77$ (d, J=4.2 Hz, 1H), $3.63(\mathrm{~s}, 3 \mathrm{H}), 2.57$ (s, 6H). ${ }^{13} \mathrm{C}$ NMR (101 MHz, MeOD) $\delta 169.77$, $149.98,146.01,145.20,144.24,106.66,83.58,52.54,51.01,39.34,37.64$. HRMS Calcd for $\mathrm{C}_{13} \mathrm{H}_{13} \mathrm{BrN}_{3} \mathrm{O}_{2}[\mathrm{M}+\mathrm{H}]^{+}$322.0191. Found: 322.0186 . 
S18

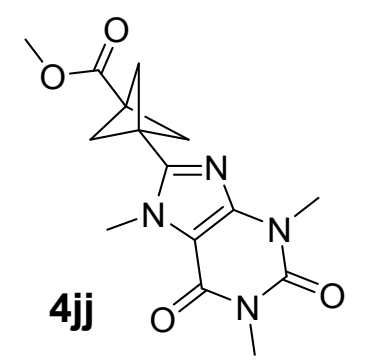

Methyl

3-(1,3,7-trimethyl-2,6-dioxo-2,3,6,7-tetrahydro-1H-purin-8yl)bicyclo[1.1.1]pentane-1-carboxylate (4jj). The title compound was prepared according to the general procedure using $\mathrm{MeCN}$ as the solvent and purified by automated flash chromatography (0-50\% EtOAc in DCM) and the product was identified by LCMS. Product $4 \mathrm{jj}$ was isolated as a off white amorphous solid (52 mg, 64\%) ${ }^{1} \mathrm{H} \mathrm{NMR}\left(\mathrm{CDCl}_{3}, 400 \mathrm{MHz}\right) \delta 3.99$ (s, 3H), 3.73 (s, 3H), 3.55 (s, 3H), 3.39 (s, 3H), 2.58 (s, $6 \mathrm{H}) ;{ }^{13} \mathrm{C} \mathrm{NMR}\left(\mathrm{CDCl}_{3}, 100 \mathrm{MHz}\right) \delta 169.3,155.4,151.7,149.7,147.7,17.8,54.1,52.0,39.6,35.5$, 32.5, 29.8, 27.9 ; HRMS Calcd for $\mathrm{C}_{15} \mathrm{H}_{18} \mathrm{~N}_{4} \mathrm{O}_{4}[\mathrm{M}+\mathrm{H}]^{+}$319.1401. Found: 319.1398.

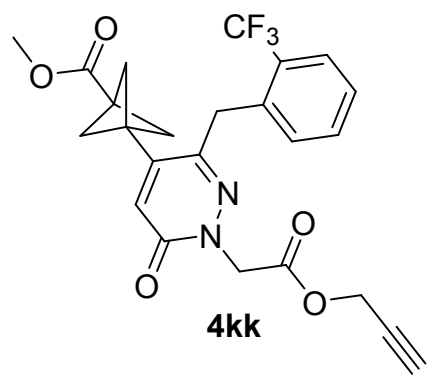

Methyl

3-(6-oxo-1-(2-oxo-2-(prop-2-yn-1-yloxy)ethyl)-3-(2(trifluoromethyl)benzyl)-1,6-dihydropyridazin-4-yl)bicyclo[1.1.1]pentane-1carboxylate (4kk). The title compound was prepared according to the general procedure using DMSO as the solvent and purified by automated flash chromatography (0-10\% EtOAc in DCM) and the product was identified by LCMS. Product 4kk was isolated as a off white amorphous solid (33 mg, 26\%) ${ }^{1} \mathrm{H}$ NMR $\left(\mathrm{CDCl}_{3}, 400 \mathrm{MHz}\right) \delta 7.70(\mathrm{~d}, J=7.6 \mathrm{~Hz}, 1 \mathrm{H}), 7.51(\mathrm{t}, J=7.9 \mathrm{~Hz}, 1 \mathrm{H})$, $7.38(\mathrm{t}, J=7.6 \mathrm{~Hz}, 1 \mathrm{H}), 7.30(\mathrm{~d}, J=7.3 \mathrm{~Hz}, 1 \mathrm{H}), 6.75(\mathrm{~s}, 1 \mathrm{H}), 4.89(\mathrm{~s}, 2 \mathrm{H}), 4.80(\mathrm{~d}, J=2.5 \mathrm{~Hz}, 2 \mathrm{H})$, $4.08(\mathrm{~s}, 2 \mathrm{H}), 3.68(\mathrm{~s}, 3 \mathrm{H}), 2.52(\mathrm{t}, J=2.4 \mathrm{~Hz}, 1 \mathrm{H}), 2.36(\mathrm{~s}, 6 \mathrm{H}) ;{ }^{13} \mathrm{C} \mathrm{NMR}\left(\mathrm{CDCl}_{3}, 100 \mathrm{MHz}\right) \delta 170.0$, $166.9,159.5,145.9,140.2,135.6,135.6,132.2,131.4,129.2,128.9,128.6,127.2,126.3,126.3$, 126.2, 126.1, 125.7, 123.2, 76.9, 75.6, 53.0, 52.9, 52.7, 51.7, 39.2, 38.5, 36.9, 36.9, 36.9; ${ }^{19} \mathrm{~F}$ NMR $\left(376 \mathrm{MHz}, \mathrm{CDCl}_{3}\right) \delta$-59.42; HRMS Calcd for $\mathrm{C}_{24} \mathrm{H}_{21} \mathrm{~F}_{3} \mathrm{~N}_{2} \mathrm{O}_{5}[\mathrm{M}+\mathrm{H}]^{+}$475.1476. Found: 475.1486.

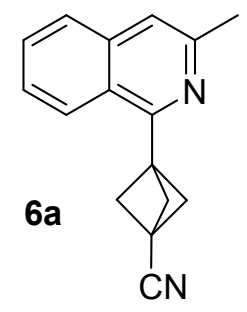

3-(3-methylisoquinolin-1-yl)bicyclo[1.1.1]pentane-1-carbonitrile (6a). The title compound was prepared according to the general procedure using 1,3dioxoisoindolin-2-yl 3-cyanobicyclo[1.1.1]pentane-1-carboxylate $(0.254 \mathrm{mmol}, 1$ equiv) as the RAE and MeCN as the solvent. The reaction was purified by automated flash chromatography $(0-100 \%$ EtOAc in DCM) and the product was identified by LCMS. Product 6a was isolated as a yellow glass (18 mg, 31\%). $1 \mathrm{H} \mathrm{NMR}\left(\mathrm{CDCl}_{3}, 400 \mathrm{MHz}\right) \delta 8.19$ $(\mathrm{d}, J=8.6 \mathrm{~Hz}, 1 \mathrm{H}), 7.75(\mathrm{~d}, J=8.2 \mathrm{~Hz}, 1 \mathrm{H}), 7.63(\mathrm{t}, J=7.6 \mathrm{~Hz}, 1 \mathrm{H}), 7.48-7.56(\mathrm{~m}, 1 \mathrm{H}), 7.41(\mathrm{~s}, 1 \mathrm{H})$, $2.88(\mathrm{~s}, 6 \mathrm{H}), 2.66(\mathrm{~s}, 3 \mathrm{H}) \mathrm{ppm} ;{ }^{13} \mathrm{C} \mathrm{NMR}\left(101 \mathrm{MHz}, \mathrm{CDCl}_{3}\right) \delta 154.6,150.8,137.1,129.7,127.0$, 
S19

126.2, 125.1, 124.9, 118.6, 118.0, 57.1, 47.4, 24.5, 24.2 ppm; HRMS Calcd for $\mathrm{C}_{16} \mathrm{H}_{14} \mathrm{~N}_{2}[\mathrm{M}+\mathrm{H}]^{+}$ 235.1230 , found 235.1228 .

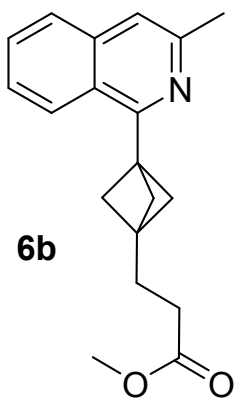

Methyl 3-(3-(3-methylisoquinolin-1-yl)bicyclo[1.1.1]pentan-1-yl)propanoate (6b). The title compound was prepared according to the general procedure using 1,3dioxoisoindolin-2-yl 3-(3-methoxy-3-oxopropyl)bicyclo[1.1.1]pentane-1-carboxylate as the RAE ( $0.254 \mathrm{mmol}, 1$ equiv) and $\mathrm{MeCN}$ as the solvent. The reaction was purified by automated flash chromatography (0-100\% EtOAc in DCM) and the product was identified by LCMS. Product $6 \mathrm{~b}$ was isolated as a yellow solid (17 mg, $23 \%$ ). $1 \mathrm{H}$ NMR $\left(\mathrm{CDCl}_{3}, 400 \mathrm{MHz}\right) \delta 8.37(\mathrm{~d}, J=8.1 \mathrm{~Hz}, 1 \mathrm{H}), 7.71(\mathrm{~d}, J=8.3 \mathrm{~Hz}, 1 \mathrm{H}), 7.59$ (ddd, $J=8.1,6.9,1.1 \mathrm{~Hz}$, 1H), 7.47 (ddd, $J=8.4,7.0,1.2 \mathrm{~Hz}, 1 \mathrm{H}$ ), $7.35(\mathrm{~s}, 1 \mathrm{H}), 3.71(\mathrm{~s}, 3 \mathrm{H}), 2.66(\mathrm{~s}, 3 \mathrm{H}), 2.42(\mathrm{t}, J=7.7 \mathrm{~Hz}$, 2H), $2.30(\mathrm{~s}, 6 \mathrm{H}), 1.99$ (t, $J=7.7 \mathrm{~Hz}, 2 \mathrm{H}) \mathrm{ppm} ;{ }^{13} \mathrm{C}$ NMR $\left(126 \mathrm{MHz}, \mathrm{CDCl}_{3}\right) \delta 174.1,157.8,150.6$, 137.2, 129.4, 126.7, 126.0, 125.5, 125.4, 117.7, 53.5, 51.6, 43.5, 40.0, 31.4, 26.9, 24.5 ppm; HRMS Calcd for $\mathrm{C}_{19} \mathrm{H}_{21} \mathrm{NO}_{2}[\mathrm{M}+\mathrm{H}]^{+}$296.1645, found 296.1647.

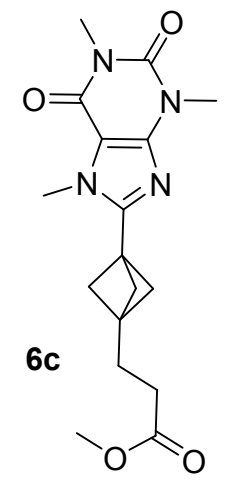

\section{Methyl}

3-(3-(1,3,7-trimethyl-2,6-dioxo-2,3,6,7-tetrahydro-1H-purin-8yl)bicyclo[1.1.1]pentan-1-yl)propanoate (6c). The title compound was prepared according to the general procedure using 1,3-dioxoisoindolin-2-yl 3-(3-methoxy-3oxopropyl)bicyclo[1.1.1]pentane-1-carboxylate as the RAE (0.254 mmol, 1 equiv) and $\mathrm{MeCN}$ as the solvent. The reaction was purified by automated flash chromatography (0-100\% EtOAc in DCM) and the product was identified by LCMS. Product $6 \mathrm{~b}$ was isolated as a yellow solid (23 mg, $27 \%$ ). $1 \mathrm{H}$ NMR (DMSO- $d_{6}, 500 \mathrm{MHz}$, TFA salt) $\delta 3.90$ (s, 3H), 3.61 (s, 3H), $3.38(\mathrm{~s}, 3 \mathrm{H}), 3.20(\mathrm{~s}, 3 \mathrm{H}), 2.35$ (t, J= $7.3 \mathrm{~Hz}, 2 \mathrm{H}), 2.11(\mathrm{~s}, 6 \mathrm{H}), 1.80$ (t, J= 7.5 $\mathrm{Hz}, 2 \mathrm{H}) \mathrm{ppm} ;{ }^{13} \mathrm{C}$ NMR (126 MHz, DMSO- $d_{6}$, TFA salt) $\delta 173.5,155.0,151.4,150.8,147.5,107.1$, 52.6, 51.8, 41.6, 35.3, 32.6, 30.9, 29.8, 28.0, 26.5 ppm; HRMS Calcd for $\mathrm{C}_{17} \mathrm{H}_{22} \mathrm{~N}_{4} \mathrm{O}_{4}[\mathrm{M}+\mathrm{H}]^{+}$ 347.1714 , found 347.1716 .

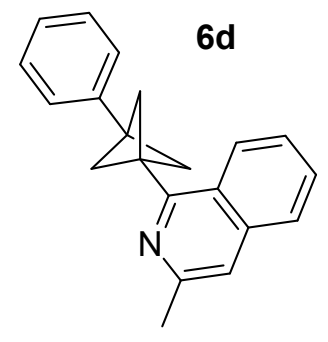

3-Methyl-1-(3-phenylbicyclo[1.1.1]pentan-1-yl)isoquinoline (6d). The title compound was prepared according to a modified general procedure whereby the RAE was generated in situ. To a $20 \mathrm{~mL}$ vial with stirbar was added 3phenylbicyclo[1.1.1]pentane-1-carboxylic acid (0.531 mmol, 1.0 equiv), $N, N-$ dimethylaminopyridine $(0.053 \mathrm{mmol}, 10 \mathrm{~mol} \%), N, N$-diisopropylcarbodiimide 
$0.531 \mathrm{mmol}, 1.0$ equiv), and and dichloromethane $(3 \mathrm{ml})$. To this solution was added $N$ hydrophthalimide $(0.551 \mathrm{mmol}, 1.1$ equiv) and the reaction mixture was left to stir at $\mathrm{rt}$ for $2.5 \mathrm{~h}$. After $2.5 \mathrm{~h}$ the reaction was concentrated under reduced pressure. With the crude RAE in hand the Minisci was performed under the general procedure using $\mathrm{MeCN}$ as the solvent, assuming $100 \%$ yield for the formation of the RAE and amounts adjusted to ensure the above described stoichiometry. The title compound purified by automated flash chromatography (0-10\% EtOAc in DCM) and the product was identified by LCMS. Product $6 \mathrm{~d}$ was isolated as a off white amorphous solid $(103 \mathrm{mg}, 68 \%)^{1} \mathrm{H}$ NMR $\left(\mathrm{CDCl}_{3}, 400 \mathrm{MHz}\right) \delta 8.49(\mathrm{~d}, J=8.7 \mathrm{~Hz}, 1 \mathrm{H}), 7.75(\mathrm{~d}, J=9.1 \mathrm{~Hz}, 1 \mathrm{H}), 7.64(\mathrm{t}, J=6.6 \mathrm{~Hz}, 1 \mathrm{H})$, $7.64(\mathrm{t}, J=6.6 \mathrm{~Hz}, 1 \mathrm{H}), 7.53(\mathrm{t}, J=7.2 \mathrm{~Hz}, 1 \mathrm{H}), 7.42-7.38(\mathrm{~m}, 5 \mathrm{H}), 7.43-7.28(\mathrm{~m}, 1 \mathrm{H}), 2.76(\mathrm{~s}, 6 \mathrm{H})$, 2.73 (s, 3H); ${ }^{13} \mathrm{C} \mathrm{NMR}\left(\mathrm{CDCl}_{3}, 100 \mathrm{MHz}\right) \delta 157.9,150.7,140.9,137.3,129.6,128.3,126.9,126.6$, 126.4, 126.0, 125.7, 125.6, 117.9, 55.6, 42.8, 42.6, 24.5. HRMS Calcd for $\mathrm{C}_{21} \mathrm{H}_{19} \mathrm{~N}[\mathrm{M}+\mathrm{H}]^{+} 286.1590$. Found: 286.1586.

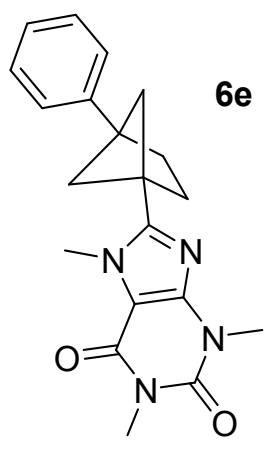

\section{1,3,7-Trimethyl-8-(4-phenylbicyclo[2.1.1]hexan-1-yl)-3,7-dihydro-1H-purine-2,6-}

dione (6e). The title compound was prepared according to a modified general procedure whereby the RAE was generated in situ. To a $20 \mathrm{~mL}$ vial with stirbar was added 4-phenylbicyclo[2.1.1] hexane-1-carboxylic acid ( $0.38 \mathrm{mmol}, 1.0$ equiv), $\mathrm{N}, \mathrm{N}-$ dimethylaminopyridine $(0.038 \mathrm{mmol}, 10 \mathrm{~mol} \%), N, N$-diisopropylcarbodiimide 0.38 mmol, 1.0 equiv), and and dichloromethane $(3 \mathrm{ml})$. To this solution was added $\mathrm{N}$ hydrophthalimide $(0.413,1.1$ equiv) and the reaction mixture was left to stir at $\mathrm{rt}$ for $2.5 \mathrm{~h}$. After $2.5 \mathrm{~h}$ the reaction was concentrated under reduced pressure. With the crude RAE in hand the Minisci was performed under the general procedure using MeCN as the solvent, assuming $100 \%$ yield for the formation of the RAE and amounts adjusted to ensure the above described stoichiometry. The title compound purified by automated flash chromatography $(0-15 \%$ EtOAc in DCM) and the product was identified by LCMS. Product $6 \mathrm{e}$ was isolated as a off white amorphous solid $(52 \mathrm{mg}$, 39\%). ${ }^{1} \mathrm{H} ~ N M R\left(\mathrm{CDCl}_{3}, 400 \mathrm{MHz}\right)$ ס 7.40-7.25 (m, 5H). 4.01 (s, 3H), $3.60(\mathrm{~s}, 3 \mathrm{H}), 3.43(\mathrm{~s}, 3 \mathrm{H}), 2.26-$ $2.14(\mathrm{~m}, 8 \mathrm{H}) ;{ }^{13} \mathrm{C} \mathrm{NMR}\left(\mathrm{CDCl}_{3}, 100 \mathrm{MHz}\right) \delta 155.4,154.9,151.8,147.9,142.4,128.4,126.6,125.9$, 107.7, 51.9, 47.0, 44.7, 34.1, 32.9, 32.5, 29.8, 27.9. HRMS Calcd for $\mathrm{C}_{20} \mathrm{H}_{22} \mathrm{~N}_{4} \mathrm{O}_{2}[\mathrm{M}+\mathrm{H}]^{+} 351.1516$. Found: 351.1821. 


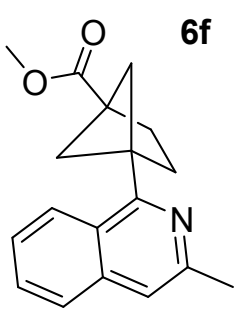

Methyl 4-(3-methylisoquinolin-1-yl)bicyclo[2.1.1]hexane-1-carboxylate (6f). The title compound was prepared according to a modified general procedure whereby the RAE was generated in situ. To a $20 \mathrm{~mL}$ vial with stirbar was added 4(methoxycarbonyl)bicyclo[2.1.1]hexane-1-carboxylic acid (0.254 mmol, 1.0 equiv), $\mathrm{N}, \mathrm{N}$-dimethylaminopyridine $(0.025 \mathrm{mmol}, 10 \mathrm{~mol} \%), \mathrm{N}, \mathrm{N}$-diisopropylcarbodiimide

$0.254 \mathrm{mmol}, 1.0$ equiv), and and dichloromethane $(3 \mathrm{ml}$,$) . To this solution was added N$ hydrophthalimide $(0.290,1.1$ equiv) and the reaction mixture was left to stir at $\mathrm{rt}$ for $2.5 \mathrm{~h}$. After $2.5 \mathrm{~h}$ the reaction was concentrated under reduced pressure. With the crude RAE in hand the Minisci was performed under the general procedure using $\mathrm{MeCN}$ as the solvent, assuming $100 \%$ yield for the formation of the RAE and amounts adjusted to ensure the above described stoichiometry. The title compound purified by automated flash chromatography (0-10\% EtOAc in DCM) and the product was identified by LCMS. Product $6 \mathrm{f}$ was isolated as an off white amorphous solid (48 $\mathrm{mg}, 67 \%)$. ${ }^{1} \mathrm{H}$ NMR $\left(\mathrm{CDCl}_{3}, 400 \mathrm{MHz}\right) \delta 8.05(\mathrm{~d}, J=8.6 \mathrm{~Hz}, 1 \mathrm{H}), 7.74(\mathrm{~d}, J=7.5 \mathrm{~Hz}, 1 \mathrm{H}), 7.59(\mathrm{t}, J=5.6 \mathrm{~Hz}, 1 \mathrm{H}), 7.46$ (t, J= 7.1 Hz, 1H), $7.35(\mathrm{~s}, 1 \mathrm{H}), 3.75(\mathrm{~s}, 3 \mathrm{H}), 2.65(\mathrm{~s}, 3 \mathrm{H}), 2.38-2.30(\mathrm{~m}, 4 \mathrm{H}), 2.25(\mathrm{~s}, 4 \mathrm{H}) ;{ }^{13} \mathrm{C}$ NMR $\left(\mathrm{CDCl}_{3}, 100 \mathrm{MHz}\right) \delta 173.9,161.0,150.1,137.8,134.3,129.5,127.0,125.7,124.6,123.6,54.8,52.2$, 48.5, 46.9, 34.0, 31.3, 24.3. HRMS Calcd for $\mathrm{C}_{18} \mathrm{H}_{19} \mathrm{NO}_{2}[\mathrm{M}+\mathrm{H}]^{+}$282.1489. Found: 282.1492.

$6 \mathrm{~g}$ 1-Cyclopropyl-3-methylisoquinoline (6g). The title compound was prepared according

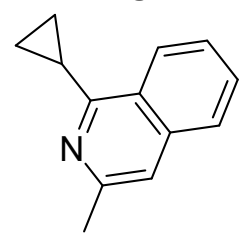
to the general procedure using the 1,3-dioxoisoindolin-2-yl cyclopropanecarboxylate ( $0.35 \mathrm{mmol}, 1$ equiv) as the RAE and $\mathrm{MeCN}$ as the solvent. The reaction was purified by automated flash chromatography (0-10\% EtOAc in DCM) and the product was identified by LCMS. Product $6 \mathrm{~g}$ was isolated as an off white amorphous solid (56 mg, 88\%). ${ }^{1} \mathrm{H}$ NMR $\left(\mathrm{CDCl}_{3}, 400 \mathrm{MHz}\right) \delta 8.41(\mathrm{~d}, J=8.3 \mathrm{~Hz}, 1 \mathrm{H}), 7.72(\mathrm{~d}, J=7.9 \mathrm{~Hz}, 1 \mathrm{H}), 7.63(\mathrm{t}, J=6.1 \mathrm{~Hz}, 1 \mathrm{H}), 7.55$ $(\mathrm{t}, J=7.1 \mathrm{~Hz}, 1 \mathrm{H}), 7.29(\mathrm{~s}, 1 \mathrm{H}), 2.79-2.69(\mathrm{~m}, 1 \mathrm{H}), 2.63(\mathrm{~s}, 3 \mathrm{H}), 1.32-1.23(\mathrm{~m}, 2 \mathrm{H}), 1.12-1.08(\mathrm{~m}$, $2 \mathrm{H}) ;{ }^{13} \mathrm{C} \mathrm{NMR}\left(\mathrm{CDCl}_{3}, 100 \mathrm{MHz}\right) \delta 160.0,149.9,136.8,129.5,126.6,125.9,125.8,125.0,116.3$, 24.9, 14.0, 8.1; HRMS Calcd for $\mathrm{C}_{13} \mathrm{H}_{13} \mathrm{~N}[\mathrm{M}+\mathrm{H}]^{+}$184.1121. Found: 184.1119.

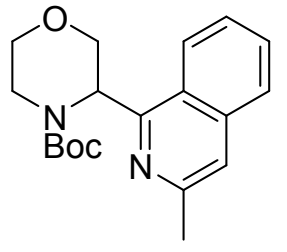

tert-Butyl 3-(3-methylisoquinolin-1-yl)morpholine-4-carboxylate (6h). The title compound was prepared according to the general procedure using the 4-(tert$6 \mathrm{~h}$ butyl) 3-(1,3-dioxoisoindolin-2-yl) morpholine-3,4-dicarboxylate $(0.254 \mathrm{mmol}, 1$ equiv) as the RAE and $M e C N$ as the solvent. The reaction was purified by 
automated flash chromatography (0-10\% EtOAc in DCM) and the product was identified by LCMS. Product $6 \mathrm{~h}$ was isolated as an off white amorphous solid $(64 \mathrm{mg}, 77 \%) .{ }^{1} \mathrm{H} \mathrm{NMR}\left(\mathrm{CDCl}_{3}, 400 \mathrm{MHz}\right)$ $\delta 7.96(\mathrm{~d}, J=8.5 \mathrm{~Hz}, 1 \mathrm{H}), 7.73(\mathrm{~d}, J=7.8 \mathrm{~Hz}, 1 \mathrm{H}), 7.60(\mathrm{t}, J=6.5 \mathrm{~Hz}, 1 \mathrm{H}), 7.49(\mathrm{t}, J=7.1 \mathrm{~Hz}, 1 \mathrm{H})$, $7.35(\mathrm{~s}, 1 \mathrm{H}), 5.76(\mathrm{bs}, 1 \mathrm{H}), 4.38-4.35(\mathrm{~m}, 1 \mathrm{H}), 4.32-4.24(\mathrm{~m}, 1 \mathrm{H}), 4.11-4.07(\mathrm{~m}, 1 \mathrm{H}), 4.03-4.00(\mathrm{~m}$, $1 \mathrm{H}), 3.84-3.79(\mathrm{~m}, 1 \mathrm{H}), 3.70-3.63(\mathrm{~m}, 1 \mathrm{H}), 2.63(\mathrm{~s}, 3 \mathrm{H}), 1.25(\mathrm{bs}, 9 \mathrm{H}) ;{ }^{13} \mathrm{C} \mathrm{NMR}\left(\mathrm{CDCl}_{3}, 100 \mathrm{MHz}\right)$ ठ 157.9, 156.5, 150.0, 137.4, 129.4, 127.3, 126.1, 123.7, 123.5, 117.7, 79.7, 69.7, 67.1, 53.5, 42.6, 28.2, 24.4; HRMS Calcd for $\mathrm{C}_{19} \mathrm{H}_{24} \mathrm{~N}_{2} \mathrm{O}_{3}[\mathrm{M}+\mathrm{H}]^{+}$329.1860. Found: 329.1856. 
${ }^{1} \mathrm{H}$ and ${ }^{13} \mathrm{C}$ NMR spectra of selected compounds 


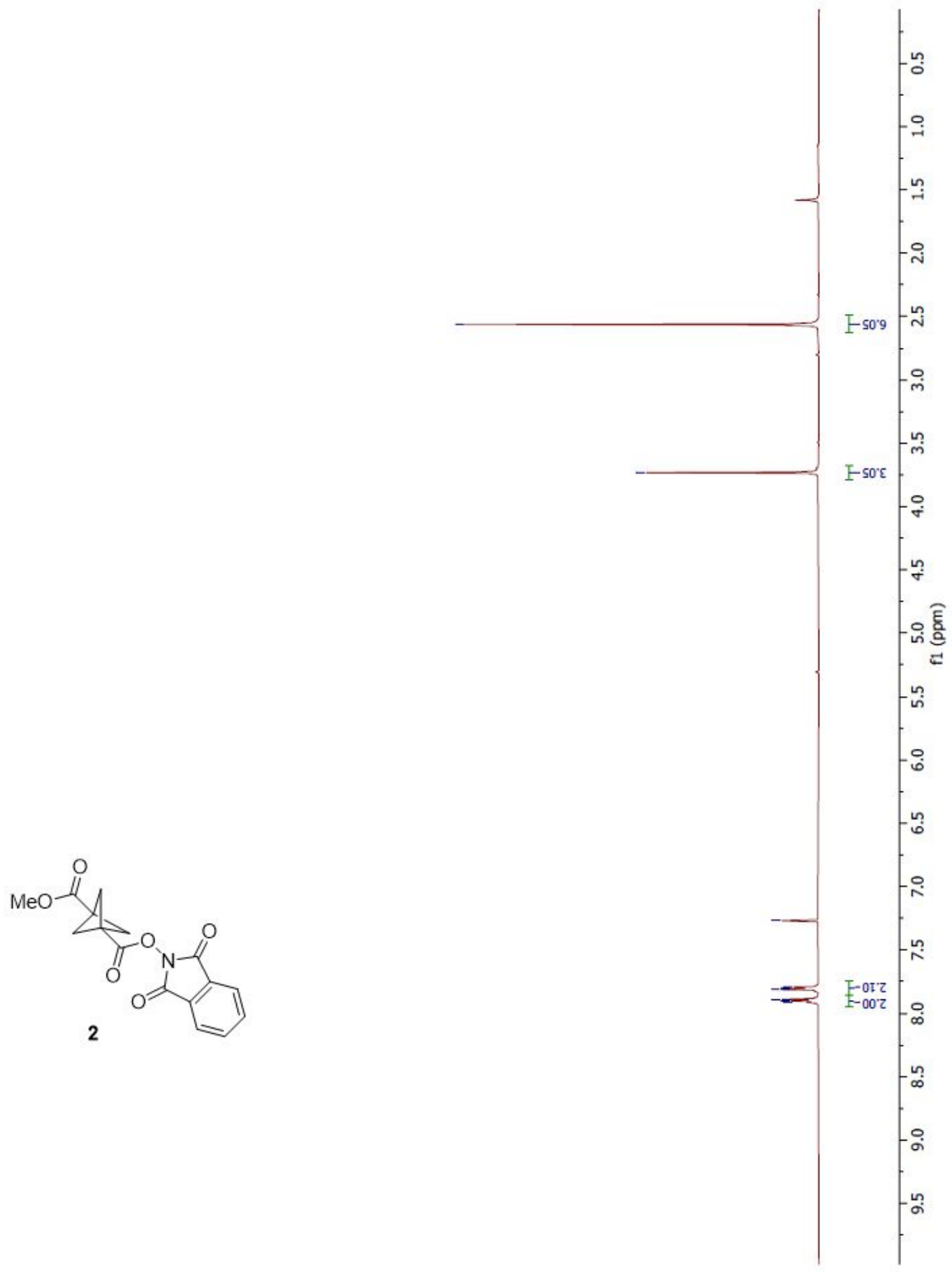



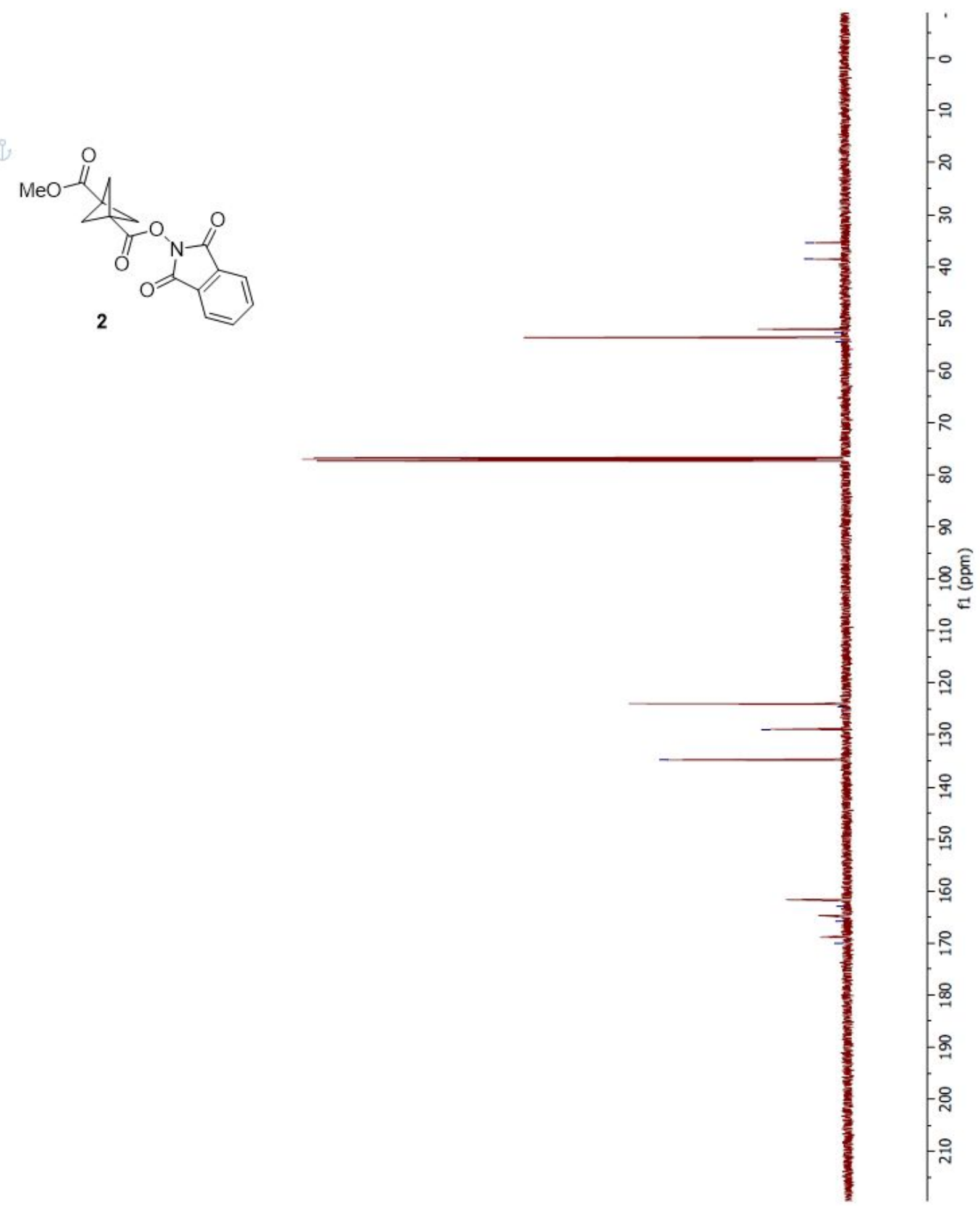


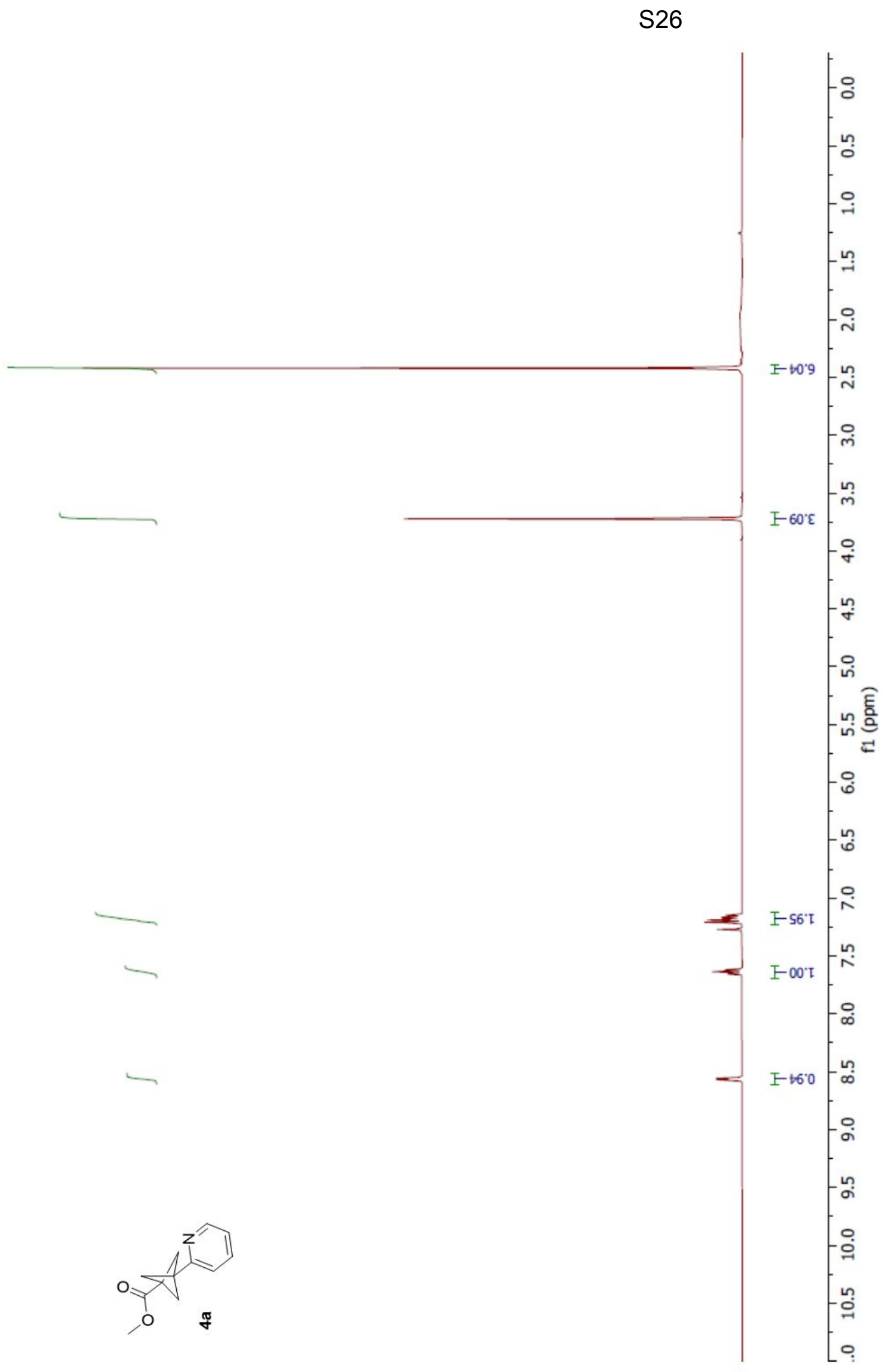



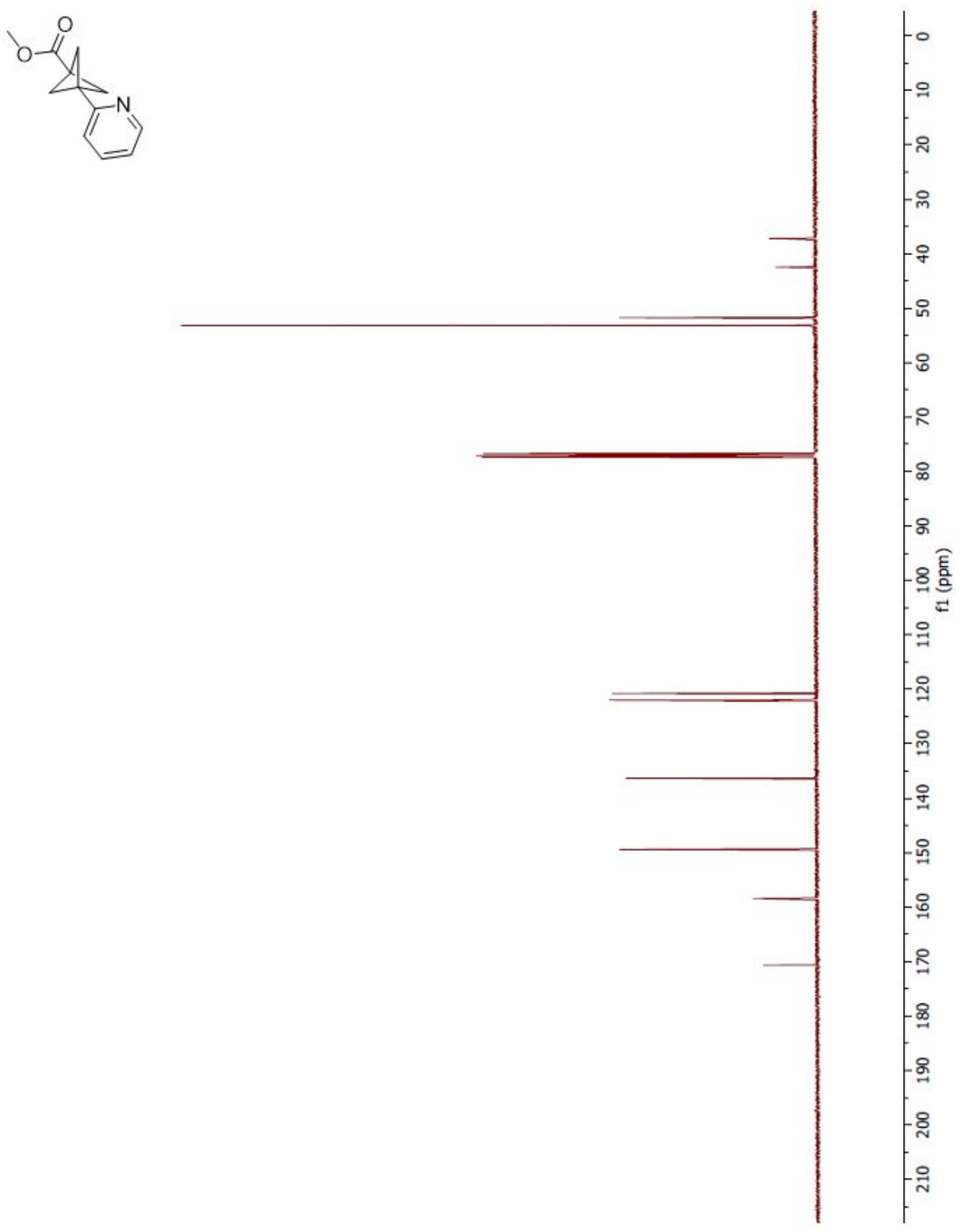


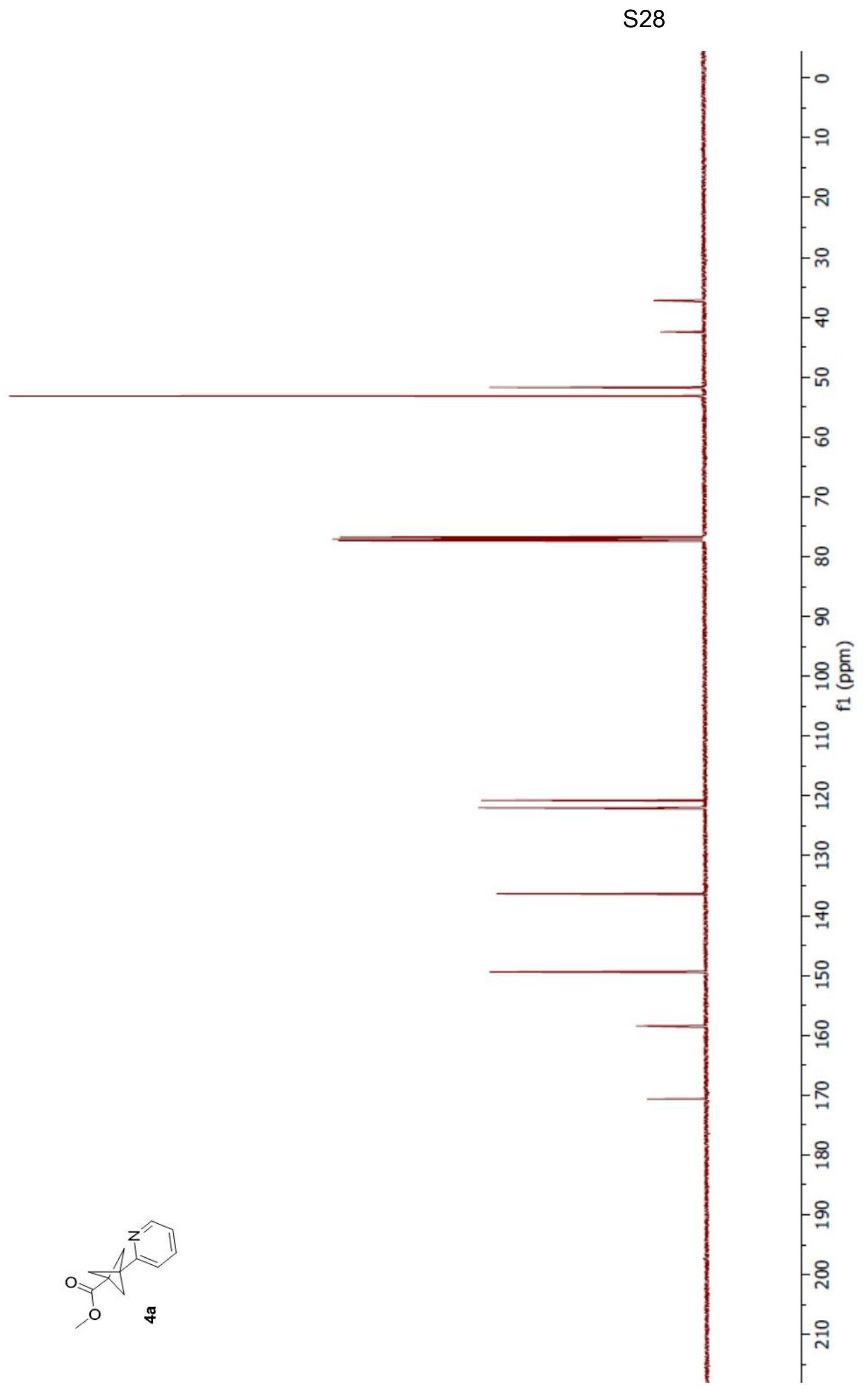



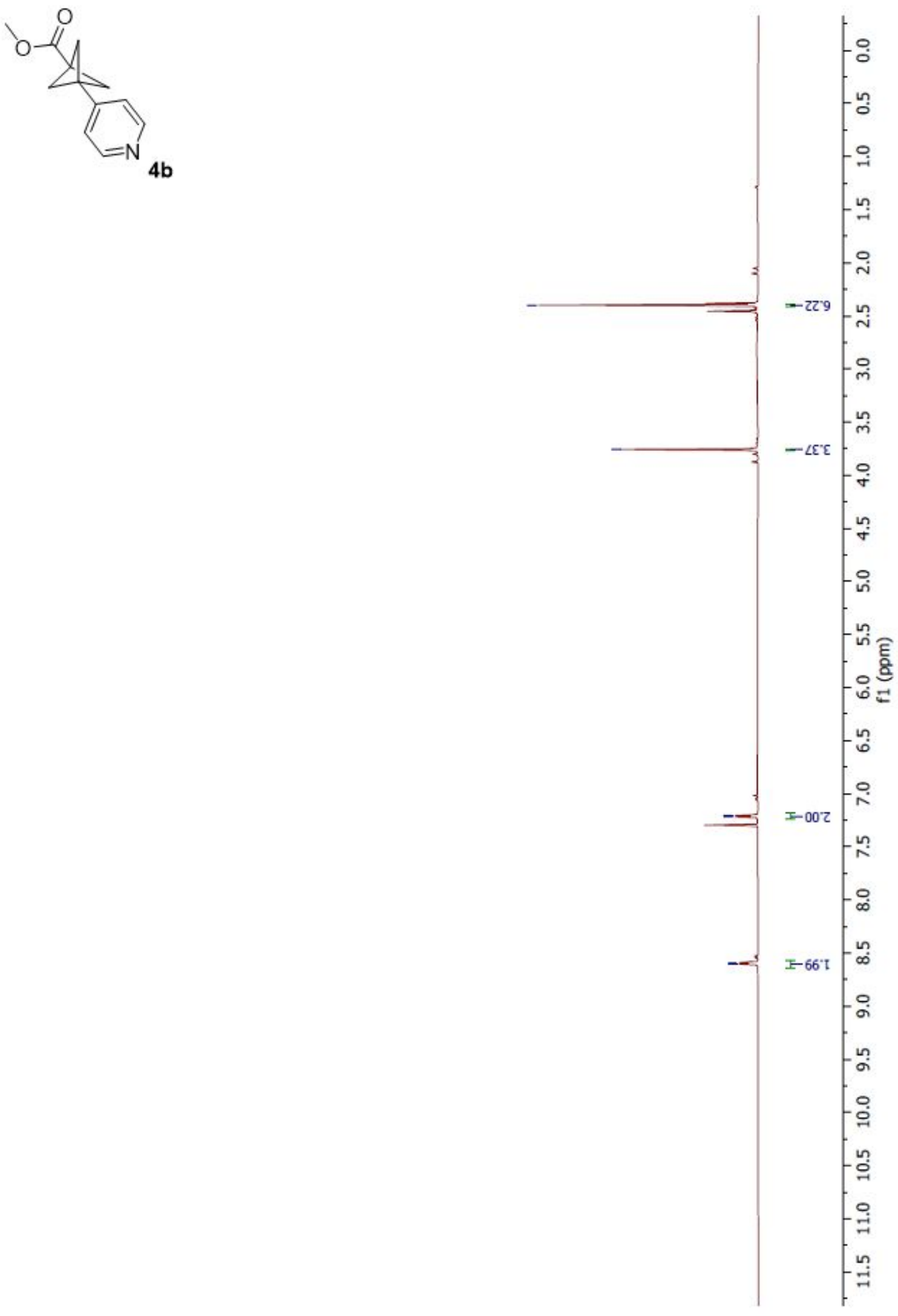

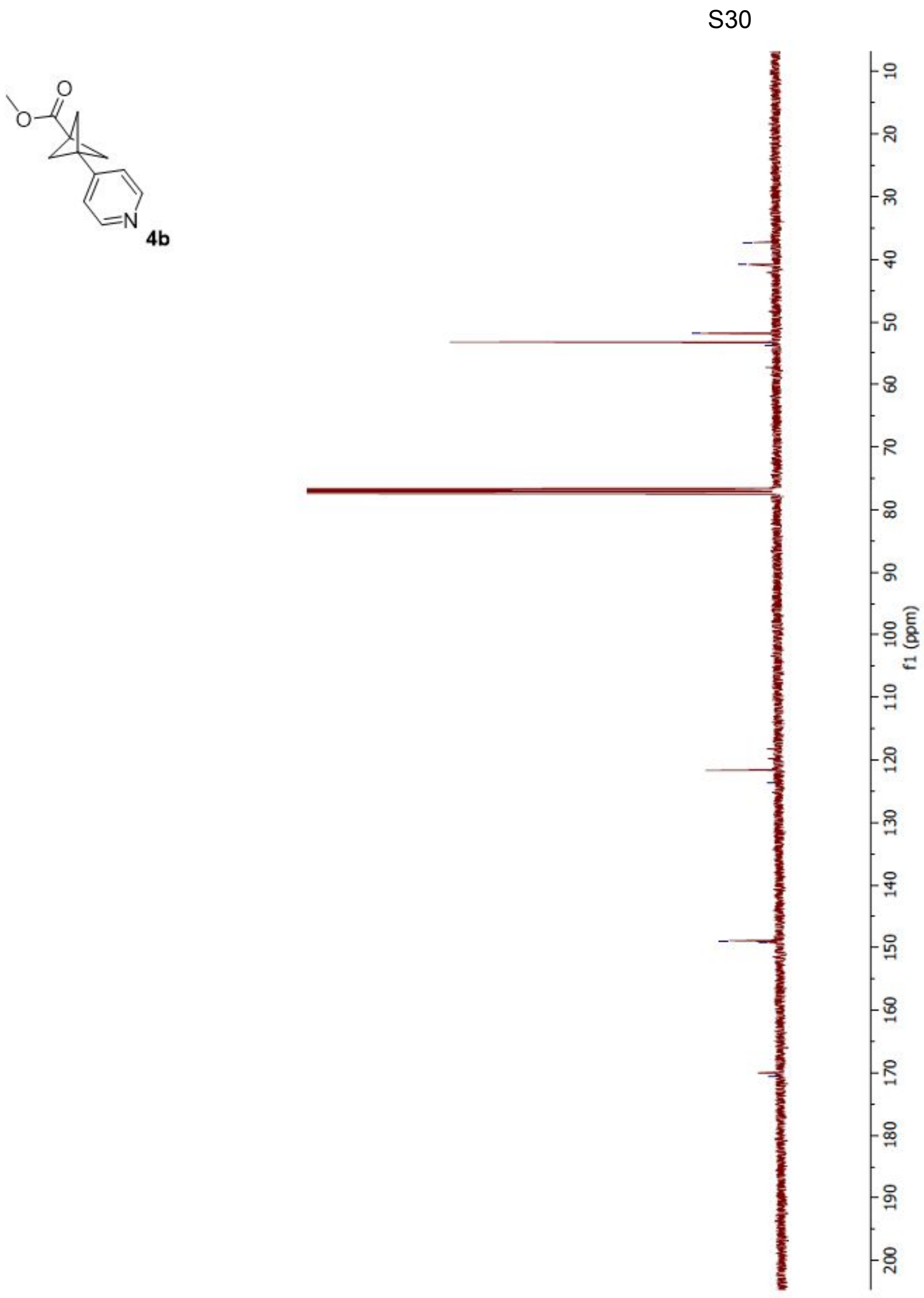


\section{S31}
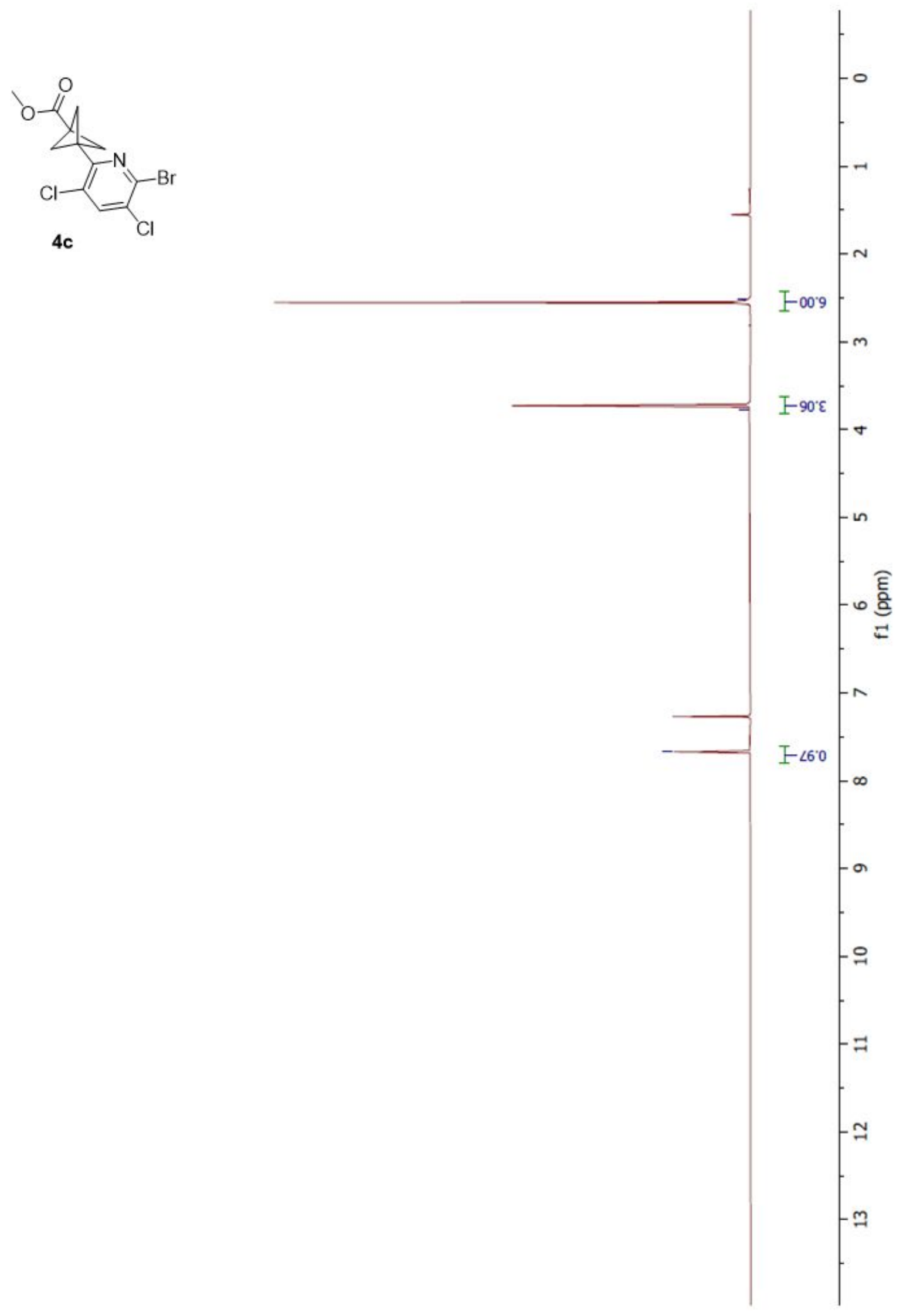

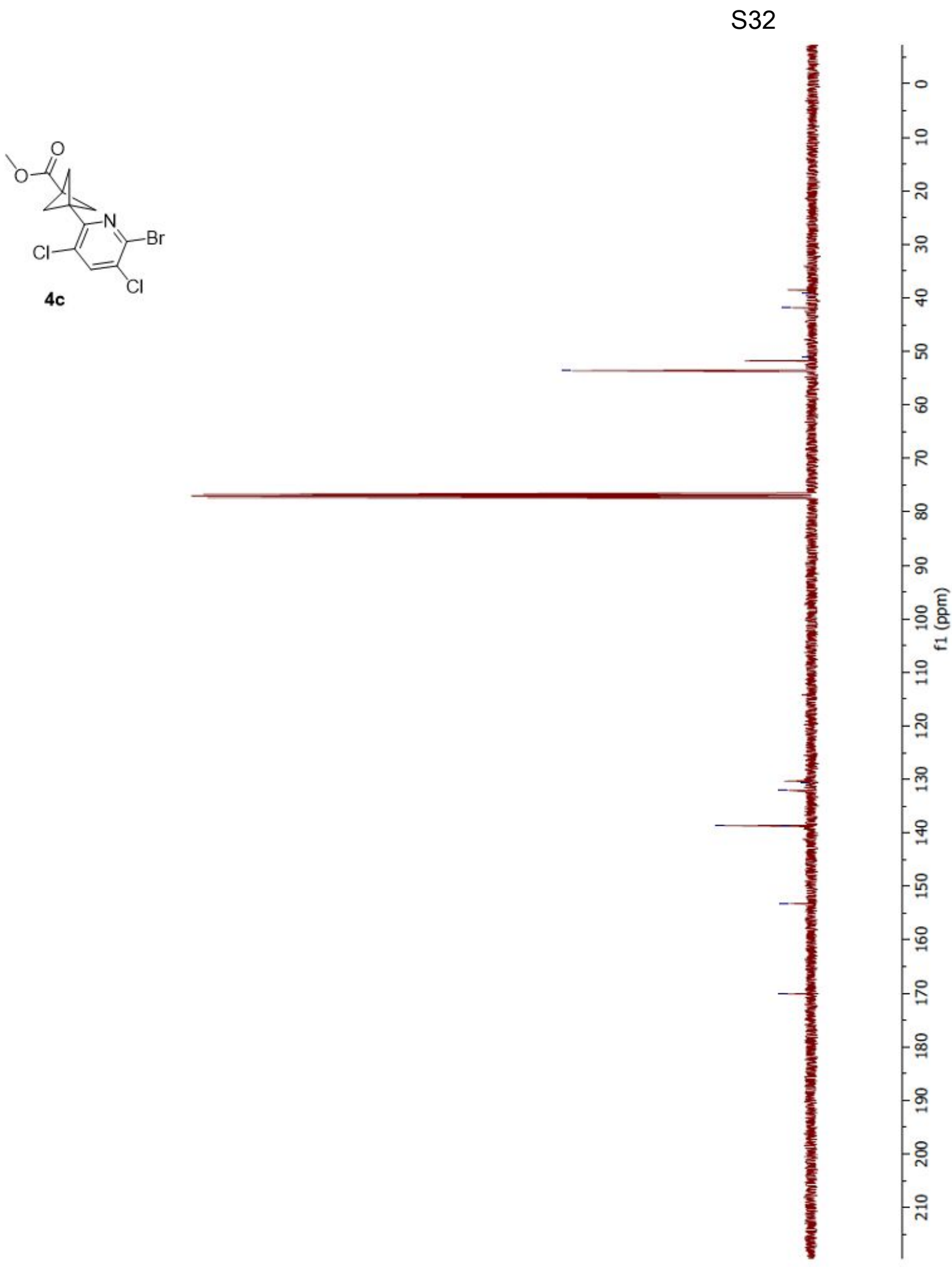

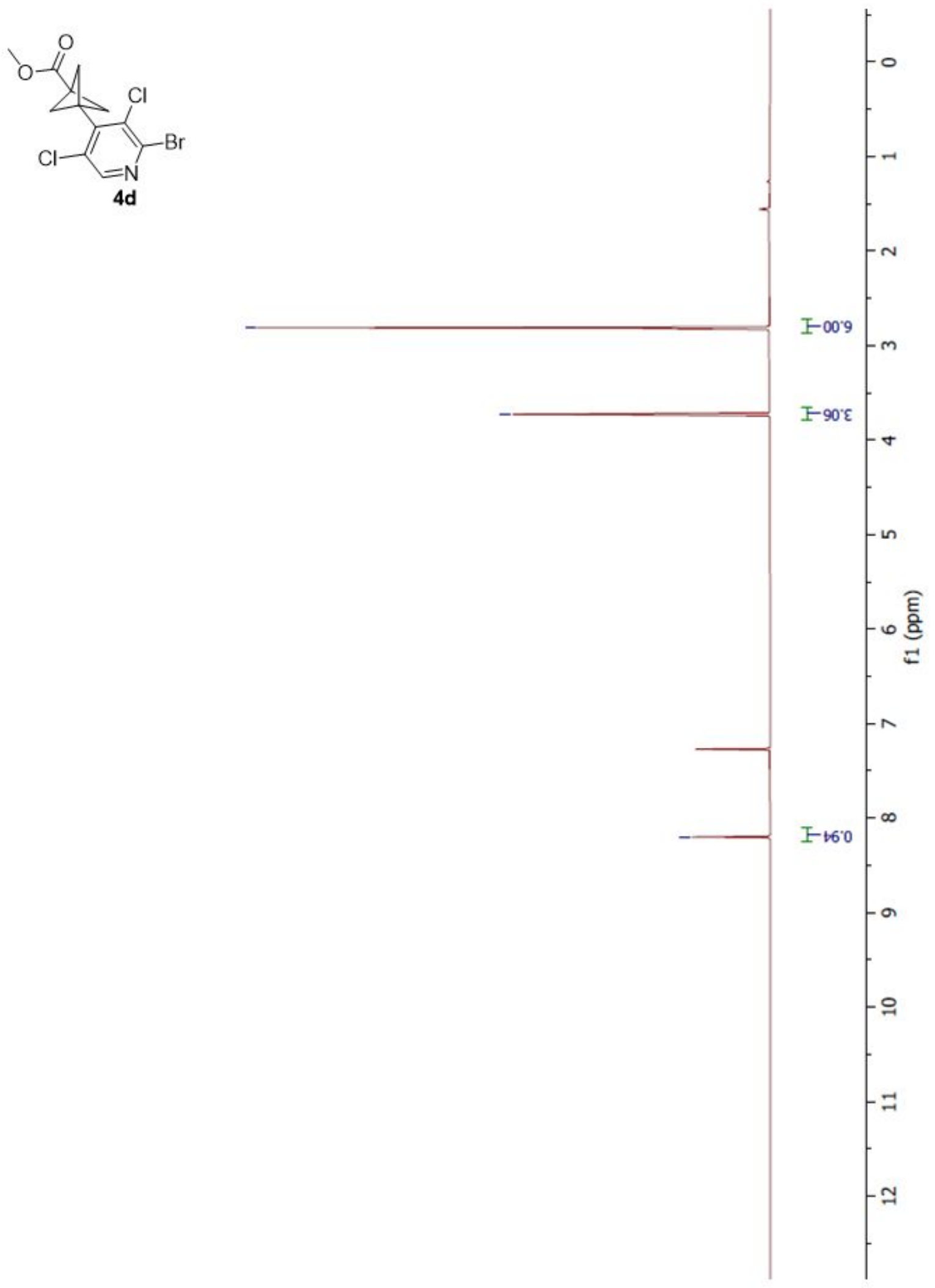


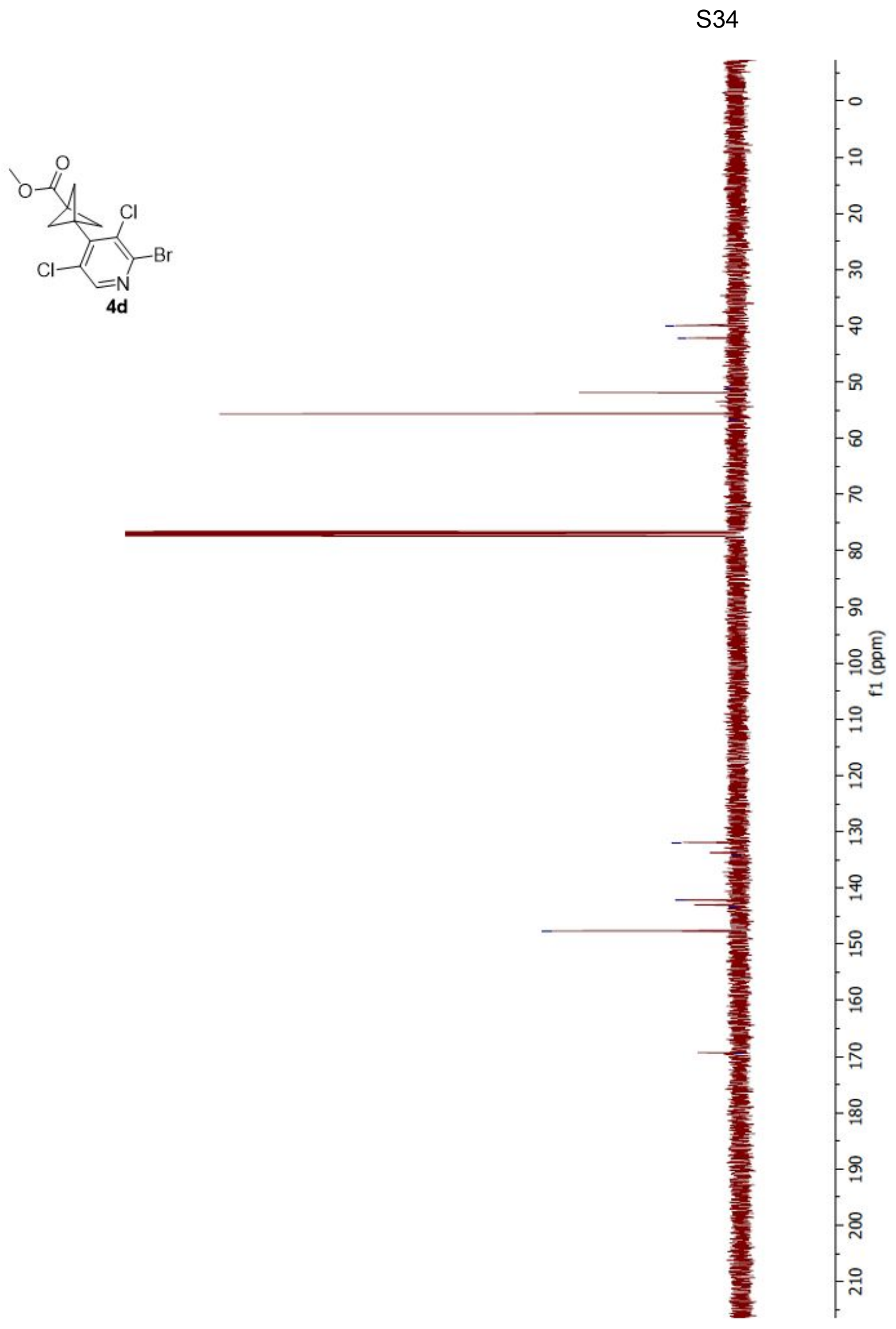




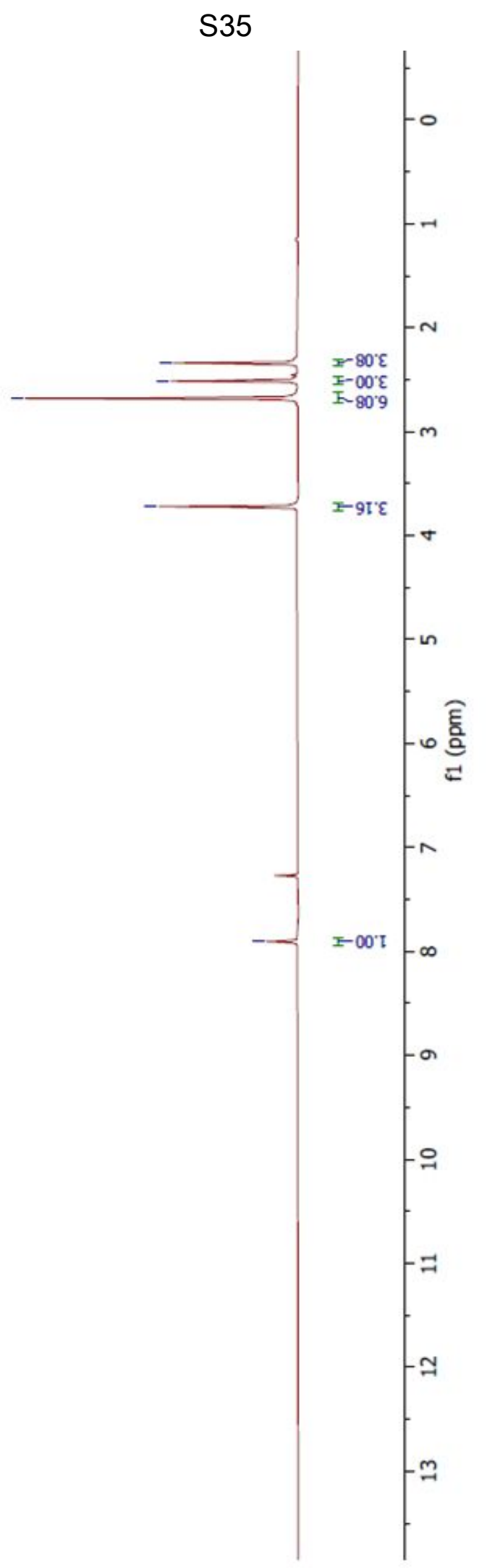




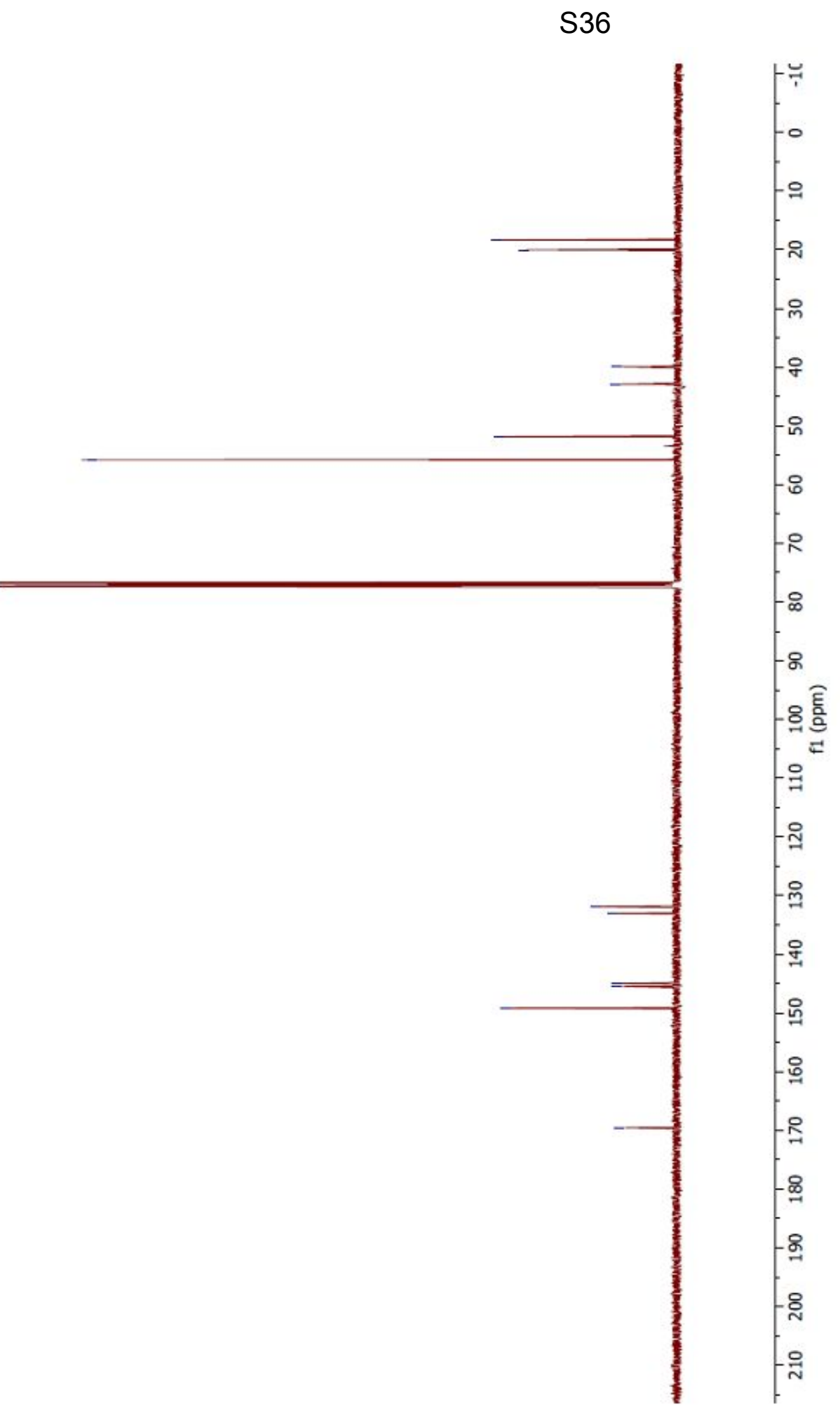




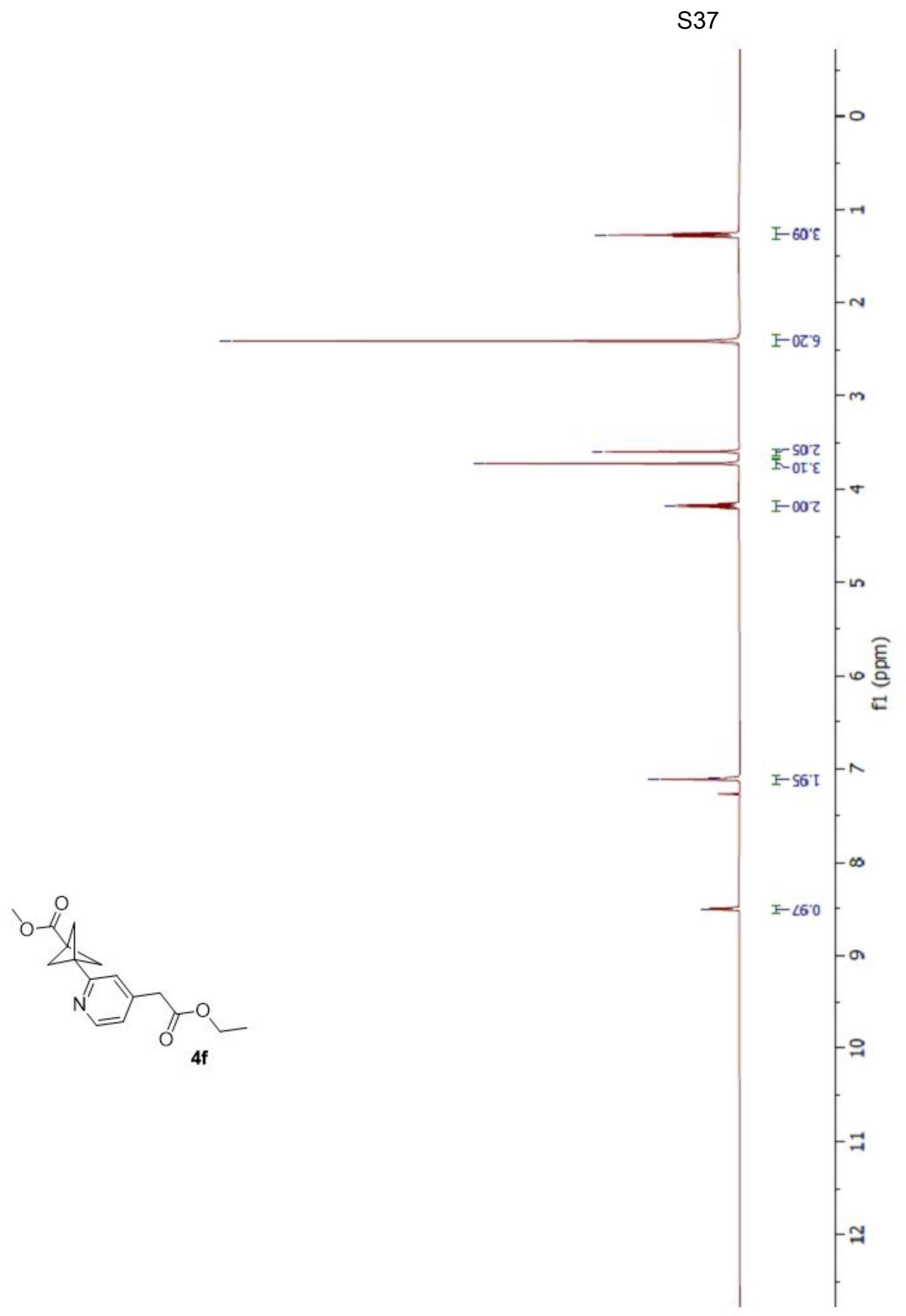




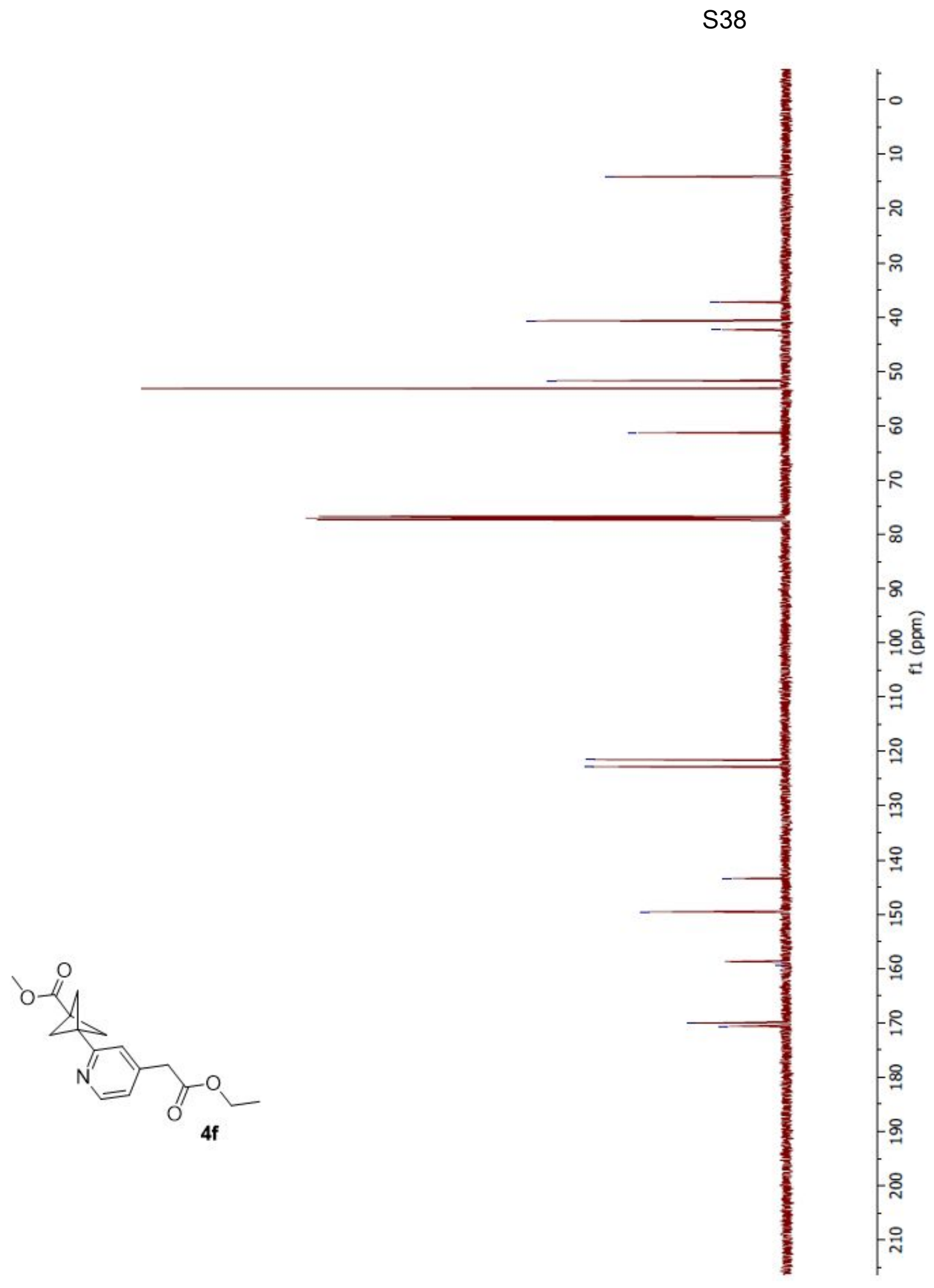


S39

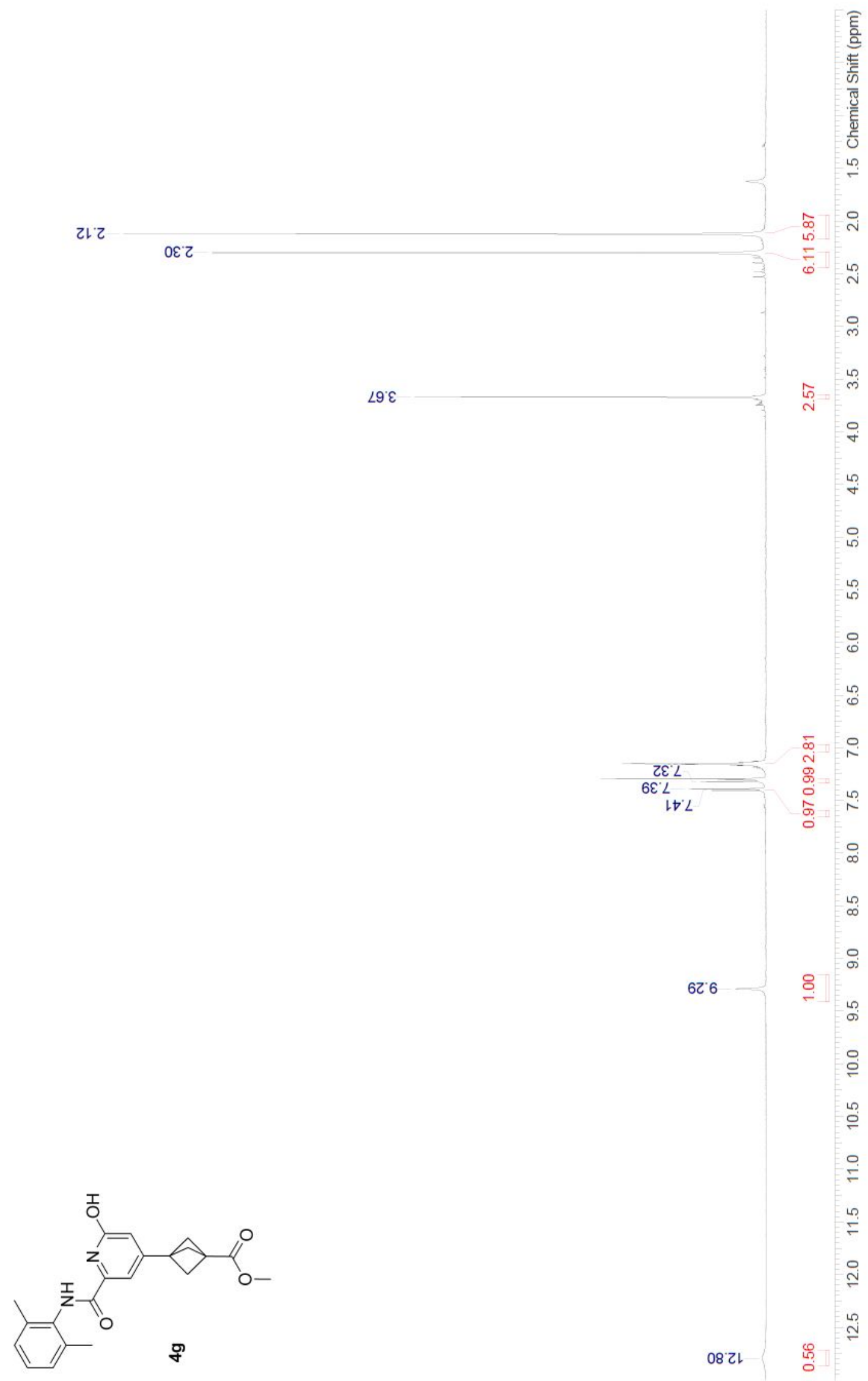




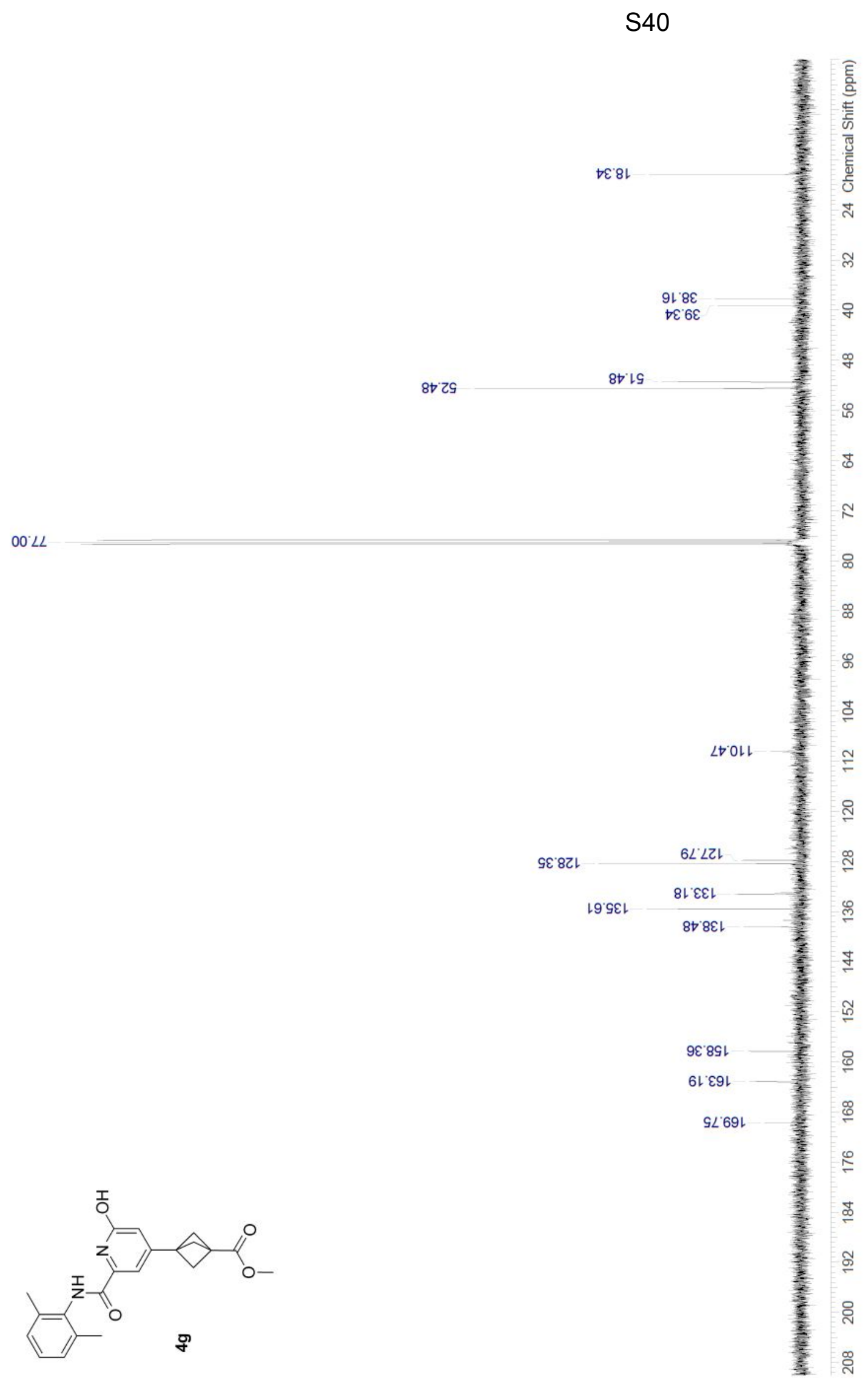




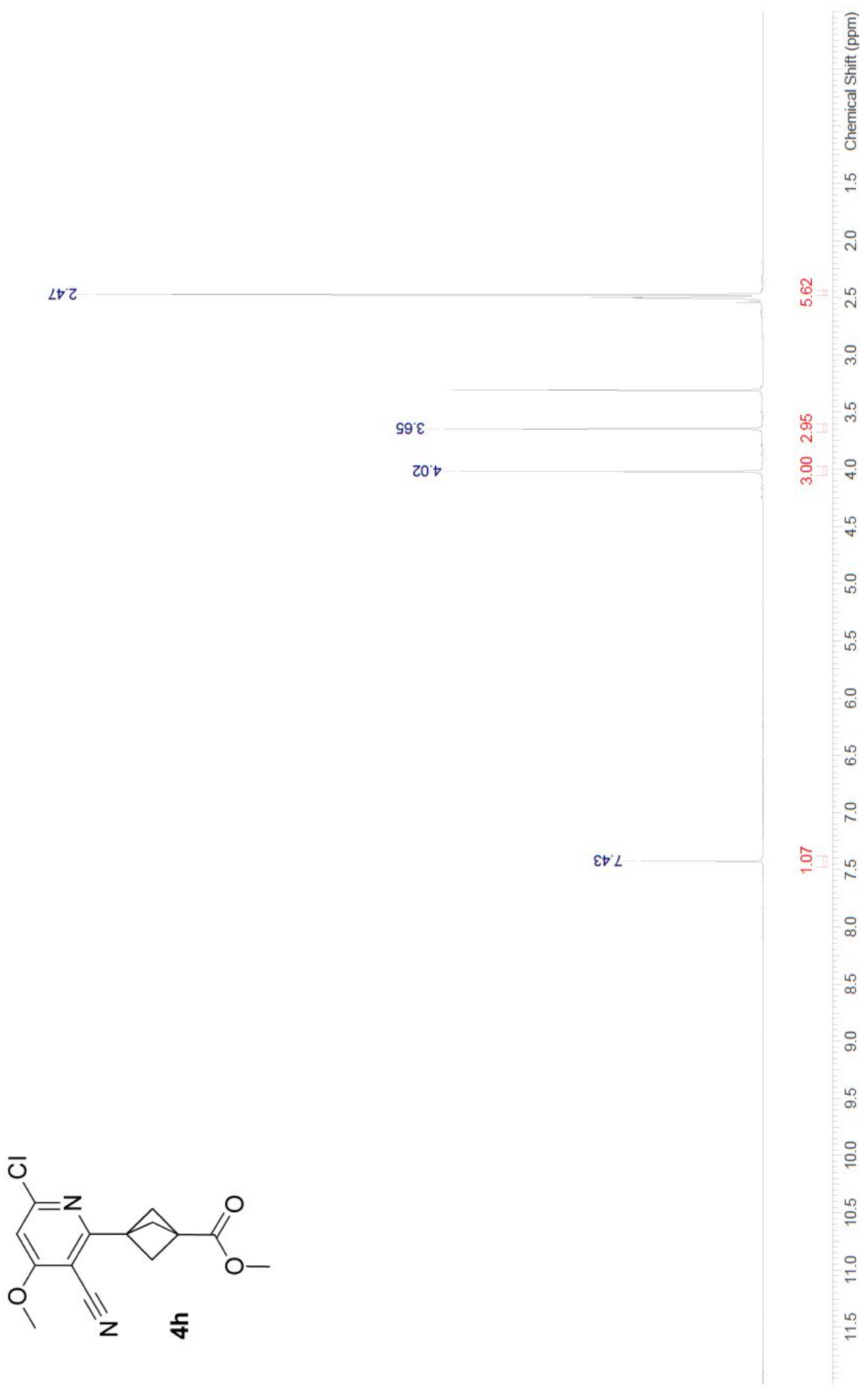




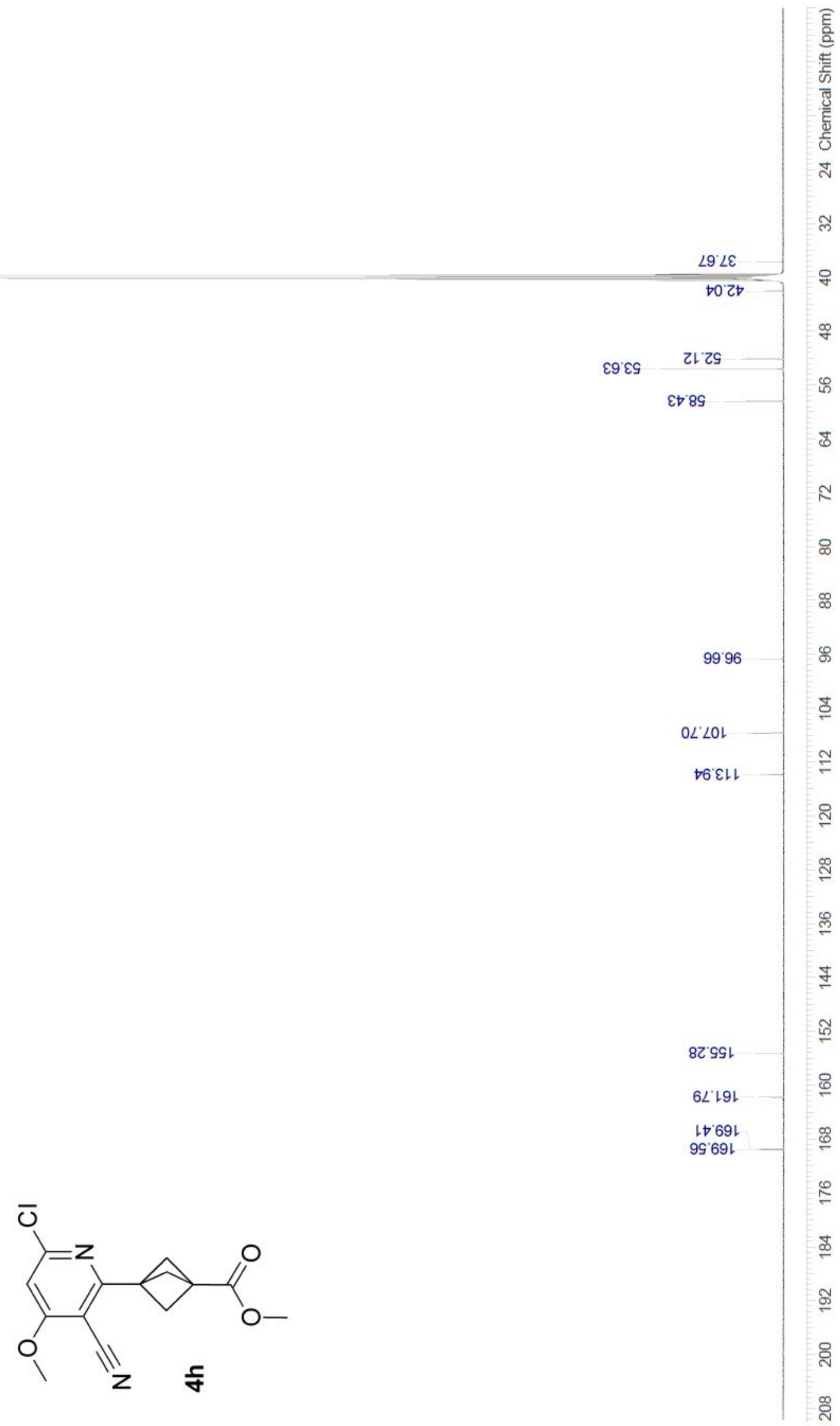


543

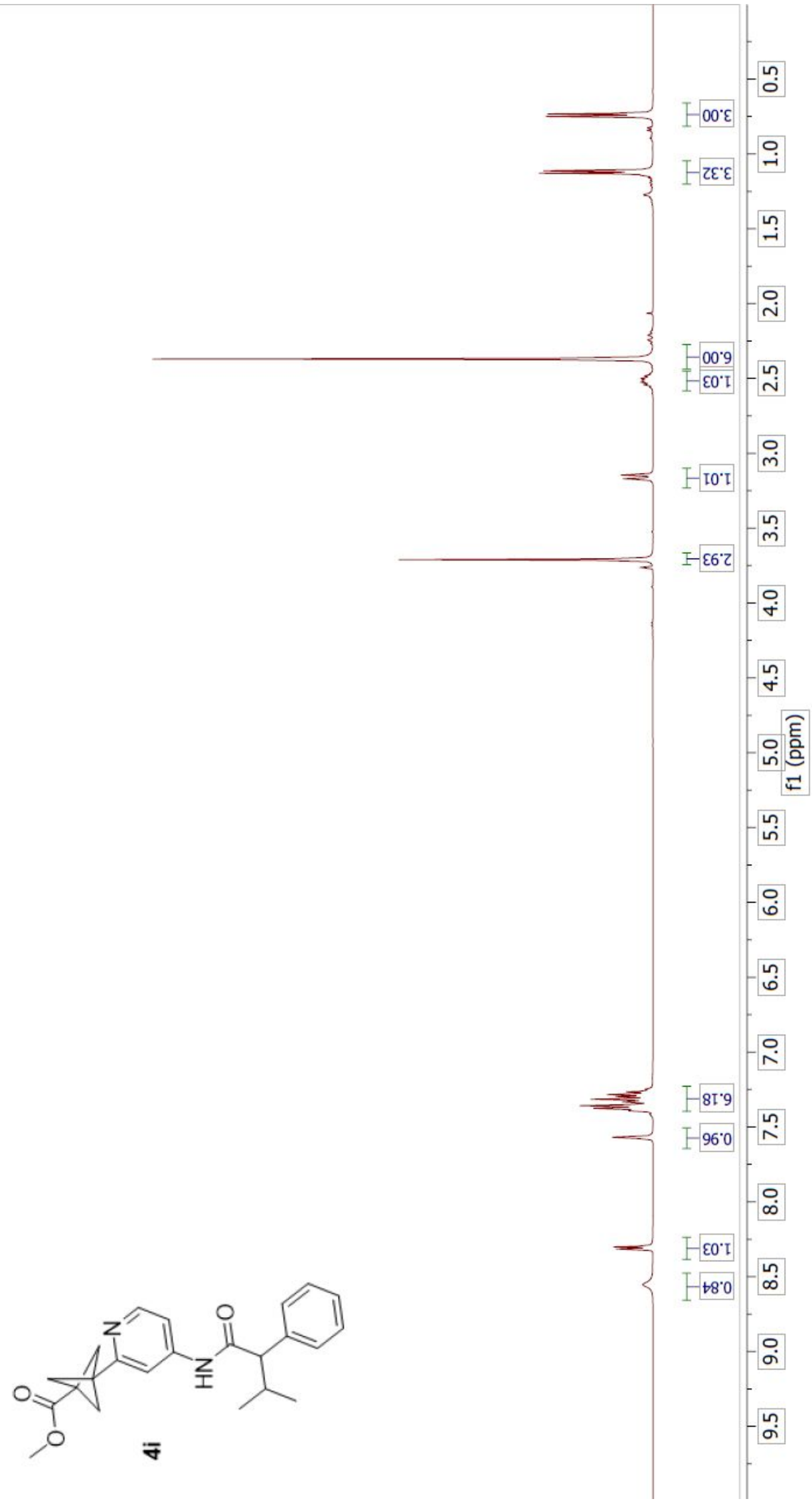




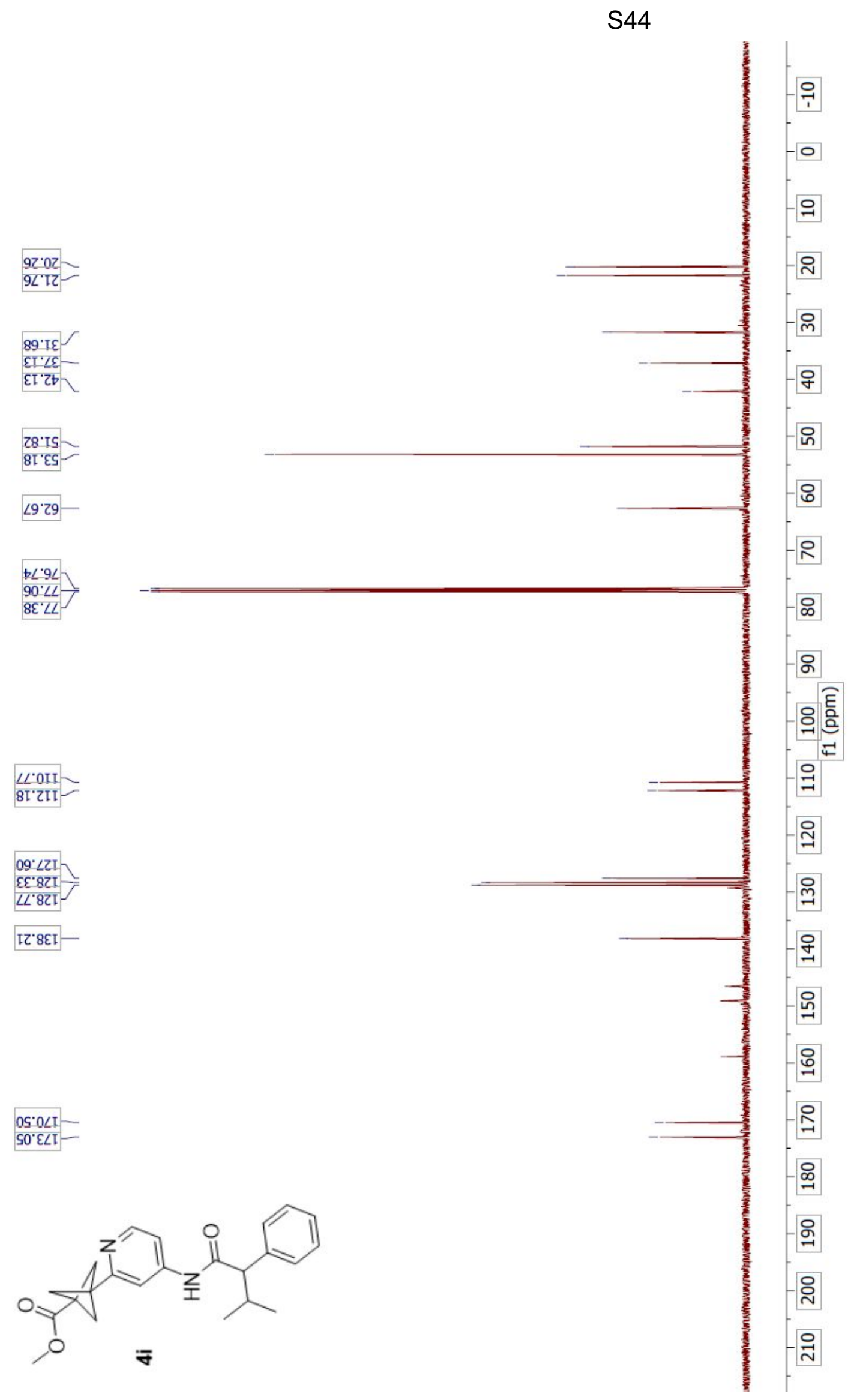




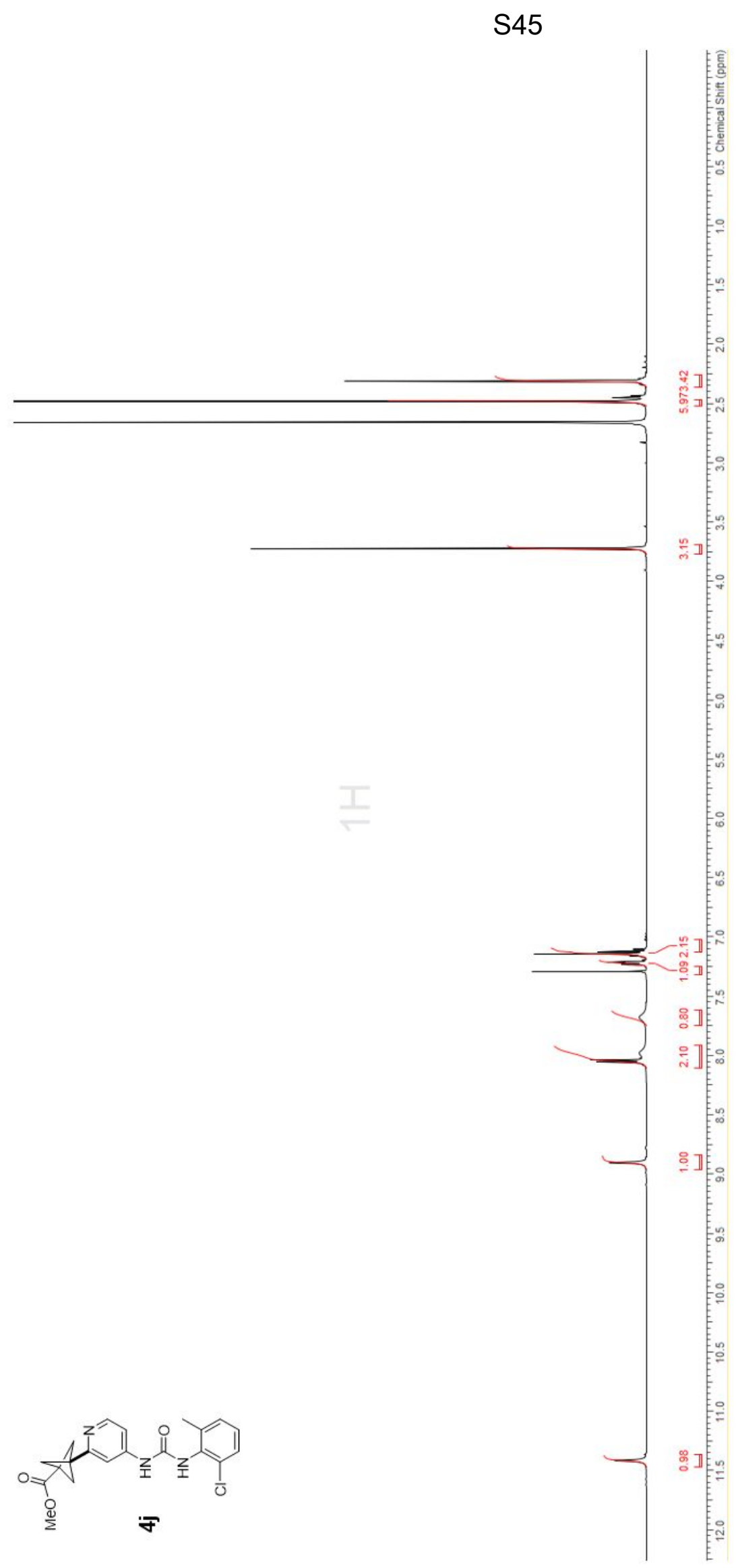




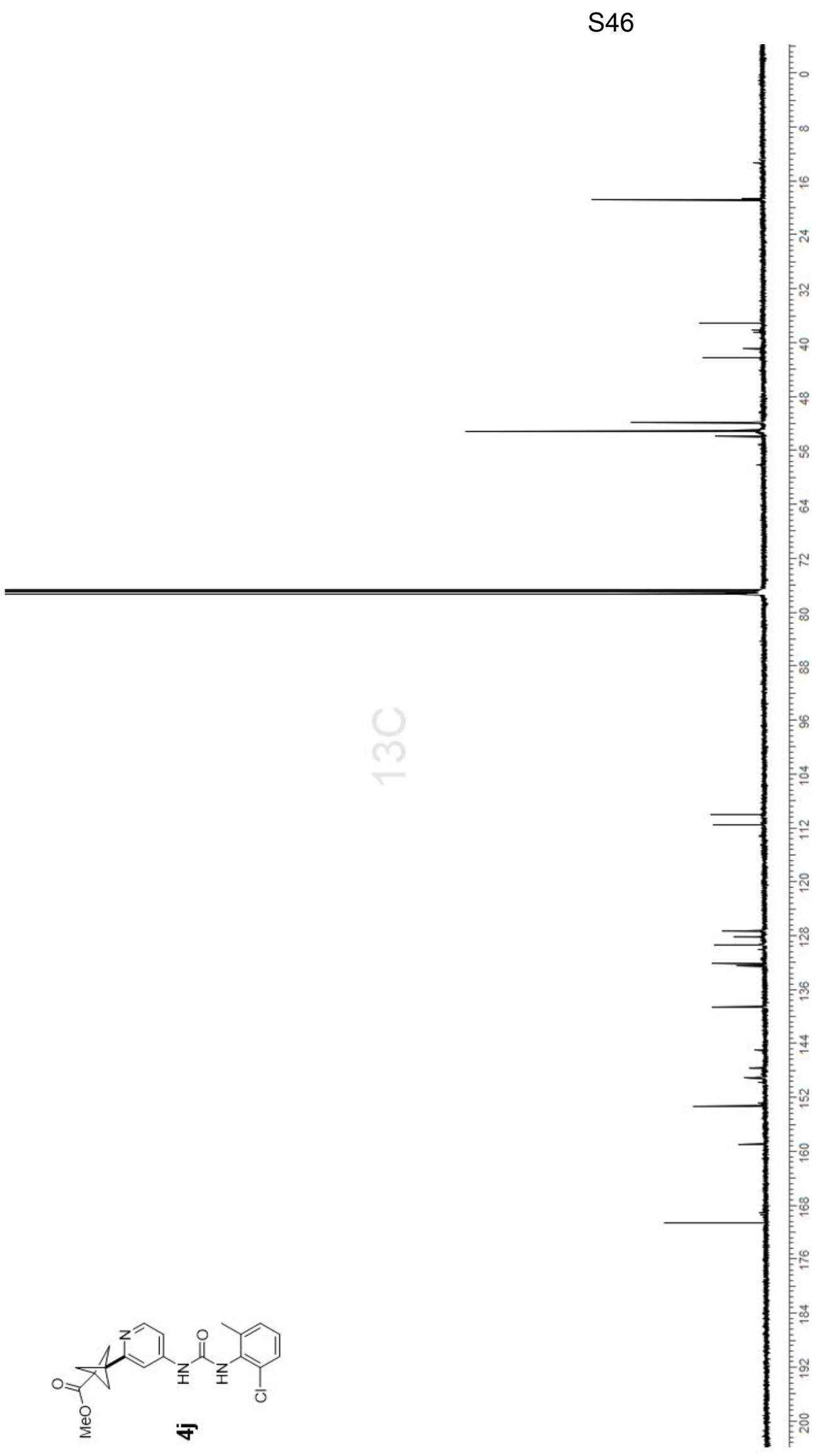




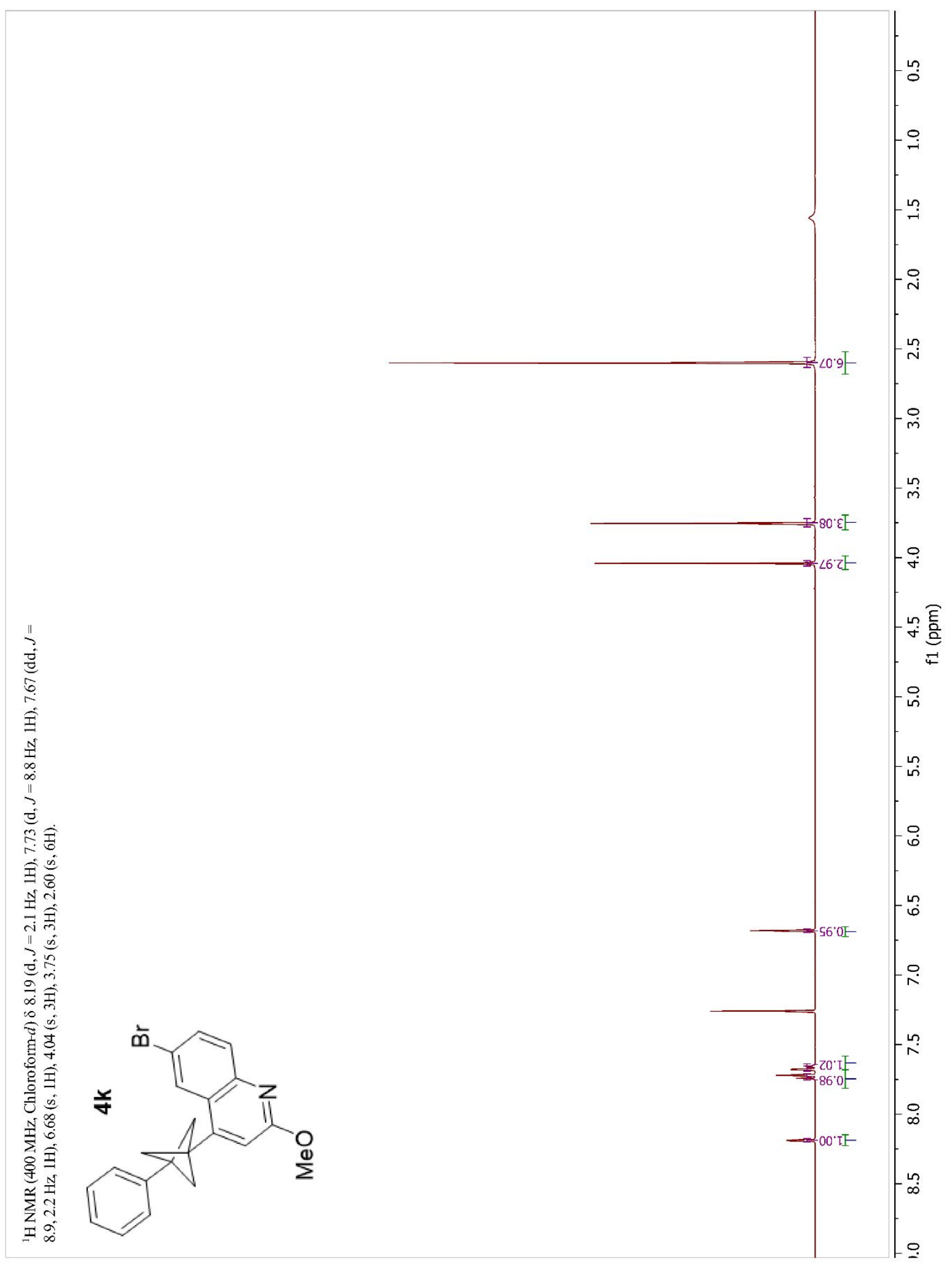




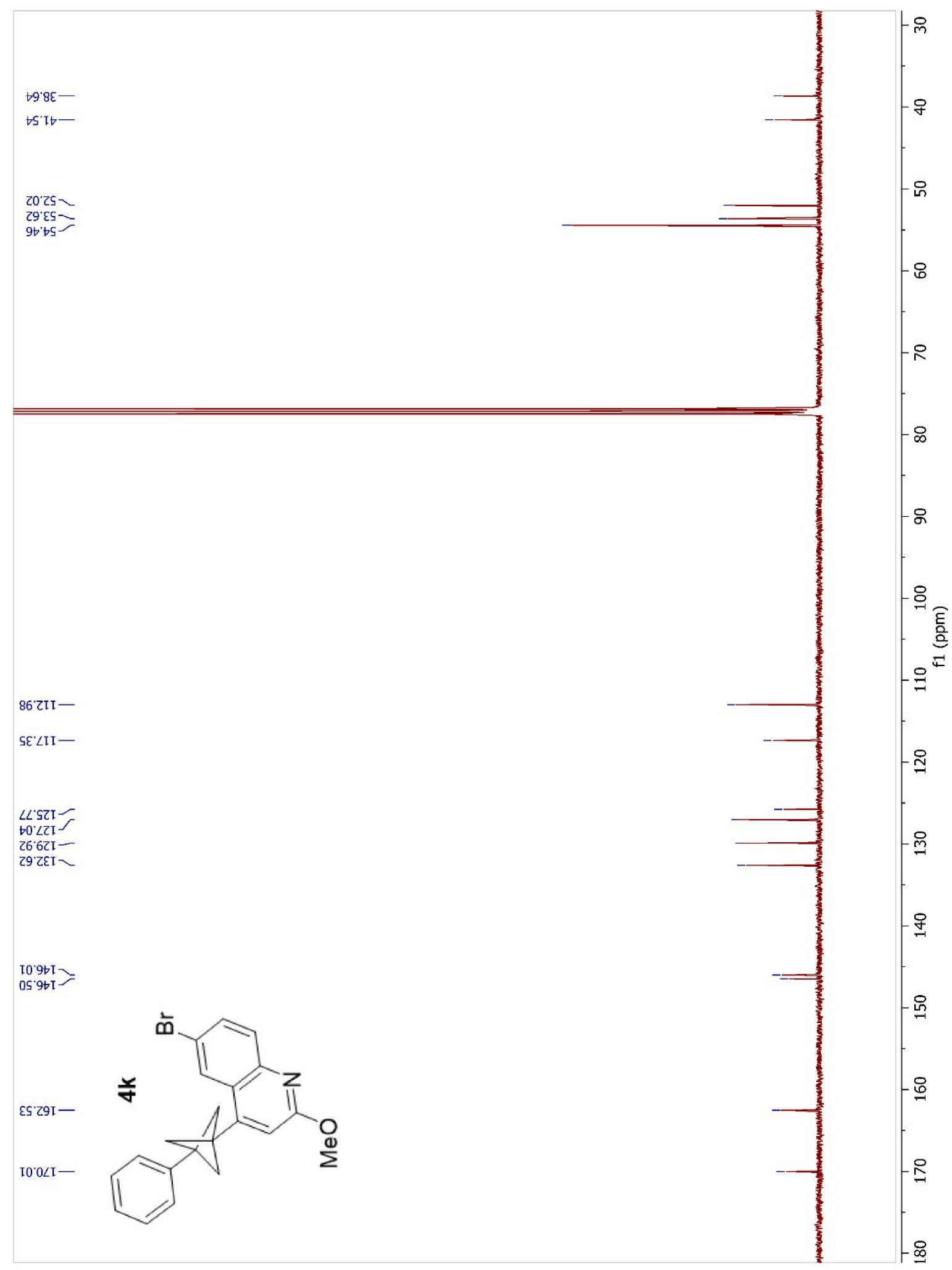




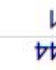

$S L \mathcal{E}$

EO"

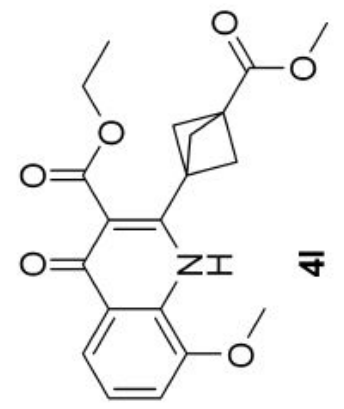




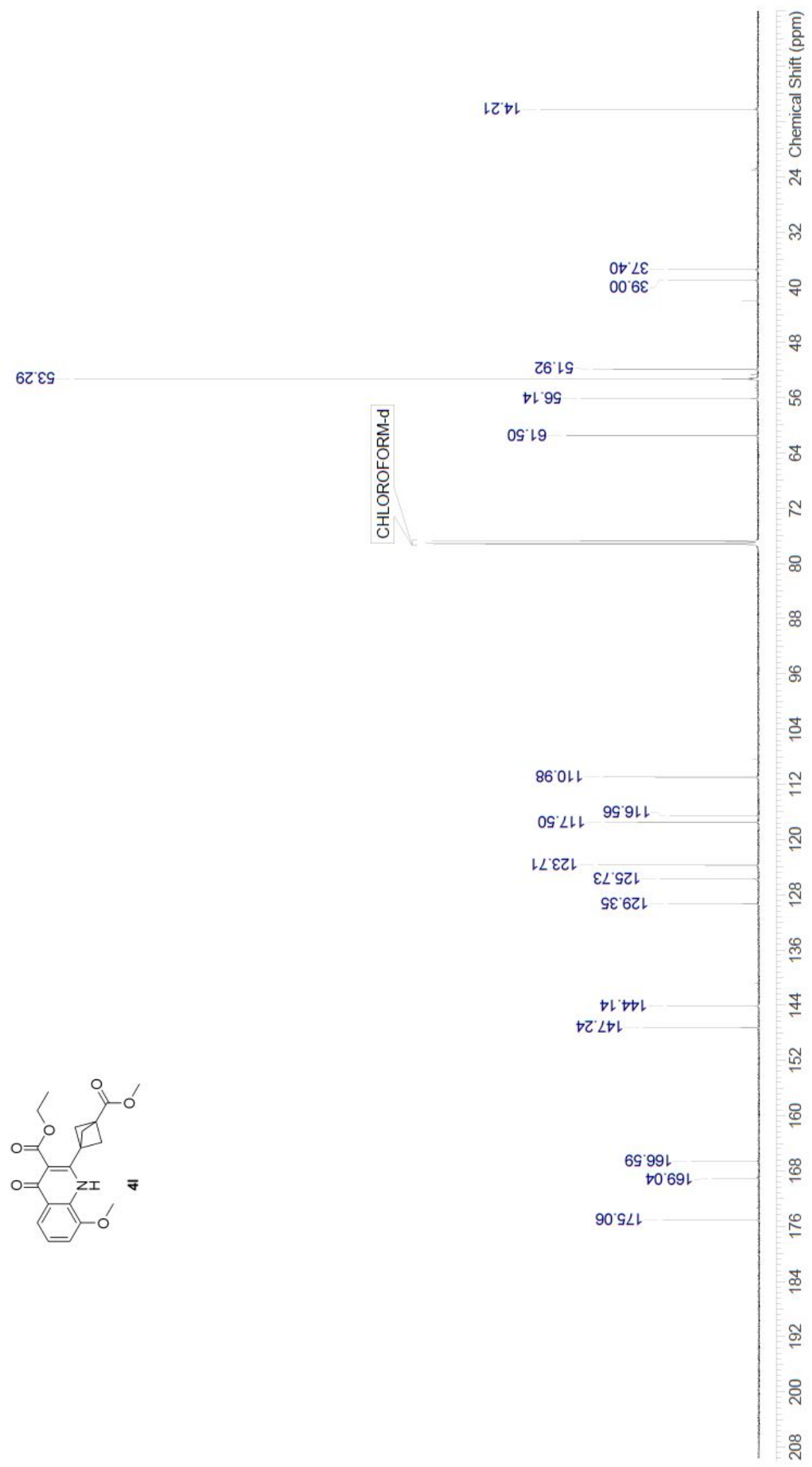




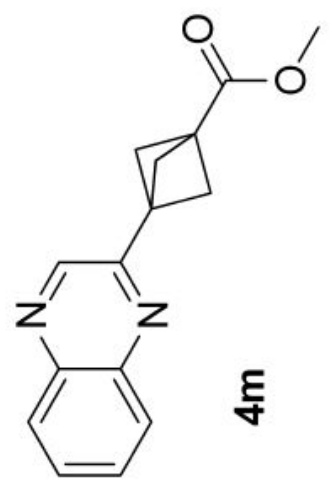




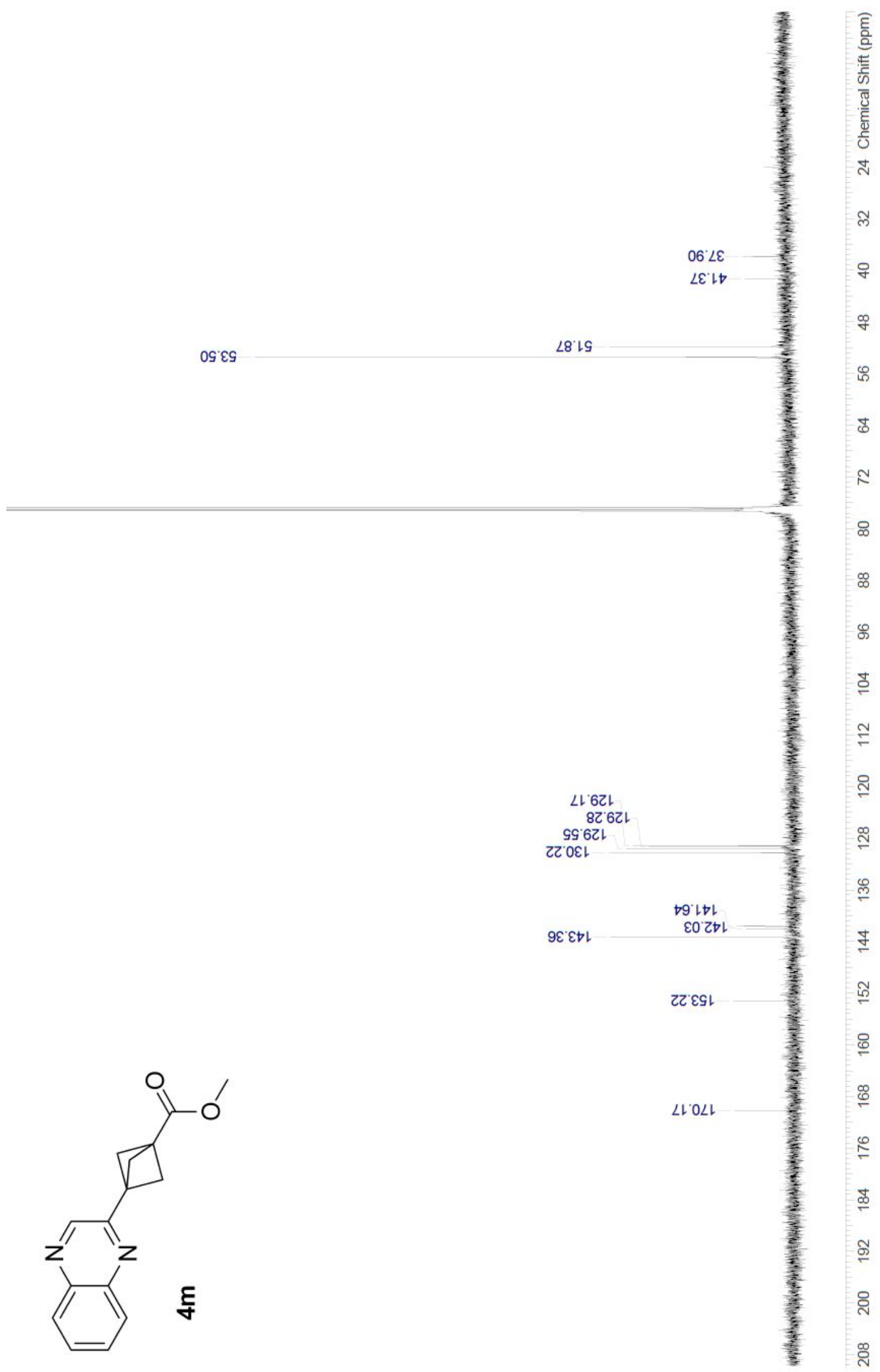


S53

182

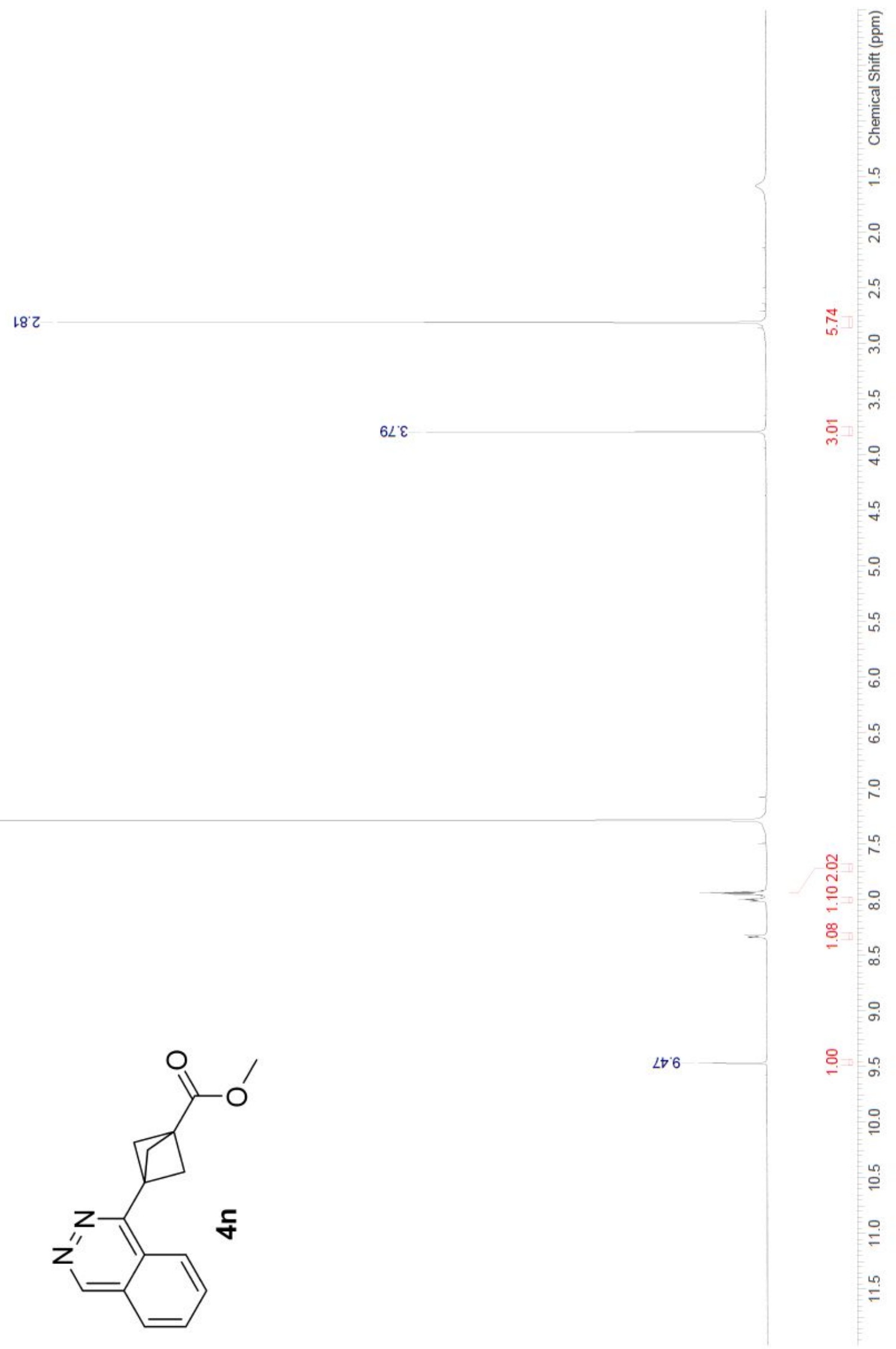




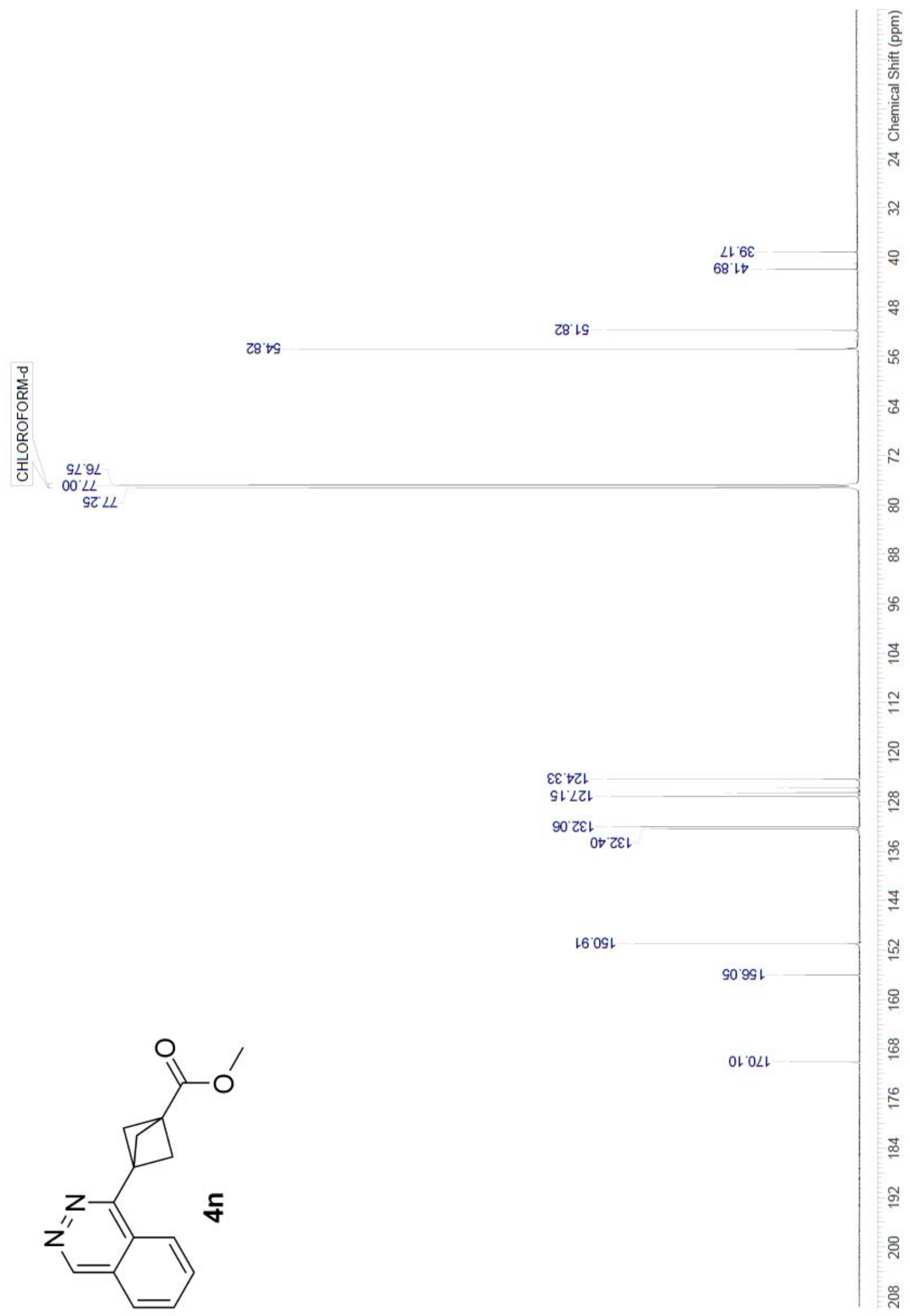


S55

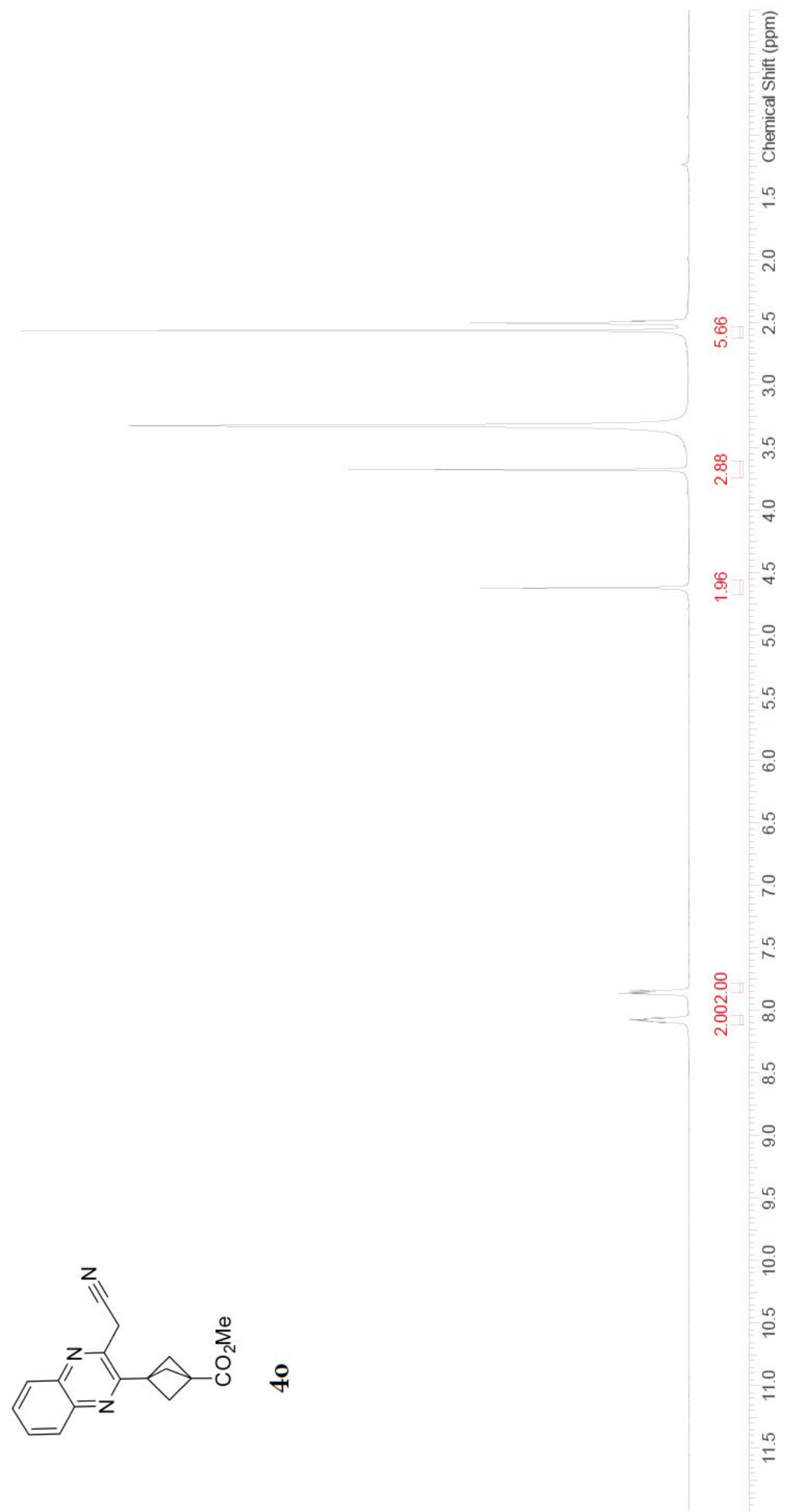


S56

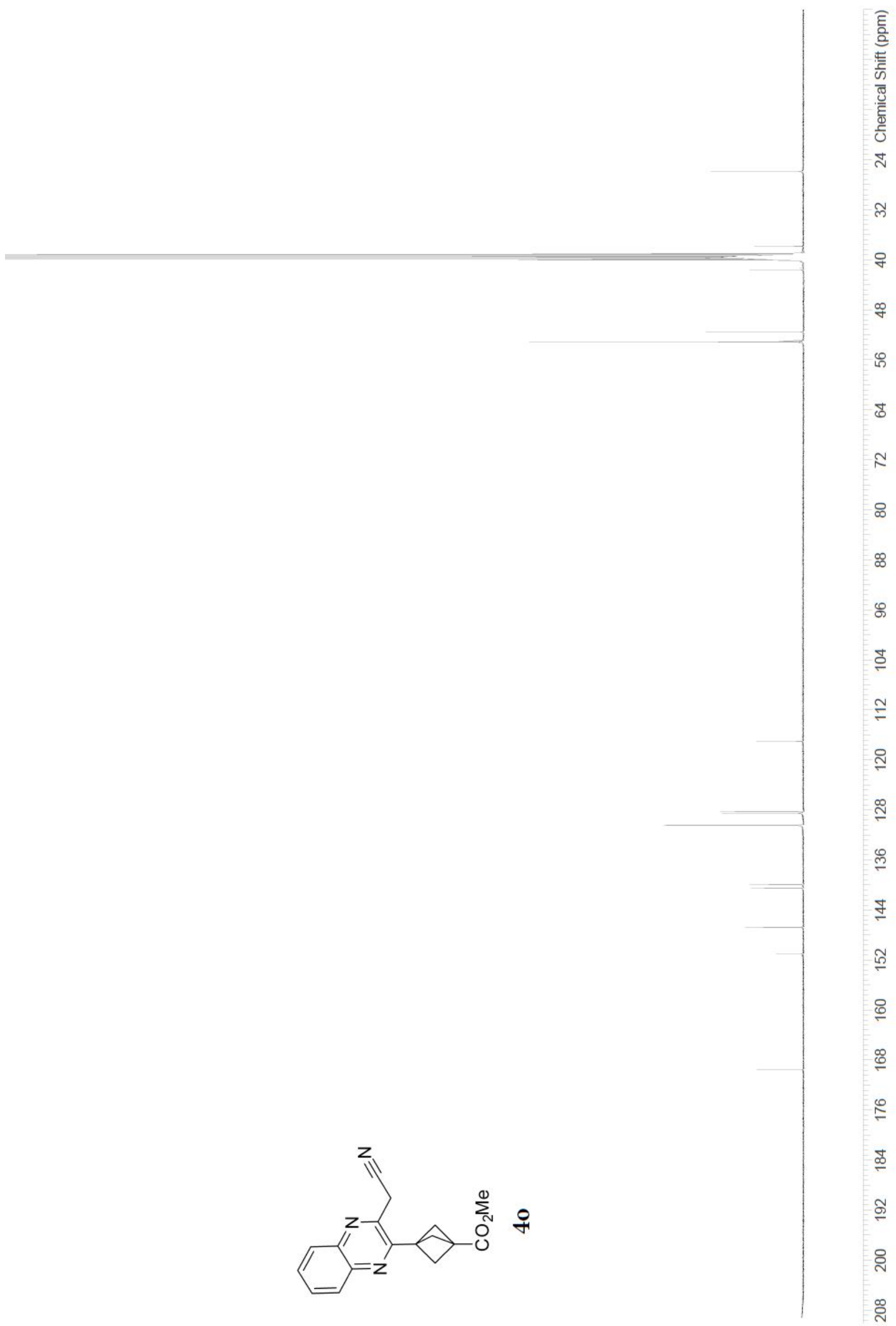




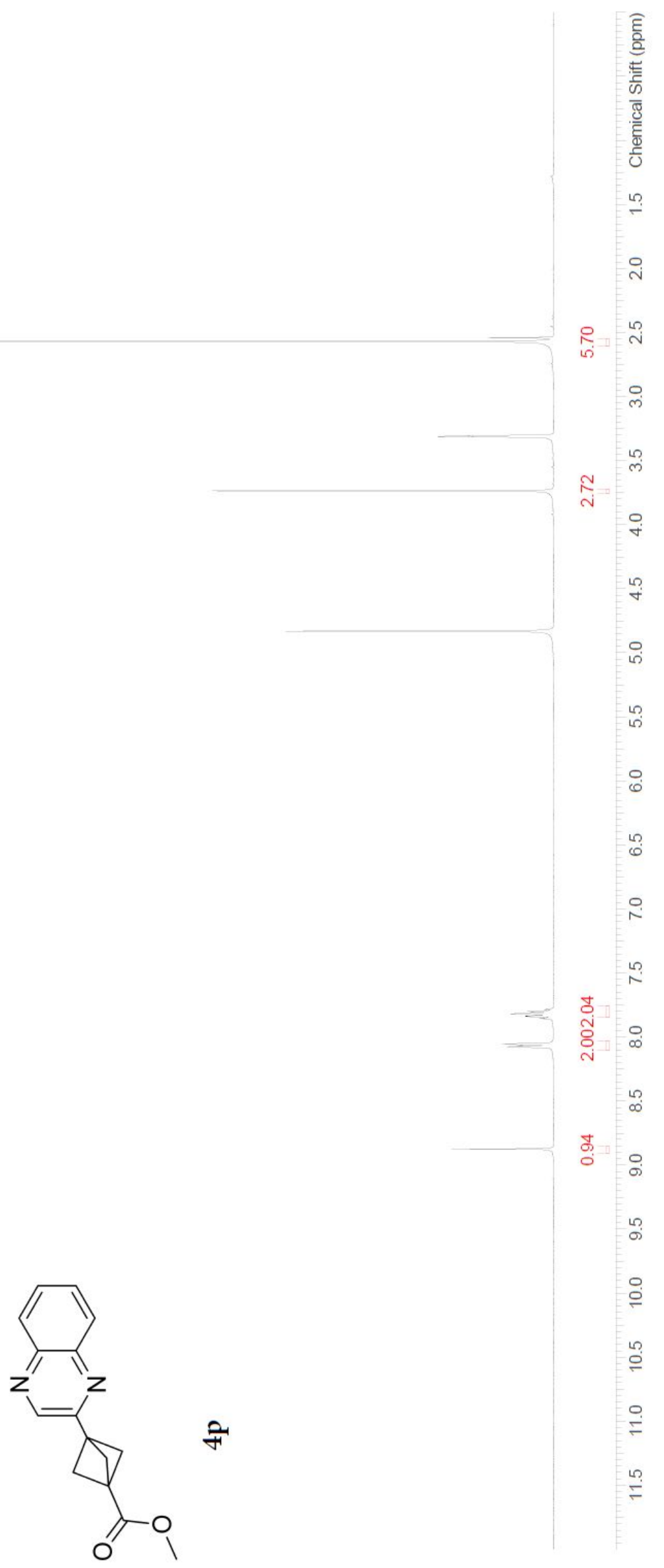


S58

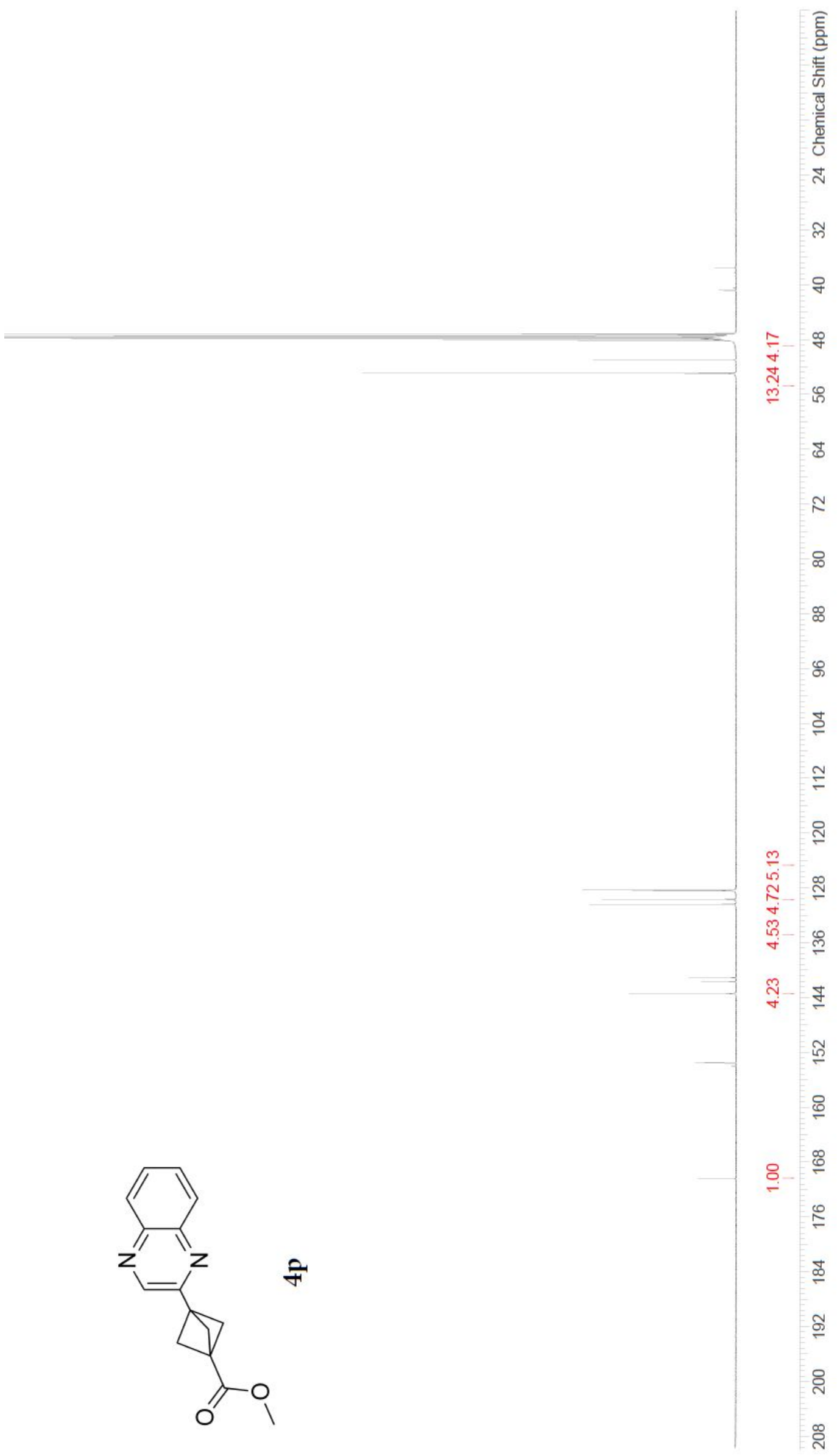




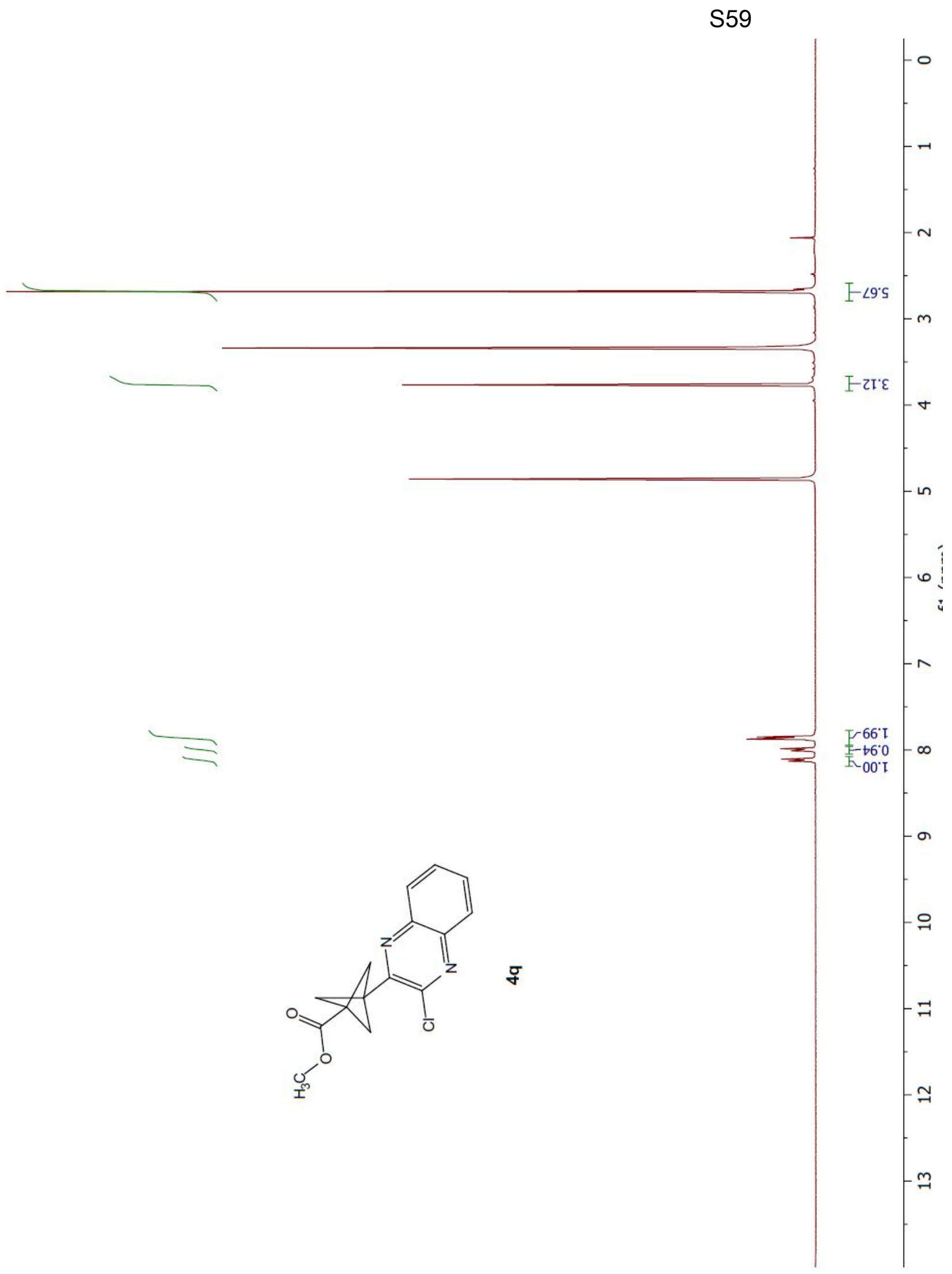




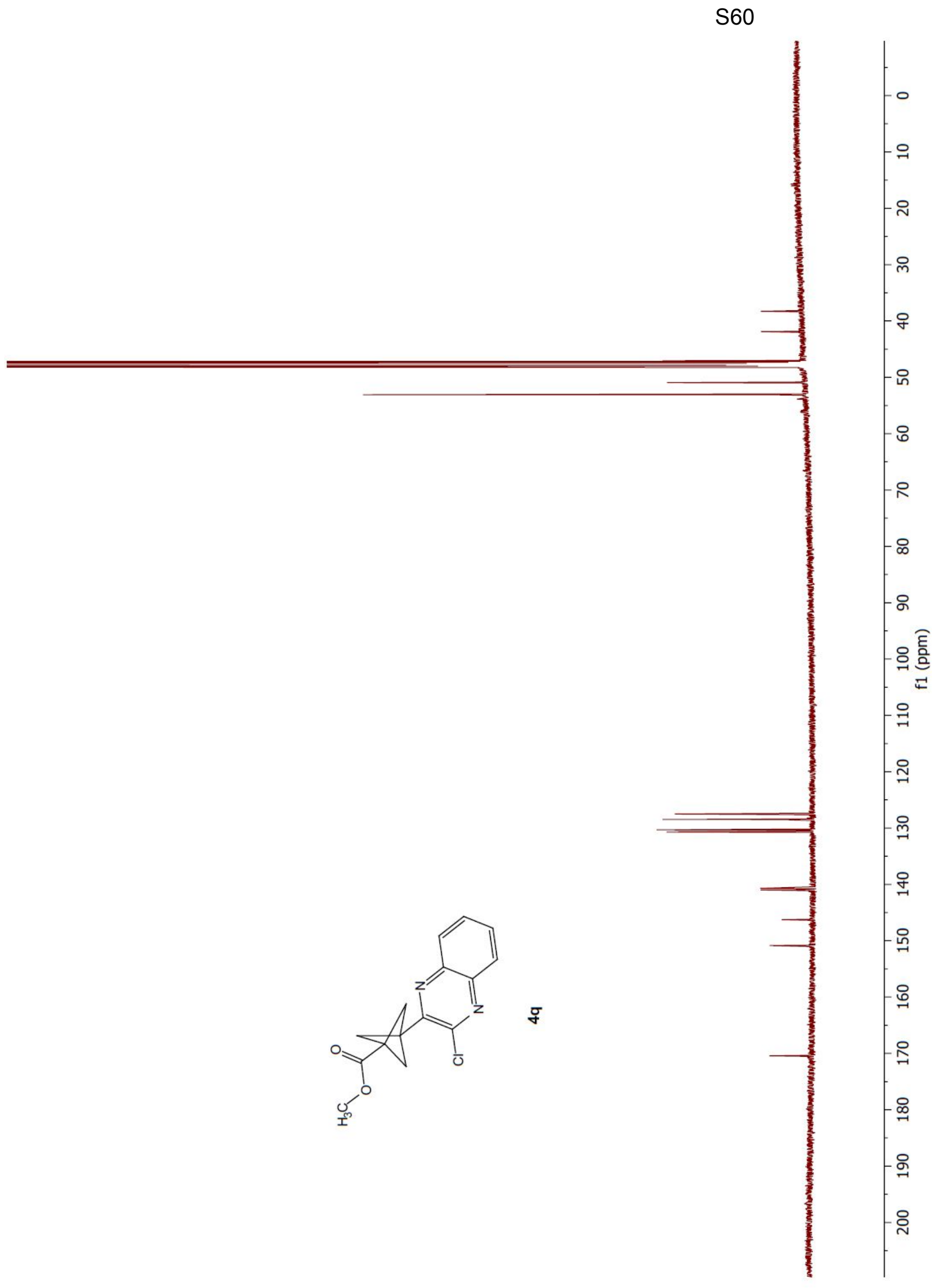




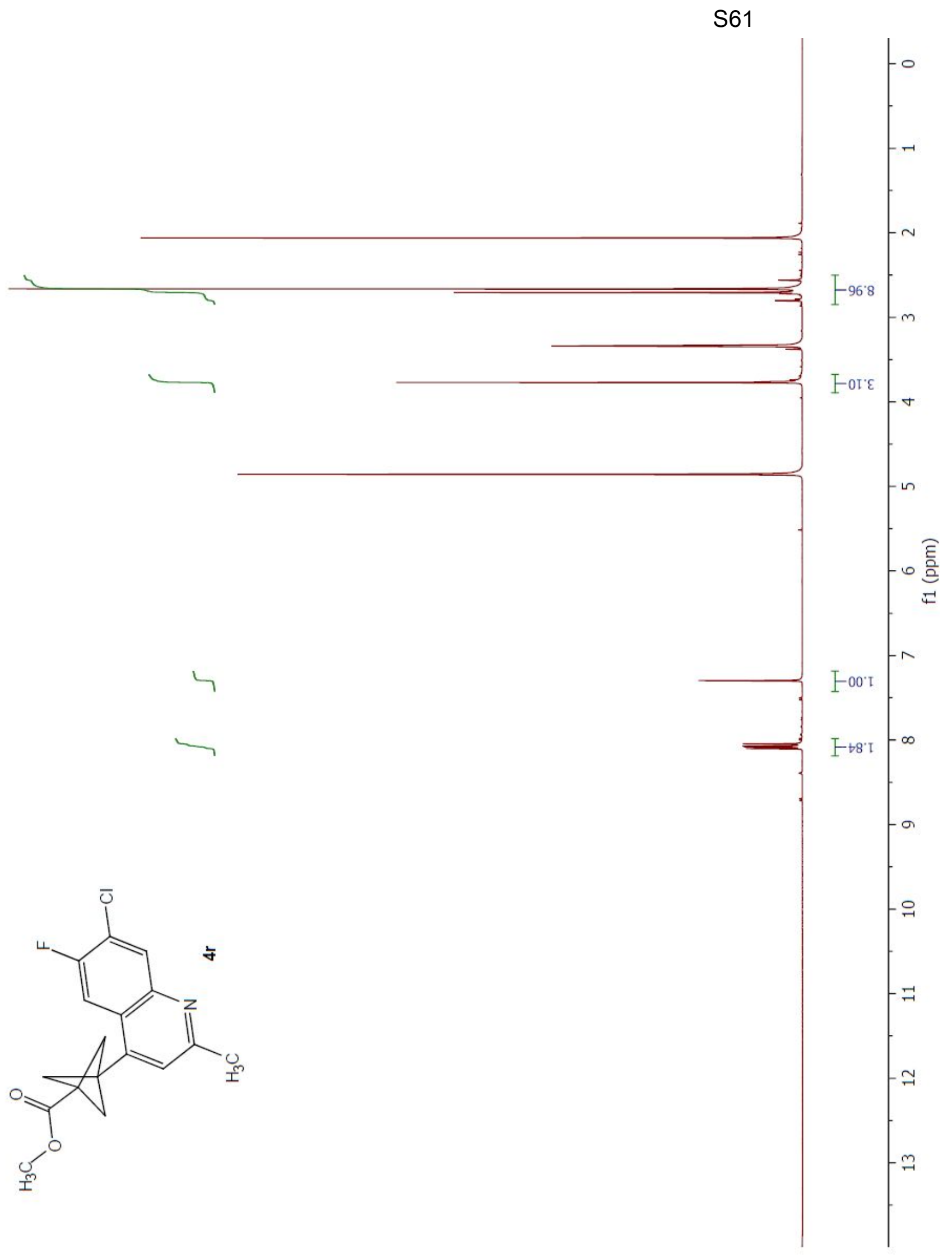




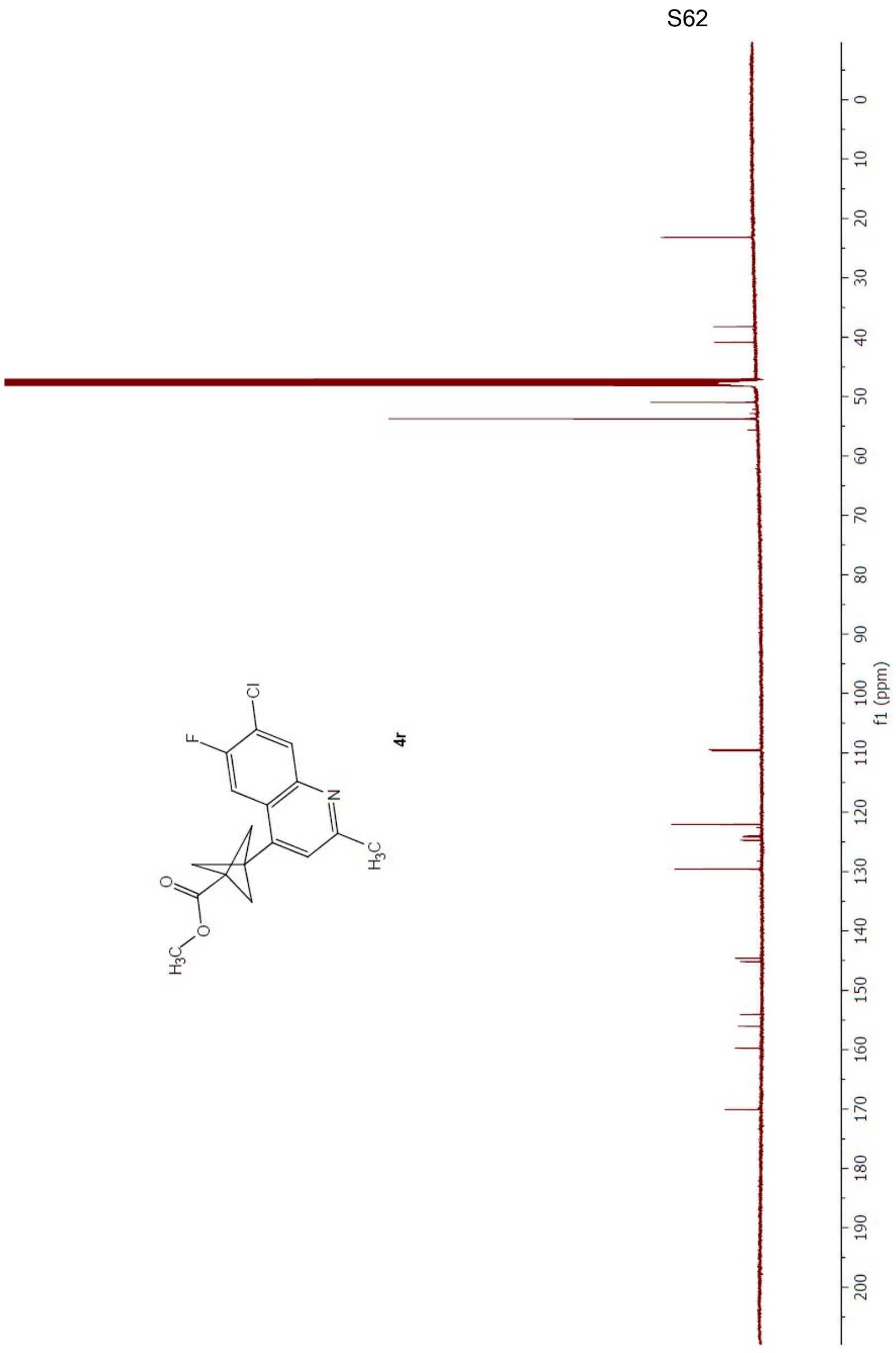




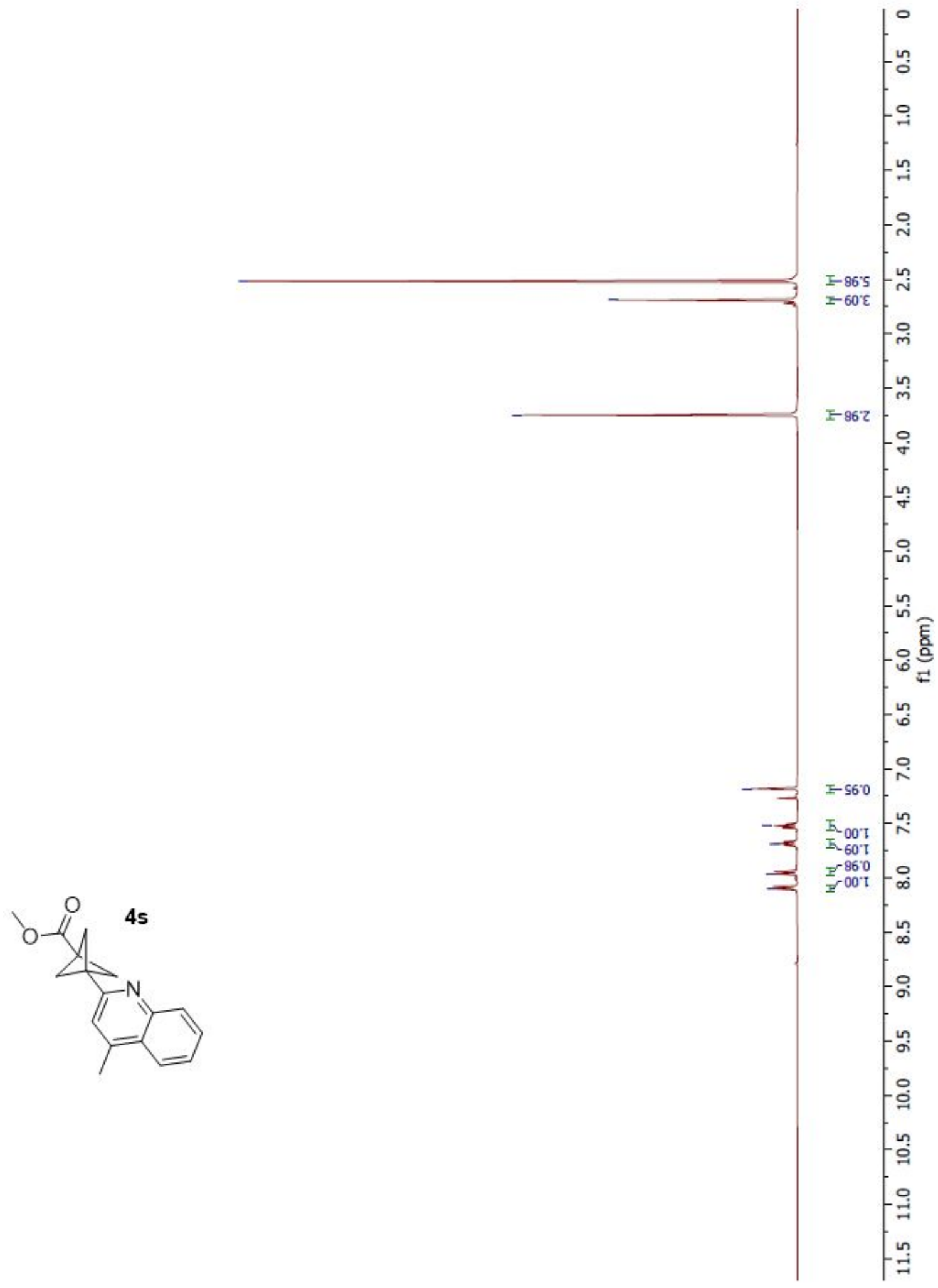




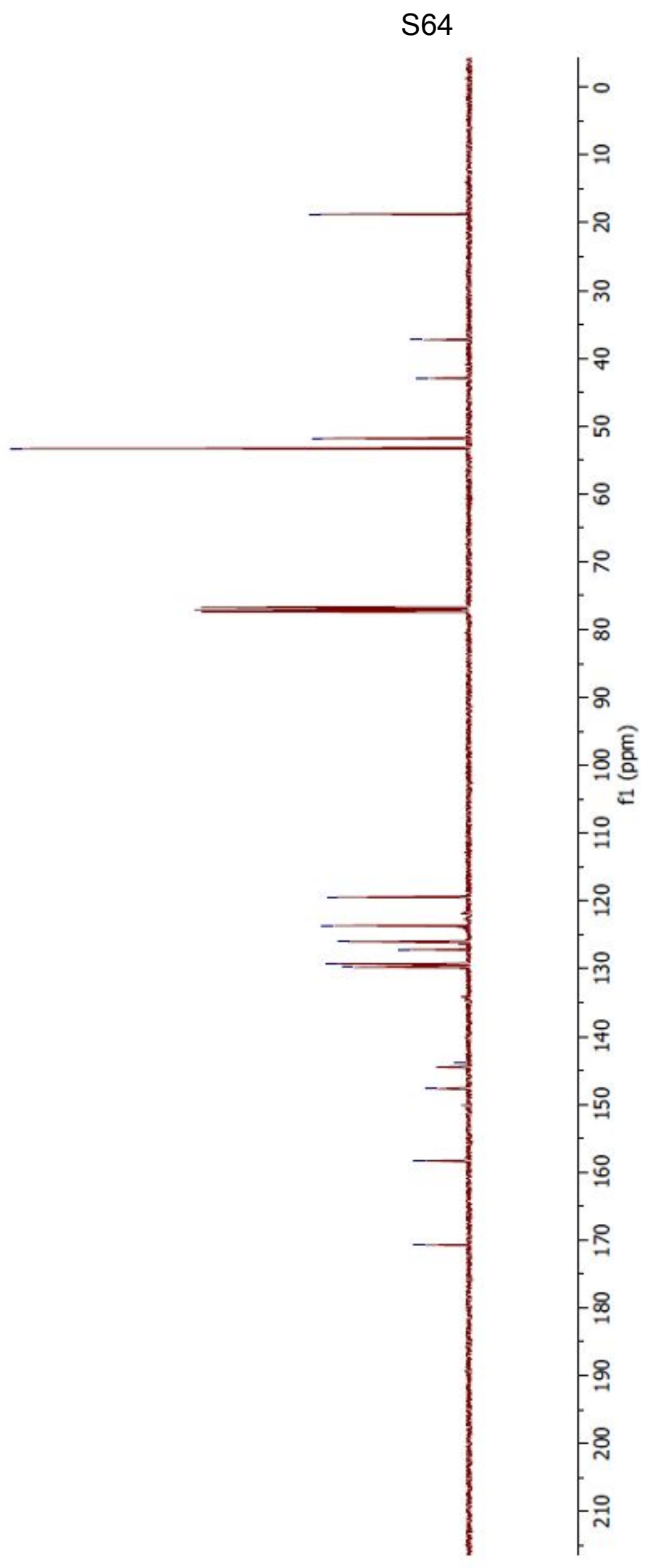


S65
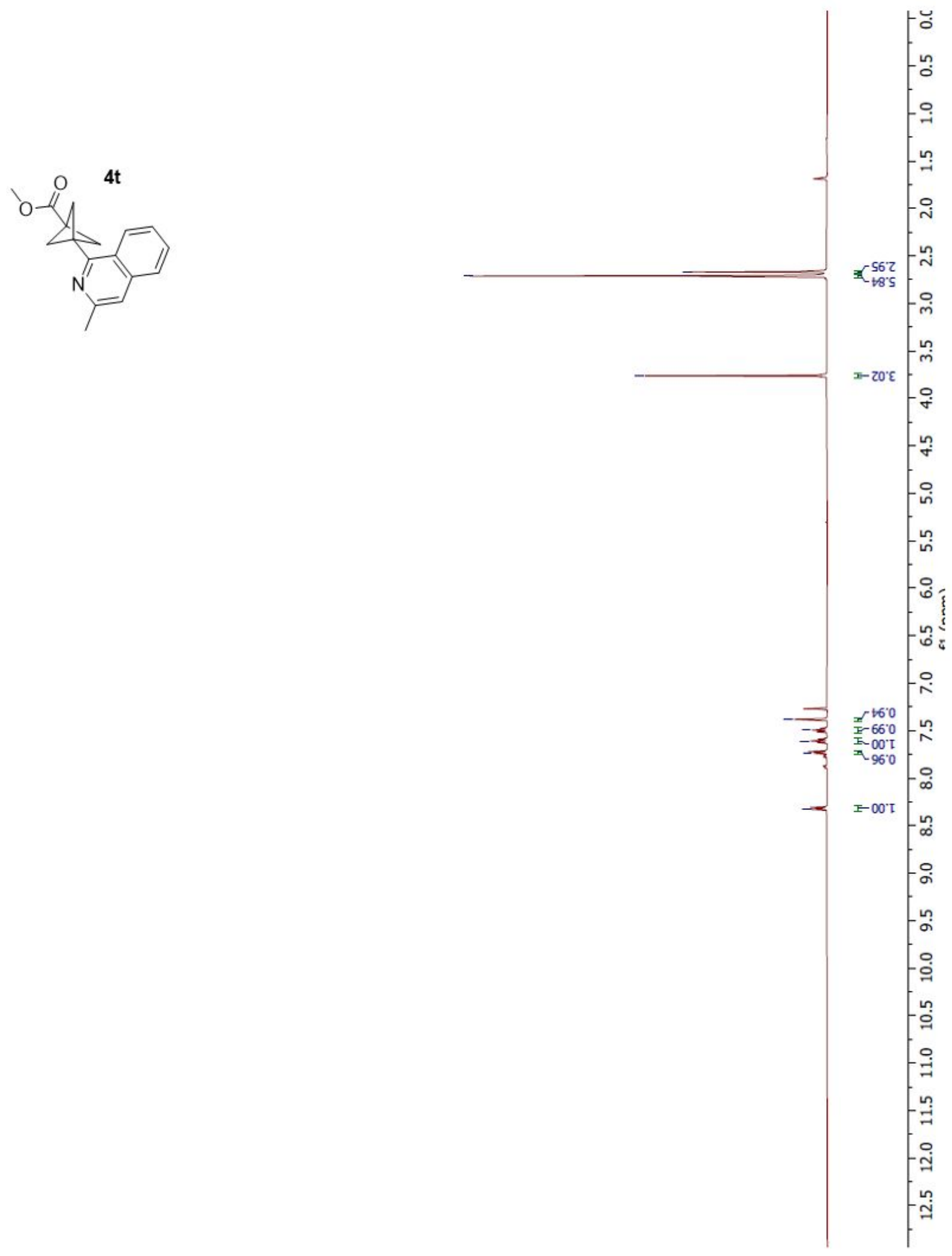


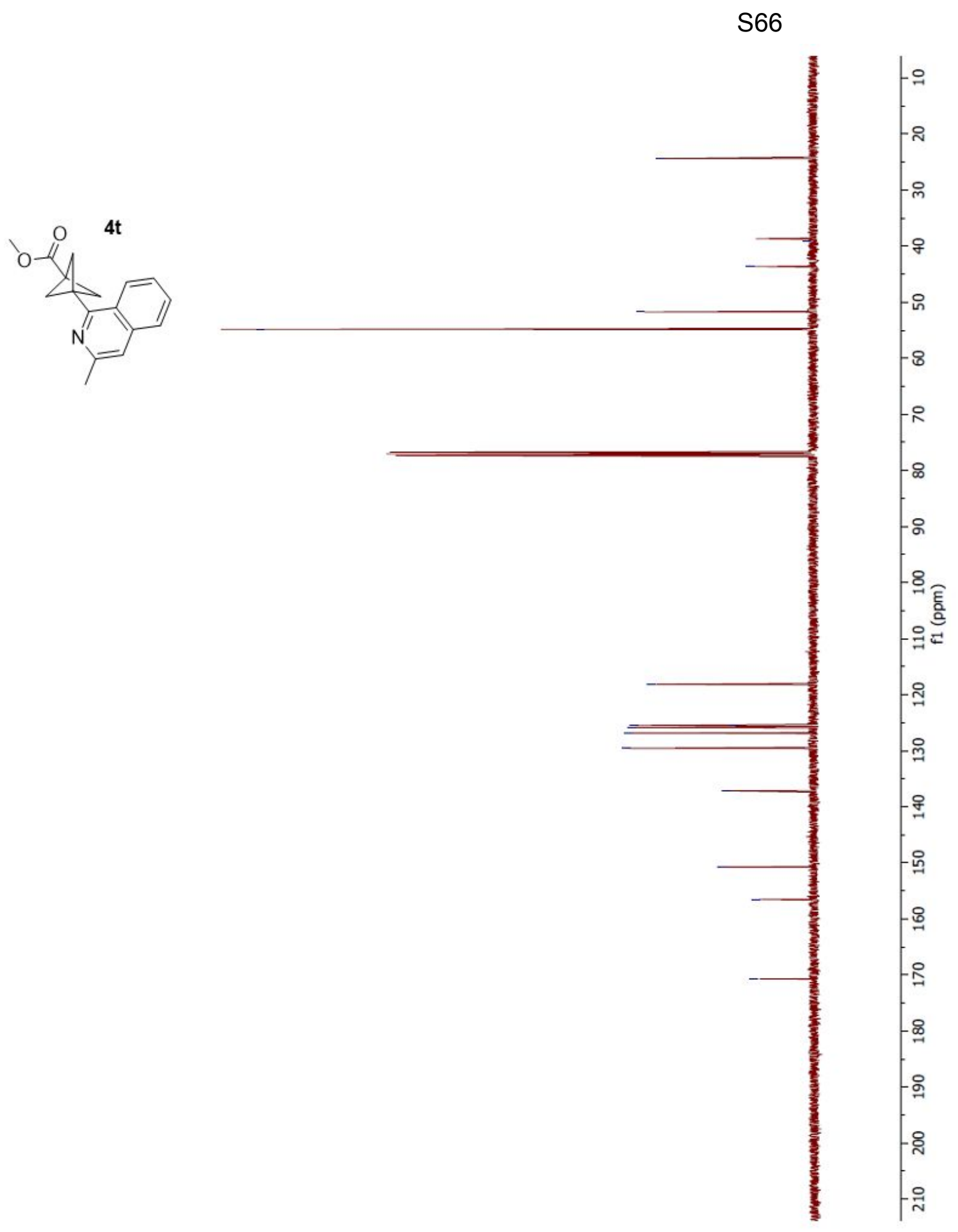




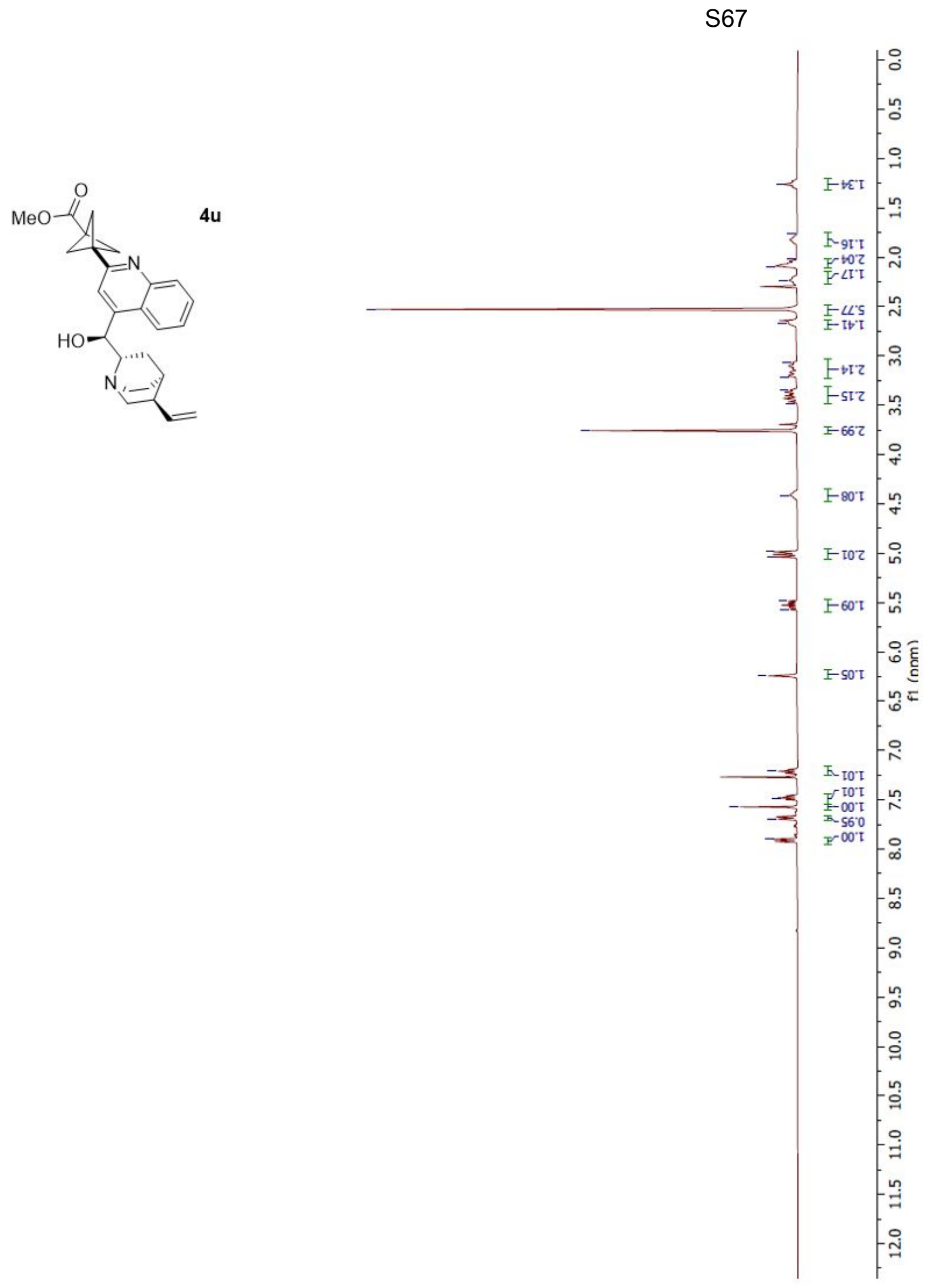




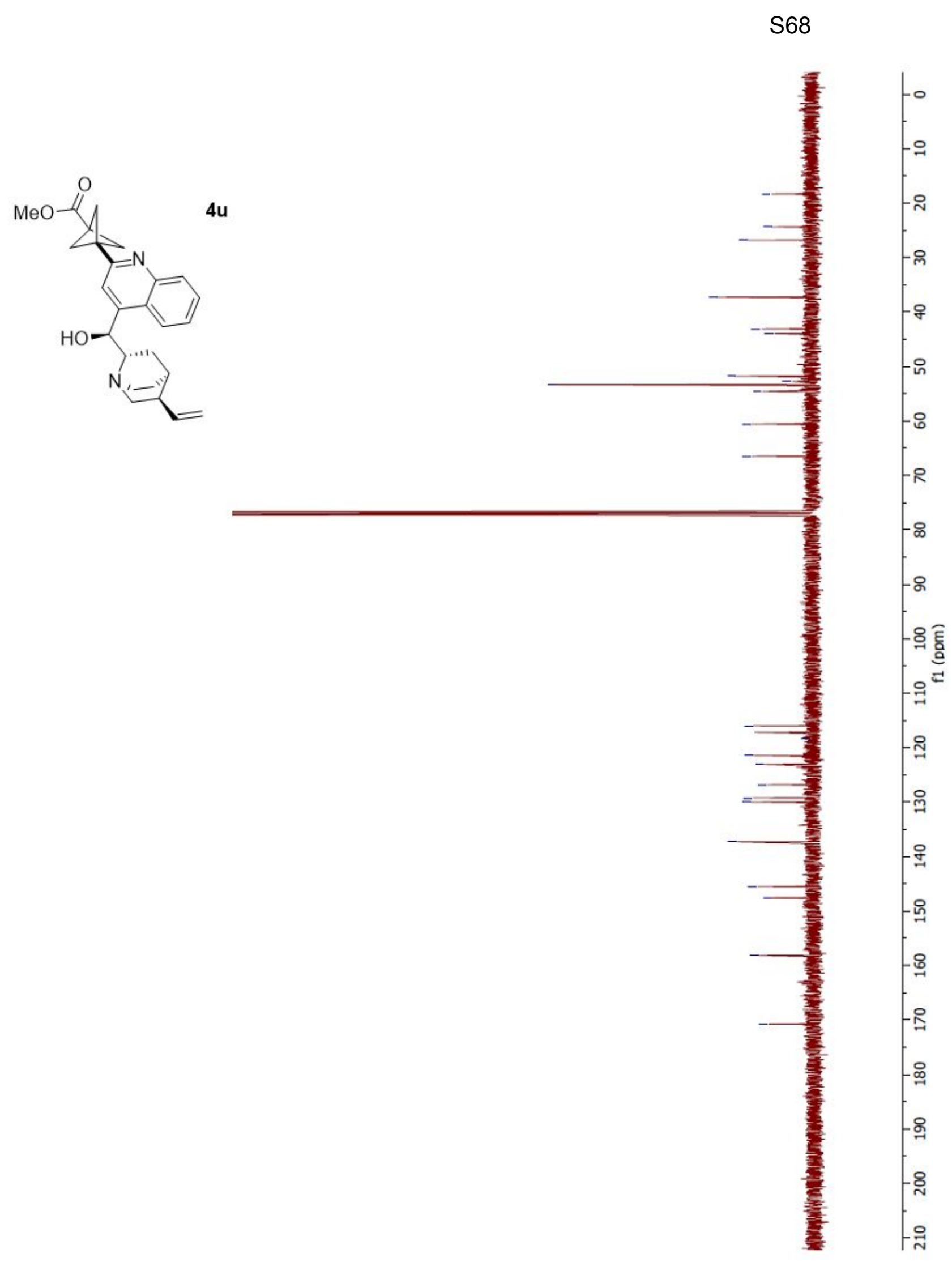




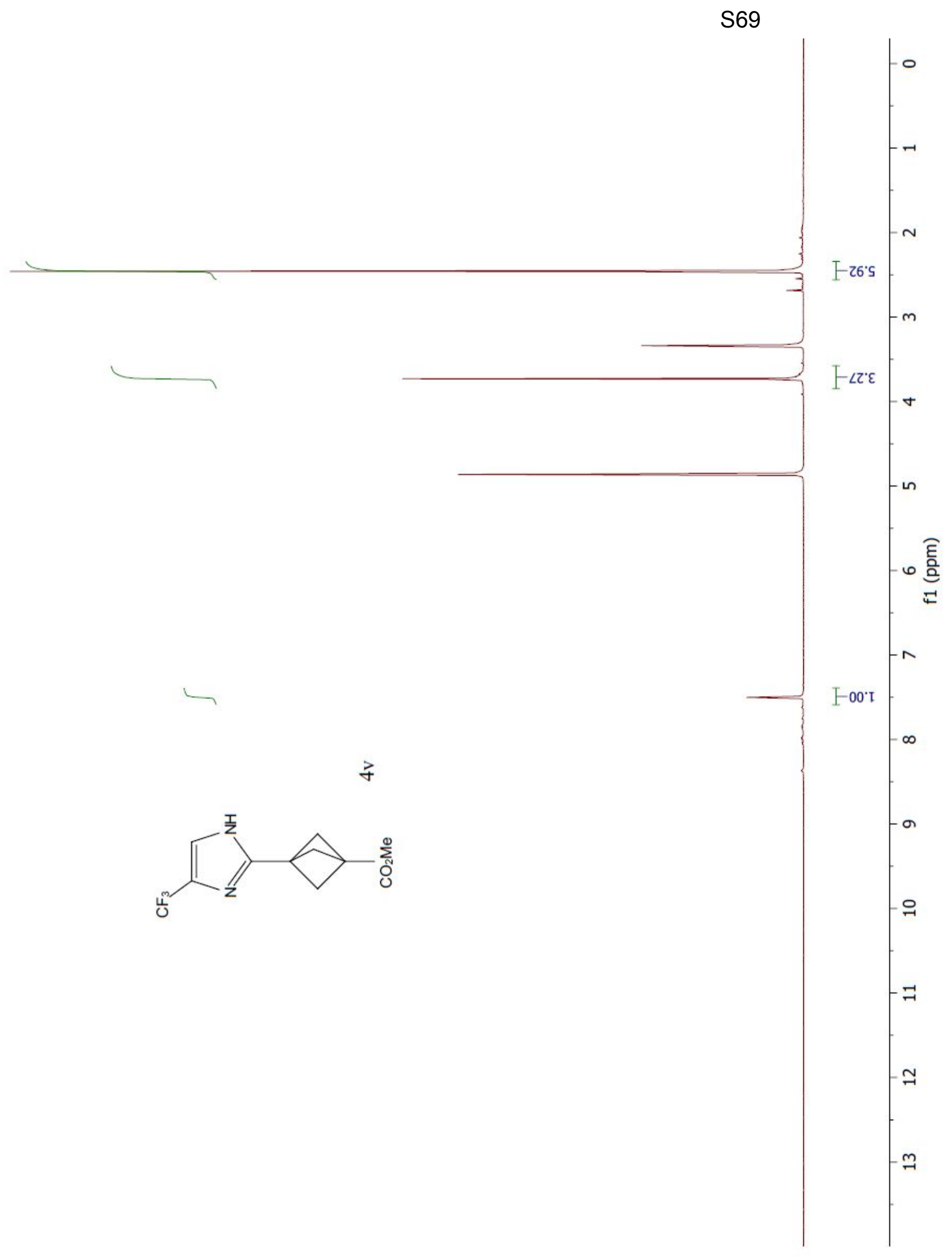




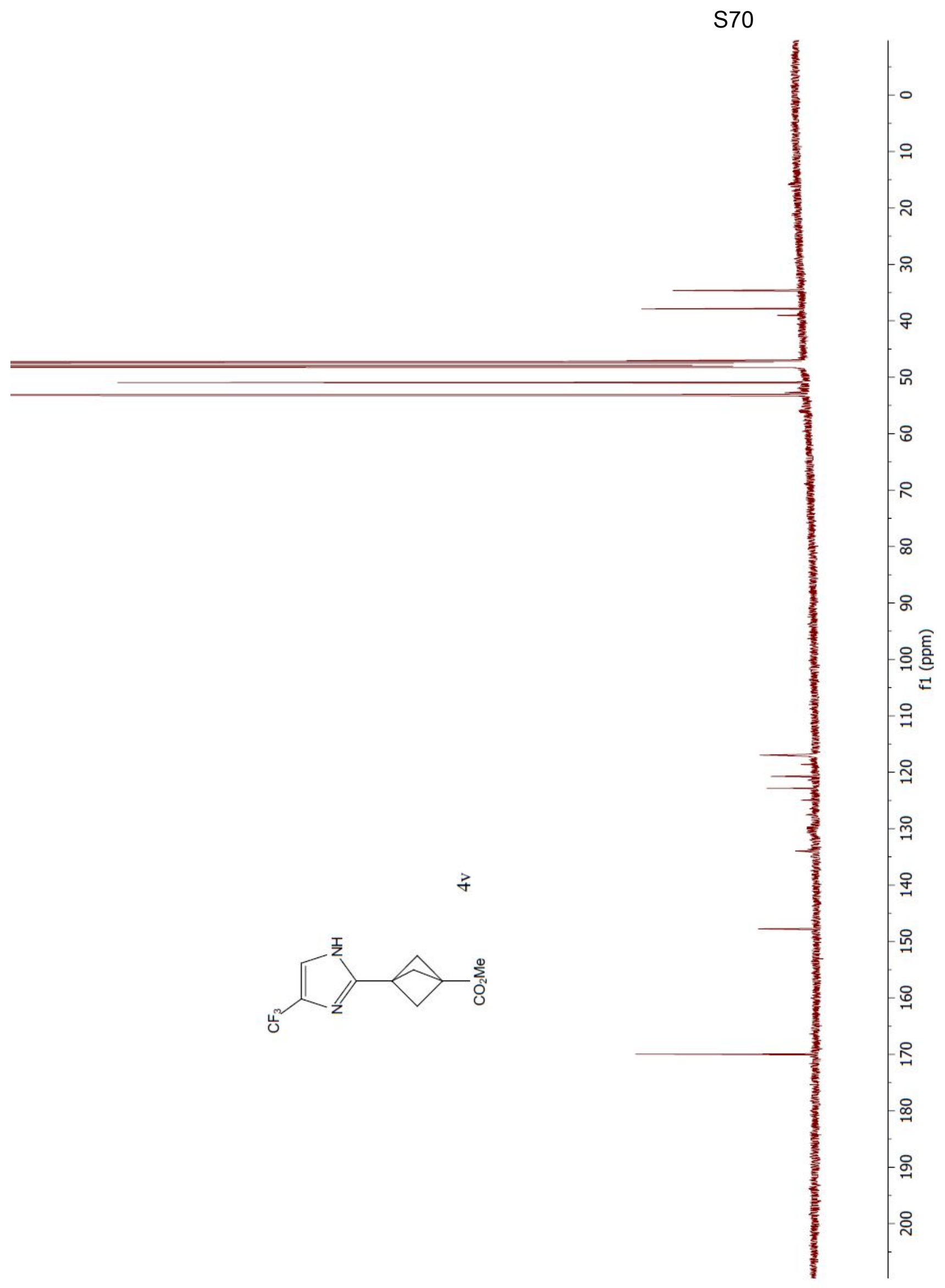




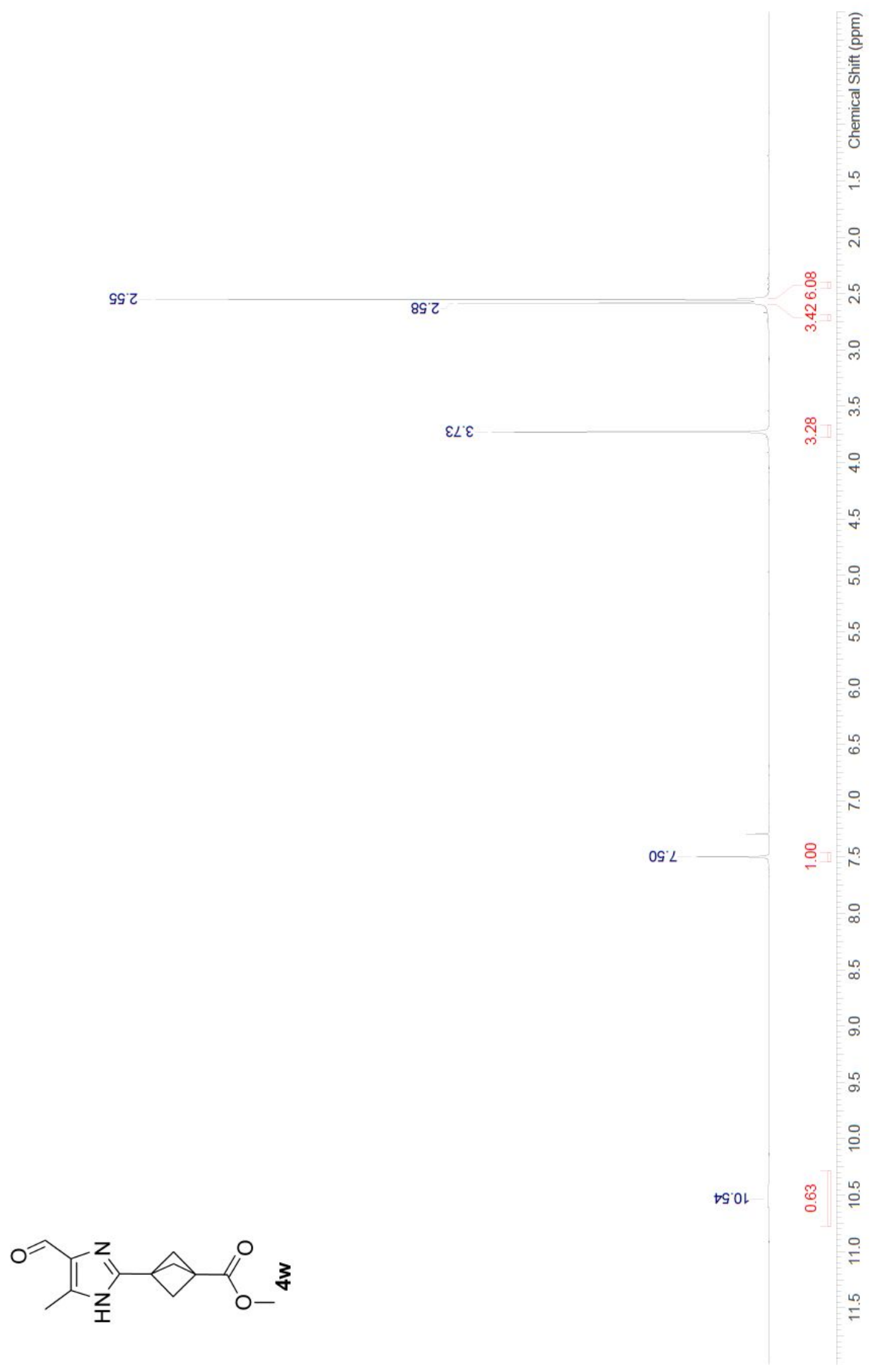




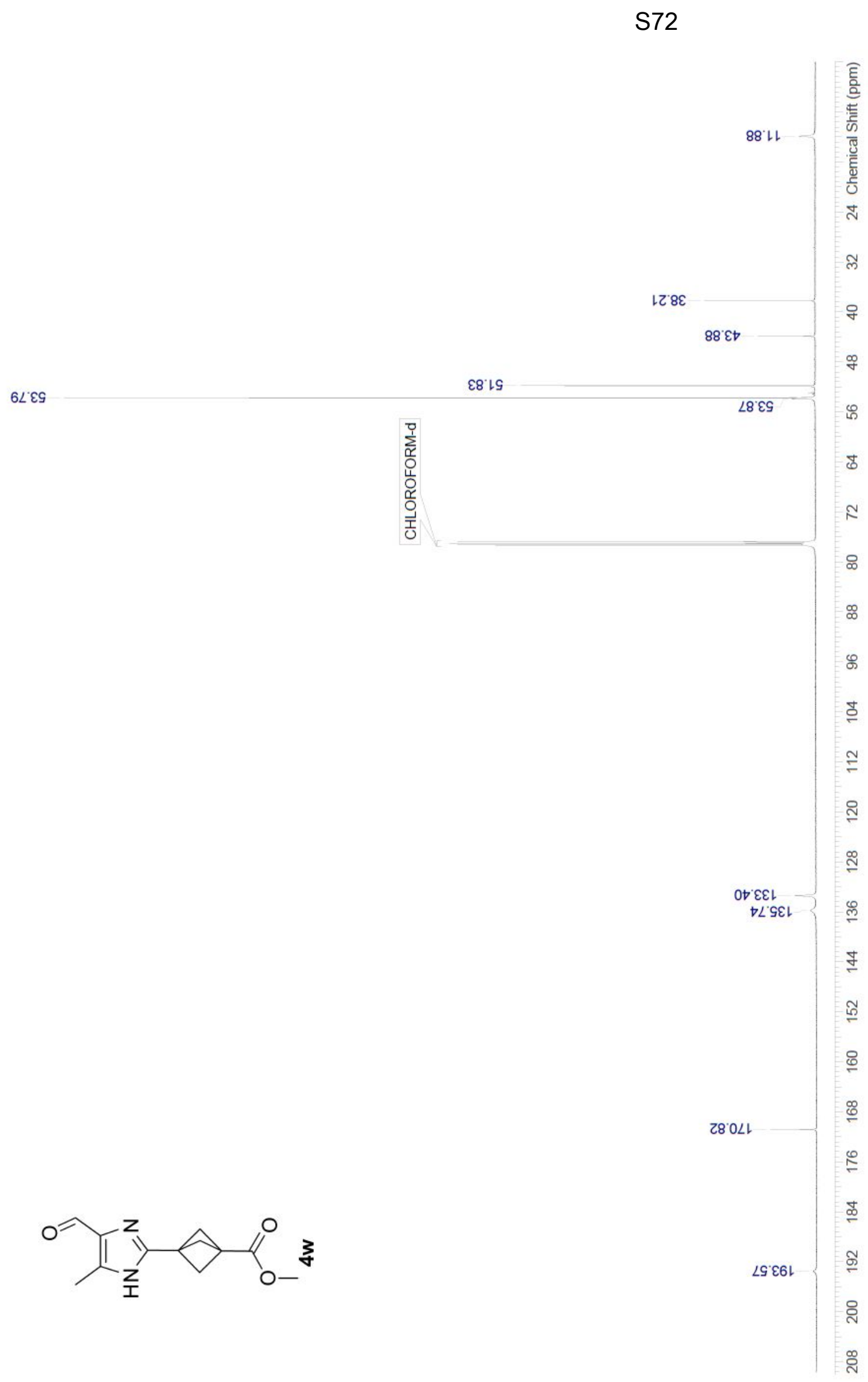




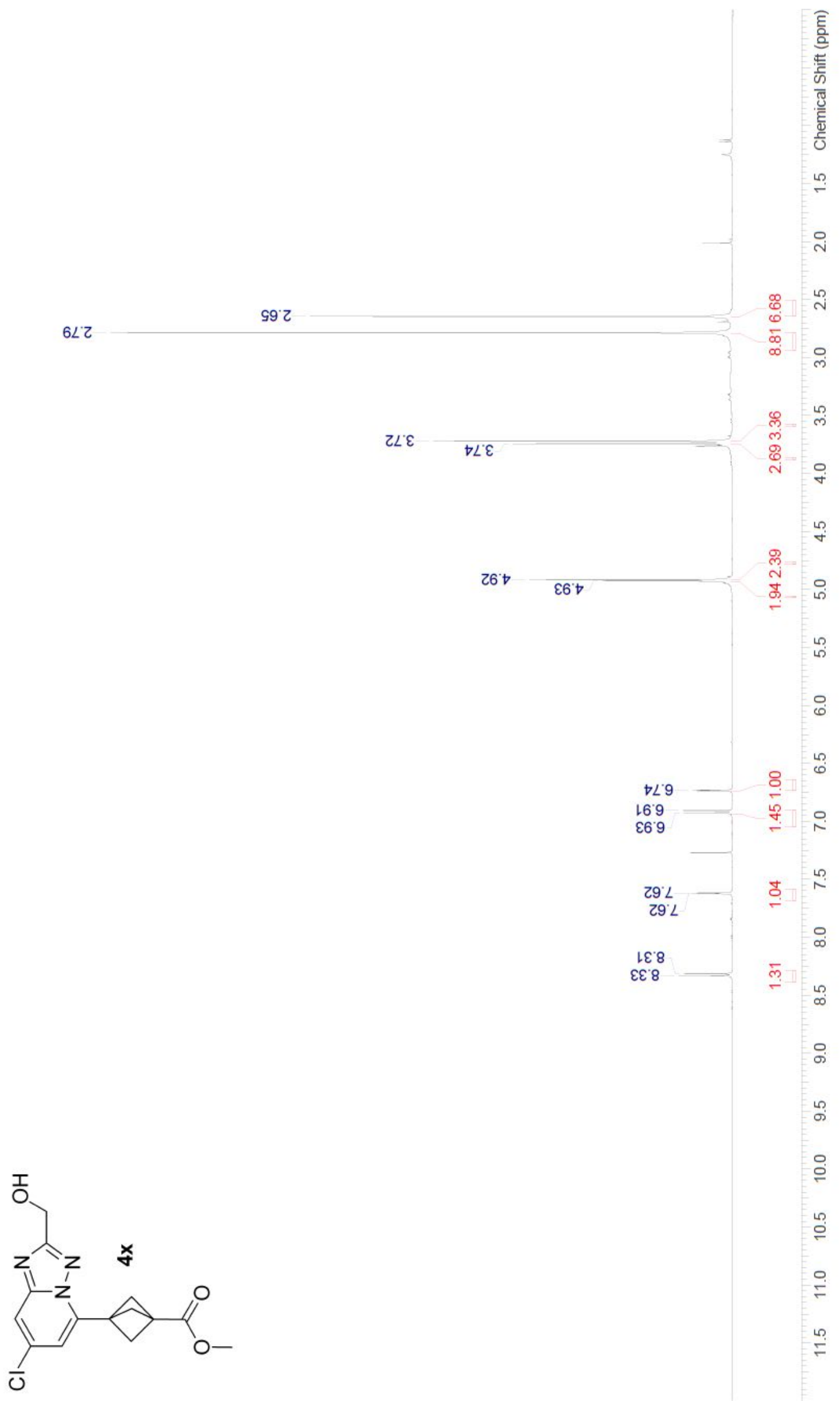




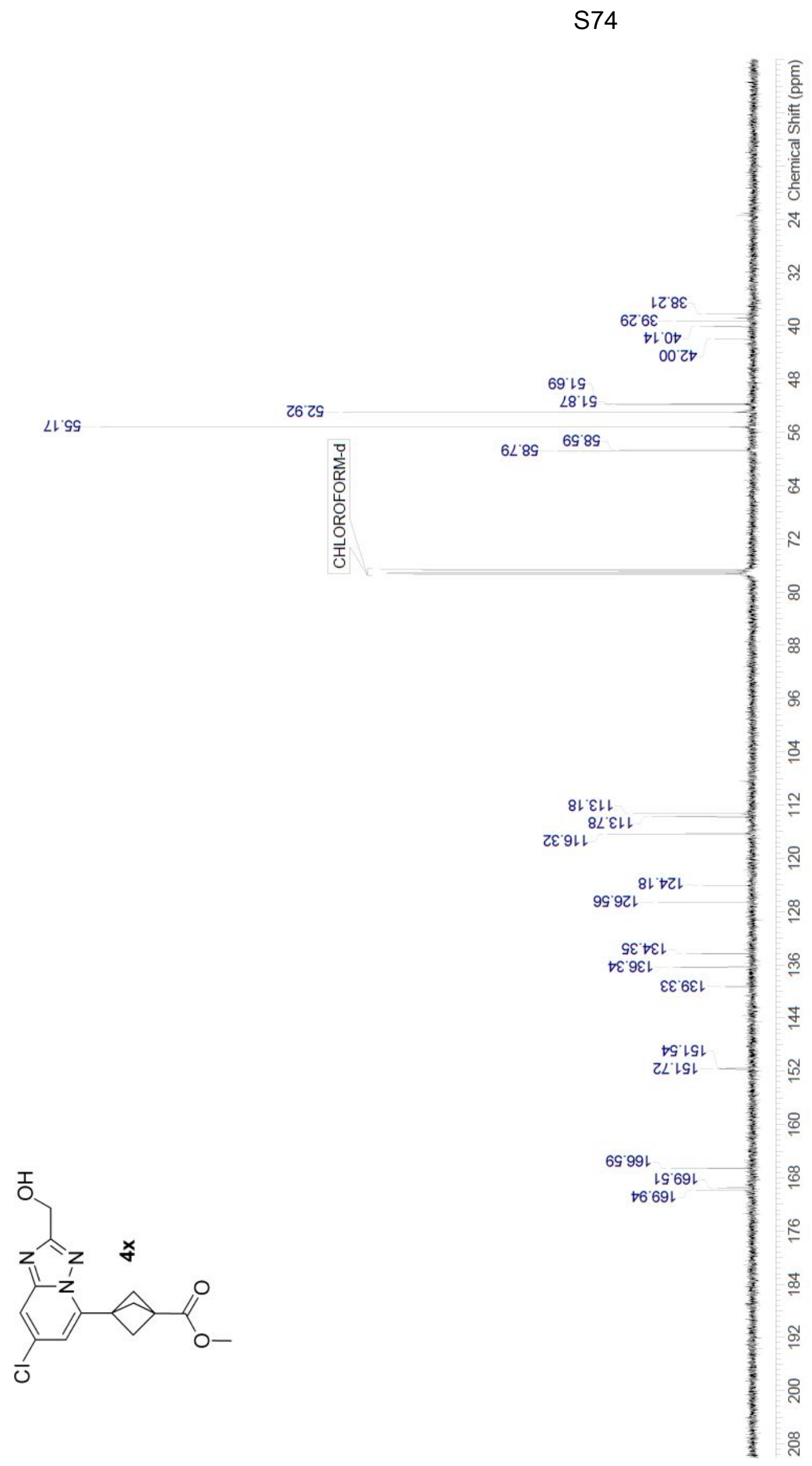




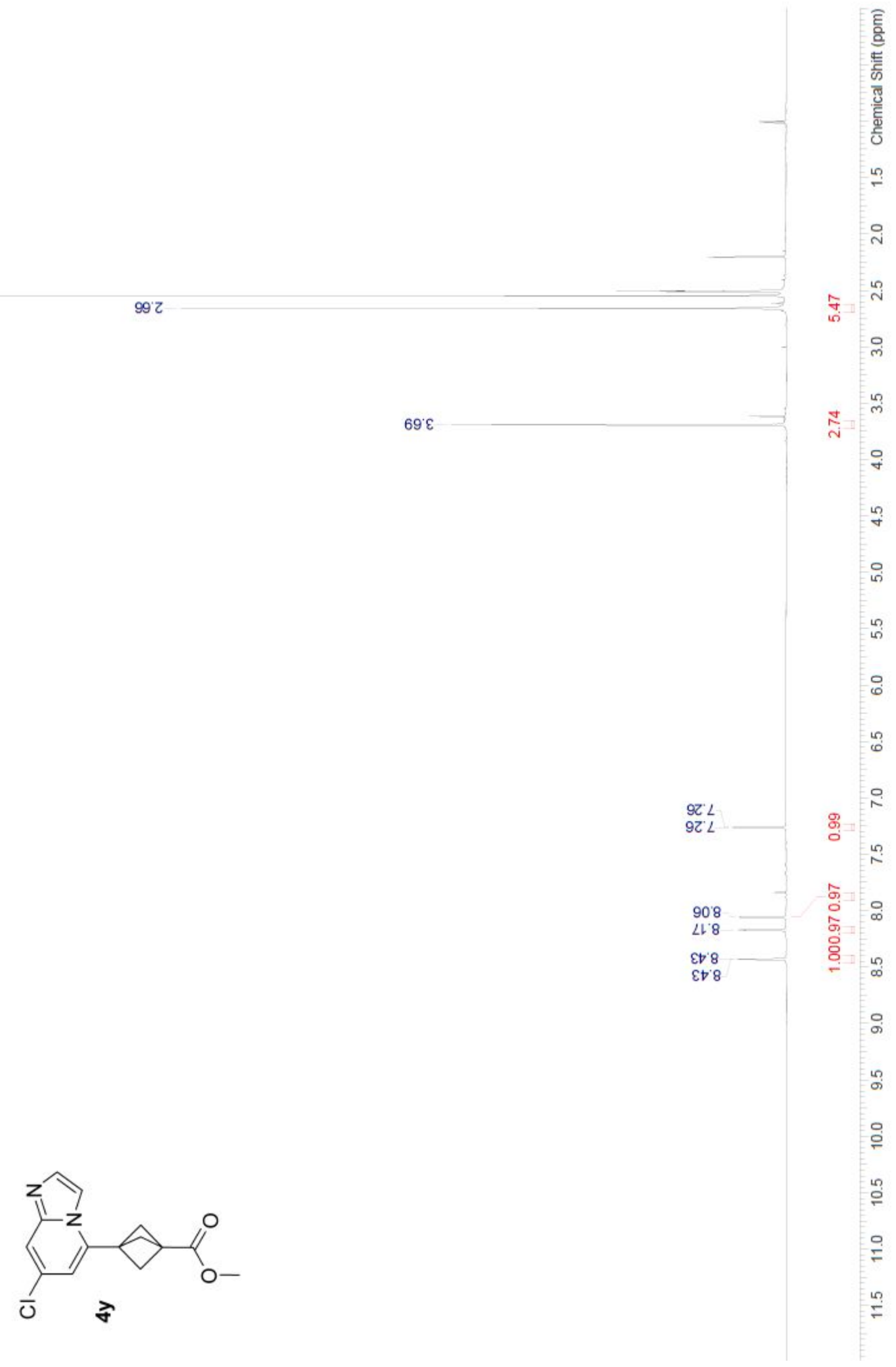




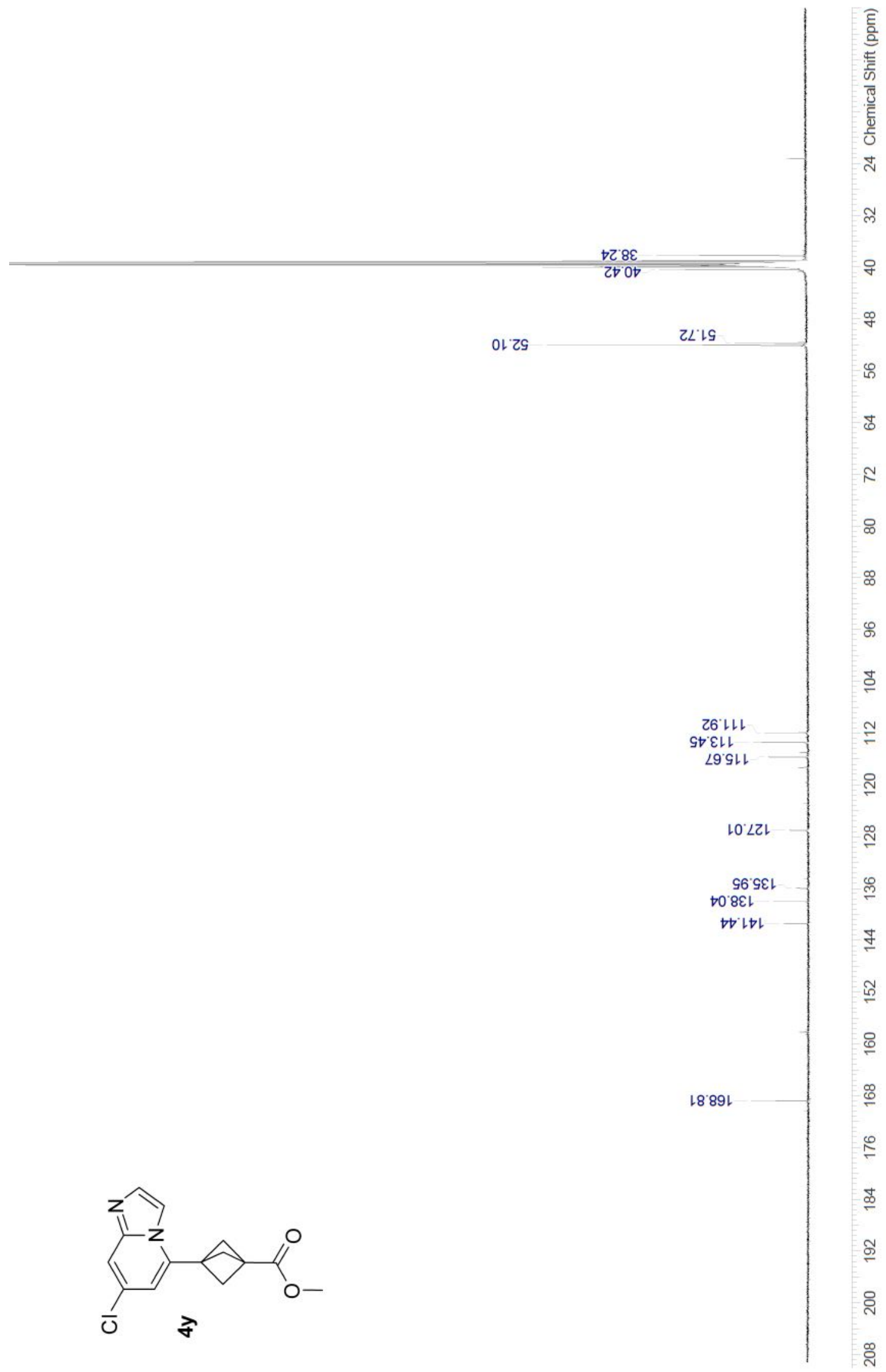




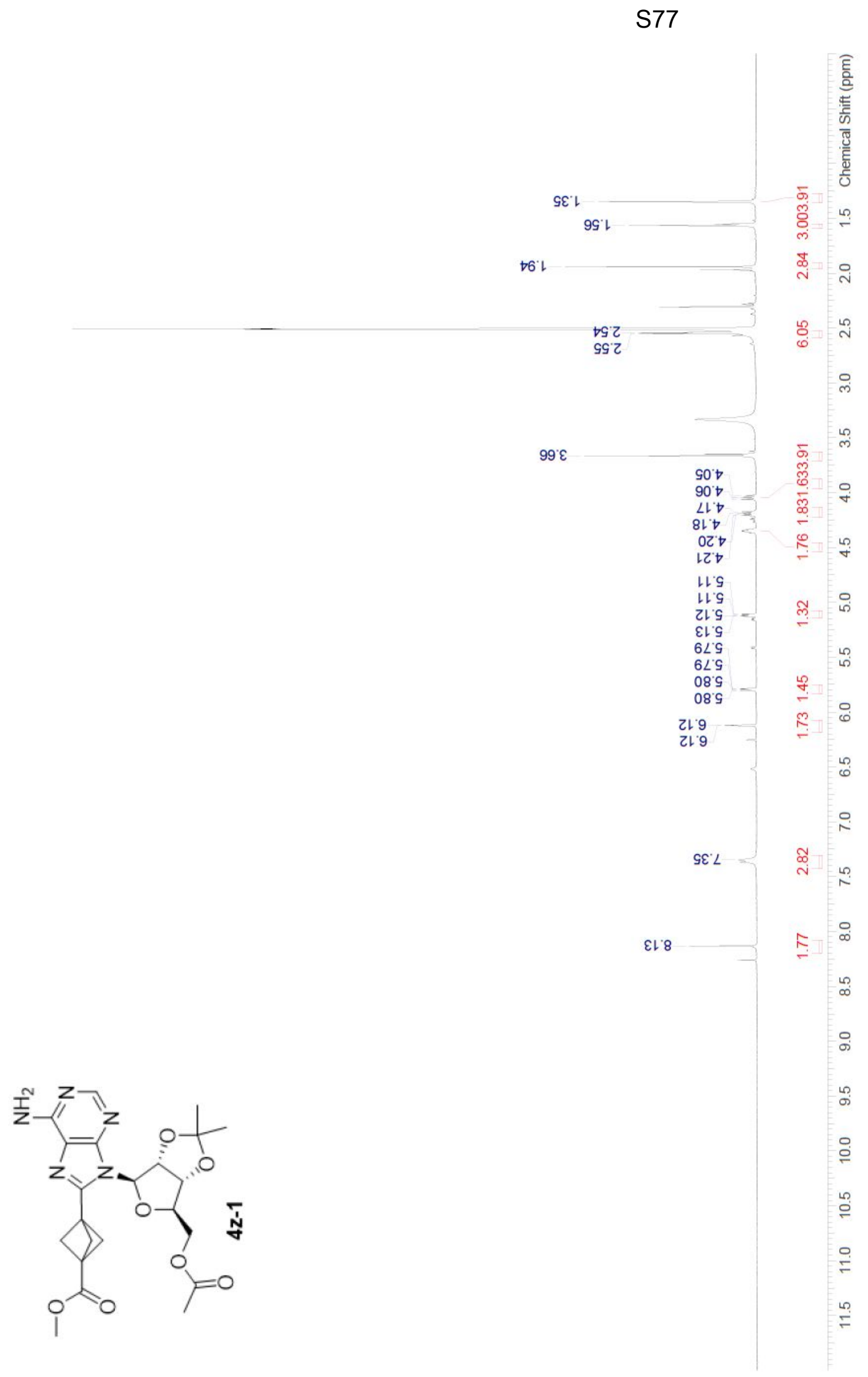




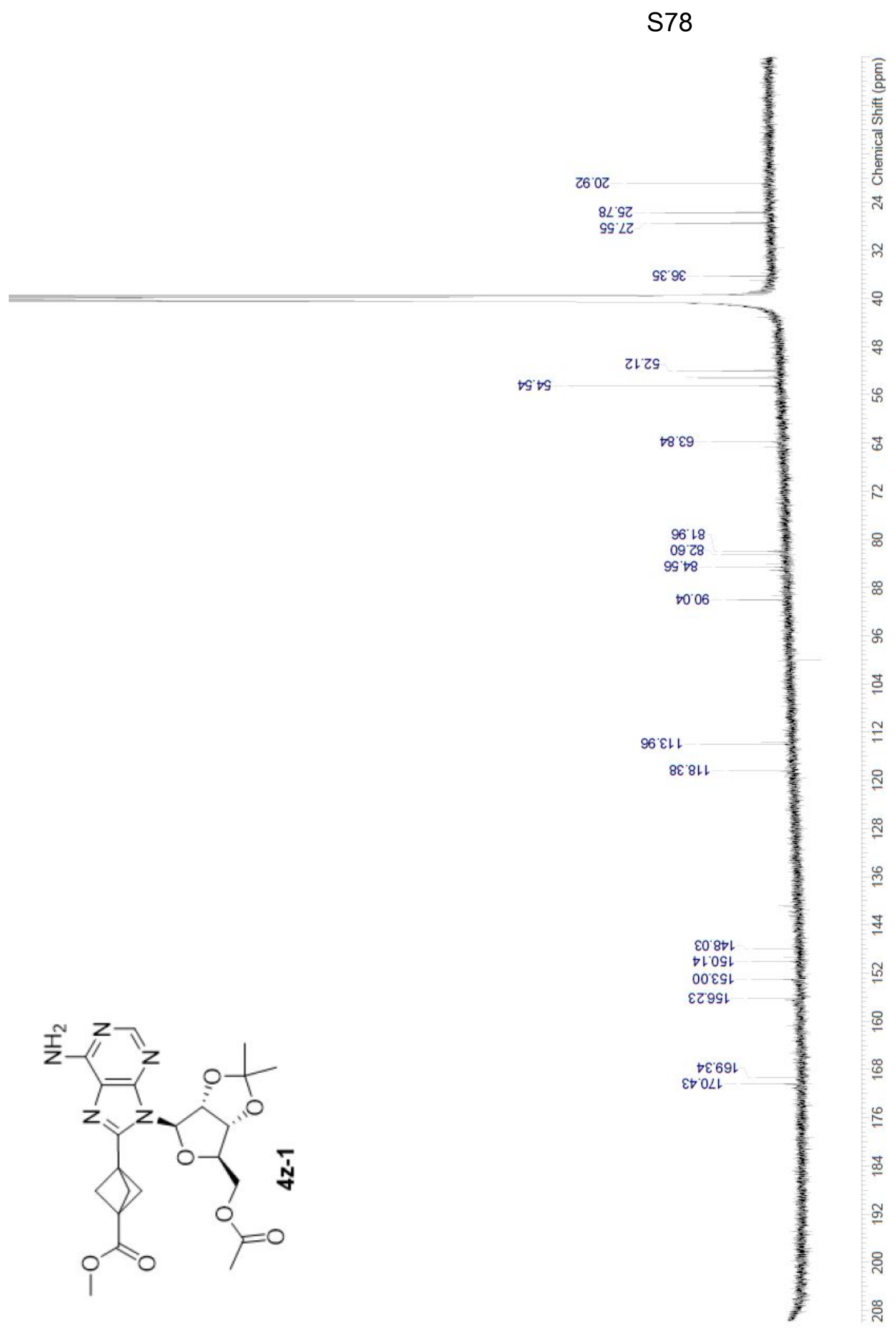




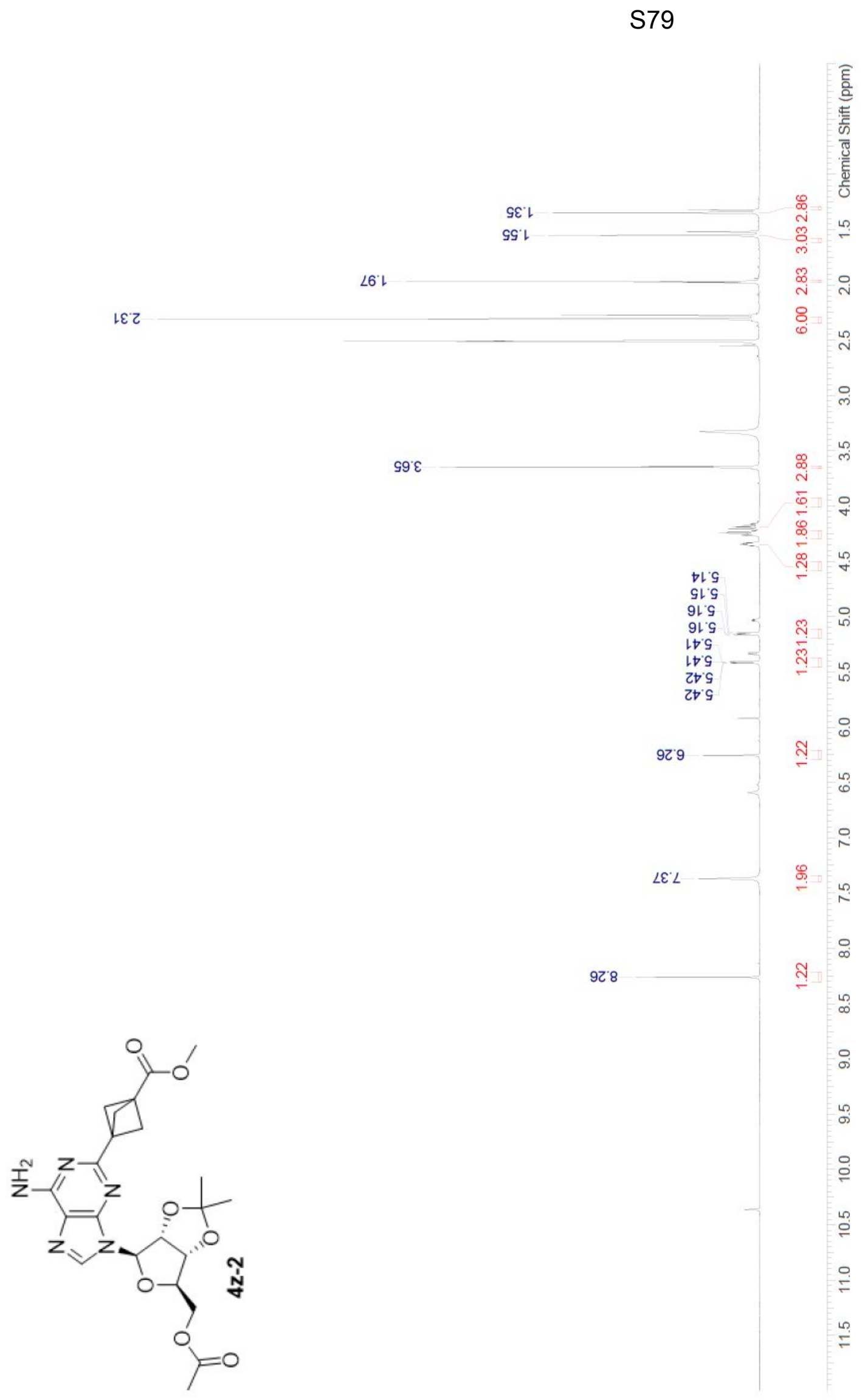




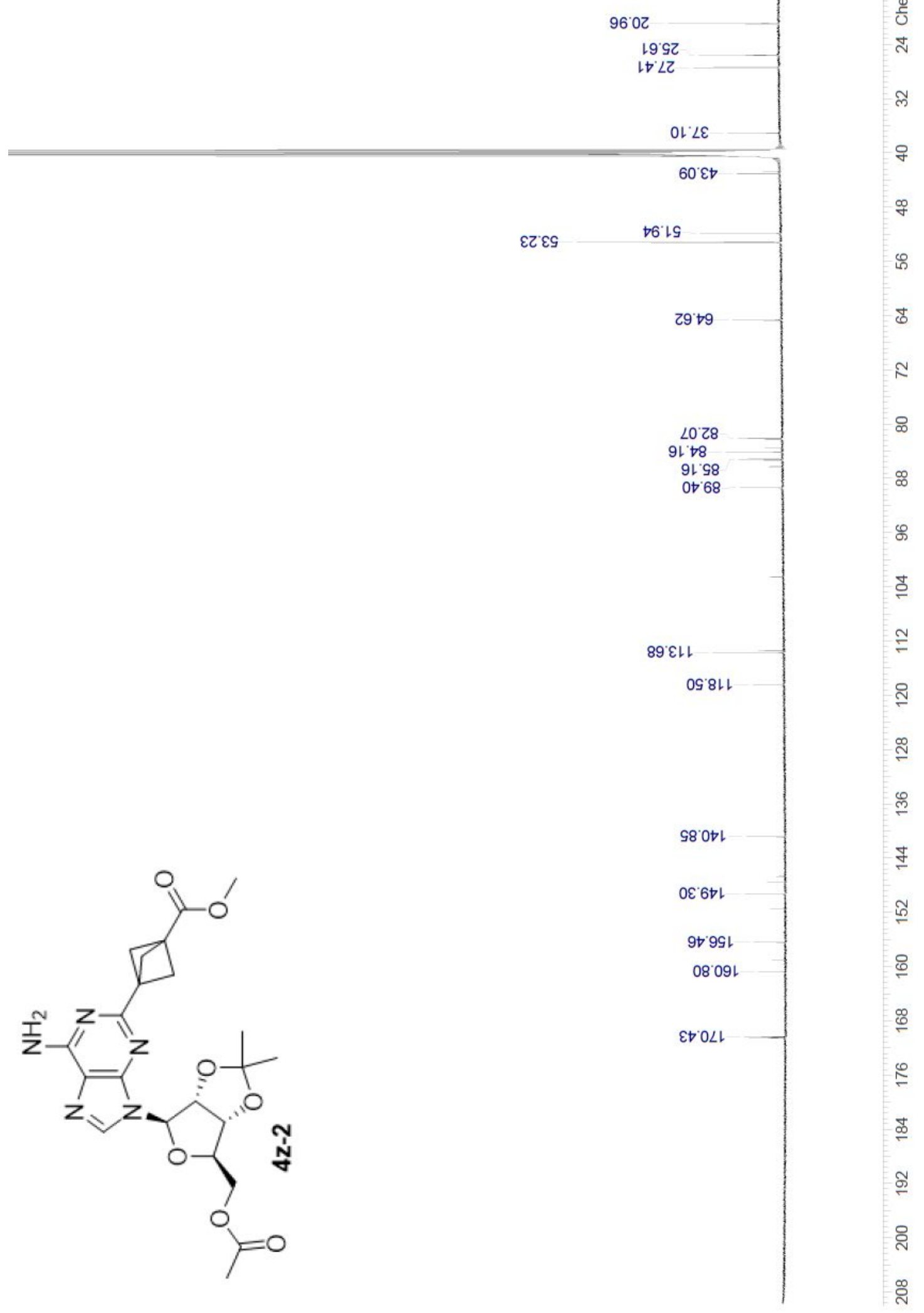




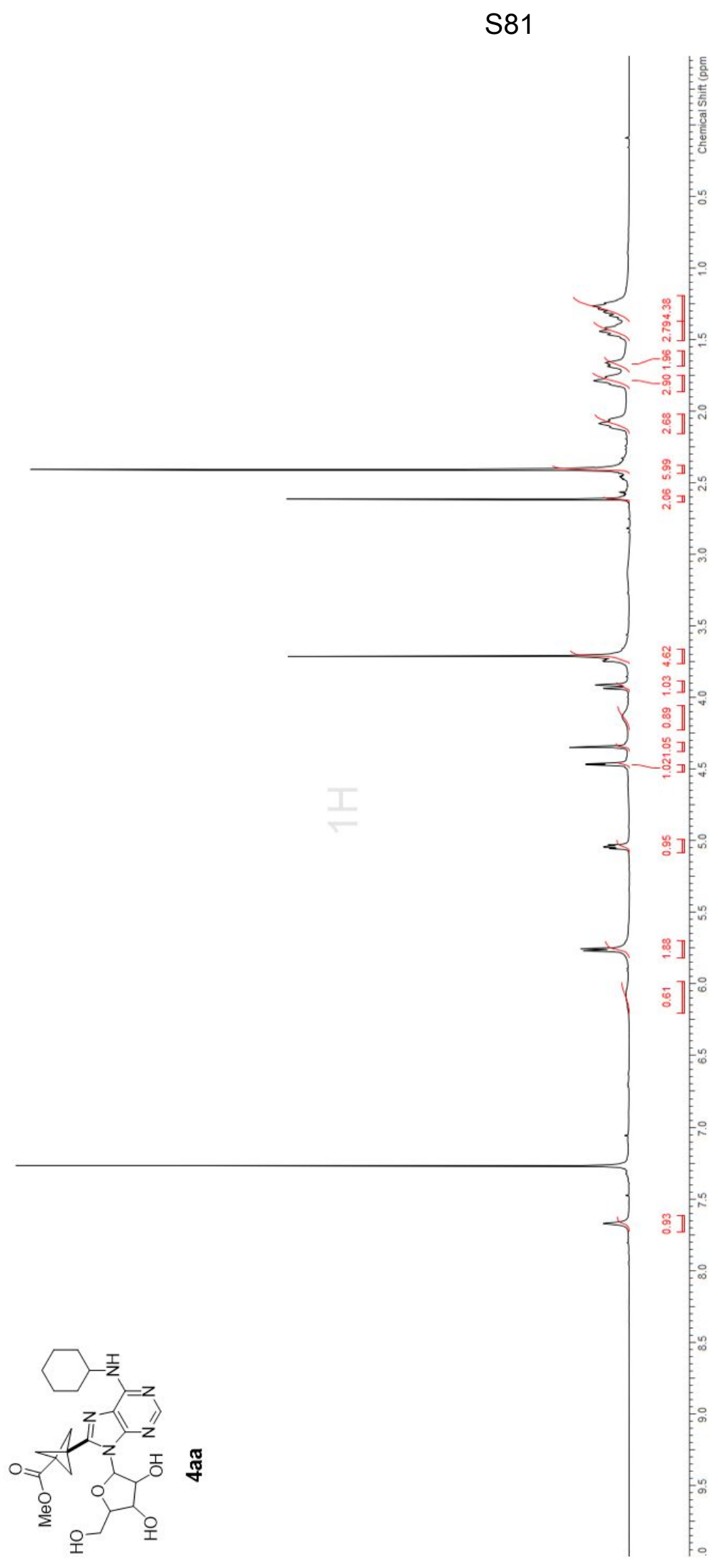




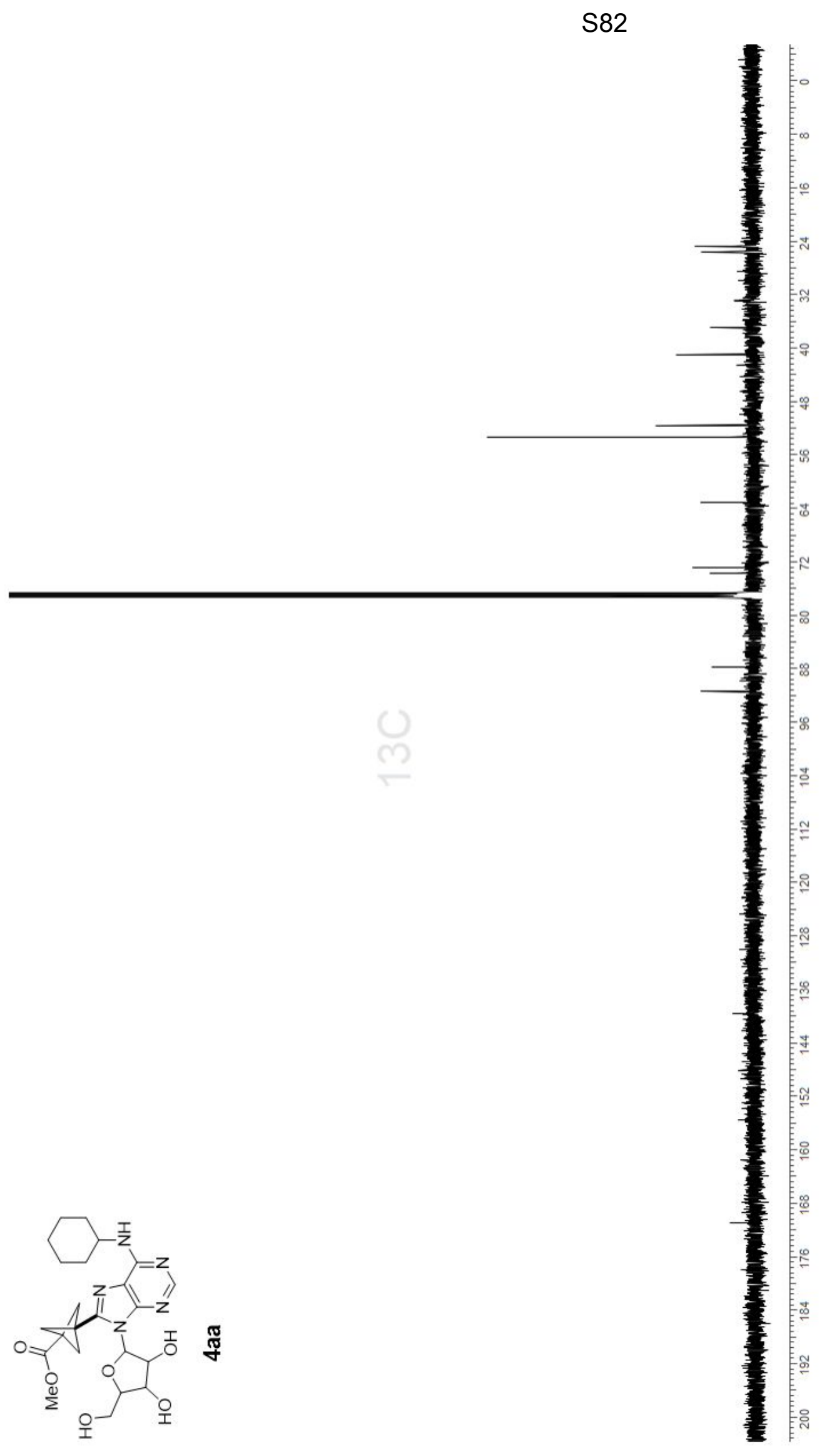


S83

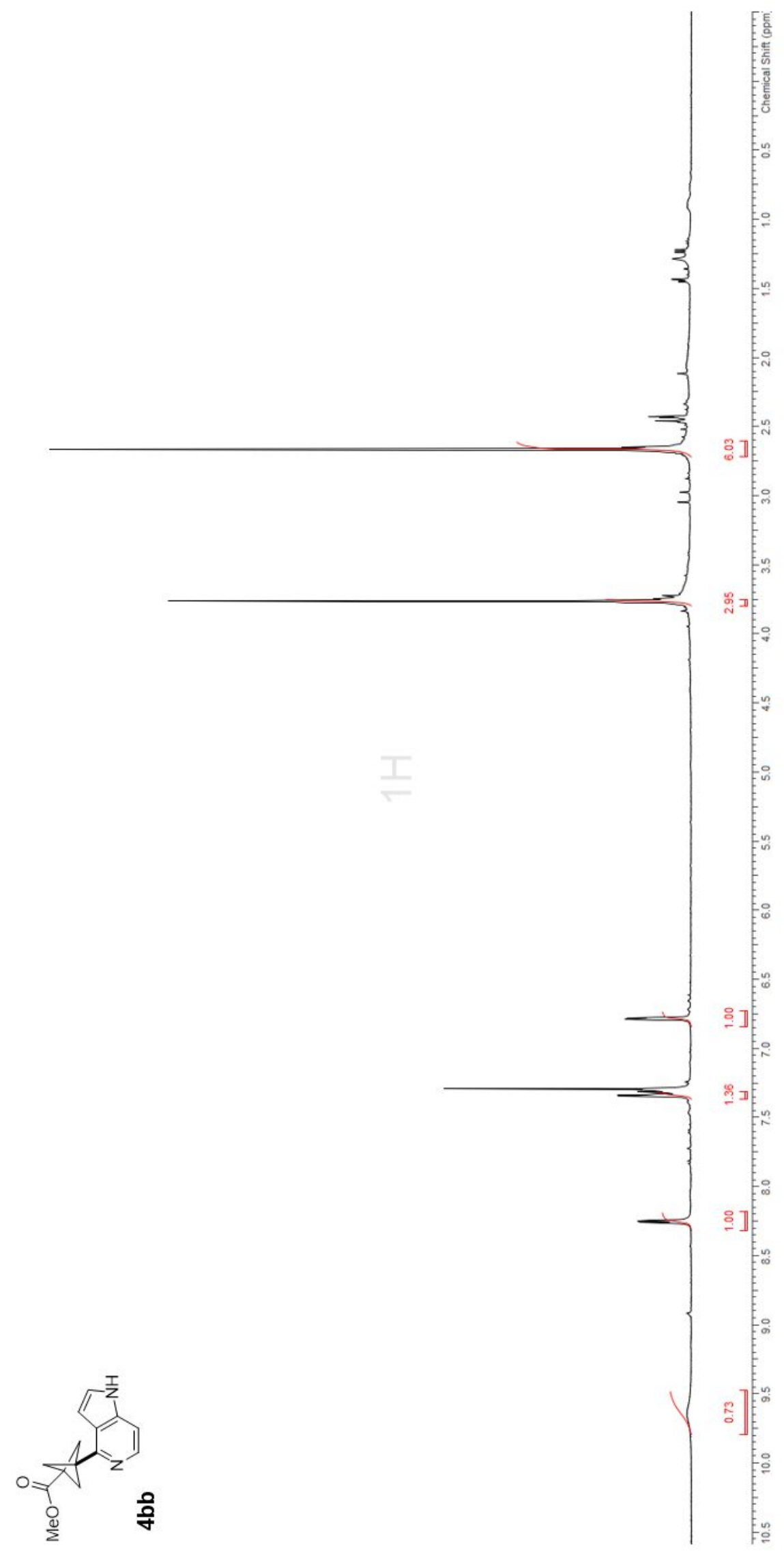




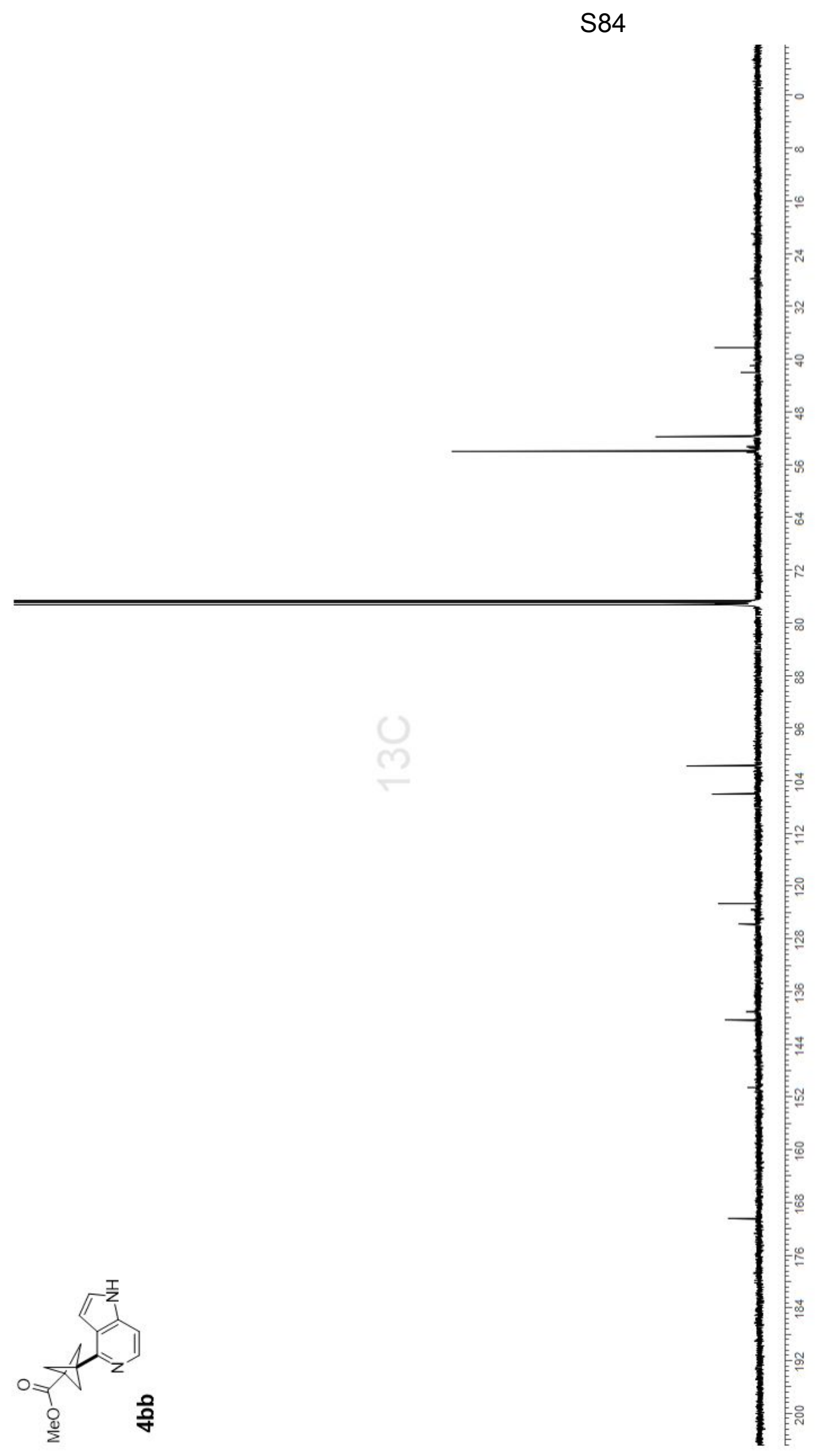




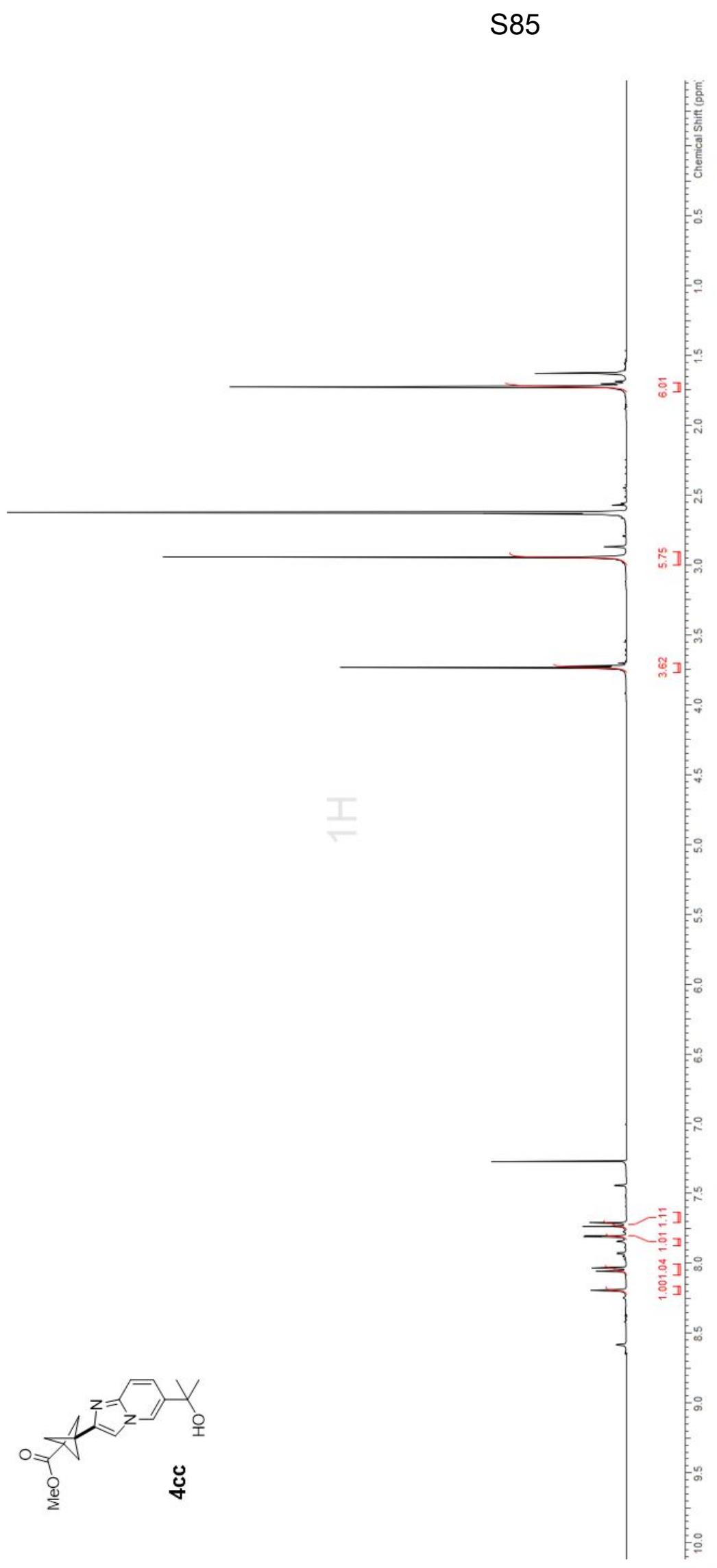




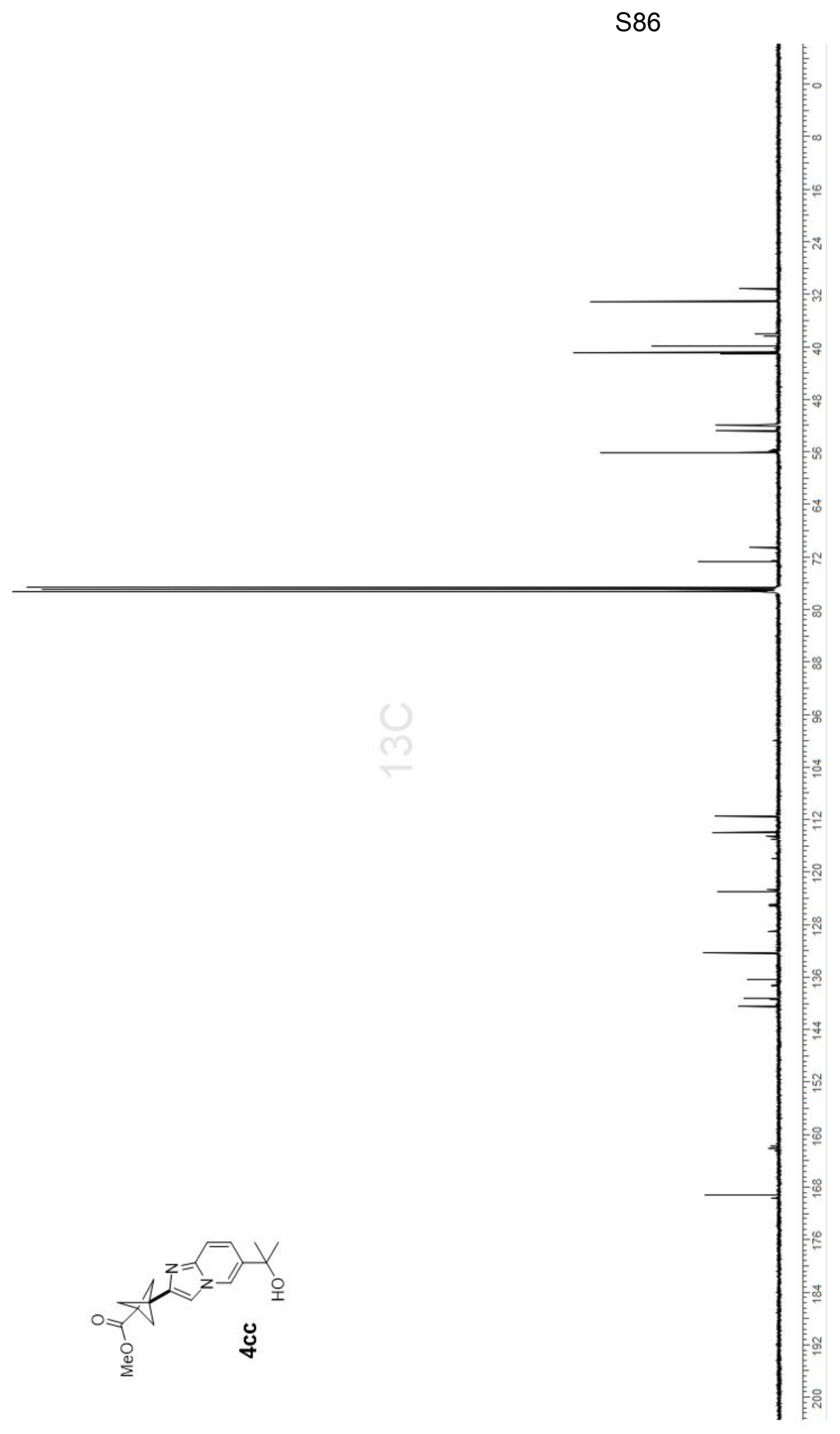




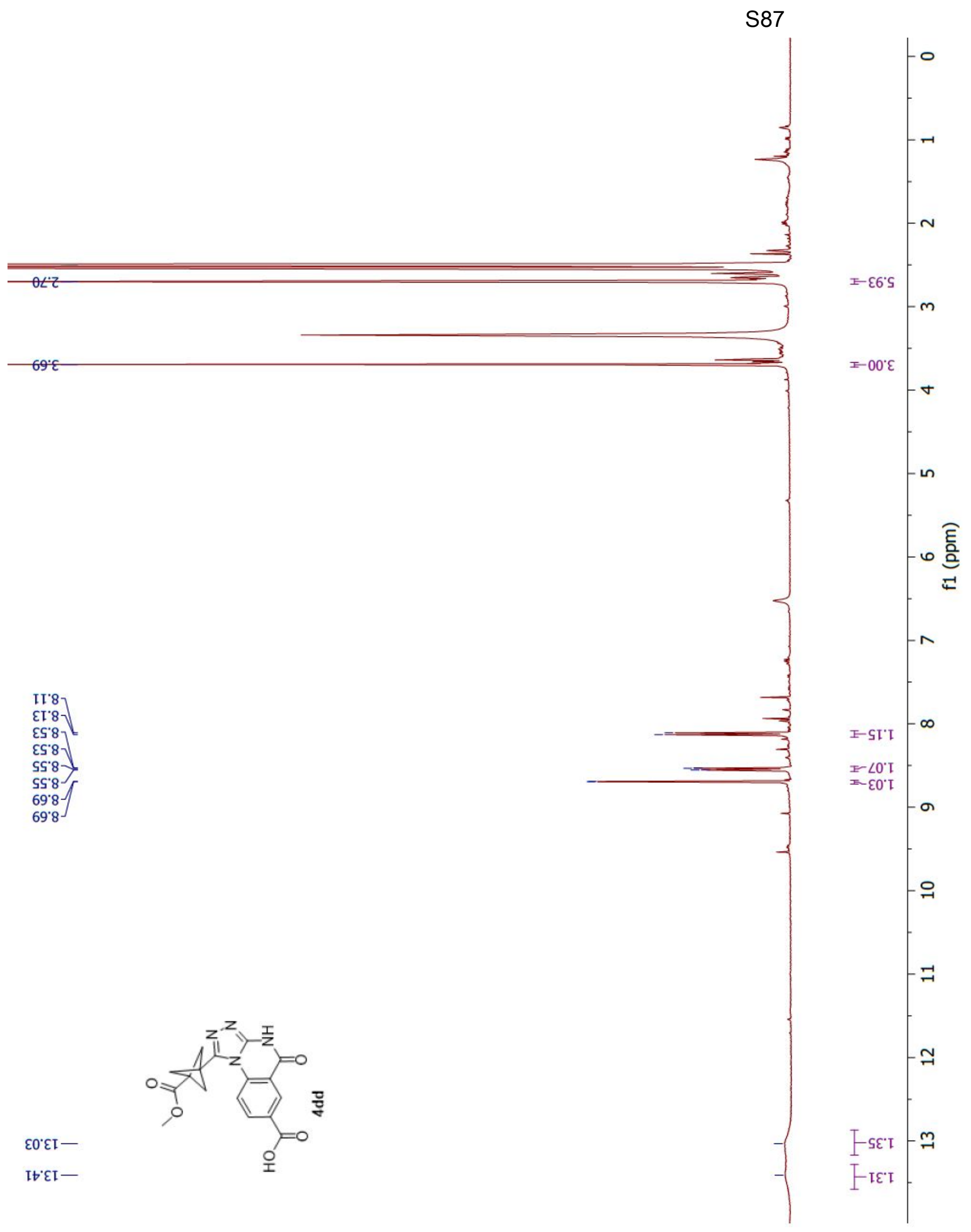




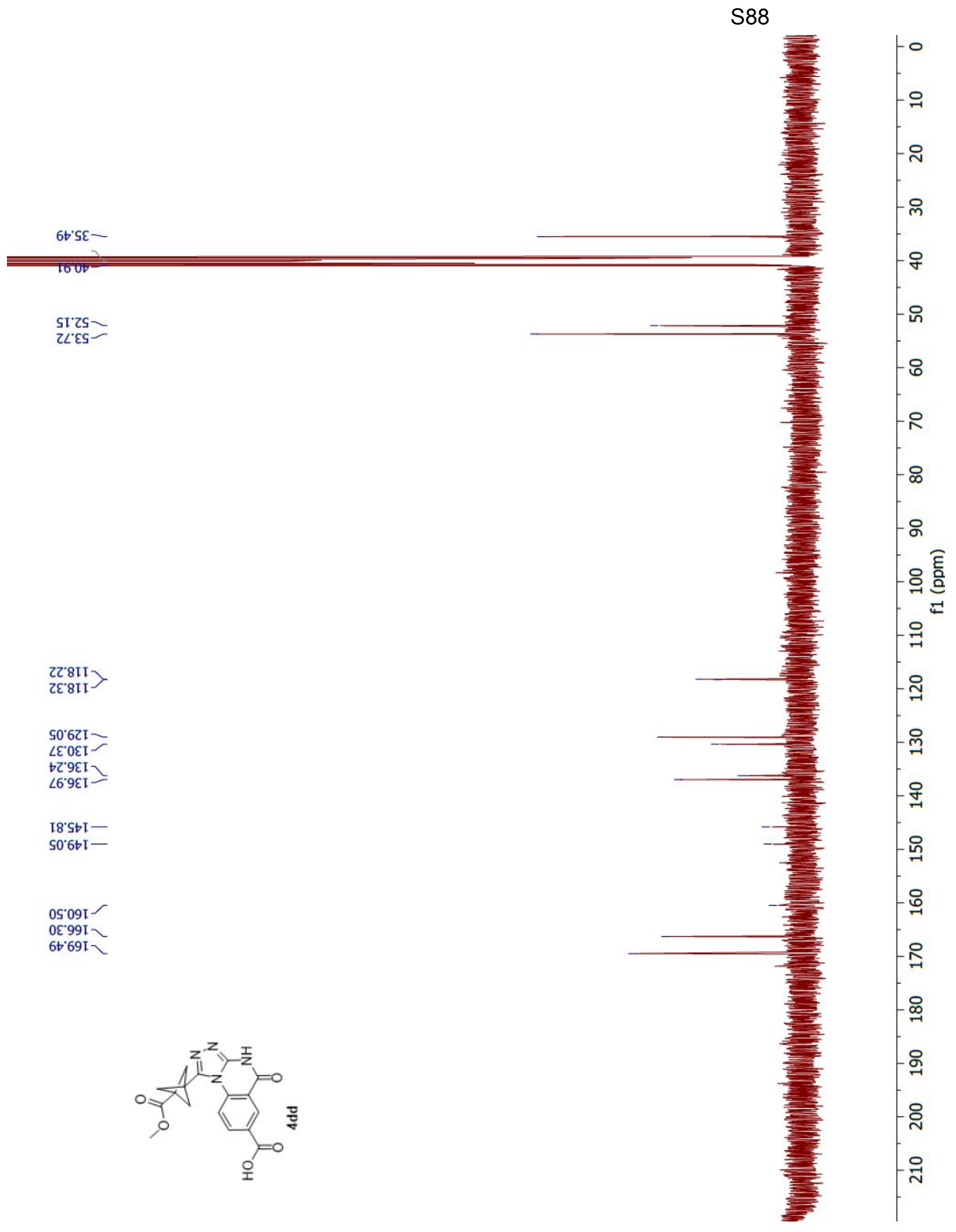




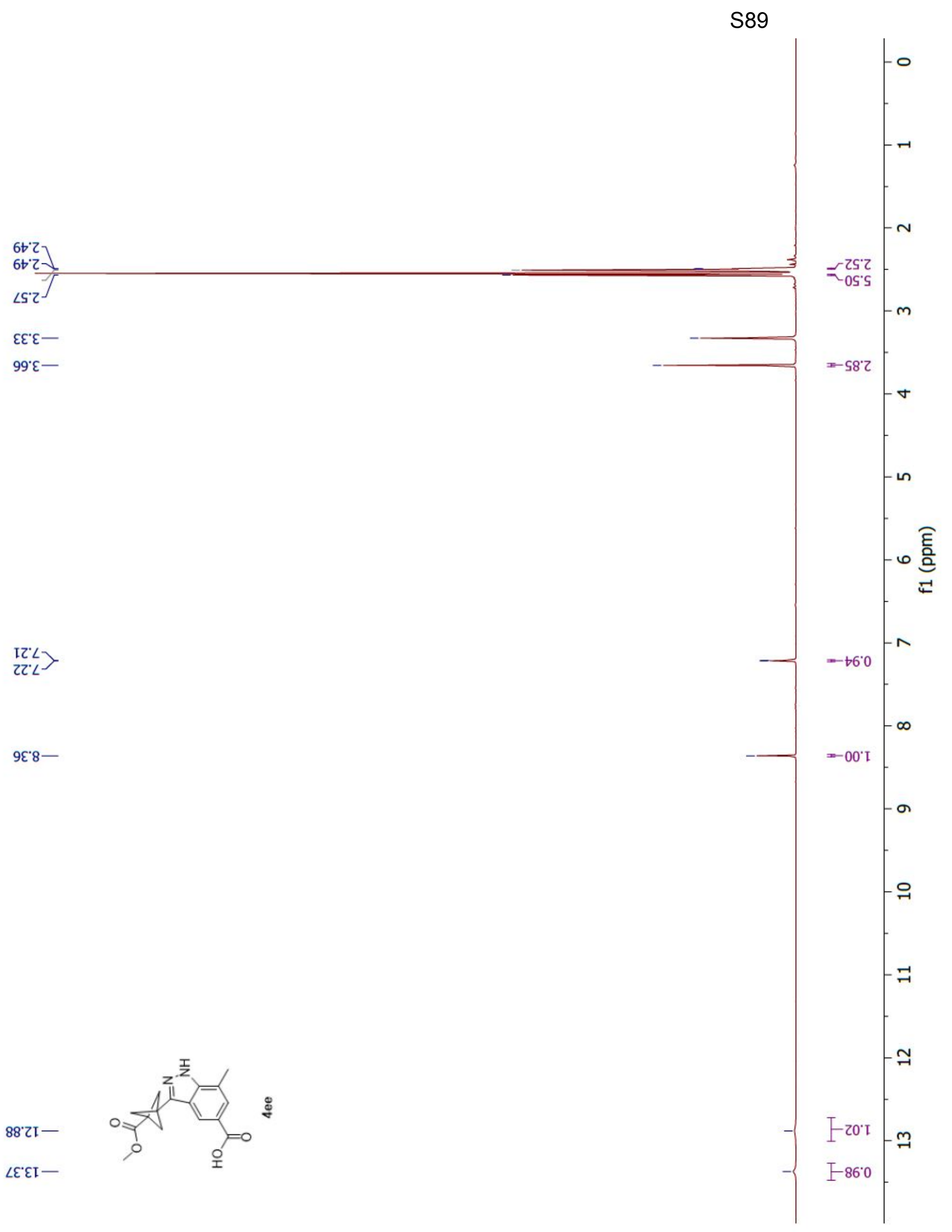




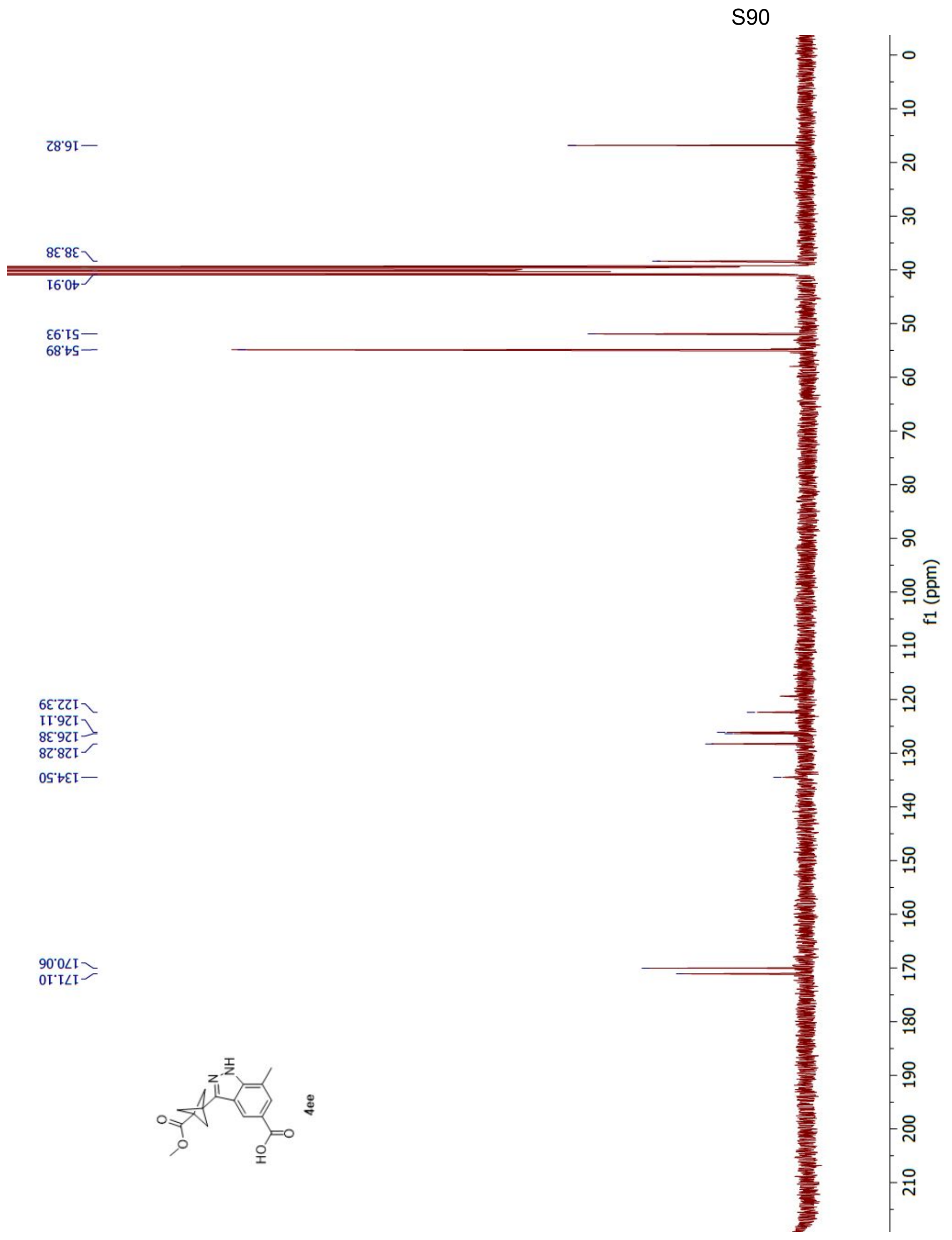




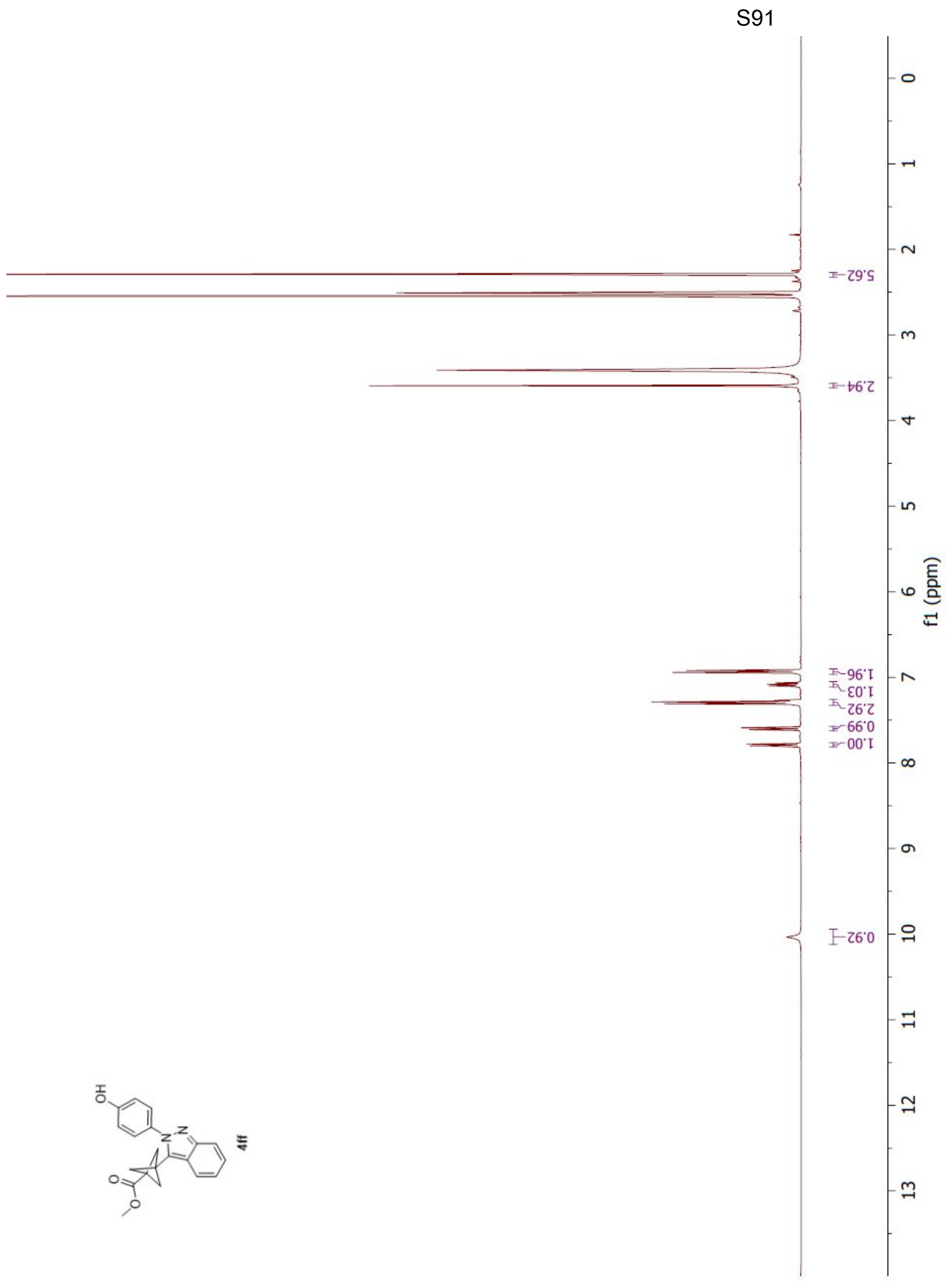




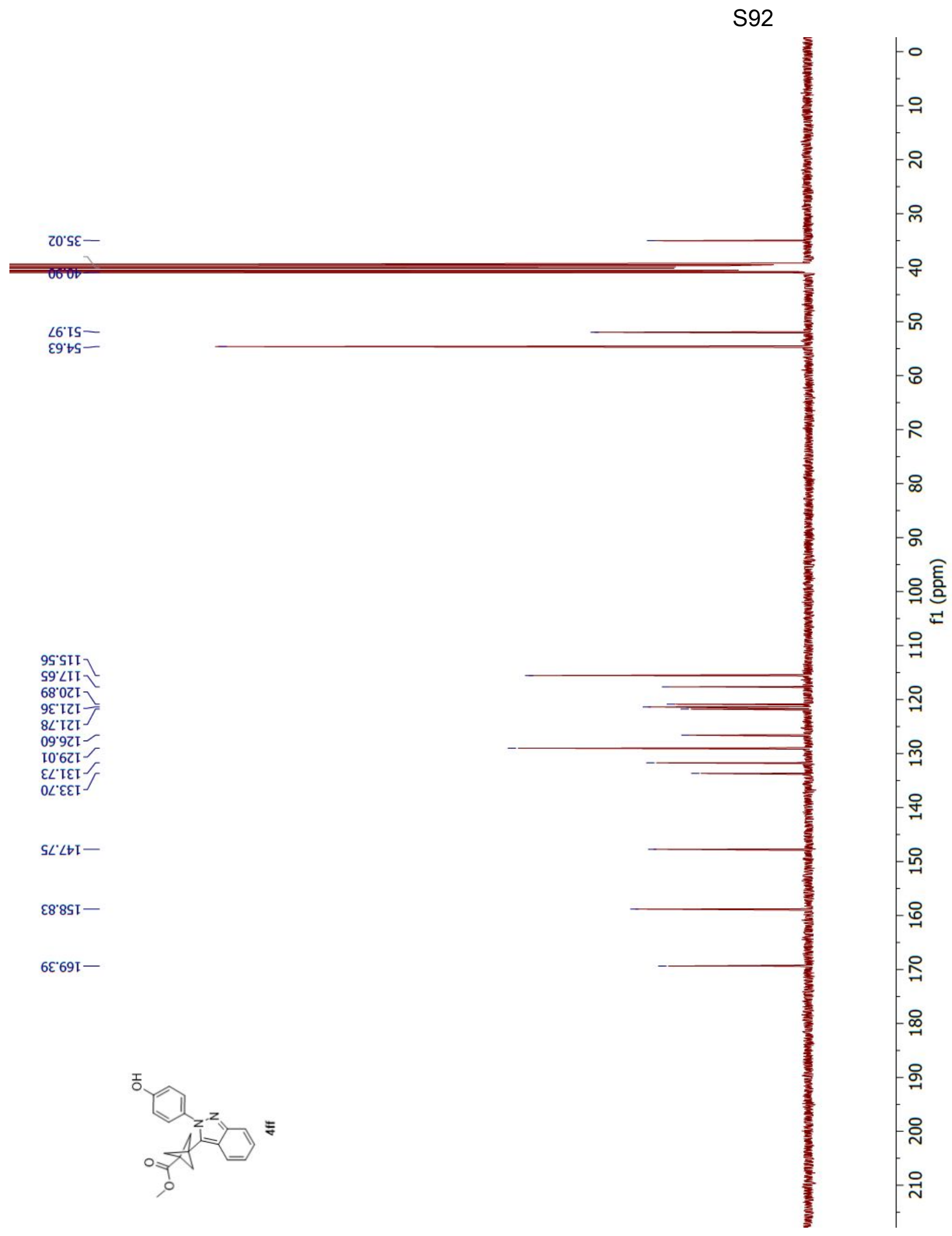


S93

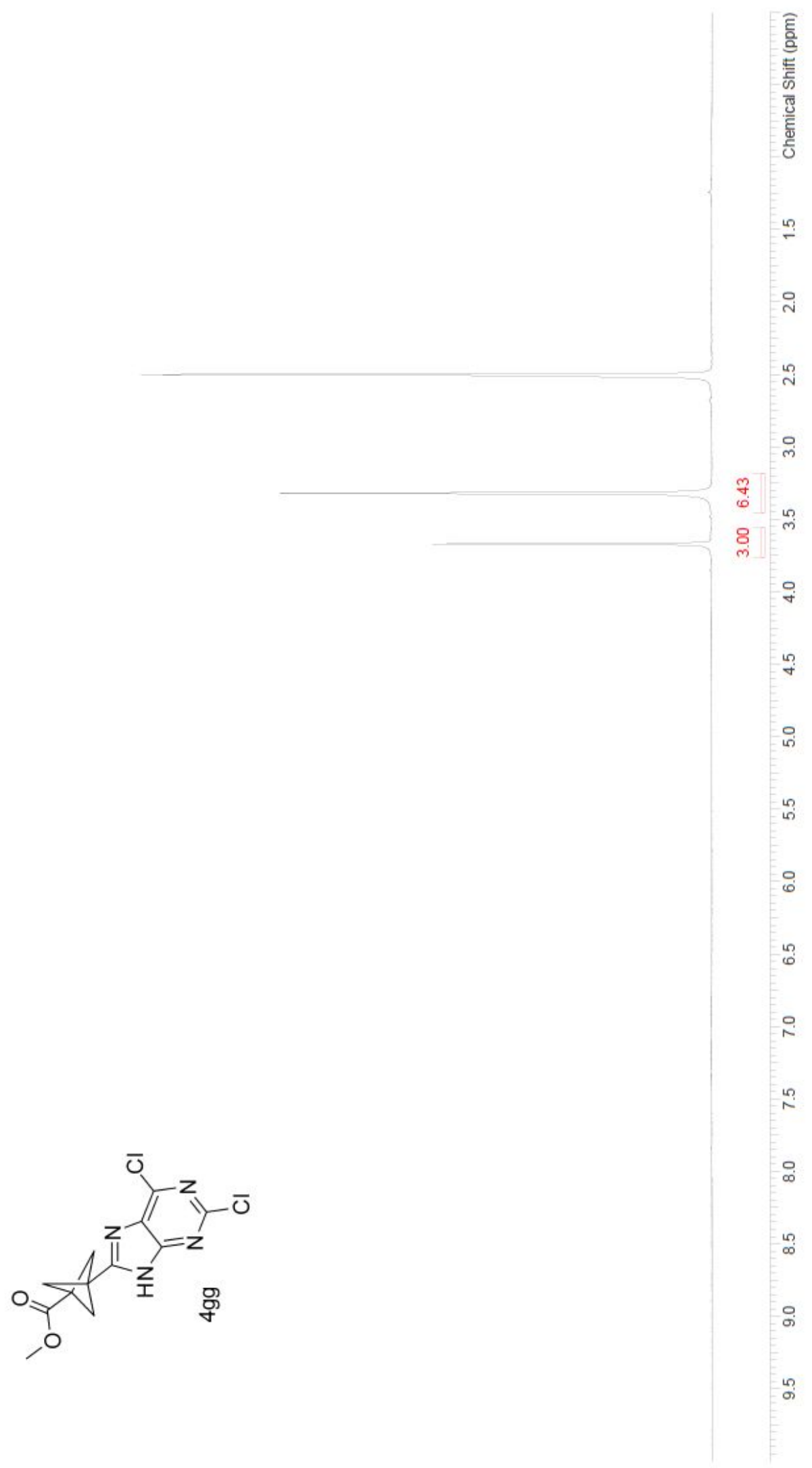


S94

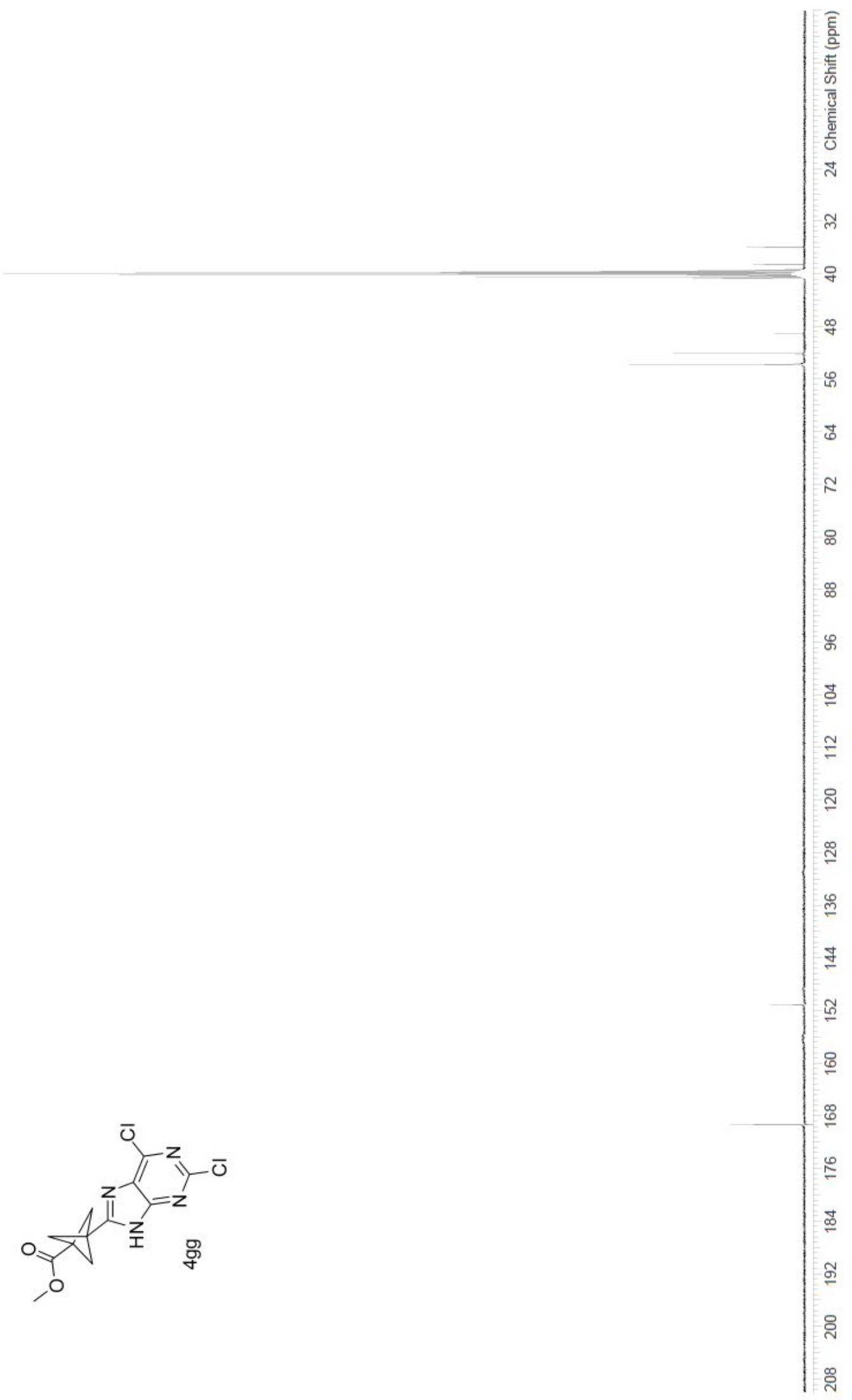




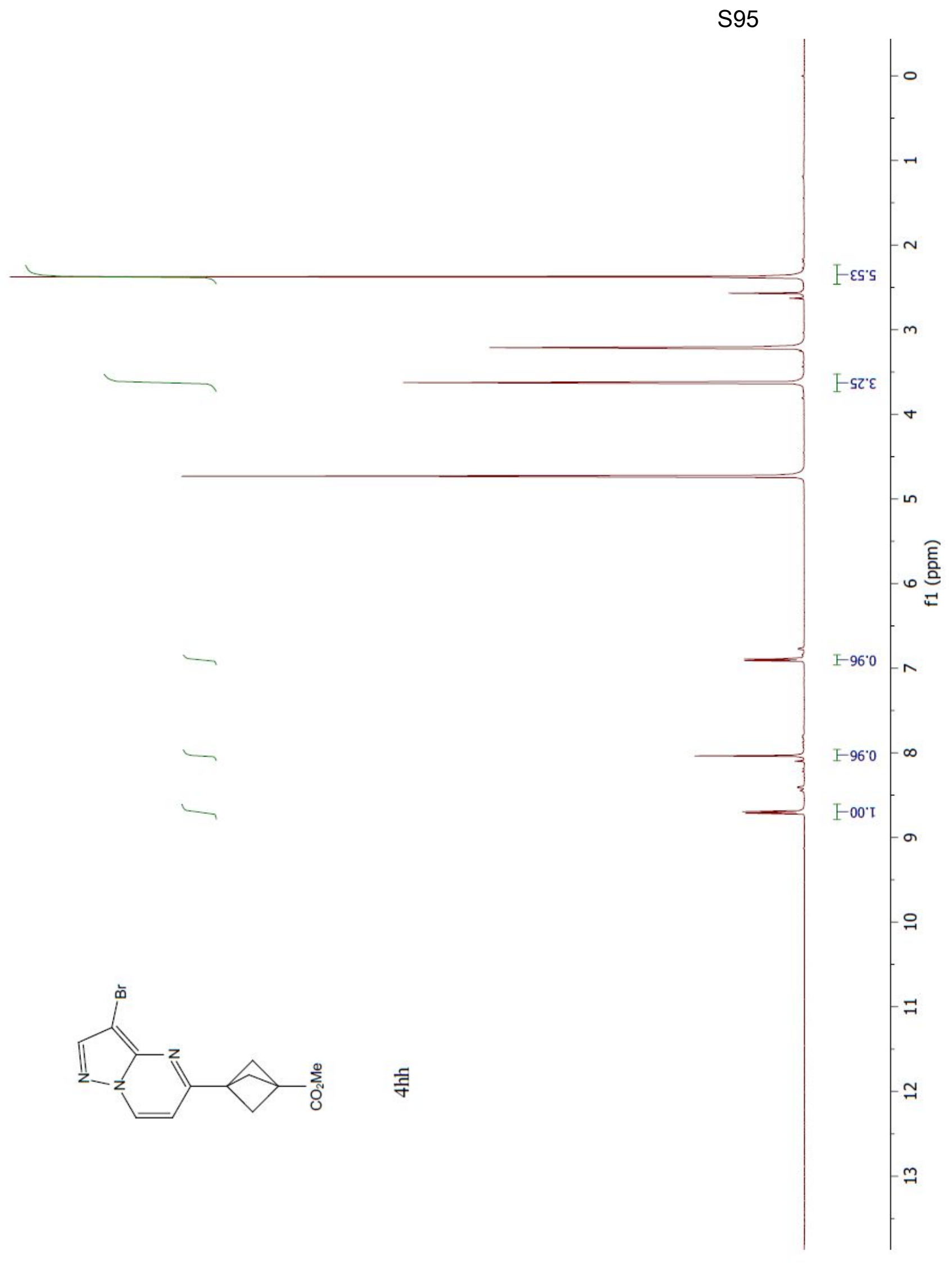




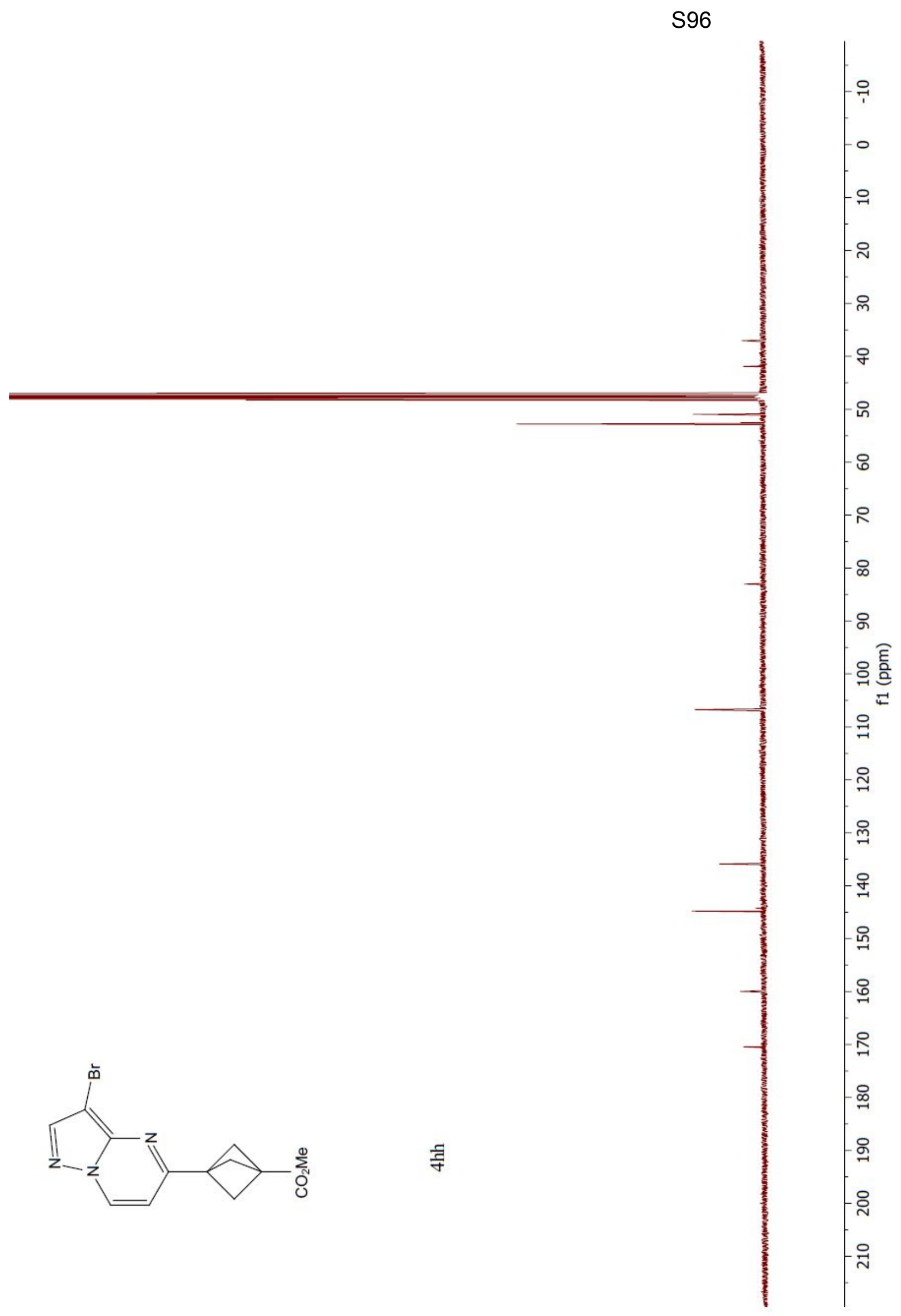




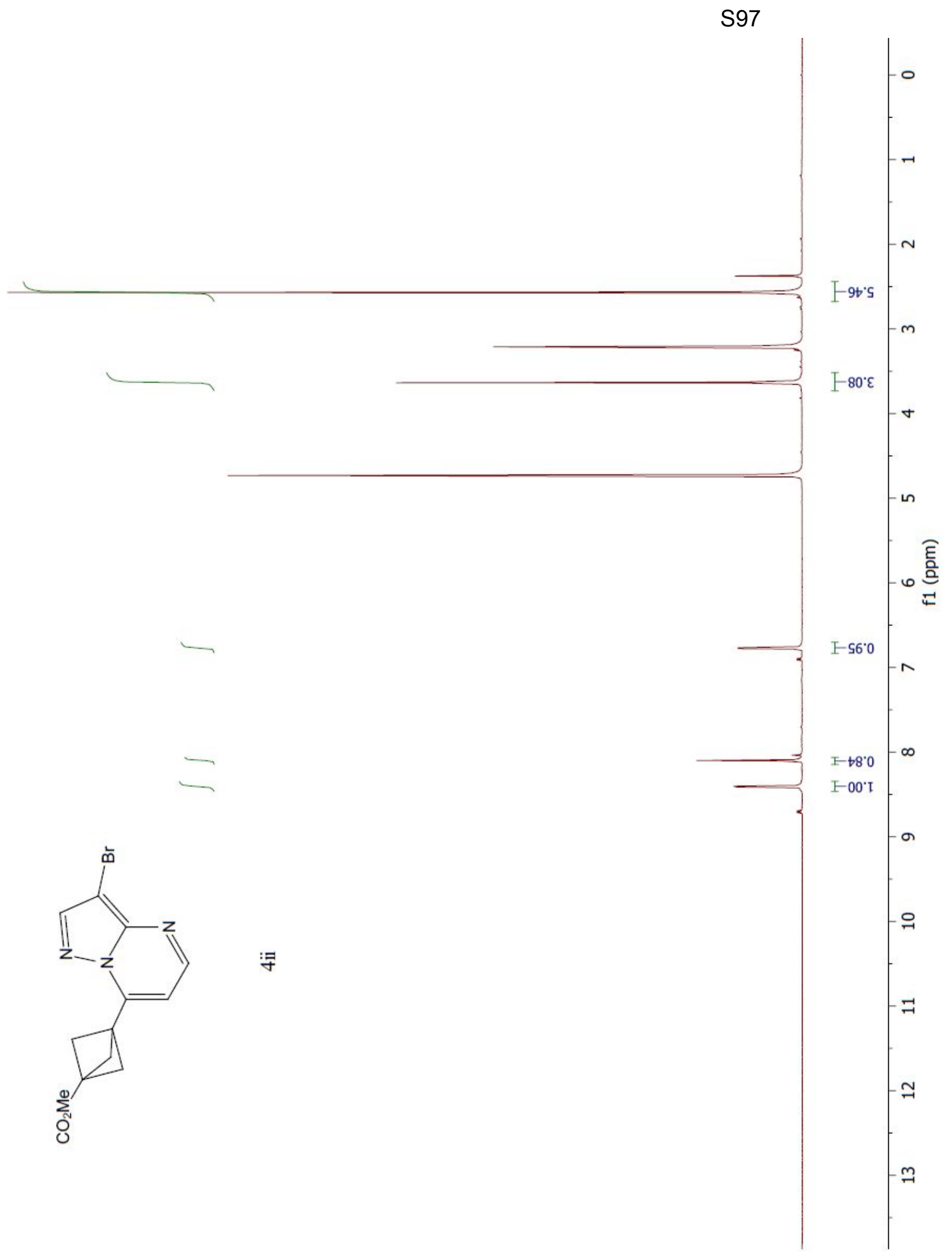




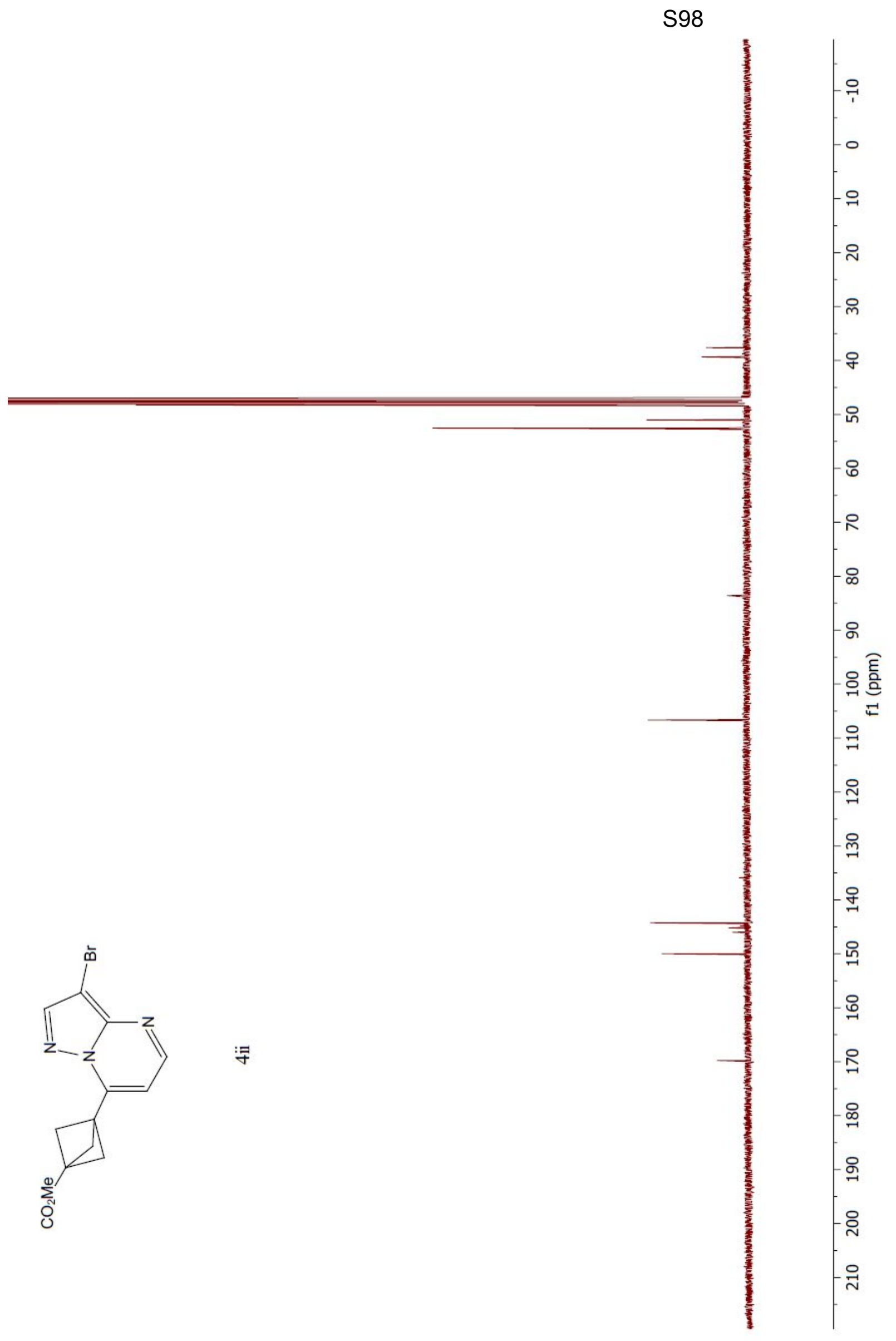




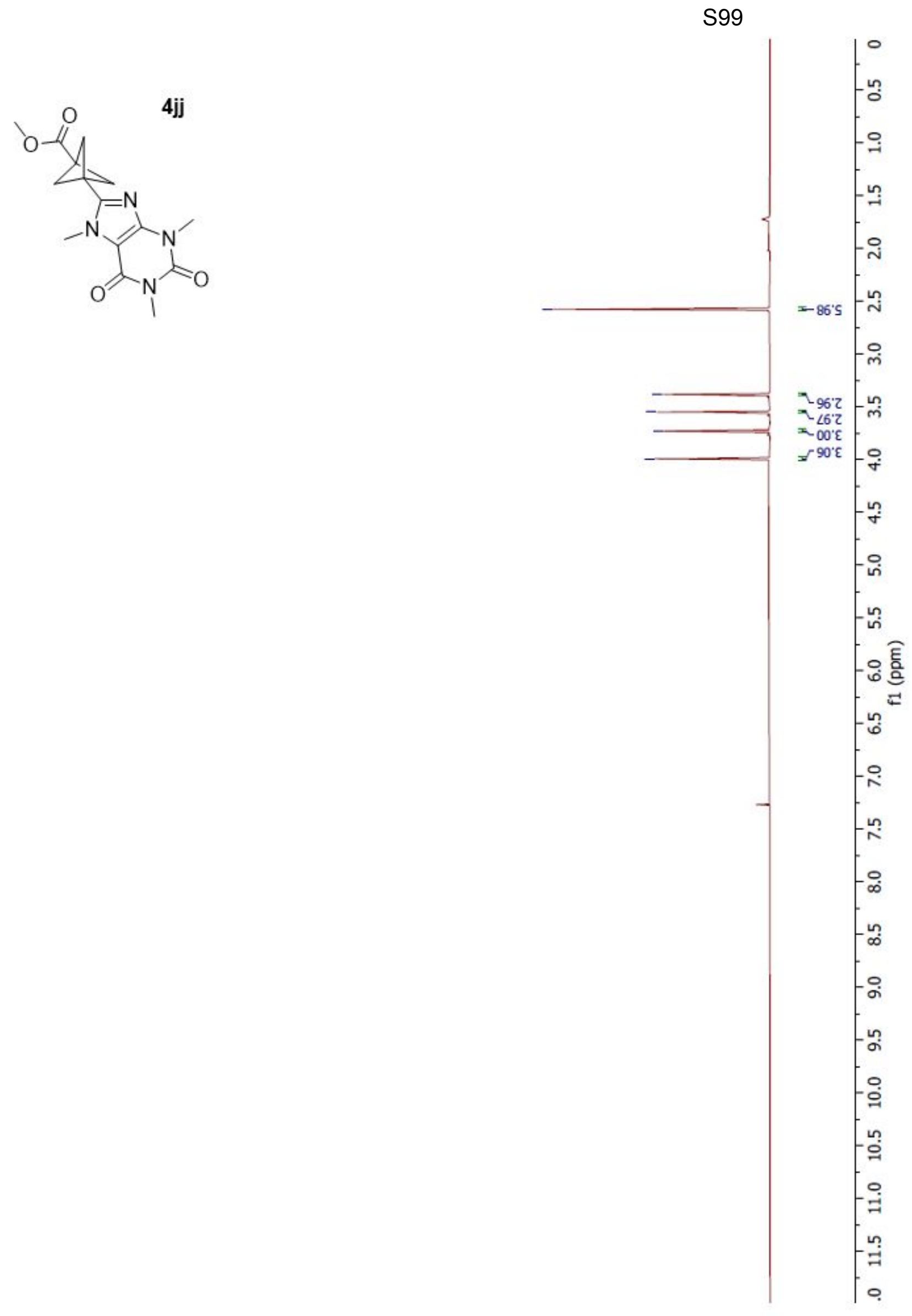




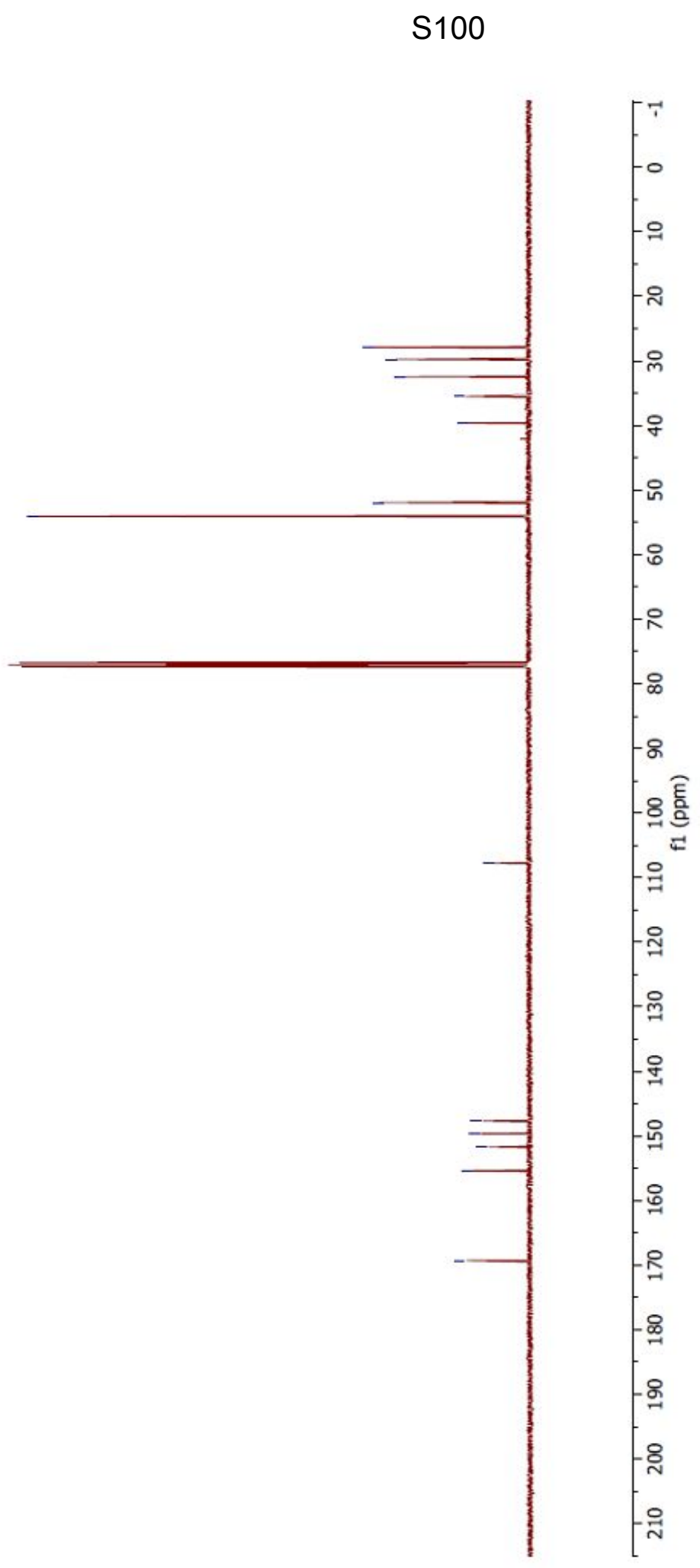



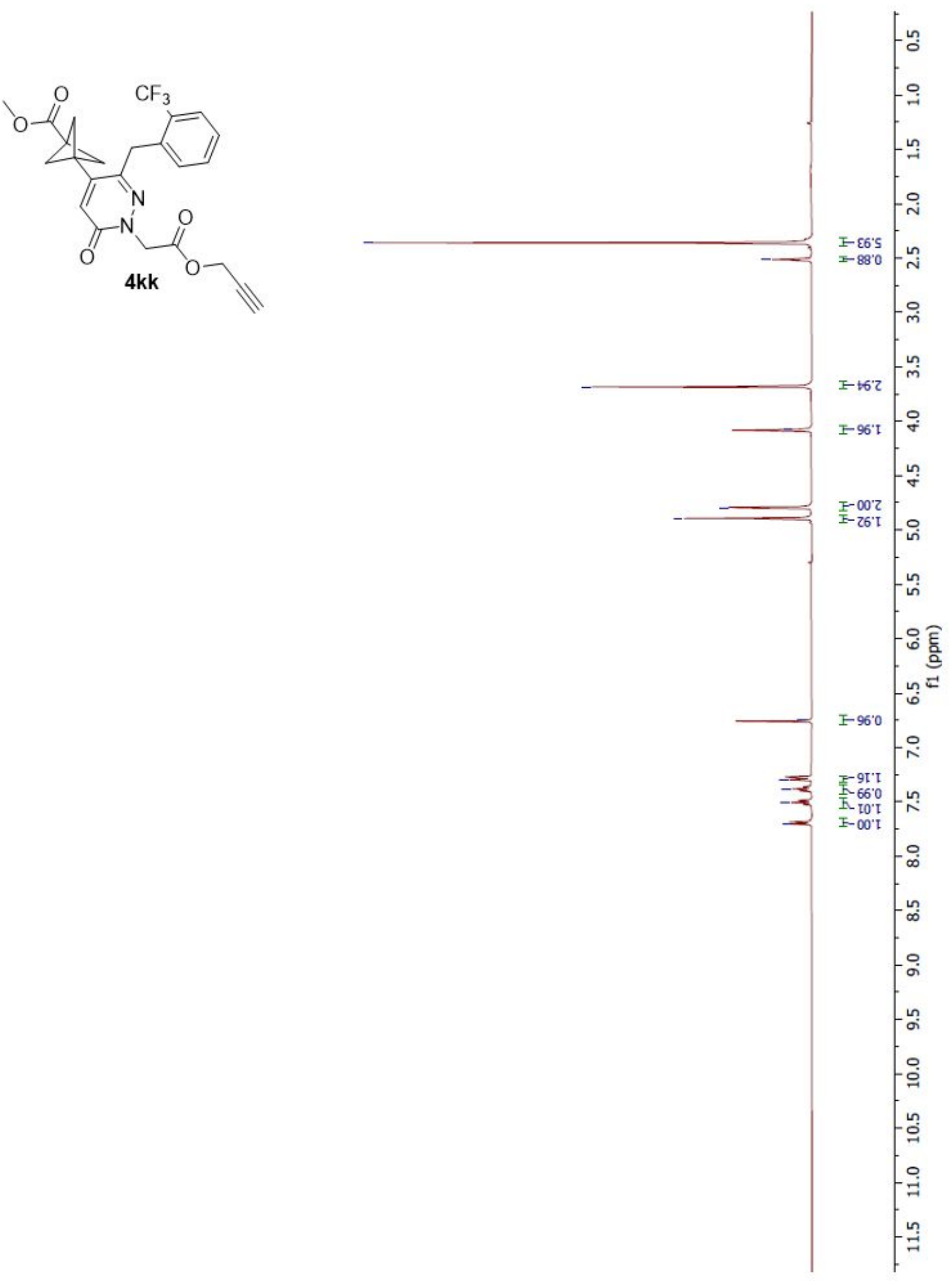


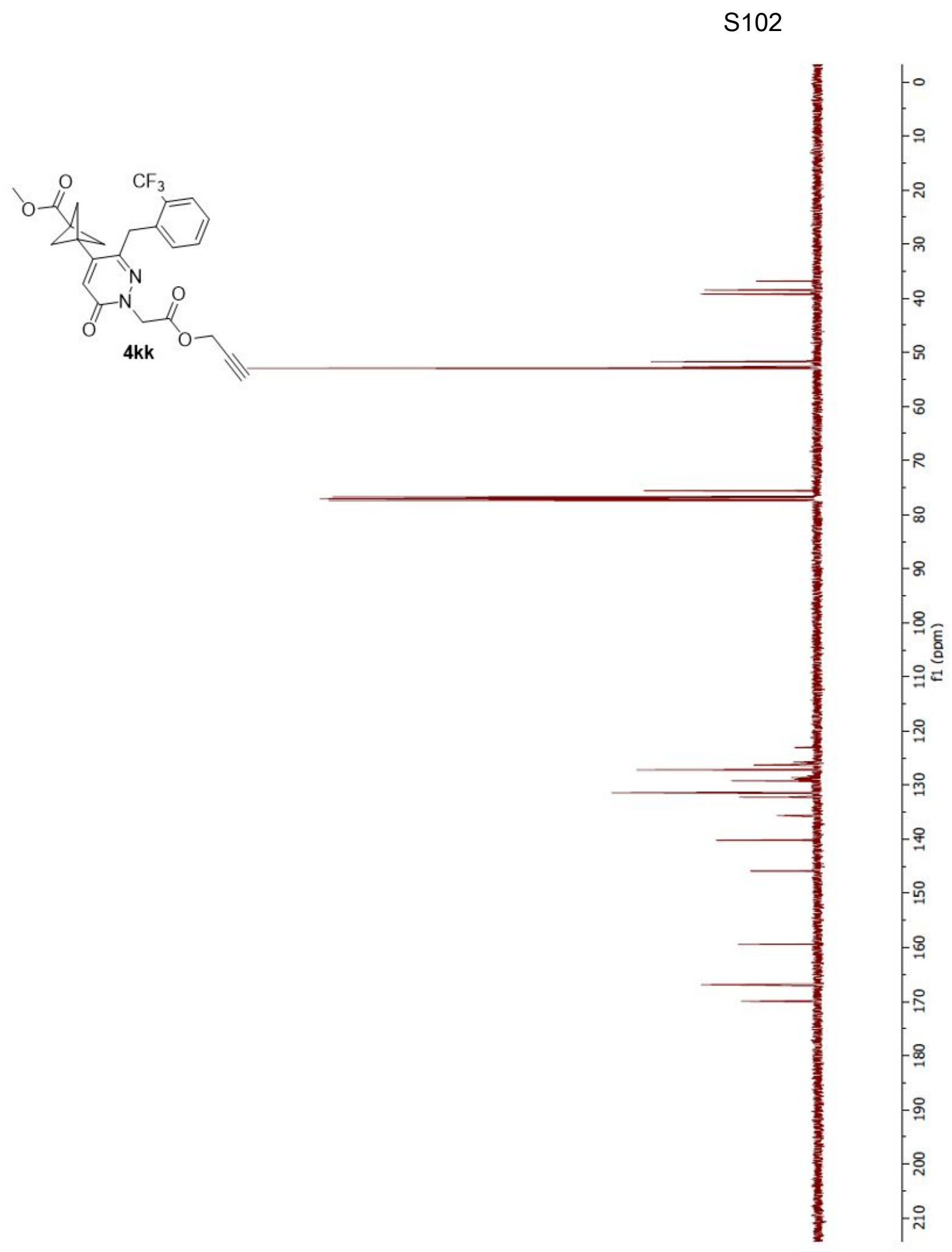


S103
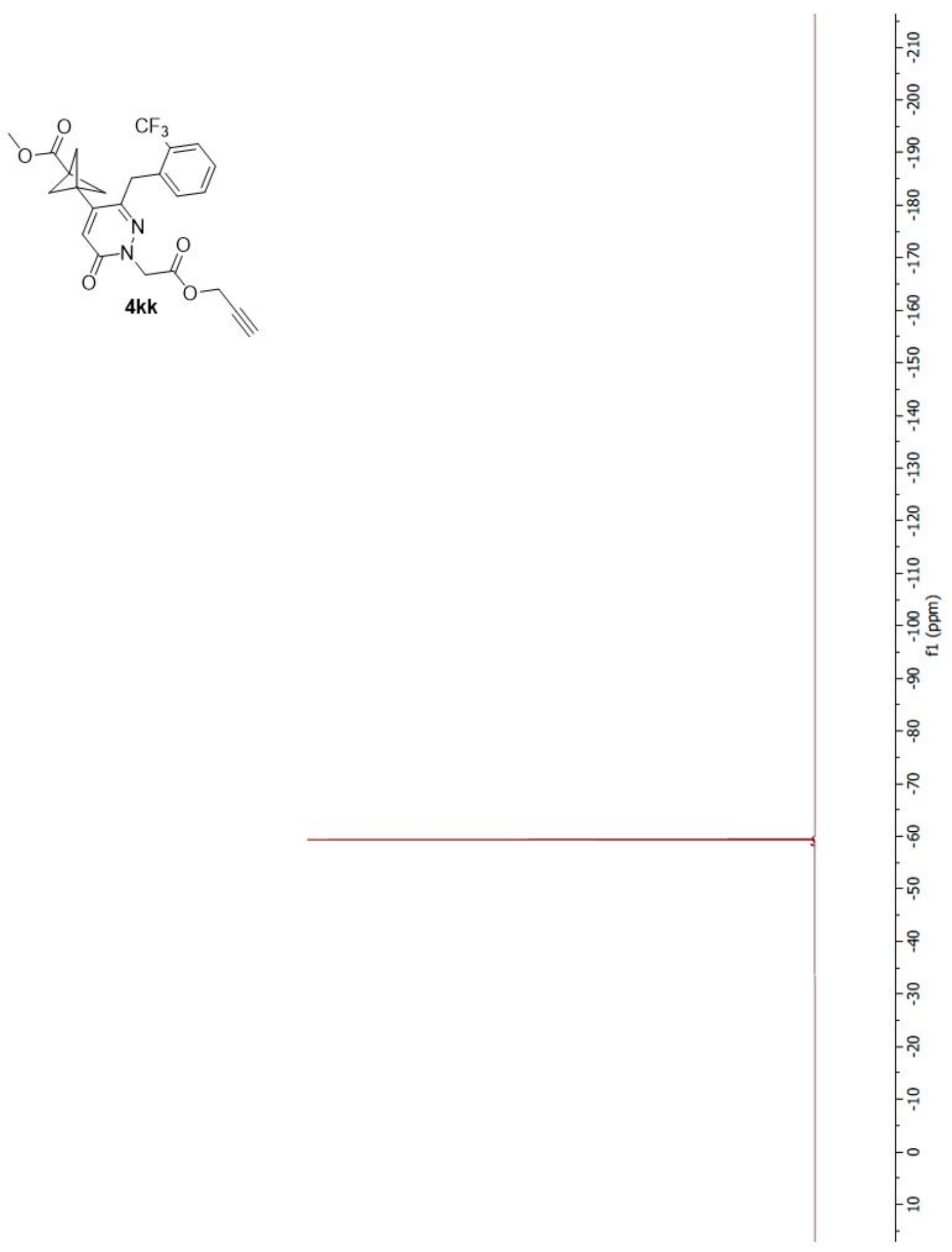

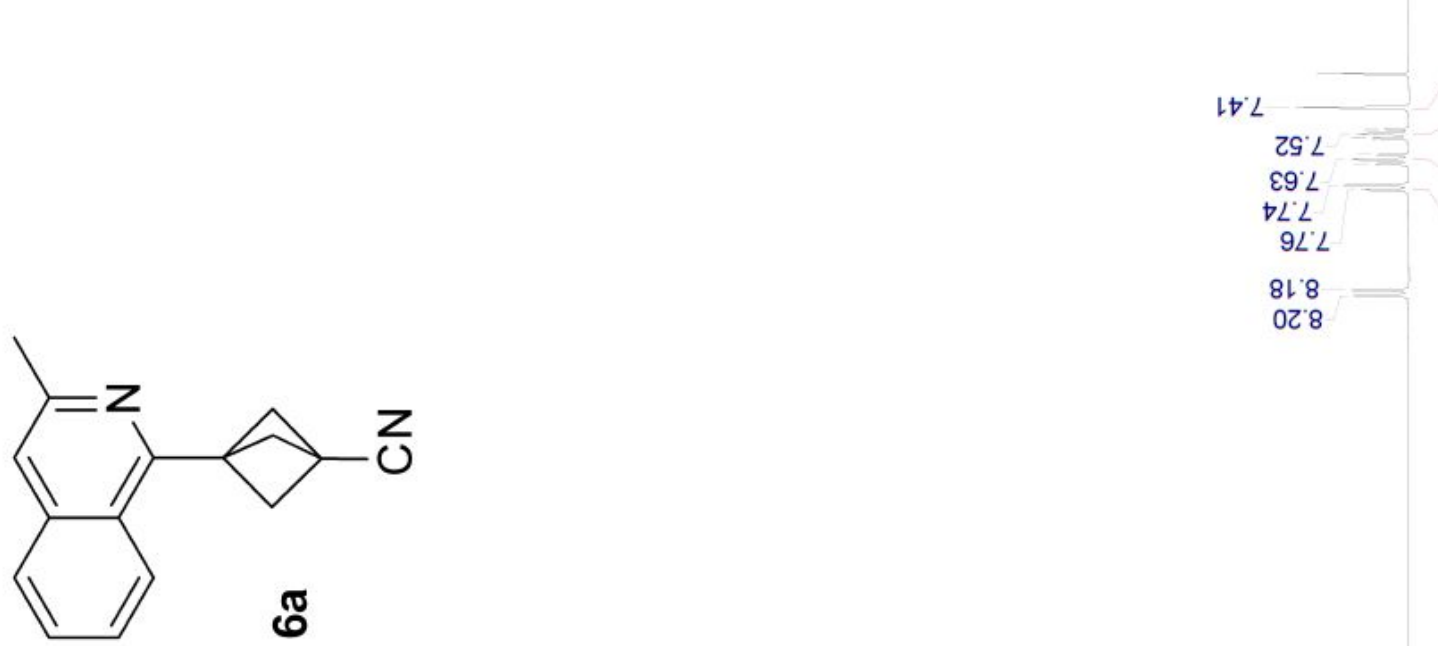


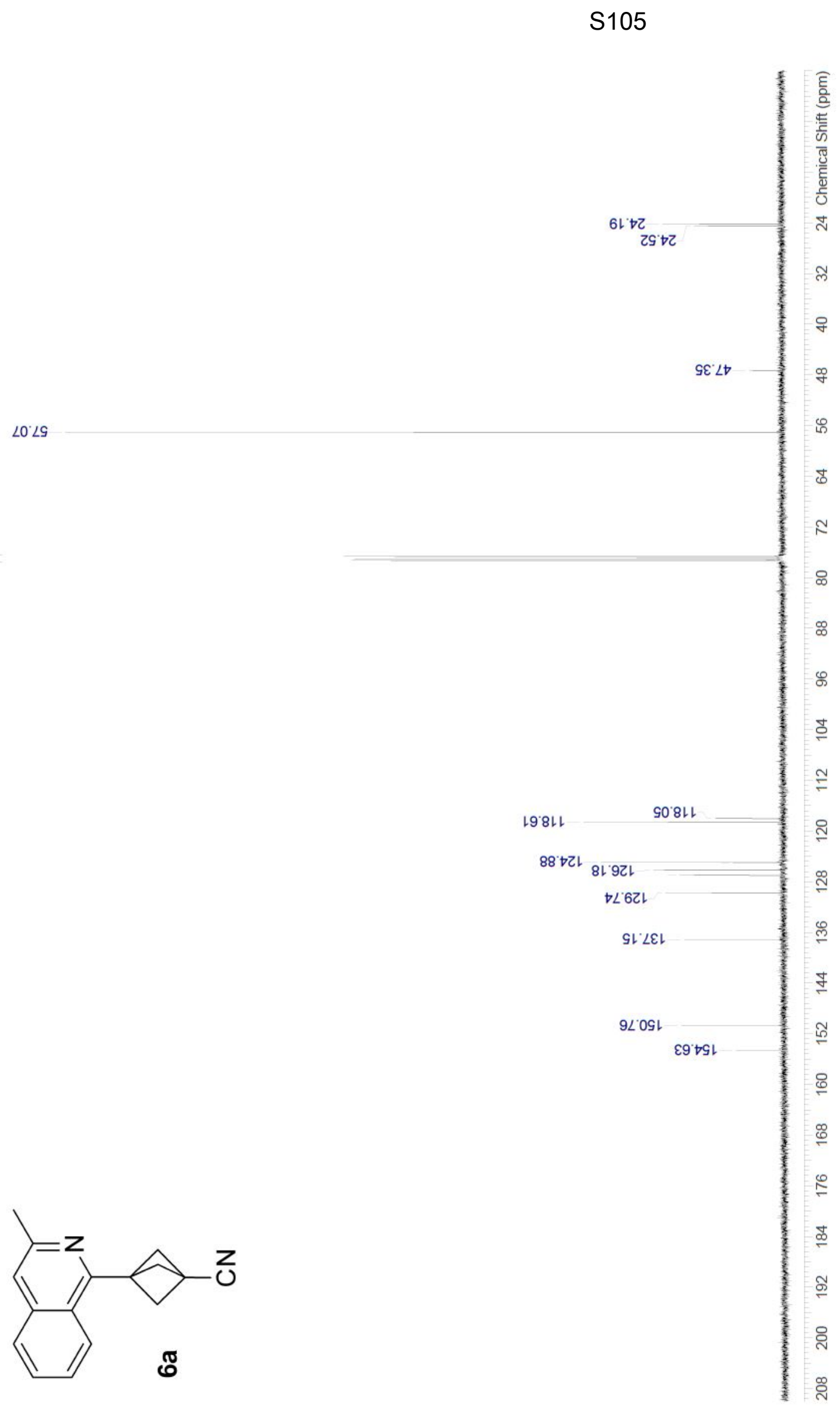


$0 \varepsilon^{\prime} 乙$

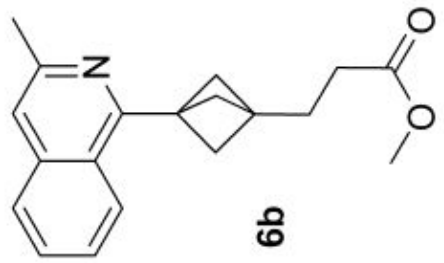

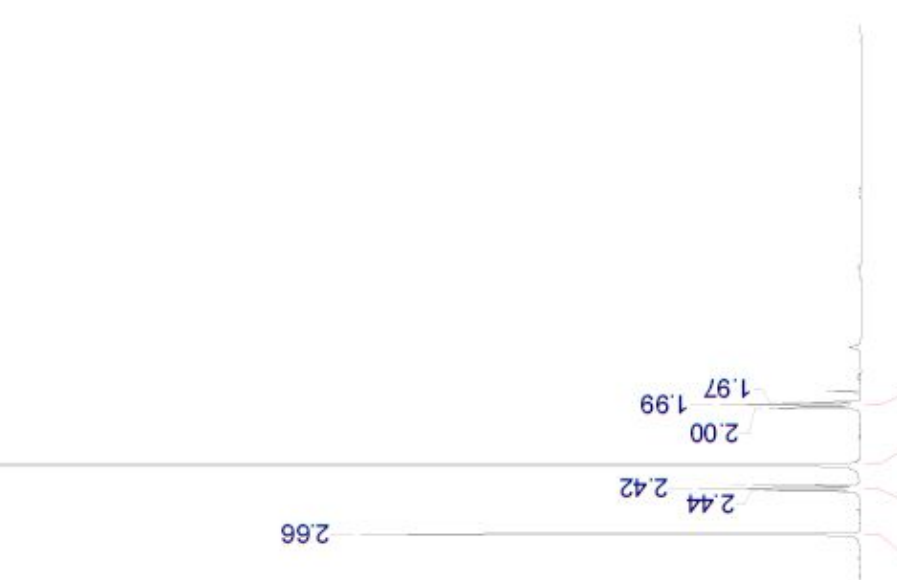

LE

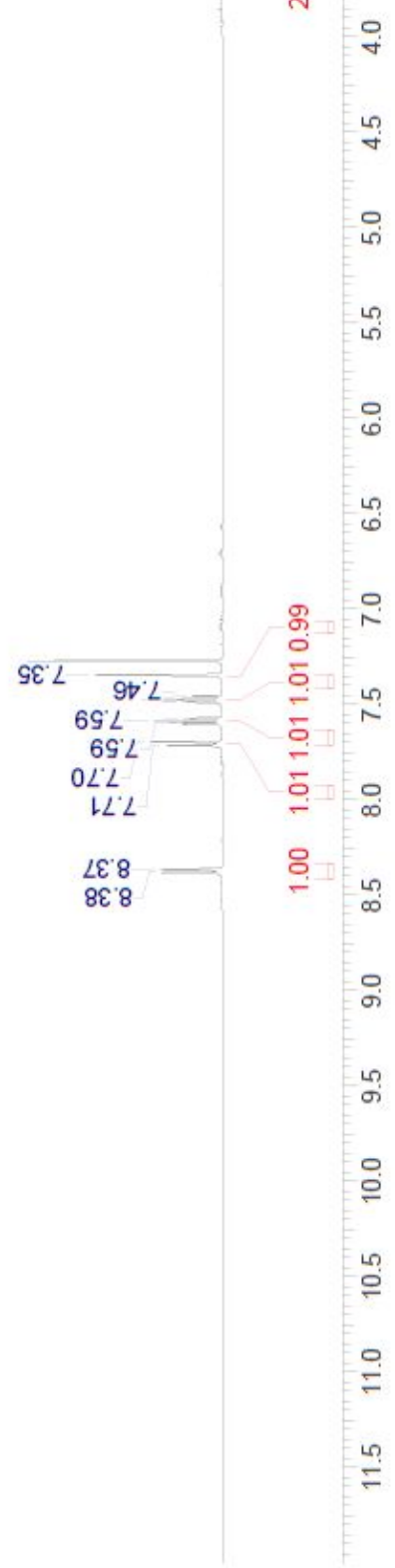

$8 \varepsilon^{\prime} 8$ 


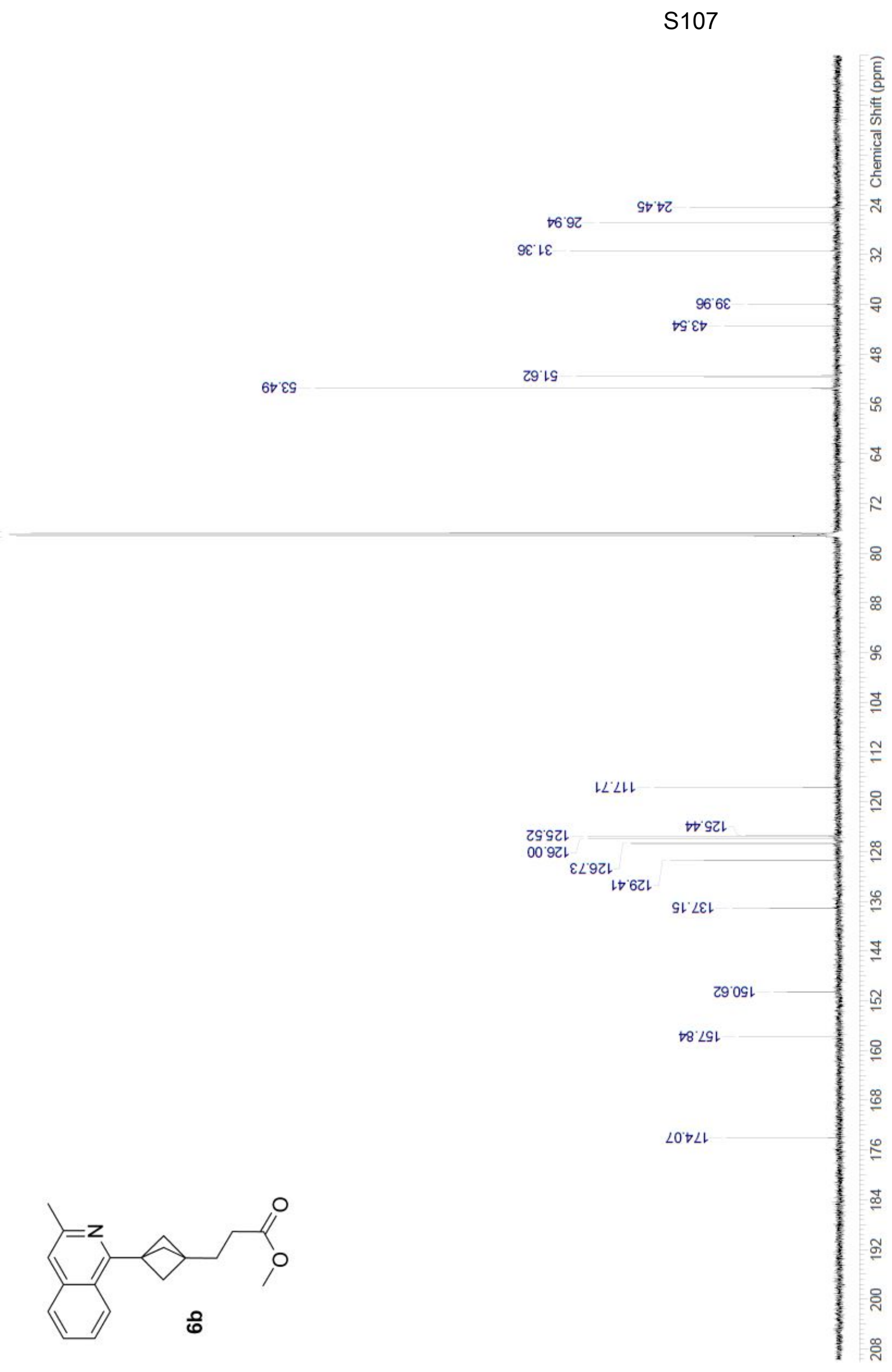




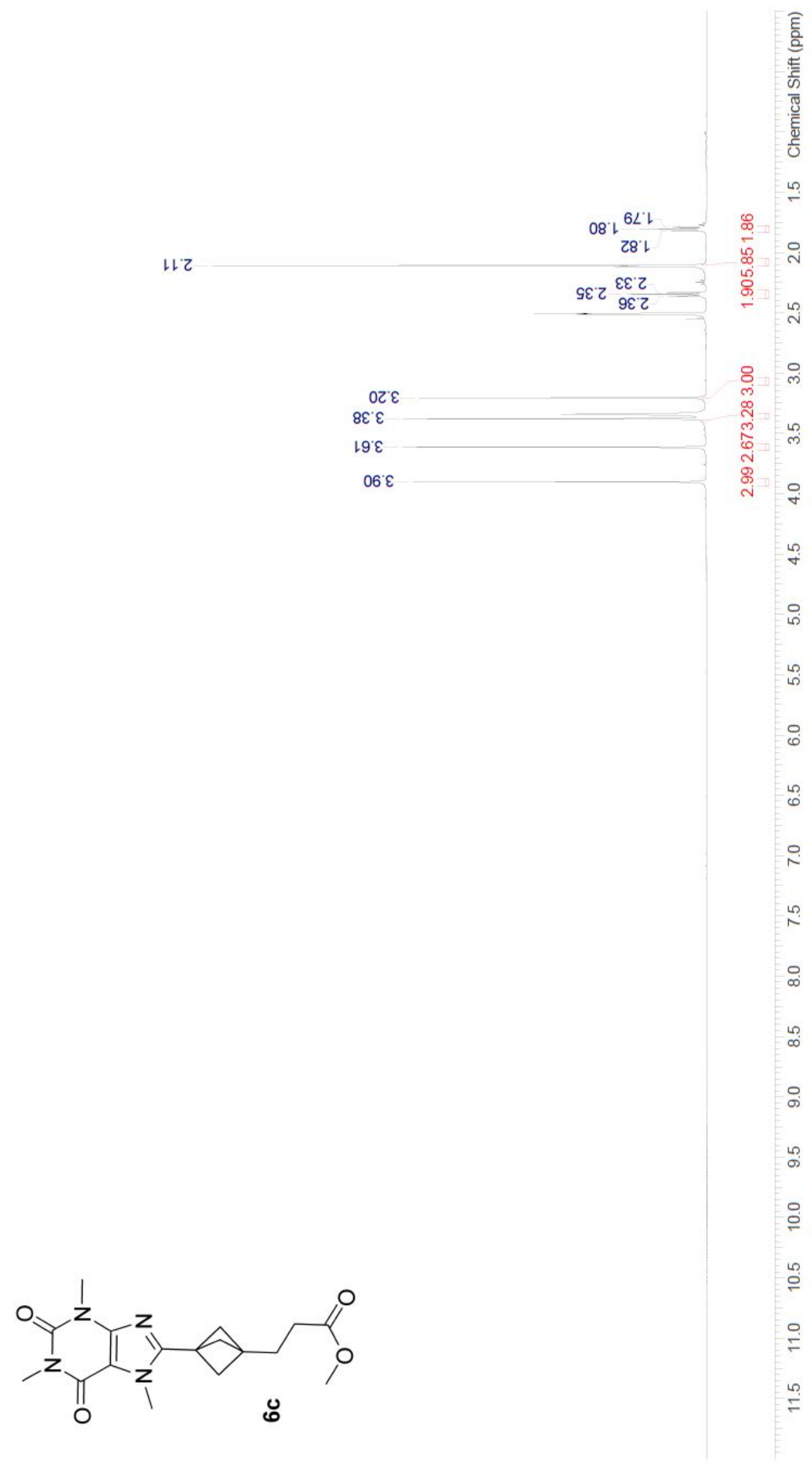




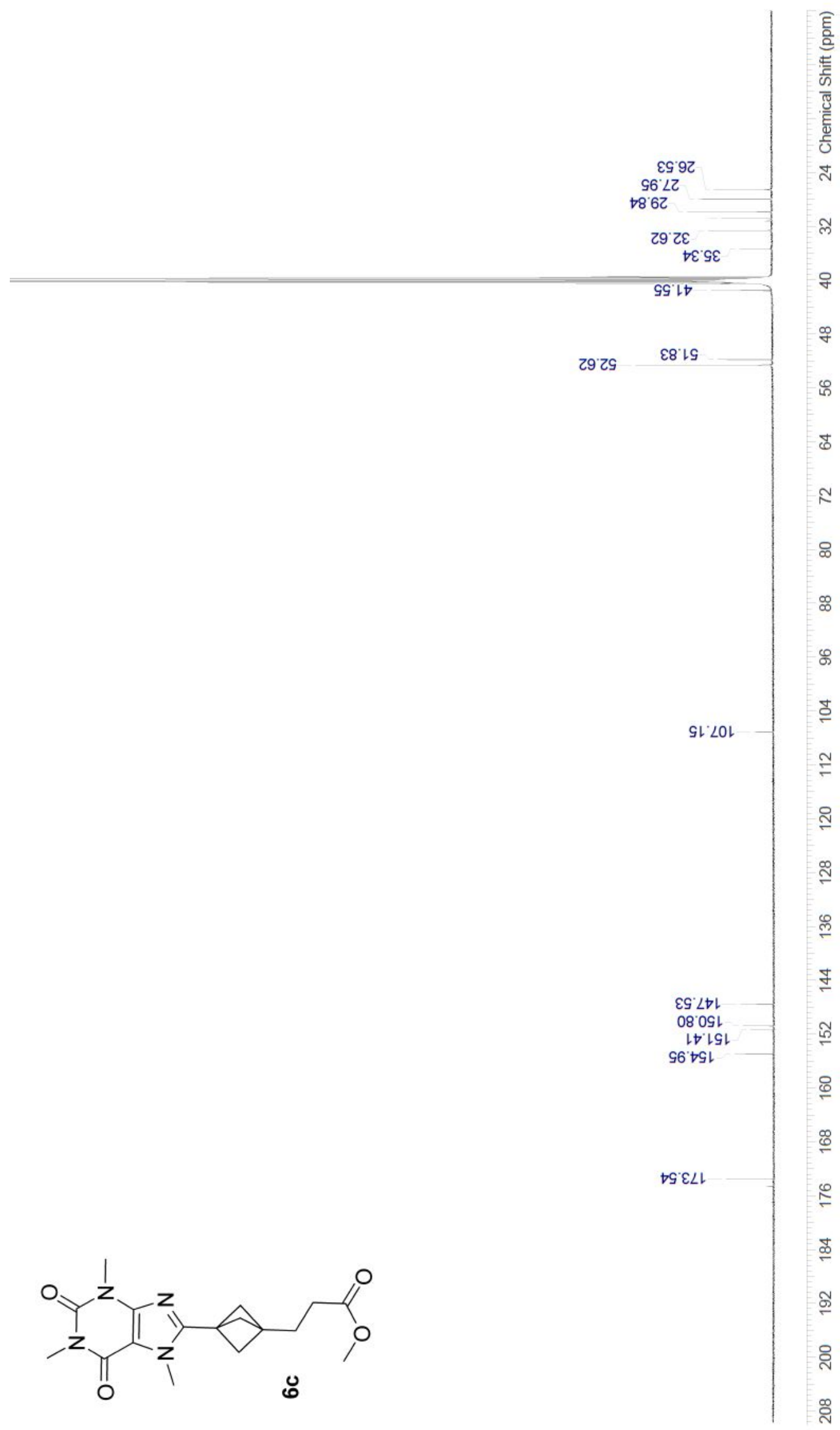




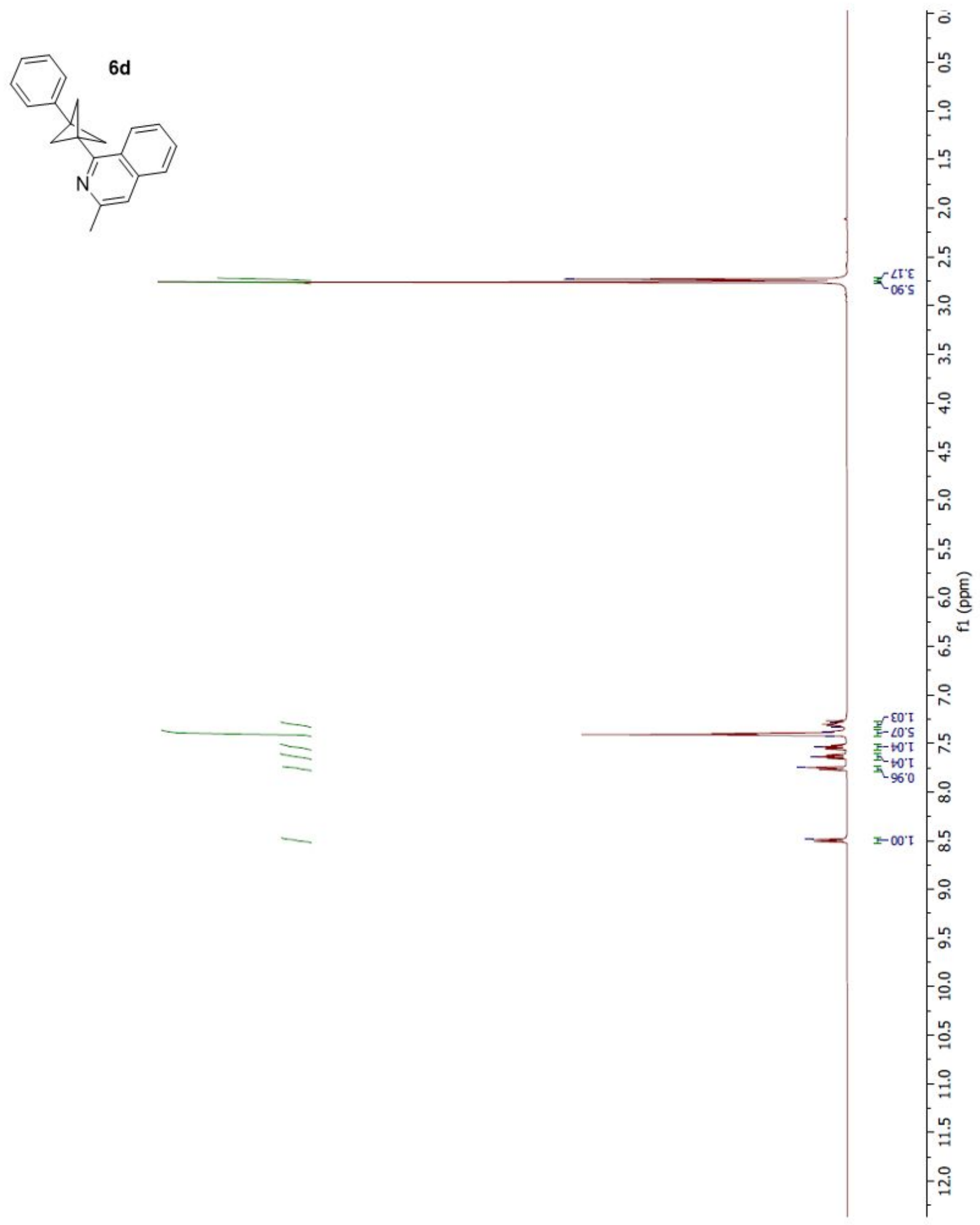




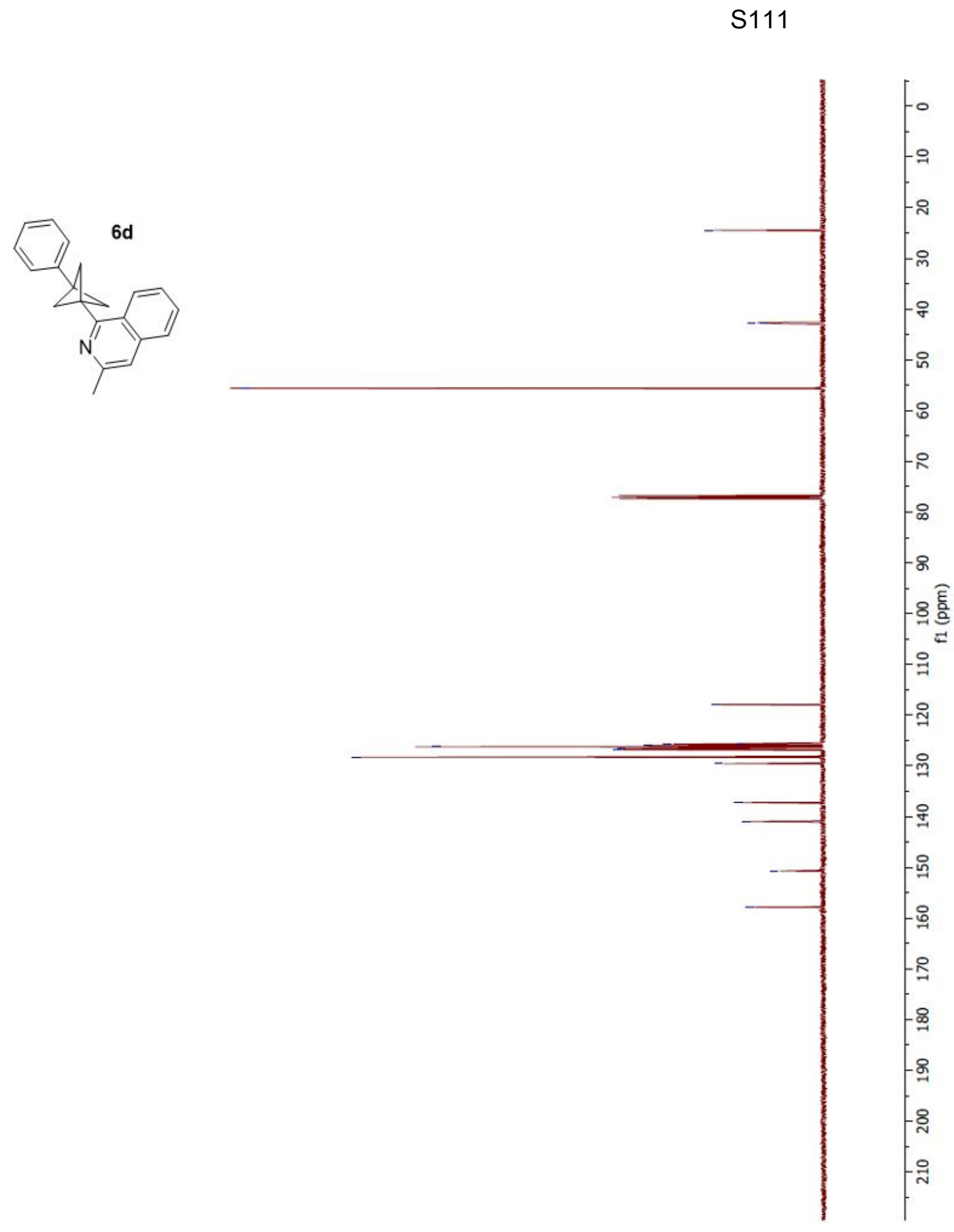




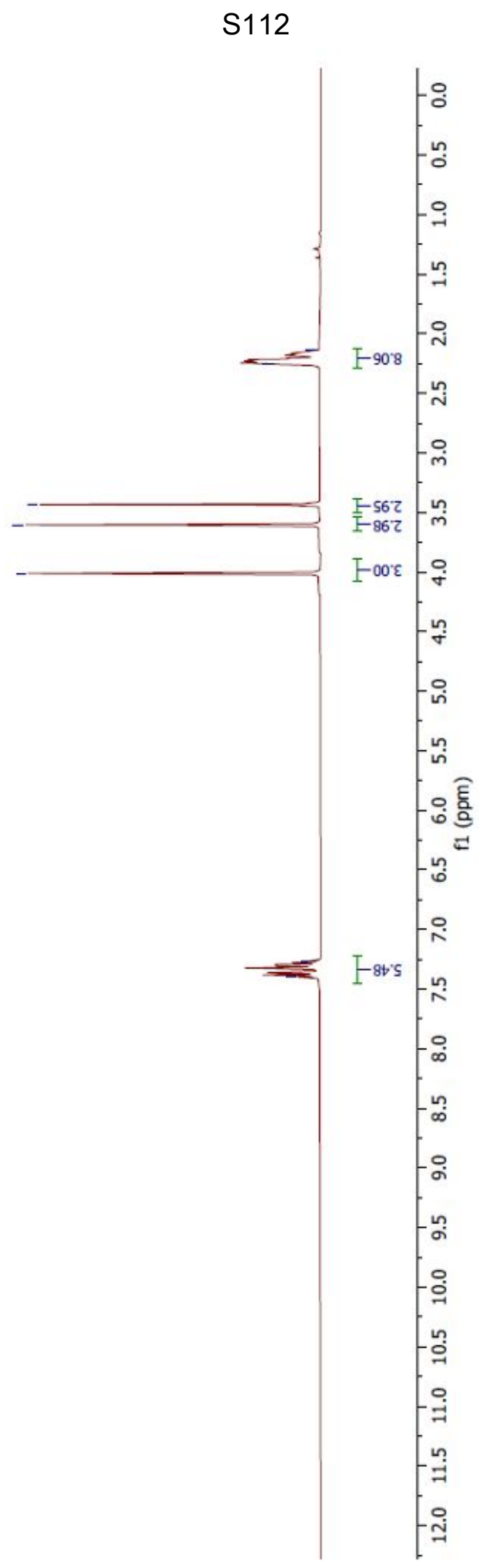




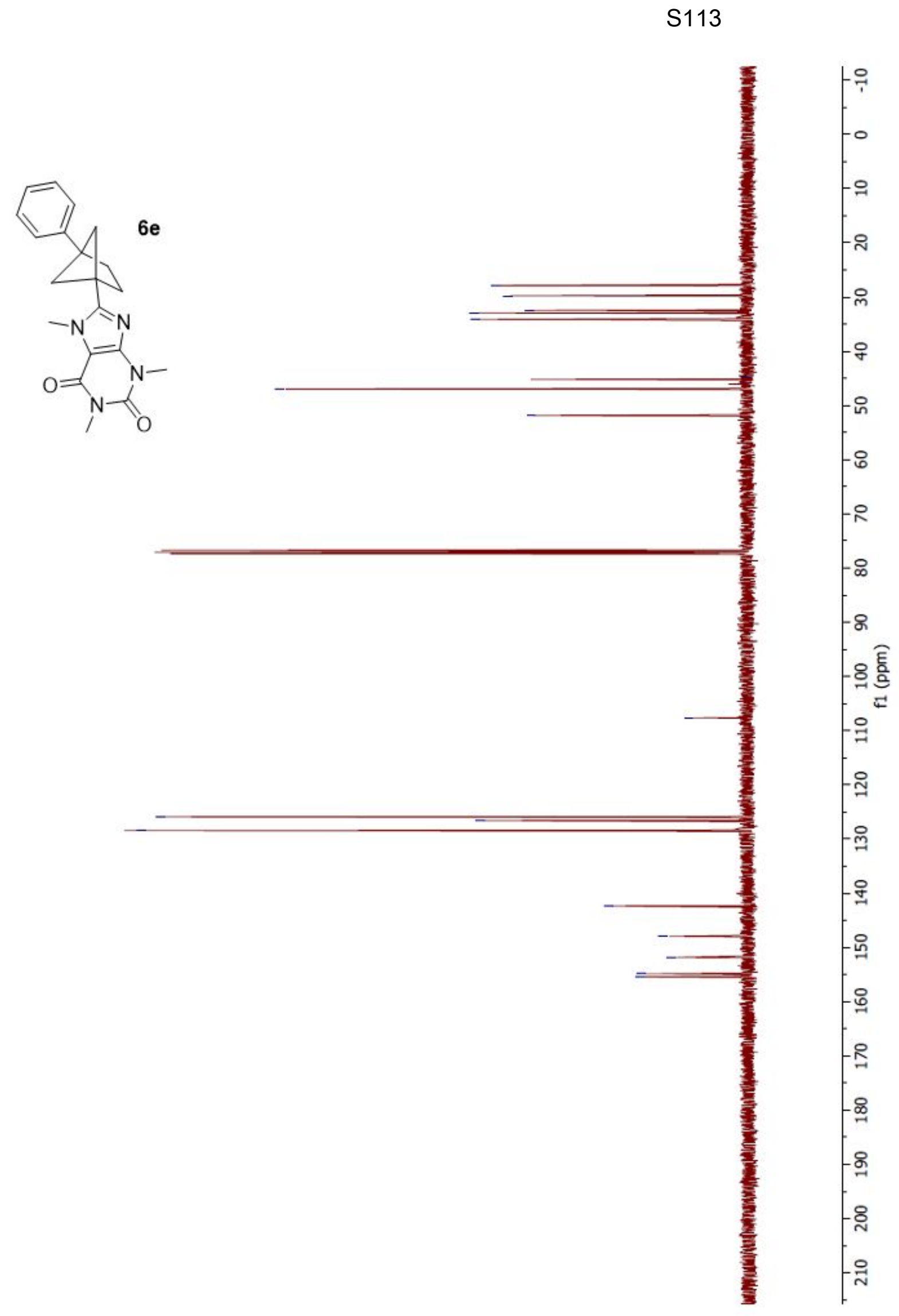




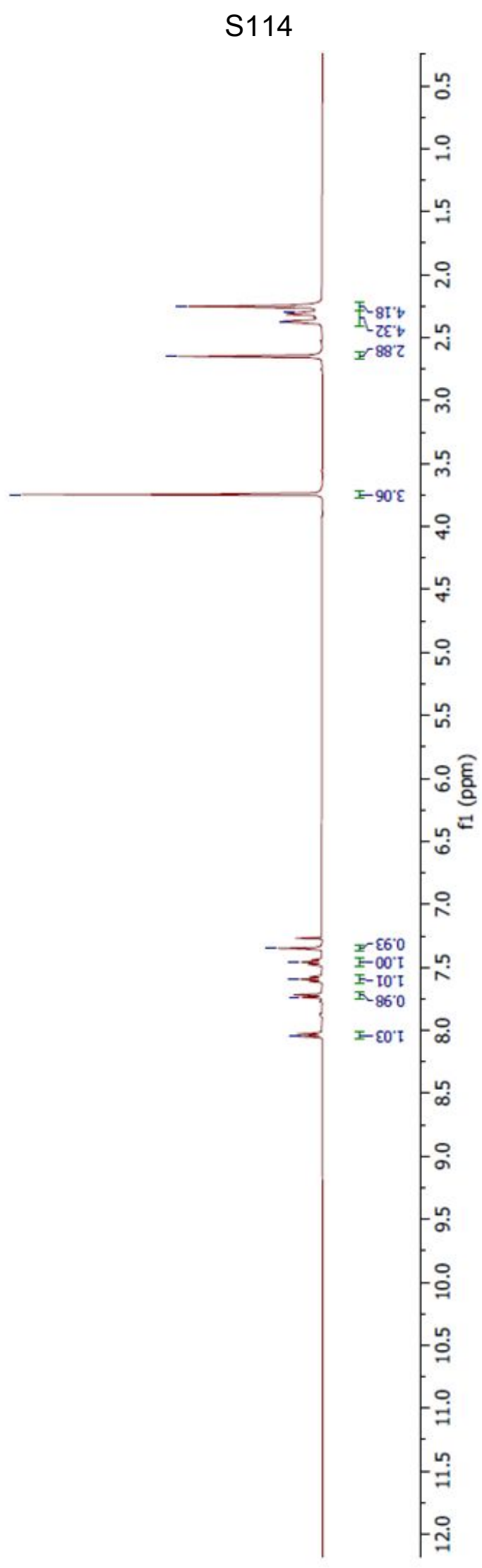




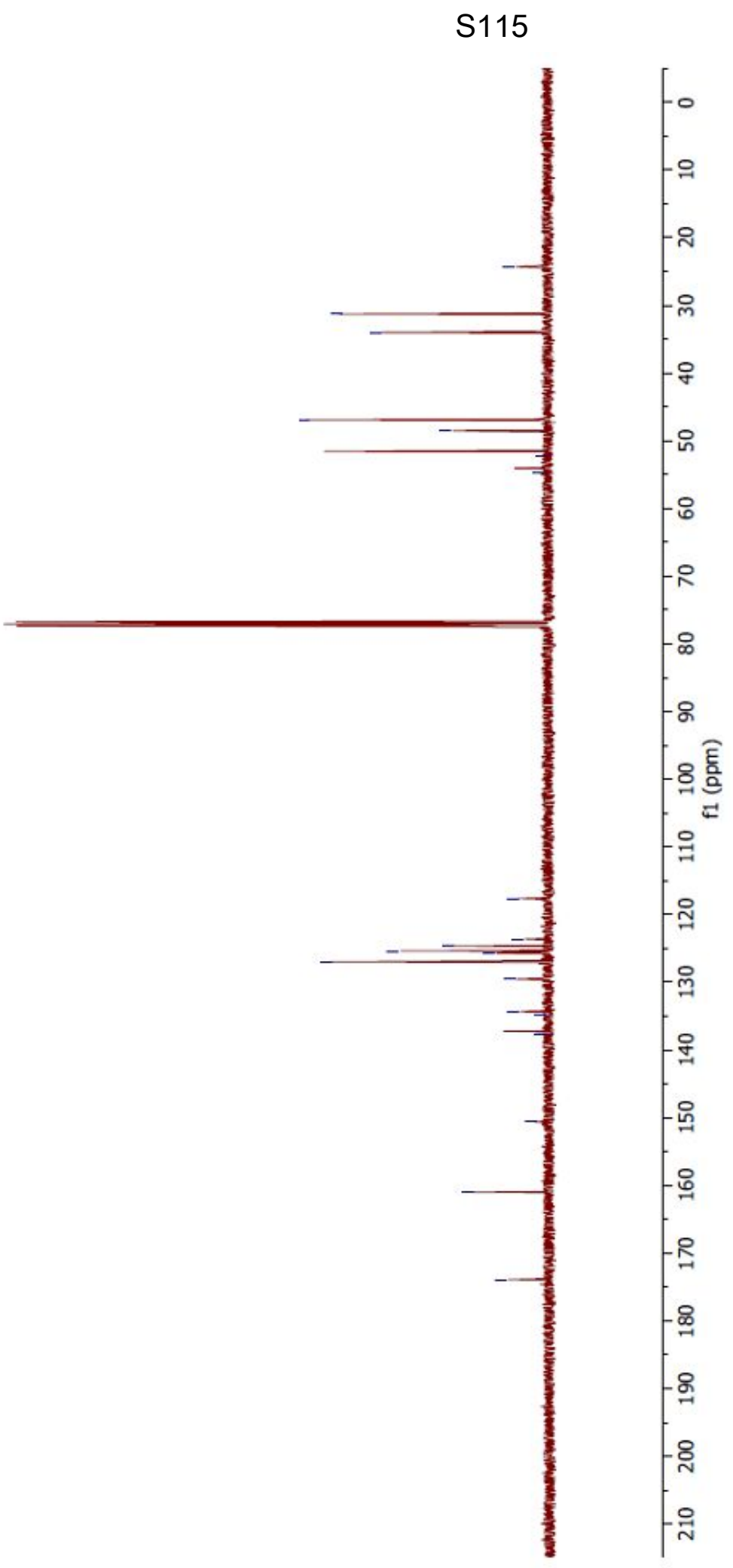




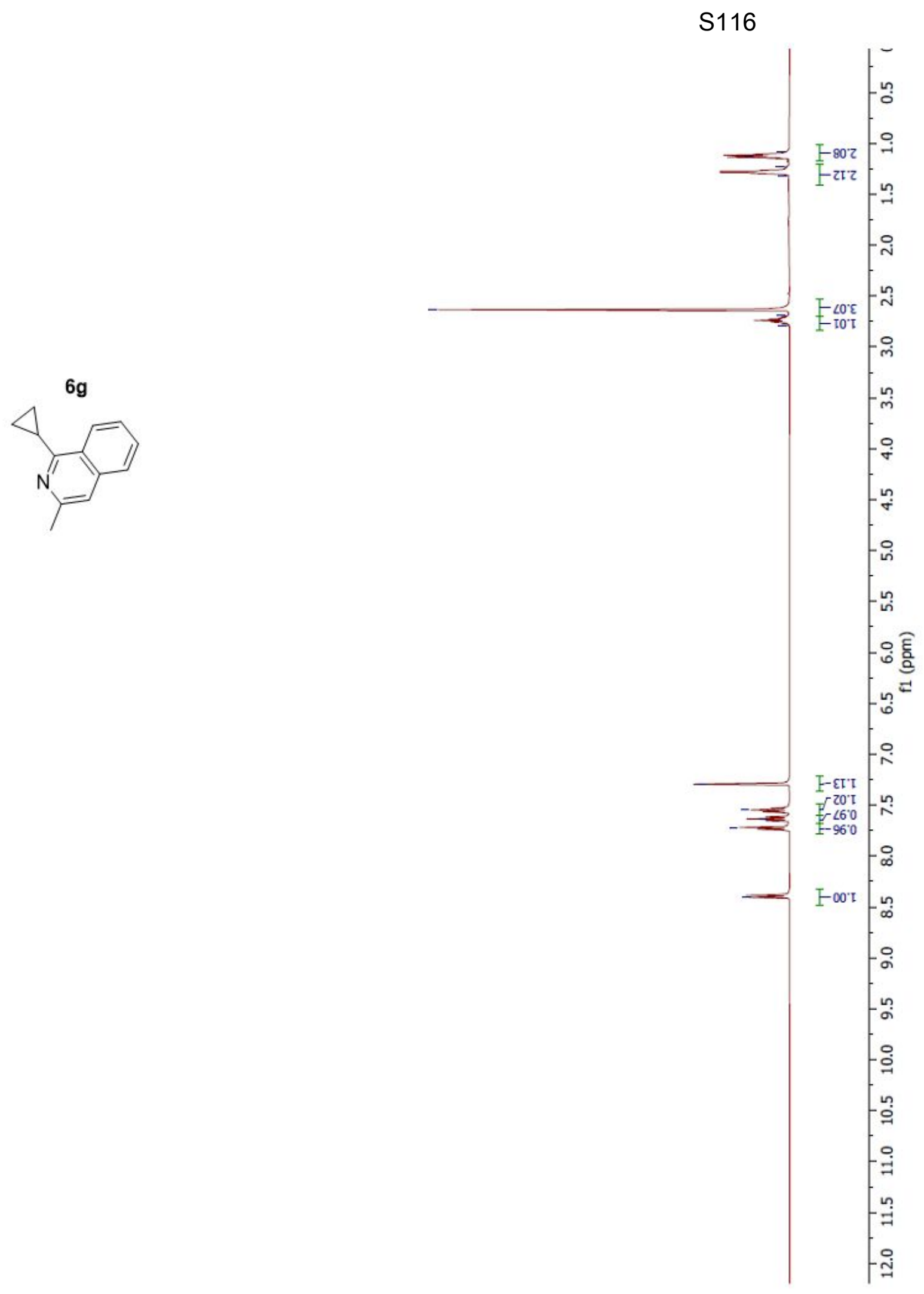




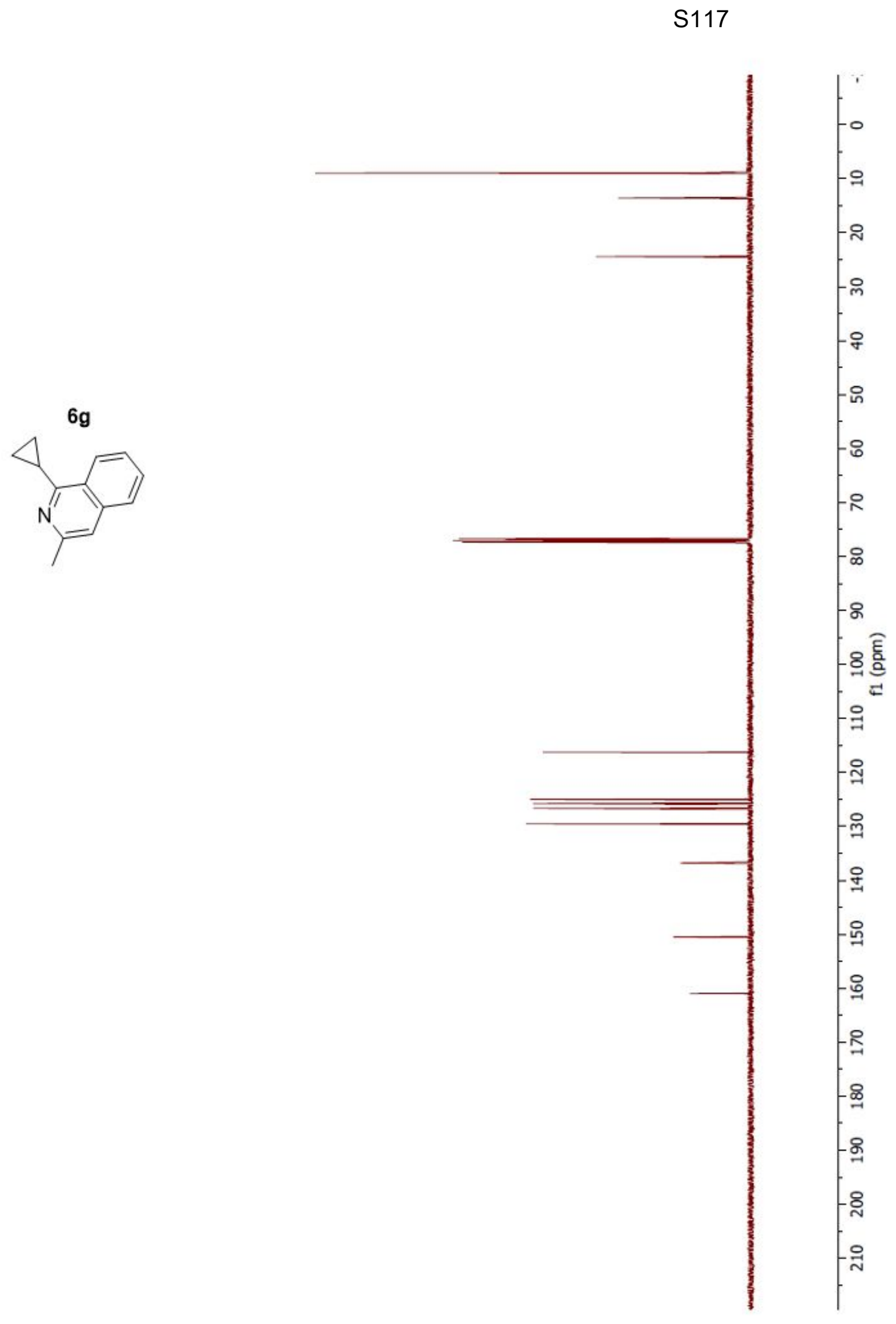


S118

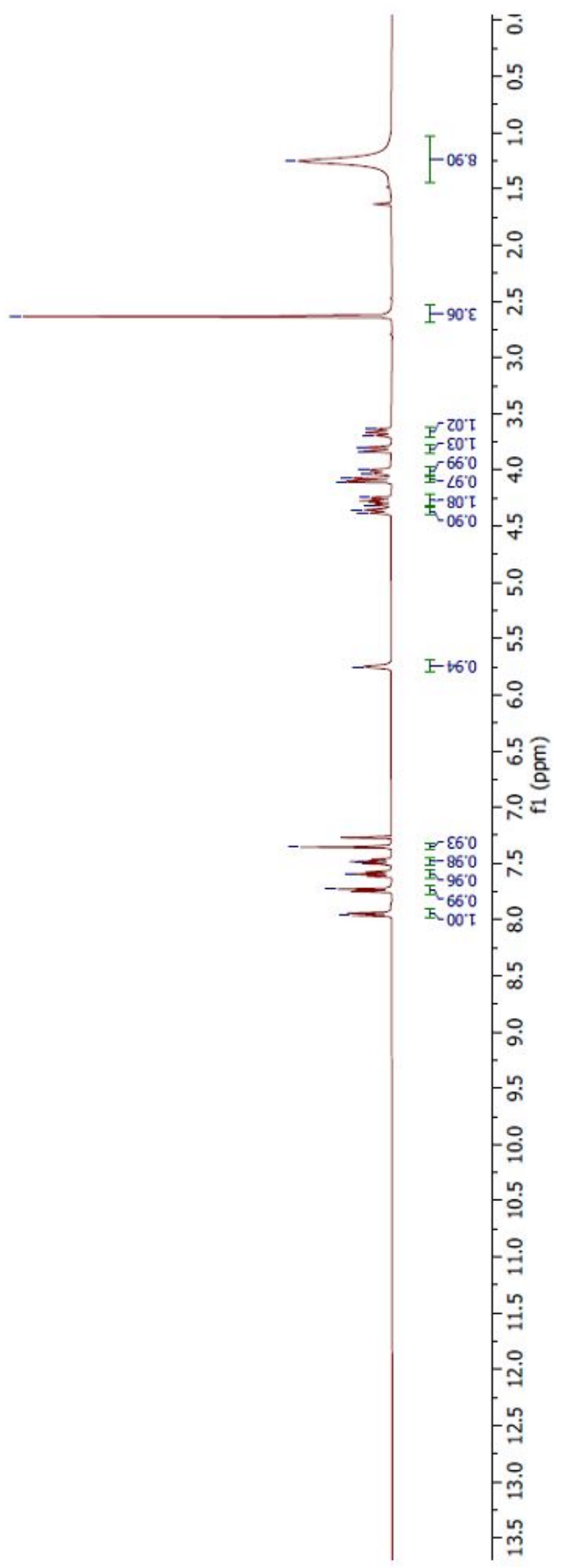




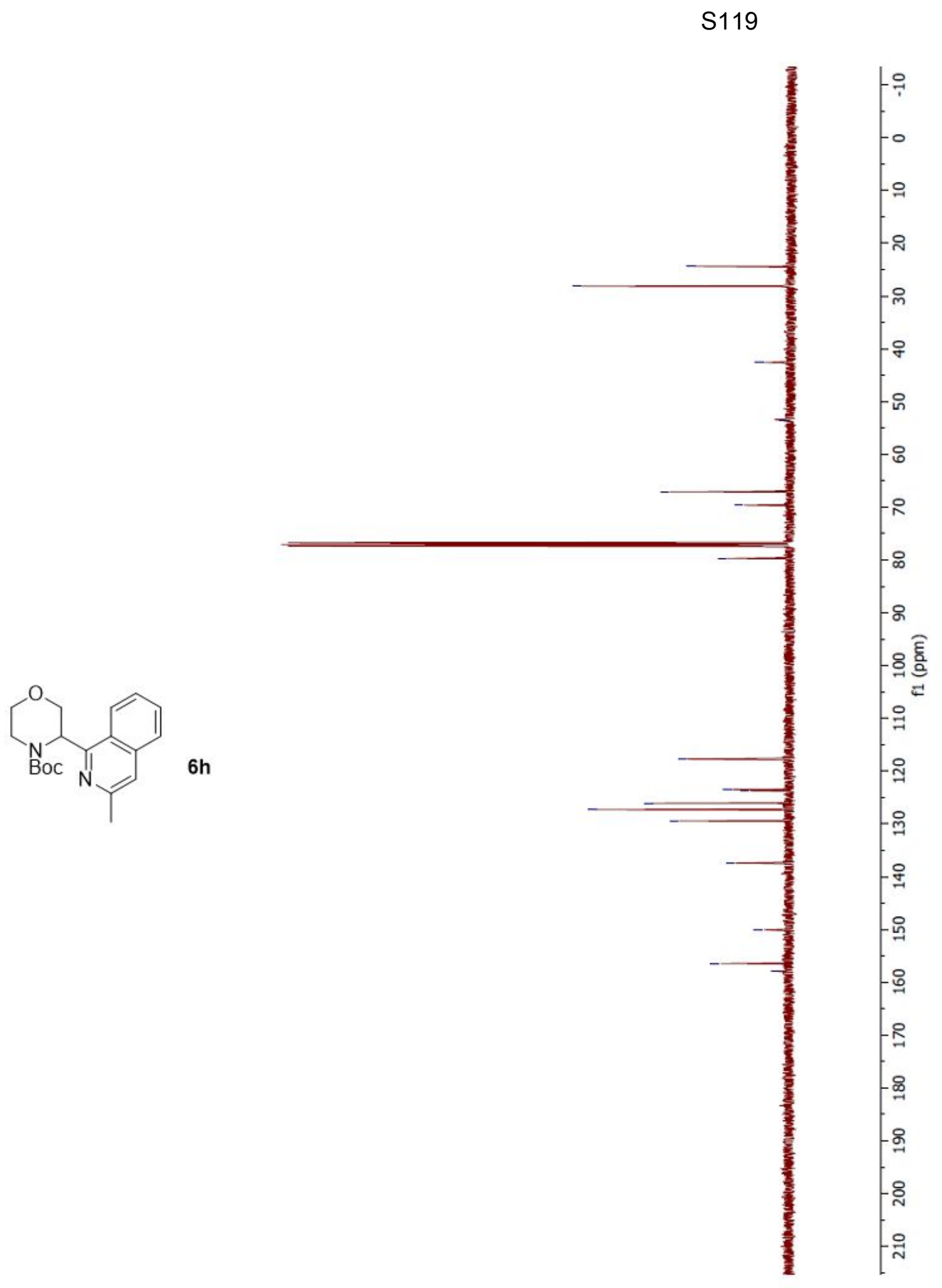




\section{$\underline{\text { References }}$}

(1) Van Heyst, M. D.; Qi, J.; Roecker, A. J.; Hughes, J. M. E.; Chemg, L.; Zhao, Z.; Yin, J. Continuous Flow-Enabled Synthesis of Bench-Stable Bicyclo[1.1.1]pentane Trifluoroborate Salts and Their Utilization in Metallaphotoredox Cross-Coupling Org. Lett. 2020, 22, 1648.

(2) Polites, V. C.; Badir, S. O.; Keess, S.; Jolit, A.; Molander, G. A. Nickel-Catalyzed Decarboxylative Cross-Coupling of Bicyclo[1.1.1]pentyl Radicals Enabled by Electron Donor-Acceptor Complex Photoactivation. Org. Lett. 2021, 23, 4828.

(3) Li, H.; Breen, C. P.; Seo, H.; Jamison, T. F.; Fang, Y-Q.; Bio, M. M. Ni-Catalyzed Electrochemical Decarboxylative C-C Couplings in Batch and Continuous Flow Org. Lett. 2018, 20, 1338.

(4) Fu, M-C; Shang, R.; Zhao, B.; Wang, B.; Fu, Y. Photocatalytic Decarboxylative Alkylations Mediated by Triphenylphosphine and Sodium Azide Science 2019, 6434,1429. 